\title{
Desigualdad de oportunidades educativas en Argentina
}

\author{
Monserrat Serio
}

Director: Mariana Marchionni

Co-director: Leonardo Gasparini

Diciembre 2016

Doctorado en Economía

Universidad Nacional de La Plata 
A la educación suele atribuírsele un rol clave para fomentar la movilidad social ascendente, gracias a su acción igualadora de oportunidades. En general, se la considera el principal medio por el cual los individuos pueden igualarse en oportunidades y revertir la situación social que les ha tocado vivir, para luego ingresar al mercado laboral, generar ingresos y desarrollar su plan de vida. Este escenario ideal, sin embargo, puede no corresponderse con la realidad: a menudo el sistema educativo no cumple una acción igualadora. Es usual que en la etapa de formación educativa se visualicen desigualdades en términos de oportunidades. Como consecuencia de sus entornos, algunos niños y jóvenes, no gozan de las mismas posibilidades educativas que otros.

Mientras más alta sea la desigualdad de oportunidades educativas menor será la movilidad social y más dependientes serán los ingresos de una persona en relación a su entorno. De ahí la relevancia de estudiar y estimar el grado de desigualdad de oportunidades educativas en diferentes países y en el tiempo.

Esta tesis contiene tres capítulos sobre desigualdad de oportunidades educativas en Argentina. Los tres capítulos combinan estrategias empíricas rigurosas con el fin de caracterizar, analizar y proveer mediciones de desigualdad de oportunidades en el país.

El estudio se enfoca en las distribuciones de resultados educativos y los factores que la generan. Siguiendo a Roemer (1998), para que haya igualdad de oportunidades educativas las distribuciones deberían ser independientes de los factores exógenos, tales como el origen social y familiar de los alumnos, que en la literatura se conocen como circunstancias. La desigualdad solo debería surgir como resultado de las diferencias en esfuerzos, elecciones y talentos de las personas.

En el Capítulo 1 se analiza para Argentina la desigualdad de oportunidades en el acceso a educación media, acceso a calidad educativa y desempeño educativo. Se propone un modelo de desigualdad de oportunidades donde se dividen los factores que afectan a la distribución de acceso y desempeño en aceptables (esfuerzos) y no aceptables (circunstancias). Se consideran aceptables la edad del joven y el grado que cursa y no aceptables la educación y ocupación de los padres, la conformación del hogar, el género, el idioma del hogar, el estatus migratorio, los recursos educativos en el hogar y la localización geográfica. Se estiman, en función de estas variables, modelos de probabilidad de acceso a educación y acceso a calidad educativa, así como un modelo de desempeño educativo. Luego, se obtienen distribuciones contrafactuales otorgando a los individuos las mismas características aceptables de tal manera que solo varíen en características no aceptables. Por último, para medir el grado de desigualdad de oportunidades se calculan índices de desigualdad como el coeficiente de Gini, el desvío 
estándar y el índice de disimilitud a partir de las distribuciones contrafactuales obtenidas. Los resultados muestran una baja desigualdad de oportunidades en cuanto al acceso a la educación media y una alta desigualdad de oportunidades para acceder a escuelas que brindan una calidad educativa superior. En el caso de las competencias de los alumnos, los resultados indican una desigualdad de oportunidades significativa, representando más de un tercio de la desigualdad total. Esto sugiere que en el país los jóvenes poseen las mismas oportunidades para acceder a la educación media pero no para acceder a una educación media de calidad o desarrollar sus competencias. De esta manera los alumnos de contextos más desfavorecidos parten con desventajas a la hora de ingresar al mercado laboral o a la educación universitaria.

El Capítulo 2 profundiza el análisis de desigualdad de oportunidades del desempeño educativo. Se examinan los cambios distributivos en las competencias de los alumnos entre 2000 y 2012 y se estudian las principales fuentes de desigualdad. Para ello se realizan microdescomposiciones utilizando un método propuesto por Firpo et al. (2009) que permite realizar descomposiciones de los distintos momentos de las distribuciones. A partir de la descomposición del coeficiente de Gini y las brechas entre cuantiles, se identifican los distintos factores que han contribuido al aumento de la desigualdad de oportunidades en la última década. Los resultados indican que en los últimos años Argentina no ha podido reducir fuertemente la desigualdad de oportunidades educativas. En particular, cuando se analiza en forma detallada la contribución individual de cada factor, se encuentra que los recursos educativos y la localización del colegio contribuyeron a incrementar la desigualdad en estos años.

Finalmente, el Capítulo 3 se enfoca en las diferencias educativas entre escuelas de gestión pública y de gestión privada. Estas diferencias pueden ser una fuente de desigualdad de oportunidades si la calidad educativa es mayor en las escuelas privadas y acceder a las mismas está ligado al nivel socioeconómico familiar. El principal objetivo de este capítulo es aportar nueva evidencia empírica sobre desigualdad de oportunidades entre escuelas de gestión pública y de gestión privada a partir de un análisis no paramétrico para lograr un mayor entendimiento de las desigualdades educativas existentes en el país así como de algunos patrones observados en las últimas décadas como puede ser el incremento de la matrícula de las escuelas de gestión privada. Mientras que en los capítulos anteriores se analiza la desigualdad de oportunidades educativas a partir de medidas resumen de las distribuciones, en este capítulo se analiza la distribución de resultados en forma completa. Los resultados sugieren que los grupos de alumnos que asisten a escuelas de gestión pública y a escuelas de gestión privada notoriamente no enfrentan las mismas oportunidades. Las oportunidades de alumnos de escuelas de gestión pública de contextos favorables solo se igualan con las de aquellos alumnos que asisten a escuelas privadas pero que presentan circunstancias más desfavorables. De alguna manera esto ofrece evidencia de porqué en los últimos años las familias han sustituido las escuelas de gestión pública por las escuelas de gestión privada. Por último, los alumnos que tienen una educación privada y provienen de contextos más favorables son los que presentan las mayores ventajas respecto al resto; mientras que los alumnos de contextos más desfavorables que asisten a la escuela pública son los que menos ventajas tienen. Evidencia de que la escuela pública ha perdido el rol igualador que la sociedad argentina le ha otorgado históricamente. 


\section{Agradecimientos}

Quiero agradecer muy especialmente a mi directora de tesis Mariana Marchionni y codirector Leonardo Gasparini por haberme guiado y alentado a investigar sobre este tema. Estoy inmensamente agradecida por su orientación, apoyo, sugerencias, intercambio de ideas y respaldo durante todo el proceso de tesis. Agradezco a las autoridades del Comité de Doctorado de la Universidad Nacional de La Plata por el aporte de ideas y sugerencias a lo largo de los seminarios del doctorado. A mis compañeros de Doctorado así como a los participantes de los seminarios. Quisiera agradecer al Consejo Nacional de Investigaciones Científicas y Técnicas por el apoyo económico-financiero recibido a través de una beca doctoral y a mi director de beca Ricardo Leiva por sus valiosos consejos. Gracias a todas las sugerencias y comentarios recibidos por distintos colegas en congresos y reuniones científicas donde expuse algunas partes de esta tesis. A María Florencia Gabrielli, Roberto Latorre, Gustavo Maradona y María Inés Lara de la Universidad Nacional de Cuyo por sus comentarios sumamente valiosos. A Andrés por su paciencia e inmenso apoyo en todo este proceso. Finalmente, un especial agradecimiento a mi familia Virginia, Daniel, Facundo y Ulises, que siempre he contado con el apoyo incondicional de cada uno. 


\section{Índice general}

$\begin{array}{ll}\text { Introducción } & 1\end{array}$

Capítulo 1

1. Desigualdad de oportunidades educativas en Argentina desde tres di$\begin{array}{ll}\text { mensiones: acceso, calidad y desempeño educativo. } & 6\end{array}$

1.1. Introducción . . . . . . . . . . . . . . . . . . . . 6

1.2. Metodología . . . . . . . . . . . . . . . . . 8

1.2.1. Desigualdad de oportunidades educativas . . . . . . . . . . . . . 8

1.2.2. Conjunto de atributos . . . . . . . . . . . . . . . . . . . . . . . . . . . . . . . . 10

1.2.3. Estrategia empírica . . . . . . . . . . . . . . . . 12

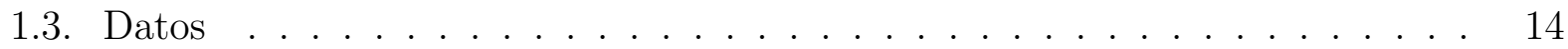

1.4. Resultados . . . . . . . . . . . . . . . . . . . 18

1.4.1. Acceso a educación media . . . . . . . . . . . . . . . . . . 19

1.4.2. Acceso a calidad educativa . . . . . . . . . . . . . . . . . 21

1.4.3. Desempeño educativo . . . . . . . . . . . . . . . 25

1.5. Conclusiones . . . . . . . . . . . . . . . . . . . . . . . . . . . . 28

\section{Capítulo 2}

2. Cambios distributivos en el desempeño educativo. Evidencia de desigualdad de oportunidades a partir de un enfoque de regresiones de funciones de influencia recentradas.

2.1. Introducción . . . . . . . . . . . . . . . . . . . . . . . . . 31

2.2. Metodología . . . . . . . . . . . . . . . . . 33

2.2.1. Método de descomposición a partir de regresiones de RIF . . . . . . . 33

2.2.2. Medida de desigualdad de oportunidades . . . . . . . . . . . . . 37

2.2.3. Estimación . . . . . . . . . . . . . . . . . . . . . . . . . . . . . . . . 37

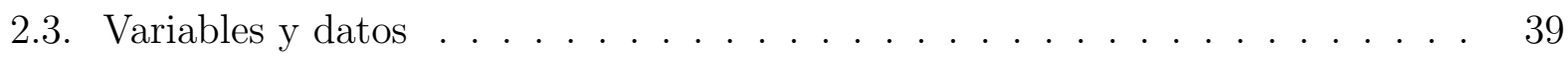

2.4. Resultados . . . . . . . . . . . . . . . . . . . . 43

2.4.1. Descomposición de desigualdad de oportunidades educativas más allá de la media. . . . . . . . . . . . . . . . . . . . . 43

2.4.2. Extensiones . . . . . . . . . . . . . . . . 53

2.5. Conclusiones . . . . . . . . . . . . . . . . . . . 55

\section{Capítulo 3}

3. El papel de las escuelas públicas y privadas en la igualdad de oportunidades en Argentina. Un análisis de dominancia estocástica.

3.1. Introducción . . . . . . . . . . . . . . . . . . . . . 58

3.2. Metodología . . . . . . . . . . . . . . . . . . . . . . 59 
3.2.1. Marco teórico . . . . . . . . . . . . . . . . . . . . . 60

3.2.2. Condiciones necesarias y suficientes para la existencia de IOP . . . . 62

3.2.3. Test de igualdad de oportunidades . . . . . . . . . . . . . . . . 65

3.3. Definición de variables y datos . . . . . . . . . . . . . . . . . . . . . . . . . . . . . . .

3.4. Resultados . . . . . . . . . . . . . . . . . . 72

3.5. Conclusiones . . . . . . . . . . . . . . . . . . . 85

$\begin{array}{lr}\text { Comentarios finales } & 88\end{array}$

$\begin{array}{lr}\text { Bibliografía } & 90\end{array}$

$\begin{array}{lr}\text { Anexos } & 96\end{array}$

A1.Capítulo $1 \ldots \ldots \ldots \ldots \ldots \ldots$

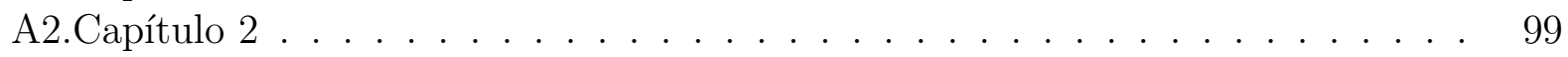

$\begin{array}{ll}\text { Apéndices } & 102\end{array}$

A1.Capítulo $1 \ldots \ldots \ldots \ldots \ldots$. . . . . . . . . . . . . . . . . . . . . . . . . . . . . . . . . . .

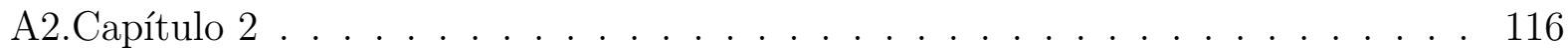

A3.Capítulo $3 \ldots \ldots \ldots \ldots \ldots \ldots \ldots \ldots$ 



\section{Introducción}

En la actualidad, la concepción más aceptada y extendida de equidad económica se corresponde con la idea de "igualdad de oportunidades" (Atkinson y Bourguignon, 2015). Si bien la mayoría de las personas coinciden en su importancia, llegar a un acuerdo sobre qué significa "las mismas oportunidades" no es una tarea sencilla. La igualdad de oportunidades es un concepto estrechamente relacionado con la equidad y, por lo tanto, su definición no está exenta de una gama de posiciones normativas. En este estudio se entiende por igualdad de oportunidades a una situación donde todos los individuos disponen de las mismas posibilidades para lograr distintos resultados más allá del contexto y desventajas que enfrenten en su vida, independientemente de si alcancen o no el resultado final, y se la asocia a la noción de "nivelar el campo de juego" de Roemer (1998).

La idea de igualar oportunidades ha tomado cada vez mayor relevancia en los últimos años, estimulando su estudio científico desde una perspectiva económica. Influyentes académicos, tanto filósofos como economistas (Dworkin, 1981; Arnerson, 1989; Cohen, 1989; Roemer, 1998; Sen, 2000, entre otros), han argumentado que la igualdad de resultados no es la más apropiada para establecer si un sistema social es justo, ya que algunas brechas de resultados pueden ser aceptables para la sociedad. En este contexto surge la igualdad de oportunidades como un nuevo enfoque distributivo.

La igualdad de oportunidades no garantiza la igualdad de resultados. Es posible que en un contexto de igualdad de oportunidades, alguna desigualdad pueda ser socialmente aceptable si proviene de los esfuerzos y talentos de las personas. Si bien este enfoque establece nuevos desafíos teóricos y metodológicos, avanzar en esta nueva línea resulta relevante para el desarrollo de nuevos criterios distributivos. Un mayor entendimiento de la igualdad de oportunidades en la desigualdad de resultados puede tener profundas consecuencias sobre la percepción de la equidad en una sociedad y, por lo tanto, sobre las políticas redistributivas. A continuación se presenta una breve revisión de la literatura.

Roemer (1998), uno de los principales exponentes de la teoría de igualdad de oportunidades, propone analizar la distribución de resultados de los individuos a partir de los factores que generan dicha distribución. Divide los factores entre esfuerzos y circunstancias. Los esfuerzos son variables que pueden ser controlables por los individuos y se considera que los individuos son responsables de los resultados generados como consecuencia de esos esfuerzos. En cambio, las circunstancias, como el origen social y familiar, son exógenas a los individuos y, por lo tanto, ellos no son completamente responsables de los resultados producidos por éstas. La desigualdad de resultados generada por las circunstancias se considera injusta y es la que habría que corregir. Así Roemer (1998) define como igualdad de oportunidades a una 
situación donde los resultados de los individuos son distribuidos independientemente de las circunstancias.

En los últimos años se ha desarrollado una literatura tendiente a obtener mediciones del grado de desigualdad de oportunidades. Como primer paso en esta dirección varios autores se han enfocado en la medición de la desigualdad de oportunidades de ingresos o de consumo (Peragine, 2004; Checchi y Peragine, 2005, 2010; Checchi et al., 2008; Björklund et al., 2012; Bourguignon et al., 2007a, 2013, 2007b; Lefranc et al., 2008, 2009; Paes et al., 2008; Ferreira y Gignoux, 2011; Ferreira et al., 2011; Singh, 2012; Yalonetzky, 2012; Ferreira y Gignoux, 2014; Brunori et al., 2013; Milanovic, 2015; Kanbur y Stiglitz, 2016). Metodológicamente, algunos autores como Checchi y Peragine (2010) estudian la desigualdad de oportunidades de ingresos en Italia a partir de un enfoque no paramétrico. Siguiendo a Roemer (1998) dividen a la población en "types" (grupos de personas que presentan las mismas circunstancias) y utilizando técnicas de microdescomposición analizan la desigualdad entre grupos, la que interpretan como desigualdad de oportunidades. También agrupan personas que han ejercido el mismo nivel de esfuerzo y llaman a estos grupos "tranches" (tramos). La desigualdad de oportunidades en este último caso, viene dada por la desigualdad dentro de los grupos. Por otro lado, Lefranc et al. (2008) trabajan con tests estadísticos de dominancia estocástica de primer orden y de segundo orden de funciones de distribución condicionadas en "types", con el fin de encontrar evidencia de desigualdad de oportunidades de ingreso para distintos países de Europa y Estados Unidos.

Un enfoque paramétrico, es el propuesto por Bourguignon et al. (2007a, 2013), que consiste en estimar una ecuación de resultados (ingresos, salarios y consumo) en función de variables de circunstancias y de esfuerzo, y simular distribuciones de resultados contrafactuales que incluyan solo los efectos de las variables de circunstancias.

Respecto a educación, si bien existe abundante literatura sobre la función de producción educativa y sobre desigualdades educativas y su relación con los ingresos laborales, es menos copiosa la literatura que analiza desigualdad de oportunidades en educación. Algunos de ellos son Roemer (1998), Gasparini (2002), Betts y Roemer (2006), Brunello y Checchi (2007), Waltenberg y Vandenberghe (2007), Schütz et al. (2008), Paes et al. (2008), Gamboa y Waltenberg (2012), Ferreira y Gignoux (2014), Salehi-Isfahani et al. (2014), Gamboa y Waltenberg (2015).

Paes et al. (2008) analizan la desigualdad de oportunidades en el acceso a educación para cinco países de América Latina (Argentina, Brasil, Chile, México y Perú). Para ello proponen un Índice de Oportunidades Humanas (HOI, por sus siglas en inglés), basado en el índice de disimilitud de la teoría de segregación y en las probabilidades de acceso (estimadas a partir de modelos no lineales con métodos paramétricos). ${ }^{1}$

Gasparini (2002) estudia la desigualdad de oportunidades en el acceso a la educación media en Argentina causada por diferencias en variables socialmente inaceptables a partir de métodos no paramétricos. Encuentra que la tasa de asistencia del nivel secundario está estrechamente relacionada a este tipo de variables, lo que daría cuenta de algún grado de desigualdad de oportunidades en el acceso.

Por otro lado, algunos autores se han enfocado en la desigualdad de oportunidades en el desempeño educativo, utilizando como proxy de logros educativos los puntajes de los exámenes de pruebas estándares, como las pruebas internacionales del Programa para la Evaluación Internacional de Alumnos (PISA) de la Organización para la Cooperación y el Desarrollo Económicos (OCDE) o el Estudio de las Tendencias en Matemáticas y Ciencia (TIMSS) de la Asociación Internacional para la Evaluación del Rendimiento Educativo (IEA,

\footnotetext{
${ }^{1}$ También incluyen un análisis de la desigualdad de oportunidades en el acceso a servicios de agua, cloacas y electricidad.
} 
por sus siglas en inglés). Schütz et al. (2008) proporcionan una medida de desigualdad de oportunidades educativas en 54 países, estimando el efecto de los antecedentes familiares en el rendimiento de los estudiantes en dos pruebas internacionales TIMSS. Por su parte, Ferreira y Gignoux (2014) miden la desigualdad de oportunidades en los logros educativos utilizando los puntajes de las pruebas PISA de 2006 en 57 países, y encuentran que alrededor del $10 \%$ y $30 \%$ de la distribución de los puntajes puede interpretarse como desigualdad de oportunidades.

Esta tesis se enmarca dentro de esta última literatura. Siguiendo la evidencia empírica reciente, la mayoría de los estudios se han enfocado en la distribución de ingresos, esta tesis lo hace en la dimensión educativa. Se aborda esta dimensión porque se considera que la educación es el primer paso antes de ingresar al mercado laboral: ${ }^{2}$ la educación puede brindar a los individuos las oportunidades para igualar el "campo de juego". Se trabaja principalmente con la teoría propuesta por Roemer (1998) y se realiza un análisis empírico riguroso que tiene como fin caracterizar la desigualdad de oportunidades educativas en Argentina brindando mediciones e indicadores sobre el grado y la existencia de la misma.

Según la legislación argentina los fines y los objetivos de la política educativa del país deben estar basados en la igualdad de oportunidades. Además, entre sus disposiciones, se establece que se deben garantizar las condiciones para que todos los alumnos del país logren una educación de calidad independientemente de su origen social, radicación geográfica, género o identidad cultural (art. 11 y 84 de la Ley Nacional de Educación Nro. 26.206). A pesar de estar plasmado en la legislación, y en los últimos años haberse incrementado los recursos públicos destinados al sector educativo y haberse financiado diversos programas tendientes a garantizar dichas condiciones como la Asignación Universal por Hijo para la protección social (AUH), el programa Conectar Igualdad, el programa Más Escuelas y la gran variedad de reformas educativas y campañas; cada vez más se encuentra que al egresar del nivel medio los jóvenes no cuentan con las mismas oportunidades para insertarse al mercado laboral o continuar con sus estudios superiores. ${ }^{3}$ Estas brechas educativas atentan contra el rol igualador de la educación y no permiten igualar el campo de juego creando desigualdad de oportunidades. Dado esto, resultan de vital interés las mediciones de desigualdad de oportunidades educativas en el país. Asimismo, medir esta desigualdad resulta clave para diseñar o re-diseñar políticas educativas en pos de aumentar las oportunidades básicas de los grupos más desfavorecidos. Sin embargo, a pesar de que la desigualdad de oportunidades educativas no solo supone un costo notable para el individuo sino también para la sociedad, ya que altera la cohesión y la justicia social, no existen mediciones sistemáticas de la misma. Este estudio tiene por objetivo avanzar sobre esta dirección y brindar evidencia empírica que contribuya a la caracterización de dicha desigualdad en el país.

En el Capítulo 1 se mide la desigualdad de oportunidades en el acceso a educación, en el acceso a calidad educativa y en las competencias de los alumnos del nivel medio en Argentina. Se presenta el marco teórico y se discute la definición de desigualdad de oportunidades aplicada. A partir de este marco, se propone un modelo para cada una de las dimensiones educativas. Se dividen los factores de los alumnos que pueden afectar el resultado educativo

\footnotetext{
${ }^{2}$ Existe una larga literatura que analiza no solo la correlación entre la educación y los salarios, sino también el efecto causal de la misma sobre los ingresos laborales (Card, 1999; Ashenfelter et al., 1999).

${ }^{3}$ En el Ministerio de Educación del país se pueden encontrar más de 25 planes o programas educativos como por ejemplo el programa Nacional de Alfabetización, el plan Nacional FinEs -Finalización de Estudios Primarios y Secundarios-, las Campañas Nacionales de Lectura de los años 2003 a 2007). Además si se ajusta a precios constantes de 1993 utilizando un promedio del índice de precios implícitos del PBI y el índice de precios mayorista se encuentra que tanto el gasto público educativo nacional como provincial han aumentado fuertemente entre 2003 y 2012 (los montos de dichos gastos se cuadruplicaron en dicho periodo). También se encuentra que el gasto educativo se duplicó entre el 2001 (antes de la crisis) y 2012.
} 
en dos grupos: factores socialmente aceptables y no aceptables. La idea detrás es que la probabilidad de éxito educativo de cada alumno, independientemente a que lo alcancen, no debería estar relacionada con factores no aceptables como el origen social o el género. La desigualdad que proviene de estos factores atenta contra la acción igualadora de la educación y no permite nivelar el campo de juego entre los jóvenes, por lo que se puede asociar con desigualdad de oportunidades educativas. Una vez definido el grupo de factores aceptables y no aceptables, se estiman distribuciones contrafactuales de los resultados educativos. Estas distribuciones contrafactuales se obtienen asignando a todos los alumnos las mismas características aceptables y dejando que solo varíen en características no aceptables. Luego, se computa la desigualdad proveniente de cada distribución contrafactual, obteniendo así medidas resumen de desigualdad de oportunidades educativas. Los resultados sugieren que la desigualdad de oportunidades en el acceso a educación media es baja en el país mientras que la desigualdad de oportunidades en el acceso a calidad educativa y desempeño educativo es alta. En particular, se hallan escasas oportunidades para acceder a escuelas que ofrecen una calidad educativa integral. De estos resultados se infiere que uno de los principales desafíos del país, en pos de las oportunidades educativas, es garantizar el acceso a calidad educativa y, de esta manera, disminuir las disparidades en relación a este resultado.

El Capítulo 2 analiza las principales fuentes de desigualdad de oportunidades del desempeño educativo en Argentina a partir de microdescomposiciones. En este capítulo se propone una nueva medida de desigualdad de oportunidades que permite analizar cómo ésta ha cambiado en distintos periodos de tiempo. Como medida del desempeño educativo se consideran los puntajes en matemáticas de las pruebas PISA y se proponen tres modelos de desempeño que incluyen distintas variables aceptables y no aceptables. Los resultados indican que la disminución de la desigualdad encontrada en los 2000s se puede asociar principalmente a factores aceptables y en menor medida a factores no aceptables. Lo que indicaría que en los últimos años Argentina no ha podido reducir fuertemente la desigualdad de oportunidades educativas. A su vez cuando se analiza en forma detallada la contribución individual de cada factor, se encuentra que los recursos educativos y la localización del colegio contribuyeron a incrementar la desigualdad en estos años.

Finalmente, en Argentina los alumnos que asisten a colegios privados presentan, en promedio, un mayor desempeño en las pruebas PISA que aquellos que van a colegios públicos. Estas pruebas evalúan competencias de los alumnos, es decir, si los mismos tienen las capacidades necesarias para poder desarrollarse en una sociedad; lo que indicaría que los alumnos de escuelas públicas no disponen de las mismas capacidades para desarrollarse plenamente en un futuro que alumnos que asisten a escuelas privadas. Por lo tanto, resulta interesante preguntarse si los alumnos de escuelas públicas o privadas cuentan con las mismas ventajas luego de controlar por características individuales o si existen diferencias arraigadas en el sistema educativo argentino que se relacionan con los tipos de establecimiento educativos. El Capítulo 3 se concentra en las oportunidades de los alumnos que asisten a escuelas de gestión pública y privada. Se ordenan las distribuciones de desempeño educativo entre los distintos grupos de alumnos utilizando principios de dominancia estocástica. Los resultados sugieren que los alumnos que asisten a escuelas de gestión estatal son los que más desventajas exhiben respecto a los demás tipos de alumnos, aún controlando por distintas variables de esfuerzo y circunstancias. También se encuentra que los grupos de alumnos más favorecidos por circunstancias externas que asisten a escuelas públicas presentan similares oportunidades que alumnos de escuelas privadas pero de contextos más desfavorecidos, evidencia de porqué las familias en los últimos años se han volcado en enviar a sus hijos a escuelas privadas. En casi todos los casos se encuentra una clara preferencia por las distribuciones de alumnos de escuelas privadas. Por último, se presentan los comentarios finales. 


\section{CAPÍTULO 1}

\section{Desigualdad de oportunidades educativas en Argentina desde tres dimensiones: acceso, calidad y desempeño educativo.}

\subsection{Introducción}

La educación es fundamental para el desarrollo y calidad de vida de las personas. La misma cumple una función de integración y cohesión social y se le suele atribuir un rol clave para la movilidad social ascendente debido a su acción igualadora de oportunidades. Esta concepción de la educación como herramienta para "nivelar el campo de juego" entre individuos de distintos orígenes socioeconómicos se plasma muchas veces en la legislación. Por ejemplo, según la Ley de Educación Nacional Nro. 26.206 (2006) de Argentina uno de los objetivos de la política educativa del país es

"Asegurar una educación de calidad con igualdad de oportunidades y posibilidades, sin desequilibrios regionales ni inequidades sociales." (artículo 11, inciso a).

Si bien la universalidad y la gratuidad de la educación buscan reducir al máximo los desequilibrios y desigualdades que pudieran existir, es posible que subsistan factores que afecten el acceso a la educación, la permanencia en la escuela u obtener un buen rendimiento. Estos factores que atentan contra la acción igualadora, pueden dar lugar a desigualdades de oportunidades educativas.

Este capítulo contribuye al estudio de la desigualdad de oportunidades educativas de los jóvenes en Argentina. El objetivo es cuantificar el grado de esta desigualdad para distintos resultados educativos. Algunas de las preguntas que se pretende responder son: ¿Qué tan desiguales son las oportunidades educativas de los jóvenes argentinos de distintos grupos demográficos y socioeconómicos? ¿Es mayor la desigualdad de oportunidades en el acceso a calidad que en el acceso a la educación en sí misma? Una vez que se accede al sistema educativo o a cierta calidad educativa, ¿continúa habiendo desigualdad de oportunidades en lo que respecta al desempeño educativo?

Ahora bien, la dificultad de definir un concepto tan complejo como es la desigualdad de oportunidades ha dado lugar a una extensa discusión normativa que atraviesa toda la literatura relacionada, tanto teórica como empírica (Roemer, 1993, 1998, 2004, 2012; Fleurbaey, 2008; Roemer y Trannoy, 2015). Siguiendo a Roemer (1998), el principal exponente de la literatura, una política igualadora de oportunidades es aquella que distribuye los re- 
cursos de forma tal que los individuos que han realizado el mismo grado de esfuerzo para alcanzar un determinado resultado tengan la misma probabilidad de obtenerlo más allá de las circunstancias que cada uno haya tenido que afrontar. Roemer denomina esfuerzo a los factores controlables por los individuos y circunstancias a aquellos factores exógenos que afectan la habilidad de los individuos para alcanzar el resultado respectivo. De esta forma se puede pensar que la igualdad de oportunidades (IOP) surge cuando la distribución de los resultados de los individuos es independiente de las circunstancias.

Sin embargo, al analizar si hay igualdad o desigualdad de oportunidades en la obtención de algún resultado aparecen cuestiones conceptuales a tener en cuenta. Un primer obstáculo es la división de factores entre esfuerzo y circunstancias, la cual no es obvia. En un caso extremo todos los factores podrían ser considerados circunstancias, exógenos al individuo, ya que en principio se podría pensar que una persona no tiene control total de sus genes, gustos, entorno, contexto familiar, etc. Por otro lado, algunas diferencias en resultados debido a las circunstancias podrían ser socialmente aceptadas, como puede ser el caso de las habilidades innatas.

Otros autores distinguen entre factores socialmente aceptables y socialmente no aceptables, en vez de esfuerzos y circunstancias, respectivamente. Checchi y Peragine (2010), por ejemplo, se refieren a una desigualdad "éticamente ofensiva" y "éticamente aceptable". La primera proviene de factores no aceptables mientras que la segunda de factores socialmente aceptables. Gasparini (2002) también utiliza los conceptos de factores aceptables y no aceptables para identificar situaciones injustas. No obstante, tampoco esta clasificación es sencilla ya que es muy probable que no sea objetiva y dependa de las preferencias y juicios de valor de la sociedad en su conjunto. Esto hace que la medición de la desigualdad de oportunidades de resultados no sea trivial y, naturalmente, el caso educativo, no es la excepción.

En los últimos años, parte de la literatura de desigualdad de oportunidades se ha focalizado en operativizar el concepto de desigualdad de oportunidades y proveer mediciones robustas. Sin embargo, aún no existen mediciones sistemáticas del fenómeno. En particular, para Argentina las mediciones de desigualdad de oportunidades son escasas, más aún lo son aquellas que proveen mediciones de desigualdad de oportunidades educativas. Este capítulo busca contribuir con evidencia empírica a partir de la medición de diferentes índices de desigualdad de oportunidades educativas del país para brindar una caracterización de la misma.

Se utilizan microdatos provenientes de la Encuesta Permanente de Hogares (EPH) de los años 2003 a 2012 y del Programa para la Evaluación Internacional de Alumnos (PISA, por sus siglas en inglés) de la OCDE de los años 2006, 2009 y 2012. La EPH permite estudiar la desigualdad de oportunidades vinculadas al acceso o permanencia en la escuela secundaria; así como la desigualdad de oportunidades en el acceso a establecimientos educativos públicos y privados. La segunda fuente, PISA, ofrece información sobre la calidad educativa de ciertos tipos de escuelas a partir del desempeño educativo de los estudiantes en matemática, lectura y ciencias. Además, permite medir la desigualdad de oportunidades del desempeño educativo a partir de las competencias de los alumnos.

En cuanto a las hipótesis del trabajo, es esperable que la desigualdad de oportunidades en el acceso a educación media en general sea baja ya que es posible que la obligatoriedad que impone la Ley de Educación Nacional, los programas de transferencias condicionadas como la Asignación Universal por Hijo (AUH), así como las altas tasas de acceso y finalización de la educación primaria que rigen en el país, disminuyan la influencia de algunas circunstancias de los individuos a la hora de acceder o no a la secundaria. ${ }^{1}$ Mientras que sí

\footnotetext{
${ }^{1}$ Según los datos del SEDLAC (2014) -CEDLAS y Banco Mundial- durante 2003 y 2012 la tasa de acceso al nivel primario asciende a alrededor del $99 \%$ y más del $95 \%$ de los jóvenes entre 15 y 24 años han
} 
se podría esperar un mayor grado de desigualdad de oportunidades en el acceso a calidad educativa. Por ejemplo, si se observa que las escuelas con mayor calidad educativa son las escuelas privadas y asistir a ellas implica un pago de matrícula, el acceso a calidad educativa, en este caso, estaría relacionado con el nivel socioeconómico familiar que podría considerarse un factor exógeno no aceptable. Por otro lado, no es evidente que la desigualdad de oportunidades en el desempeño educativo sea mayor que la del acceso a calidad educativa, ya que la distribución del desempeño educativo puede ser reflejo de factores socialmente aceptables como las habilidades innatas, inteligencia, tiempo dedicado al estudio, entre otros.

A partir de las estimaciones, los resultados muestran que la desigualdad de oportunidades en el acceso a educación media es relativamente baja en Argentina. Además, los diferentes índices indican que esta desigualdad de oportunidades se ha mantenido relativamente estable entre 2003 y 2012. También se encuentra que la desigualdad de oportunidades en el acceso a calidad educativa es más alta que la de acceso en general y que ésta ha aumentado en el tiempo. Se observa un incremento en la desigualdad de oportunidades en el acceso a educación privada y en la desigualdad de oportunidades en el acceso a escuelas que brindan una "calidad educativa integral" (definidas a partir de los puntajes PISA de los países de la OCDE y de América Latina). Por último, a diferencia de lo que se esperaba, el grado de desigualdad de oportunidades del desempeño educativo es bastante alto en comparación al encontrado en otros estudios que abarcan distintos países de América Latina.

En la siguiente sección se detalla la metodología utilizada. En la sección 3 se presenta una descripción de los datos a utilizar. En la sección 4 se muestran los resultados y en la sección 5 se exponen las principales conclusiones del capítulo.

\subsection{Metodología}

\subsubsection{Desigualdad de oportunidades educativas}

Como se mencionó anteriormente, Roemer (1998) clasifica a los factores en esfuerzo y circunstancias. Sin embargo, por motivos de simplificación, se propone catalogar las variables de manera tal de unificar las diferentes clasificaciones que surgen de la literatura. Dado que se pueden asociar los factores que generan desigualdades socialmente no aceptables con variables de circunstancias, es que ambos conceptos se utilizarán indistintamente. Por otro lado, también es posible asociar factores que generan desigualdades aceptables con algún tipo de esfuerzo o elección del individuo, así los factores socialmente aceptables y el esfuerzo también serán considerados en forma indistinta. De esta manera, habrá igualdad de oportunidades (IOP) cuando la distribución de resultados educativos sea independiente de los factores no aceptables (circunstancias).

Una forma de analizar la desigualdad de oportunidades es dividiendo a la población en grupos (Peragine, 2004; Checchi y Peragine, 2005). Para ello, se supone una población finita de alumnos $i=1, \ldots, n$ donde cada alumno $i$ se caracteriza por un conjunto de atributos. Estos atributos consisten en un resultado educativo $y_{i}$, un conjunto de factores no aceptables $X_{i}$ y otro conjunto de factores socialmente aceptables $e_{i}$. Los mismos se pueden escribir en forma matricial donde $y$ es un vector de resultados $n \times 1, X$ es una matriz $J \times n$, donde cada fila representa una variable no aceptable $j$, y $e$ es una matriz $M \times n$, donde cada fila representa una variable aceptable $m$. El método consiste en dividir a la población en grupos, de tal manera que los alumnos de cada grupo presenten las mismas circunstancias $X$. Es decir, que dentro de cada grupo los alumnos tienen las mismas características no aceptables

completado sus estudios primarios en dichos años. 
y solo difieren en términos de los factores aceptables $e$. A su vez, siguiendo a Ferreira y Gignoux (2011) esta partición se puede representar a partir del siguiente conjunto $T$ :

$$
T=\left\{T_{1}, T_{2}, \ldots, T_{H}\right\}
$$

donde $T_{h}$ es el grupo de alumnos $h$ y se cumple que

$$
\begin{aligned}
& T_{1} \cup T_{2} \cup \ldots \cup T_{k}=\{1, \ldots, n\}, \\
& T_{l} \cap T_{k}=\emptyset \text {, para todo } l, k, \text { con } l \neq k, \\
& X_{i}=X_{j} \text { para todo } i, j \text { tal que } i \in T_{h}, j \in T_{h}, \text { para algún } h .
\end{aligned}
$$

La primera condición (1.1) muestra que el resultado de la unión de todos los grupos es el conjunto $T$, que no es más que el conjunto que incluye a todos los alumnos de la población. Además (1.2) especifica que un mismo elemento no puede encontrarse en dos grupos diferentes, por lo tanto, un alumno no puede formar parte de más de un grupo. Por último, (1.3) refleja el hecho que los alumnos de un mismo grupo presentan los mismos factores no aceptables.

Luego, existirá desigualdad de oportunidades entre distintos tipos de alumnos cuando las distribuciones de resultados educativos de dichos grupos sean distintas. Formalmente esto es, existen dos grupos $k$ y $l$ distintos $\left(T_{k} \in T\right.$ y $\left.T_{l} \in T\right)$, tal que

$$
F^{k}(y) \neq F^{l}(y)
$$

donde $F^{k}$ es la función de distribución acumulada de la variable resultado $y$ del grupo $T_{k}$ y $F^{l}$ es la función de distribución acumulada de la variable resultado $y$ del grupo $T_{l} \cdot{ }^{2,3} \mathrm{Al}$ tener en cuenta toda la distribución de resultados de cada uno de los grupos, (1.4) implica adoptar un criterio fuerte de IOP. ${ }^{4}$ Un criterio más débil para IOP consiste en tener en cuenta solo la media de la distribución de resultados, en vez de la distribución completa. En este caso, habrá desigualdad de oportunidades cuando las medias de dos grupos sean distintas. Esto es, existe un par de grupos $k$ y $l$ distintos tal que:

$$
\mu^{k}(y) \neq \mu^{l}(y)
$$

con $\mu^{k}(y)=\int_{0}^{\infty} y d F^{k}(y)$ y $\mu^{l}(y)=\int_{0}^{\infty} y d F^{l}(y)$. En (1.5) al ser las medias de los dos grupos distintas, las respectivas distribuciones también lo son sugiriendo la existencia de desigualdad de oportunidades en sentido débil. Este criterio es consistente con el criterio fuerte porque si

$$
\mu^{k}(y) \neq \mu^{l}(y) \Rightarrow F^{k}(y) \neq F^{l}(y)
$$

De esta manera, rechazar la hipótesis nula de IOP débil es suficiente para también rechazar

\footnotetext{
${ }^{2}$ Matemáticamente, (1.4) corresponde a que $F^{k}(.) \neq F^{l}($.$) , pero por motivos aclaratorios en la redacción$ se opta por especificar el resultado educativo $y$.

${ }^{3}$ Notar que el ranking de esfuerzo $\pi$ y el ranking de la variable de resultado debe ser el mismo dentro de cada grupo: $y^{k}(\pi)=y^{l}(\pi)$.

${ }^{4}$ Cabe señalar que las distribuciones de resultados dentro de cada grupo no son relevantes aquí, debido a que la desigualdad intra-grupos no está relacionada con los factores no aceptables, ya que todos los alumnos de dicho grupo enfrentan las mismas circunstancias.
} 
la hipótesis de IOP en sentido fuerte. Pero al ser un criterio más débil, se puede no rechazar la hipótesis de IOP débil, aún cuando en realidad no hay IOP en sentido fuerte y en este caso este test no es informativo de la hipótesis fuerte. A pesar de esta salvedad, este último criterio puede ser utilizado como un primer test empírico de desigualdad de oportunidades.

\subsubsection{Conjunto de atributos}

Los resultados educativos $(y)$ considerados se basan en tres dimensiones que son: acceso a la educación media, acceso a calidad educativa y desempeño educativo. En el primer caso, el resultado educativo de interés es la asistencia de los jóvenes en edad escolar a la escuela secundaria. La variable de resultado es una variable binaria, que toma valor 1 si el alumno asiste a un establecimiento educativo del nivel medio y 0 si no.

En el segundo caso, el resultado educativo consiste en el acceso a establecimientos educativos que muestren una calidad educativa alta. Aquí se asocia la calidad educativa de un colegio con el desempeño educativo promedio de sus alumnos (para más detalles ver sección 1.3). En este caso también la variable de resultado es una variable binaria que toma valor 1 si se accede a un establecimiento educativo con un determinado nivel de calidad educativa y 0 si no.

Por último, en el caso del desempeño educativo se consideran las competencias de los alumnos, para ello se utilizan los puntajes estandarizados de las pruebas internacionales PISA que brindan información sobre las competencias de lectura, matemáticas y ciencia de los alumnos de 15 años del país. Las competencias son un concepto más amplio que el dominio de currículas educativas y conocimientos alcanzados y están estrechamente relacionadas con el desarrollo de capital humano y la capacidad de los jóvenes para usar los conocimientos, habilidades y destrezas adquiridos. La competencia de lectura se asocia a la capacidad de una persona para comprender y utilizar textos escritos y reflexionar sobre ellos. La competencia de matemática se basa en la capacidad de una persona para identificar y comprender el papel que las matemáticas desempeñan en el mundo y realizar razonamientos bien fundados y, por último, la de ciencias que busca describir la capacidad de utilizar el conocimiento científico de los alumnos.

En cuanto a los factores socialmente aceptables $(e)$ y no aceptables $(X)$ se recurre a la evidencia que surge de la literatura y se consideran aquellos que usualmente se incluyen en los análisis de desigualdad de oportunidades. Las variables aceptables son edad del alumno y año escolar que cursa. Las no aceptables son género, conformación familiar, educación de los padres, ocupación de los padres, estatus migratorio, localización geográfica y recursos educativos en el hogar. Si bien algunas de las variables pueden ser evidentes, otras merecen una explicación más detenida de porqué han sido seleccionadas y caracterizadas de esa forma.

La edad del alumno se caracteriza como un factor aceptable porque se considera que la decisión de asistir o no a un establecimiento escolar si bien, en las etapas de formación más tempranas, es una decisión de los padres la misma pasa a ser una decisión del joven a medida que avanza en edad. Más aún, a pesar de la obligatoriedad de la secundaria es posible que los estudiantes de mayor edad incorporen en su decisión el costo de oportunidad de incorporarse o no al mercado laboral tempranamente. De esta manera los efectos de la edad sobre el acceso pueden considerarse, en principio, aunque no en forma obvia, como aceptables. Por otro lado, la edad se asocia al grado de madurez intelectual y emocional del estudiante. A esto también se suma los requisitos de edad para ingresar a la escuela primaria. Lo que puede provocar que alumnos que difieren en edad por algunos meses, se encuentren en años escolares distintos y aparezcan desigualdades en el desempeño educativo aceptables también. 
El grado que cursa el alumno, hace referencia a si el alumno asiste al grado escolar que le corresponde según su edad. Es más probable que estudiantes que están al día con sus currículas académicas presenten un mejor desempeño en las pruebas respecto de aquellos que están más atrasados siendo estas diferencias aceptables. Además, si un sistema educativo es inclusivo y no expulsa del sistema a los alumnos que repiten el año escolar, en parte está dispuesto a aceptar la desigualdad en el desempeño educativo que surge porque en el aula hay alumnos que han repetido y otros que no. Hay que tener en cuenta que Argentina es un país con altas tasas de repitencia en el nivel secundario (ver Rivas, 2015) y que a esto se le suma, en los últimos años, adaptaciones de sistemas de calificación y asistencia así como cambios en las opciones de cursado y exámenes. Lo que indica que la política educativa presente del país está dispuesta a aceptar la desigualdad en los desempeños educativos que pueden surgir en pos de esta concepción de inclusión educativa.

En cuanto al género, el mismo se considera no aceptable por al menos tres motivos. Primero, porque las normas legales del país manifiestan que todo argentino tiene derecho a acceder a una enseñanza integral independientemente de su género. Segundo, por la necesidad de eliminar en la sociedad los roles tradicionales de género y los estereotipos. Es usual observar que a pesar de que ambos grupos de alumnos, varones y mujeres, están expuestos a los mismos programas de estudios, por un lado, los varones presentan puntajes más altos en matemáticas que las mujeres y, por otro lado, las mujeres presentan puntajes más altos en lectura que los varones y en ciencias las diferencias no son significativas. Este tipo de brechas de género son clave para reducir las diferencias laborales socialmente no aceptables. Tercero, la enseñanza diferenciada por sexo no es frecuente y suele ser mixta en el sistema educativo público del país.

Siguiendo con la ley educativa del país tampoco deberían existir brechas regionales, con lo cual cualquier desigualdad proveniente de diferencias por regiones geográficas es considerada socialmente no aceptable. Para el caso de acceso a la educación media y educación media privada se considera la localización del alumno como variable geográfica y se dividen las regiones en Gran Buenos Aires (GBA), Pampeana, Cuyo, Noroeste Argentino (NOA), Noreste Argentino (NEA) y Patagonia. Mientras que para los demás casos se toma en cuenta la localización del colegio y se supone que la mayoría de los alumnos que asisten a un determinado colegio residen a una distancia no muy lejana del establecimiento. Se dividen las escuelas en aquéllas que se encuentran en un pueblo pequeño, en un pueblo mediano o en una ciudad de más de 100.000 habitantes. Si bien es importante tener en cuenta que en el país existen pueblos pequeños que son pobres y otros que no lo son, así como ciudades grandes pobres y no pobres, cuando se considera como proxy de región geográfica la localización del establecimiento educativo, el análisis se enfoca en las diferencias educativas relacionadas con el tamaño de la región (pueblo vs. ciudad).

La ocupación y educación de los padres están directamente relacionadas con el contexto familiar del alumno. También se consideran tres variables en relación a los recursos educativos del hogar ya que pueden asociarse al entorno del alumno. Estos recursos son computadora, Internet y libros. En particular, la variable libros hace especial referencia a la cultura y la disposición del hogar hacia la lectura y no se refiere a la cantidad de libros en sí. Internet se asocia con la accesibilidad del alumno con el mundo y el conocimiento y la computadora una herramienta tecnológica.

Ahora bien, una vez determinadas las variables aceptables y no aceptables de los individuos, el siguiente paso es armar los grupos de alumnos de manera tal que compartan los mismos factores no aceptables. Sin embargo, esto no resulta una tarea sencilla ya que a medida que aumenta el número de variables no aceptables, la cantidad de grupos aumenta considerablemente, y en varios casos si la muestra no es lo suficientemente grande, el número 
de observaciones de algunos grupos puede ser demasiado pequeño para obtener estimaciones precisas de desigualdad de oportunidades. Por ejemplo, si se considera el género se tienen dos grupos: un grupo de hombres y otro grupo de mujeres. Si luego se agrega una variable más como la educación de los padres que puede ser alta, media o baja, se obtienen 6 grupos (hombre-alta, hombre-media, hombre-baja; mujer-alta, mujer-media, mujer-baja). Si en tercer lugar, se tiene en cuenta la ocupación de los padres (alta, media y baja) se obtienen 18 grupos. Si en cuarto lugar se considera la región geográfica (GBA, NOA, NEA, Cuyo, Patagonia y Pampeana), se obtienen 108 grupos $(18 \times 6)$. De esta manera, por ejemplo, si se tienen 5 variables no aceptables cada una de ellas con 3 posibles valores, tenemos 243 grupos $\left(3^{5}\right)$. Esto hace que la multidimensionalidad sea una importante limitación para la conformación de los grupos.

Una alternativa es trabajar con un número limitado de variables no aceptables. Sin embargo, el análisis se enfocaría solo en algún aspecto del fenómeno. Otra alternativa es seguir un enfoque paramétrico que reduzca los costos de dicha multidimensionalidad.

\subsubsection{Estrategia empírica}

Siguiendo a Bourguignon et al. (2007a) se opta por un enfoque paramétrico, que permite incorporar al análisis un gran conjunto de variables observadas de manera sencilla. Si bien no se trabaja específicamente con grupos de alumnos, la idea que subyace es la de desigualdad de oportunidades en sentido débil (definición (1.5) de la sección 1.2.1).

Formalmente, el modelo propuesto es

$$
\begin{gathered}
y_{i}=\zeta+e_{1, i}^{\prime} \gamma+e_{2, i}^{\prime} \theta+X_{i}^{\prime} \alpha+\varepsilon_{i}, \\
e_{1, i}=X_{i}^{\prime} \delta+v_{i},
\end{gathered}
$$

donde el sub-índice $i$ hace referencia a los alumnos, $y_{i}$ es el resultado educativo del alumno $i$; $e_{i}=\left(e_{1, i}, e_{2, i}\right)$ son las variables aceptables donde $e_{1, i}$ es un vector de orden $M_{1} \mathrm{x} 1$ de variables aceptables asociadas al esfuerzo individual que dependen de variables no aceptables y un término aleatorio $\left(v_{i}\right)$ y $e_{2, i}$ es un vector del resto de las variables aceptables de orden $M_{2} \mathrm{x} 1$. Por su parte, $X_{i}$ es un vector de variables no aceptables de orden $J \times 1, \varepsilon_{i}$ es un término aleatorio y $\delta, \alpha, \theta, \gamma$ y $\zeta$ son vectores de coeficientes. Reemplazando (1.8) en (1.7) se obtiene la forma reducida del modelo:

$$
y_{i}=\zeta+e_{2, i}^{\prime} \theta+X_{i}^{\prime} \beta+u_{i}
$$

donde $\beta$ es $\delta \gamma+\alpha$ y $u_{i}$ es $v_{i} \gamma+\varepsilon_{i}$.

Para los casos donde $y$ es una variable binaria se propone el siguiente modelo de probabilidad no lineal logit,

$$
p_{i} \equiv \operatorname{Pr}\left[y_{i}=1 \mid e_{2, i}, X_{i}\right]=F\left(\zeta+e_{2, i}^{\prime} \theta+X_{i}^{\prime} \beta\right) .
$$

En cuanto a la estimación de los modelos, en el caso del desempeño educativo se estima la ecuación (1.9) a través de Mínimos Cuadrados Ordinarios (MCO) teniendo en cuenta las particularidades de las pruebas PISA (más detalles sobre las mismas se pueden encontrar en el Anexo 1 del capítulo y en el manual de PISA OCDE (2009)). Alternativamente se reemplaza la ecuación (1.9) por un modelo de efectos fijos para incorporar la posible heterogeneidad entre escuelas de la siguiente manera:

$$
y_{i}=\zeta+e_{2, i}^{\prime} \theta+X_{i}^{\prime} \beta+\eta_{s}+u_{i}
$$


donde $\eta_{1}, \ldots, \eta_{S}$ miden los efectos de la heterogeneidad no observada de las escuelas.

En el caso de los modelos de acceso al ser la variable dependiente, $y$, una variable binaria se estiman los modelos de probabilidad no lineal (1.10) por máxima verosimilitud. ${ }^{5}$

Una vez realizadas las regresiones, se estiman los resultados educativos, $\hat{y}$, asignando a todos los individuos las mismas características aceptables $\bar{e}_{2}$. En el caso del modelo lineal estimado por MCO los resultados contrafácticos se obtienen de la siguiente manera:

$$
\hat{y}_{i}=\hat{\zeta}+\bar{e}_{2, i}^{\prime} \hat{\theta}+X_{i}^{\prime} \hat{\beta}
$$

De esta forma las diferencias en $\hat{y}$ vienen dadas por las diferencias en el término de variables no aceptables y sus retornos $\left(X^{\prime} \hat{\beta}\right)$. La desigualdad de la nueva distribución contrafactual, $F(\hat{y})$, corresponde a la desigualdad de oportunidades de $y$ ya que captura solo los efectos de las variables no aceptables, $X$.

Finalmente, para obtener una medida resumen del grado de desigualdad de oportunidades se computa un índice de desigualdad $I$ de la nueva variable de resultados predicha $\hat{y}$. Como índice de desigualdad, se emplea el coeficiente de Gini que es un índice usualmente utilizado en la literatura de distribución. También se considera el coeficiente de Gini absoluto, que es el coeficiente de Gini (relativo) multiplicado por la media de la distribución de resultado cuya desigualdad se está midiendo. También se utilizan la varianza y el desvío estándar (SD) que son medidas de dispersión estadística. ${ }^{6}$ También, en el caso de acceso se incluye como medida de desigualdad, el índice de disimilitud (D), que es una medida de segregación. Este índice se utiliza en la literatura de desigualdad de oportunidades cuando los resultados son variables binarias como la cobertura o acceso a algún servicio (Paes et al., 2008). En todos los casos, valores más altos de los índices implican una mayor desigualdad de oportunidades.

Un problema a tener en cuenta es que los coeficientes estimados de las variables no aceptables $(\hat{\beta})$ podrían no estar mostrando correctamente el efecto de estas variables debido principalmente al problema de variables omitidas. Los mismos podrían estar reflejando una simple correlación y, por lo tanto, no se pueden interpretar como efectos causales. A pesar de este problema, dado que lo que interesa estimar es el efecto conjunto de las variables no aceptables, si todas las variables omitidas se consideran socialmente no aceptables y no están correlacionadas con $e_{2, i}$, el término $X_{i} \hat{\beta}$ daría cuenta sobre la contribución de todos los factores no observables relacionados con las variables no aceptables observadas. Ferreira y Gignoux (2011) argumentan que en este caso es posible encontrar un límite inferior de desigualdad de oportunidades, al estar hallando un efecto de las variables no aceptables sobre $y$ menor que el verdadero. Por ejemplo, si el siguiente vector contiene todas las variables no aceptables del modelo

$$
X_{i}^{T o t}=\left(X_{i}, X_{i}^{*}\right)
$$

y solo se observa un conjunto específico de variables no aceptables $X_{i}$, entonces $\hat{\beta}$ presentaría un sesgo por variables omitidas. Si la correlación entre $X_{i}$ y $X_{i}^{*}$ es negativa, $\hat{\beta}$ sería subestimado (es decir, que $\hat{\beta}$ sería menor al que se obtendría si se observaran todas las variables no aceptables $\hat{\beta}^{\text {Tot }}$ ), y se subestimaría el efecto del conjunto específico de variables no aceptables observadas $X_{i}$ sobre $y$, encontrando una cota inferior. Esta cota inferior junto con un índice de desigualdad de oportunidades monótono creciente, llevaría a encontrar un límite

\footnotetext{
${ }^{5}$ También se estimó un modelo lineal de probabilidad mediante Mínimos Cuadrados Ordinarios (MCO) y se obtuvieron resultados similares a los encontrados con el modelo Logit.

${ }^{6}$ Tanto el coeficiente de Gini absoluto como la varianza y el desvío estándar son medidas invariantes a la estandarización de los puntajes de las pruebas PISA como se explica en Ferreira y Gignoux (2014) (más detalle en el Anexo 1 del capítulo).
} 
inferior de desigualdad de oportunidades. Más aún, la inclusión de variables no aceptables en la regresión no se reduciría y, en general, aumentaría la variación en y y, por lo tanto, la desigualdad de oportunidades aumentaría.

También es posible que la correlación entre $X_{i}$ y $X_{i}^{*}$ sea positiva y se sobreestime el efecto de las variables no aceptables observadas $X_{i}$ sobre $y{ }^{7}$ En este caso, el efecto total de las variables no aceptables $X_{i}^{\text {Tot }}$ continúa siendo un límite inferior pero ahora de todas las variables no aceptables observables y no observables, y, al igual que antes, se estaría en presencia de un límite inferior de desigualdad de oportunidades.

No obstante, lo anterior no es posible sostener si no se puede asegurar que todas las variables omitidas son socialmente no aceptables. Si alguna de las variables no observable en la ecuación (1.9) es socialmente aceptable y está correlacionada positivamente con las no aceptables observadas, existiría un sesgo hacia arriba lo que no llevaría a una cota inferior (Roemer y Trannoy, 2015).

En la esfera educativa no es fácil clasificar a las variables no observables como aceptables o no aceptables. En un extremo, se podría pensar que el individuo no controla todos los factores no observados y, por lo tanto, no es responsable de los mismos y se pueden considerar socialmente no aceptables. Sin embargo, en la secundaria parte de las decisiones y responsabilidades pueden ser atribuidas a los jóvenes y algunas variables no observables podrían ser consideradas aceptables. Ahora bien, suponer la independencia de estas últimas variables con los factores no aceptables es posible ya que si se considera que el individuo es responsable de los resultados de sus acciones es porque dichas decisiones fueron elegidas libremente ("freely chosen effort") y el control sobre las mismas era completo. Si este no fuese el caso, luego no se estarían considerando socialmente aceptables dichas variables no observables. $^{8}$ Dado esto, se asume que las variables socialmente aceptables no observadas no están correlacionadas con las variables no aceptables del modelo y se establece que los resultados de desigualdad de oportunidades aquí encontrados constituyen un límite inferior.

\subsection{Datos}

En cuanto a los datos se trabaja con dos fuentes de información: la base de datos de la EPH de los años 2003 a 2012 y la base de datos PISA de los años 2006, 2009 y 2012. La EPH es una encuesta realizada por el Instituto Nacional de Estadísticas y Censos (INDEC) de la Argentina, es de carácter urbano y representa $2 / 3$ de la población total del país. Provee

\footnotetext{
${ }^{7}$ La omisión de una variable correlacionada con un regresor hace que los estimadores también sean inconsistentes, en este caso de la siguiente manera:$$
\operatorname{plim} \hat{\beta}_{M C O}=\beta+\delta \beta^{*}
$$

con$$
\delta=\operatorname{plim}\left[\left(N^{-1} X^{\prime} X\right)^{-1}\left(N^{-1} X^{\prime} X^{*}\right)\right]
$$

Entonces $\hat{\beta}$ será sobrestimado cuando la correlación entre $X^{\prime}$ y $X^{*}$ sea positiva y el coeficiente $\hat{\beta}^{*}$ de la variable de circunstancia no observable también sea positivo.

${ }^{8} \mathrm{El}$ caso del esfuerzo es diferente, ya que no es posible asegurar que el esfuerzo es independiente de factores no aceptables si se mide en niveles absolutos, ya que es muy probable que las circunstancias de los individuos afecten los niveles de esfuerzo. En la teoría de IOP se propone como alternativa utilizar medidas relativas del esfuerzo considerando el grado de esfuerzo que realizan individuos con similares circunstancias. Es decir, la posición de cada individuo en la distribución de esfuerzo dentro de cada grupo (ver Roemer y Trannoy, 2015).
} 
información socioeconómica de los individuos y de sus hogares. Por otro lado, PISA es un programa internacional que tiene como objetivo evaluar la formación y habilidades de los jóvenes de distintos países del mundo mediante pruebas estandarizadas. Las pruebas PISA se realizan cada tres años a alumnos entre 15 años y 3 meses y 16 años y 2 meses (denominados, en este estudio, alumnos de 15 años) que han terminado al menos 6 cursos de enseñanza obligatoria. La misma tiene una amplia representatividad a nivel nacional pero no local.

Cada una de estas fuentes brinda información diferente. La EPH resulta útil para estudiar el acceso a la educación media en general. El cuestionario contiene la pregunta si asiste o no a algún establecimiento educativo y cuál es el nivel educativo al que asiste; que son insumos para construir la variable de acceso a educación media. También se pregunta si el establecimiento al cual asiste es público o privado. Sin embargo no contiene información sobre otros logros educativos. Por su parte, PISA permite explorar la calidad educativa así como el desempeño educativo de los alumnos de un país a partir de los resultados de las pruebas de lectura, matemática y ciencia. Las evaluaciones están orientadas a captar la capacidad de los jóvenes para utilizar sus conocimientos y destrezas para afrontar los retos de la vida real (OCDE, 2005). Además PISA brinda información representativa de los alumnos que realizan la prueba y reporta datos del contexto familiar y del establecimiento educativo al que asisten.

A partir de las bases de datos de la $\mathrm{EPH}$, se crea la variable asiste que toma valor 1 si el individuo asiste a un establecimiento educativo del nivel medio. Es decir, si asiste al secundario como reflejo del acceso a la educación media. Por otro lado, para explorar la desigualdad de oportunidades en el acceso a calidad educativa se toma como referencia los puntajes de los alumnos que toman las pruebas PISA. Escuelas que exhiben, en promedio, puntajes más altos en las pruebas PISA que otros centros educativos estarían mostrando que sus alumnos tienen una calidad educativa superior respecto de los demás. Así es que se definen dos medidas que proporcionan información sobre la calidad educativa del país: acceso a escuelas privadas y acceso a escuelas de calidad educativa superior.

La Tabla A1.1 del Apéndice 1 muestra que en Argentina, las escuelas privadas, en promedio, presentan puntajes más altos en las pruebas PISA que las escuelas públicas. Por lo tanto, a partir de la EPH se crea la variable binaria asiste_educ_priv que toma valor 1 si el individuo asiste a una escuela privada como proxy de acceso a calidad educativa.

Respecto al acceso a escuelas de calidad educativa superior, se dividen a los establecimientos según el puntaje promedio que presentan. Aquellos establecimientos educativos con puntajes promedios igual o superior a un puntaje determinado $(\bar{y})$ se categorizan como escuelas de mayor calidad educativa. En primer lugar, se toma como umbral el puntaje promedio de los países de la OCDE de cada prueba: $\bar{y}_{O C D E}$. Así se construyen seis variables binarias de acceso a calidad educativa que toman valor 1 si el alumno asiste a un establecimiento educativo cuyos alumnos presentan un puntaje promedio igual o superior a $\bar{y}_{O C D E}$ : 1) en lectura, 2) en matemática, 3) en ciencia, 4) en al menos una de las pruebas cualquiera sea, 5 ) en al menos dos de las pruebas, 6) en las tres pruebas. Y se considera "escuela de calidad integral" a aquella que alcanza un puntaje igual o superior al de la OCDE en las tres pruebas en forma conjunta y se asume que dichos establecimientos proveen una calidad educativa aún mayor.

En segundo lugar, se toma el puntaje promedio de los países de América Latina que participan en el programa PISA (Brasil, Chile, Colombia, México, Perú y Uruguay), $\bar{y}_{L A}, \mathrm{y}$ se construyen seis variables binarias de la misma forma que en el caso de $\bar{y}_{O C D E}$. En tercer lugar, se considera el puntaje promedio de los países vecinos, Brasil, Chile y Uruguay. En cuarto lugar, se tienen en cuenta puntajes vinculados al propio sistema educativo de Argentina y se toman como umbrales los puntajes promedios de los percentiles $75,80,85$ y 90 de 
la distribución de puntajes del país.

Respecto al desempeño educativo, se utilizan los puntajes estandarizados de las pruebas PISA que muestran las competencias que tienen los alumnos de 15 años. ${ }^{9}$ Cabe señalar, que el objetivo de PISA no es medir dichas competencias en forma individual, sino para una población en general. Es por eso que existen algunas particularidades de las pruebas PISA que hay que tener en cuenta al trabajar con estos puntajes. Una de ellas es que de cada prueba se dispone de valores plausibles de los puntajes, es decir, no se tiene un valor puntual para cada alumno sino que PISA estima una distribución de puntajes para cada estudiante y luego toma una muestra aleatoria de 5 valores de esa distribución. Estos últimos son los 5 valores plausibles que brinda la base datos (para más detalles ver el Anexo 1 del capítulo).

En la Tabla 1.1 se definen en forma detallada las variables de resultados de las tres dimensiones educativas y se indica la fuente de información utilizada en cada caso.

\section{Tabla 1.1: Variables de resultado.}

\begin{tabular}{lll}
\hline Nombre & Definición & Fuente \\
\hline $\begin{array}{l}\text { Acceso a educación media } \\
\text { asiste }\end{array}$ & 1 = asiste al secundario & EPH \\
$\begin{array}{ll}\text { Acceso a calidad educativa } \\
\text { asiste_educ_priv }\end{array}$ & $1=$ asiste a una escuela privada & EPH \\
asisteocde1 & $1=$ asiste a una escuela con puntajes promedios igual o superior que el puntaje & PISA \\
& promedio de los países de la OCDE/LA/ARG. en al menos una de las pruebas & \\
asisteocde2 & $1=$ asiste a una escuela con puntajes promedios igual o superior que el puntaje & PISA \\
& promedio de los países de la OCDE/LA/ARG. en al menos dos de las pruebas & \\
asisteocde3 & $1=$ asiste a una escuela con puntajes promedios igual o superior que el puntaje & PISA \\
promedio de los países de la OCDE/LA/ARG. en las tres pruebas & \\
asisteocderead & $1=$ asiste a una escuela con puntajes promedios igual o superior que el puntaje & PISA \\
asisteocdemath & $\begin{array}{l}\text { promedio de los países de la OCDE/LA/ARG. en lectura } \\
\text { promedio de los países de la OCDE/LA/ARG. en matemática }\end{array}$ & \\
asisteocdescie & $1=$ asiste a una escuela con puntajes promedios igual o superior que el puntaje & PISA \\
& promedio de los países de la OCDE/LA/ARG. en ciencias & \\
$\begin{array}{l}\text { Desempeño educativo } \\
\text { lectura } \\
\text { matemática } \\
\text { ciencias }\end{array}$ & $\begin{array}{l}\text { puntajes estandarizados de la prueba } \\
\text { puntajes estandarizados de la prueba } \\
\text { puntajes estandarizados de la prueba }\end{array}$ & PISA \\
\hline
\end{tabular}

En la Tabla 1.2 se definen las variables aceptables y no aceptables y en este caso también se indica la fuente de información. Para la estimación de los modelos de probabilidad de acceso donde las variables dependientes son el acceso a la educación media y el acceso a escuelas privadas, la variable socialmente aceptable $\left(e_{2}\right)$ es la edad del alumno y las variables no aceptables $(X)$ son el género, jefe de hogar en pareja, máxima educación entre el jefe de hogar y su pareja, ocupación del jefe de hogar, estatus migratorio y región geográfica (GBA, Pampeana, Cuyo, NOA, NEA y Patagonia). ${ }^{10}$ En las demás estimaciones cuyas variables dependientes son las variables de acceso a calidad educativa y el desempeño educativo conformadas a partir de los puntajes de las pruebas PISA, las variables aceptables son la edad y el grado que cursa el alumno y las no aceptables son el género, presencia de los padres en

\footnotetext{
${ }^{9}$ Para más detalles sobre la estandarización de los puntajes de las pruebas PISA ver Anexo 1.

${ }^{10}$ Dichas variables surgen de la base de datos de las EPH. En particular, el cuestionario de la EPH solo pregunta por la relación de parentesco con el jefe de hogar. Con lo cual cuando los hogares se conforman por varios miembros no siempre es posible identificar los padres de cada individuo. Por ello se toma la información del jefe de hogar y se asumen conformaciones de hogares simples donde el jefe de hogar y su pareja son los padres de los niños y jóvenes que viven en dicho hogar.
} 
el hogar, máxima educación de los padres, ocupación más alta de los padres, recursos educativos en el hogar, idioma del hogar, estatus migratorio y localización del colegio (ciudad, pueblo mediano y pueblo pequeño).

Tabla 1.2: Variables socialmente aceptables y no aceptables.

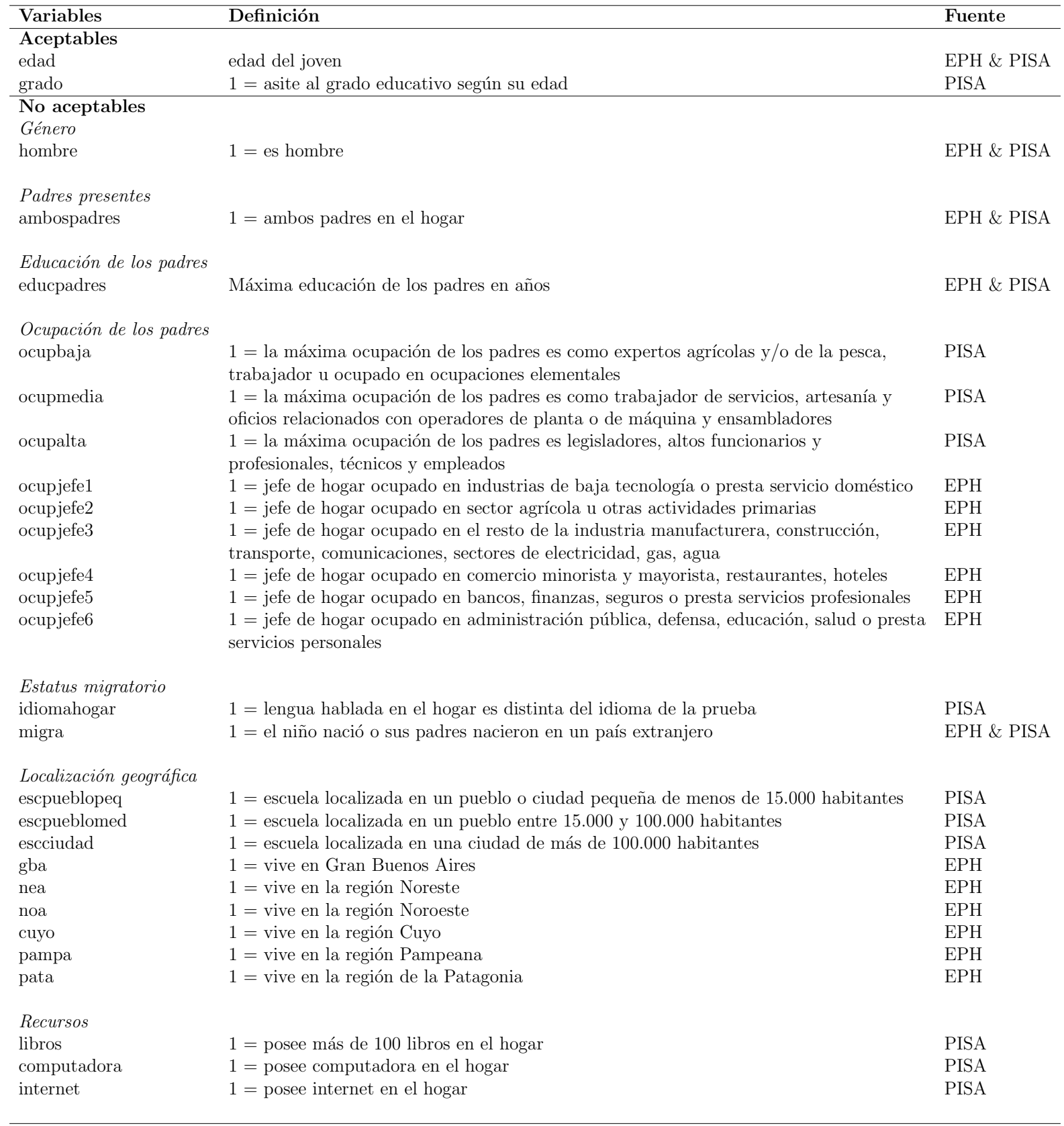

Las estadísticas descriptivas de la muestra de las EPH son reportadas en la Tabla A1.2 y A1.3 del Apéndice 1. La muestra bajo estudio comprende a las personas en edad escolar entre 12 y 18 años que asisten o no a un establecimiento escolar secundario, que han finalizado sus estudios de educación primaria y no se encuentran en un nivel educativo superior (terciario o universitario). También se restringe la muestra principal a una sub-muestra que incluye solamente a jóvenes de 15 años y 16 años, que con motivo de simplificación se denominará muestra de jóvenes de 15 años. Básicamente se crea esta sub-muestra para obtener resultados para este grupo etario, ya que PISA alcanza solo a los jóvenes de 15 años. La cantidad de observaciones de la muestra total varía entre 7.020 en 2006 y 4.055 en 2012. La muestra reducida a jóvenes de 15 años oscila entre 3.250 observaciones en 2006 y 1.755 en 2012. La 
asistencia al secundario de los jóvenes entre 12 y 18 años ha aumentado entre 2003 y 2012 , mientras que el $88 \%$ de los jóvenes asistían a la secundaria en el 2003, este porcentaje pasó a ser $91 \%$ en los últimos años. En el caso de los jóvenes de 15 años los porcentajes son similares.

En cuanto a la base de datos de PISA, se cuenta con un total de 5.908 observaciones en el 2012, y cuando se eliminan los datos faltantes ("missing"), la muestra conserva 4.289 observaciones que representan el $73 \%$ del total de las observaciones. Para 2009 las observaciones totales son 4.474 y las mismas se reducen a 3.908 (82\%) al eliminar las observaciones con datos faltantes. Para 2006 el total de observaciones es 4.339 y se reducen a 3.755 (87\%). Cabe señalar que, tanto en 2006 como en 2009 y en 2012, no se encuentran cambios significativos entre la muestra original y la muestra final. Las respectivas estadísticas descriptivas se reportan en la Tabla A1.4 del Apéndice 1.

Los puntajes promedios de la prueba de lectura de la muestra de 2006, 2009 y 2012 ascienden a 388, 413 y 411, respectivamente; para la prueba de matemática estos puntajes son 391, 400 y 402; y los de la prueba de ciencias son 403, 414 y $420 .{ }^{11}$

\subsection{Resultados}

En esta sección se presentan los resultados de acceso y desempeño educativo. Las estimaciones de los modelos econométricos se reportan en el apéndice. A partir de las mismas se puede observar que los coeficientes estimados que acompañan a las variables explicativas tienen signos y significatividad acorde a lo esperado. Los resultados presentados en la Tabla A1.5 sugieren que es menos probable, ceteris paribus, que los varones asistan a la secundaria. Por otro lado, los jóvenes que tienen ambos padres en el hogar, padres más educados y con mejores ocupaciones laborales presentan más probabilidades de asistir a la secundaria que aquellos que no. Los jóvenes extranjeros tienen menos probabilidad de asistir al colegio. También se encuentran algunas diferencias regionales, aquellos que viven en la Patagonia tienen, ceteris paribus, más probabilidades de acceso que los de GBA, mientras que las demás regiones son las que muestran una menor probabilidad respecto a GBA. Por último, a mayor edad, ceteris paribus, es menos probable asistir a un establecimiento educativo.

La Tabla A1.6 presenta las estimaciones de acceso a educación privada donde, ceteris paribus, es menos probable que los varones y extranjeros asistan a establecimientos privados. Por otra parte, es más probable que jóvenes con ambos padres en el hogar, padres más educados y con mejores ocupaciones laborales asistan a escuelas privadas. Así también aquellos que viven en GBA muestran una mayor probabilidad de acceso. El efecto de la edad, en la mayoría de los casos, es casi nulo o negativo. En cuanto al resto de los resultados de acceso a calidad educativa, las Tablas A1.7 y A1.8 muestran que los varones tienen menos probabilidad de acceder a una calidad educativa alta. Los alumnos que tienen ambos padres en el hogar así como aquellos con padres más educados y ocupados en puestos laborales más altos tienen más probabilidad de acceder a una educación de calidad. A su vez es más probable que los alumnos que disponen de recursos educativos asistan a escuelas con mayor calidad educativa. En cambio pareciera que el estatus migratorio, el idioma del hogar, la localización del colegio y la edad del alumno no afectan las probabilidades de acceso a calidad educativa.

En cuanto al desempeño educativo, las Tablas A1.9 y A1.10 arrojan resultados respecto

\footnotetext{
${ }^{11}$ A pesar del aumento del promedio de los puntajes, a través de los años, Argentina no ha avanzado en el ranking de las pruebas PISA. En 2006 se encontraba en el puesto 51 en matemática y ciencia, 54 en lectura sobre un total de 55 países, mientras que en 2012 se encuentra en el puesto 58 en matemática, 60 en lectura y 57 en ciencias sobre un total de 64 países.
} 
al género que se observan usualmente en la literatura mostrando que las mujeres se desempeñan mejor que los hombres en las pruebas de lectura, los hombres se desempeñan mejor en matemáticas y en el caso de ciencia no se observan diferencias significativas entre ambos grupos. Además padres más educados y ocupación alta arrojan un efecto positivo en el desempeño respecto a los demás padres. Por su parte, la presencia de ambos padres en el hogar no tiene un efecto significativo en el desempeño. Ser extranjero tiene un efecto negativo en el desempeño así como ir a colegios de pueblos pequeños y medianos en relación a los de zonas urbanas más pobladas. La disponibilidad de recursos educativos como libros, computadora e Internet tienen un efecto positivo en el desempeño. También los alumnos de mayor edad y que asisten a un grado escolar de acuerdo a su edad muestran un mayor desempeño.

Una vez estimados los coeficientes de cada regresión, se construyen las respectivas distribuciones contrafactuales de acceso y de desempeño, manteniendo constantes las variables aceptables del modelo. En cada caso, se fija la edad de los jóvenes de acuerdo a su valor promedio reportado en las Tablas A1.2 - A1.4 y, en el caso de desempeño, se indica el valor 1 a la variable grado. Luego, se calculan diferentes índices de desigualdad, con el fin de resumir en una sola medida el grado de desigualdad de oportunidades educativas en el país. A continuación se presentan los índices de desigualdad computados para cada dimensión educativa.

\subsubsection{Acceso a educación media}

En la Tabla 1.3 se presentan los índices de desigualdad de oportunidades para el caso de acceso a educación media en general. Estos resultados corresponden a la muestra de jóvenes entre 12 y 18 años de los años 2003 a 2012. Las mediciones del coeficiente de Gini indican una desigualdad baja. Este índice puede tomar valores entre 0 y 1, donde 0 significa perfecta igualdad y 1 significa completa desigualdad y aquí los valores estimados son menores a 0,1. Se observa que el coeficiente de Gini alcanza un valor máximo en 2008, que asciende a 0,054; mientras que en 2009 disminuye a 0,033 manteniéndose en valores similares durante el resto del periodo. ${ }^{12}$

Por otra parte, el índice de Gini absoluto, alcanza su máximo en 2008 y luego baja manteniéndose relativamente constante en los últimos años en alrededor de 0,032. El desvío estándar también muestra una baja dispersión en la probabilidad de acceso, en los últimos años este índice muestra valores alrededor de 0,060. En cuanto al índice de disimilitud, el mismo se puede interpretar como la fracción de acceso a la educación media que tiene que ser redistribuida desde grupos de jóvenes favorecidos a jóvenes con circunstancias más desfavorables para alcanzar la IOP en el acceso. Este índice alcanza un valor máximo de 0,040 en 2008, indicando que el $4 \%$ del total de las posibilidades de asistir a la secundaria tienen que ser redistribuidas para asegurar que todos los jóvenes cuenten con la misma oportunidad de acceso, señalando así un bajo grado de desigualdad de oportunidades entre los jóvenes para acceder a la educación media.

\footnotetext{
${ }^{12}$ Los valores hallados en este trabajo son menores a los encontrados en Gasparini (2002). El autor analiza la desigualdad en la probabilidad de asistir al secundario condicionada a variables socialmente no aceptables. $\mathrm{Su}$ estudio se enfoca en jóvenes de 13 y 17 años del área de Gran Buenos Aires desde 1980 hasta 1999. Las variables socialmente no aceptables consideradas son el ingreso de los padres, educación máxima del jefe de hogar, género y edad. El valor máximo del coeficiente de Gini que obtiene es 0,193 en los años 1980-1983 y el mínimo ronda en 0,080 durante los años 1998-1999.
} 
Tabla 1.3: Desigualdad de oportunidades en el acceso a educación media.

\begin{tabular}{llllclc}
\hline Año & Gini & \multicolumn{1}{l}{ Interv. Conf. 95 \% } & Gini absoluto & \multicolumn{1}{l}{ Interv. Conf. 95 \% } \\
\hline 2003 & 0.049 & 0.046 & 0.050 & 0.044 & 0.041 & 0.046 \\
2004 & 0.044 & 0.042 & 0.047 & 0.040 & 0.038 & 0.043 \\
2005 & 0.034 & 0.033 & 0.036 & 0.032 & 0.030 & 0.033 \\
2006 & 0.043 & 0.041 & 0.045 & 0.039 & 0.038 & 0.041 \\
2007 & 0.045 & 0.042 & 0.049 & 0.040 & 0.038 & 0.043 \\
2008 & 0.054 & 0.051 & 0.057 & 0.049 & 0.047 & 0.051 \\
2009 & 0.033 & 0.032 & 0.035 & 0.030 & 0.029 & 0.032 \\
2010 & 0.035 & 0.032 & 0.037 & 0.032 & 0.030 & 0.035 \\
2011 & 0.035 & 0.033 & 0.037 & 0.032 & 0.031 & 0.034 \\
2012 & 0.033 & 0.032 & 0.036 & 0.031 & 0.029 & 0.032 \\
\hline Año & Desvío estándar & Interv. Conf. 95 \% & Disimilitud & Interv. Conf. 95\% \\
\hline 2003 & 0.086 & 0.082 & 0.091 & 0.035 & 0.033 & 0.038 \\
2004 & 0.081 & 0.078 & 0.086 & 0.032 & 0.030 & 0.033 \\
2005 & 0.065 & 0.063 & 0.070 & 0.025 & 0.023 & 0.026 \\
2006 & 0.080 & 0.076 & 0.083 & 0.031 & 0.030 & 0.032 \\
2007 & 0.080 & 0.072 & 0.088 & 0.032 & 0.030 & 0.035 \\
2008 & 0.099 & 0.094 & 0.102 & 0.040 & 0.037 & 0.041 \\
2009 & 0.060 & 0.057 & 0.062 & 0.024 & 0.023 & 0.025 \\
2010 & 0.066 & 0.061 & 0.071 & 0.025 & 0.023 & 0.028 \\
2011 & 0.061 & 0.059 & 0.065 & 0.025 & 0.024 & 0.026 \\
2012 & 0.062 & 0.059 & 0.065 & 0.024 & 0.023 & 0.026 \\
\hline
\end{tabular}

Nota: Índices computados a partir de las estimaciones del modelo no lineal Logit. Los datos corresponden al segundo semestre de cada año. Intervalos de confianza estimados por bootstrap (100 reps.).

Fuente: Elaboración propia en base a EPH.

A partir del Gráfico 1.1, que muestra la evolución del índice de Gini durante el periodo 2003 y 2012, se puede observar una tendencia ascendente de la desigualdad desde el 2005 hasta el 2008 y en 2009 una caída del coeficiente, manteniéndose luego relativamente estable. Un comportamiento similar se puede advertir para los demás índices. Esto, en parte, podría estar relacionado con la implementación a fines del año 2009 del programa de transferencia condicionada Asignación Universal por Hijo para la Protección Social (AUH).

La AUH consiste en una transferencia monetaria por hijo menor de 18 años (hasta 5 hijos) a hogares cuyos miembros estén desocupados o sean trabajadores informales. Las condiciones para permanecer en el programa consisten en requisitos sanitarios de inmunización y controles de salud para menores de 4 años y asistencia a algún establecimiento escolar a partir de los 5 años de edad. Por lo tanto, esta transferencia condicionada puede generar un mayor costo de deserción escolar, es decir, si el joven deja de matricularse en el sistema educativo y de asistir a la escuela, la familia no recibe entonces la transferencia de la AUH. Con lo cual, una conjetura que es posible desarrollar es que las dificultades para permanecer en la escuela pueden verse mitigadas, al menos en parte, con la aplicación de este programa y, por ello, es que se encuentra una menor desigualdad de oportunidades en el acceso a partir de 2009. De igual manera, los índices muestran valores pequeños en todos los años. 
Gráfico 1.1: Evolución de la desigualdad de oportunidades en el acceso a educación según coeficiente de Gini.

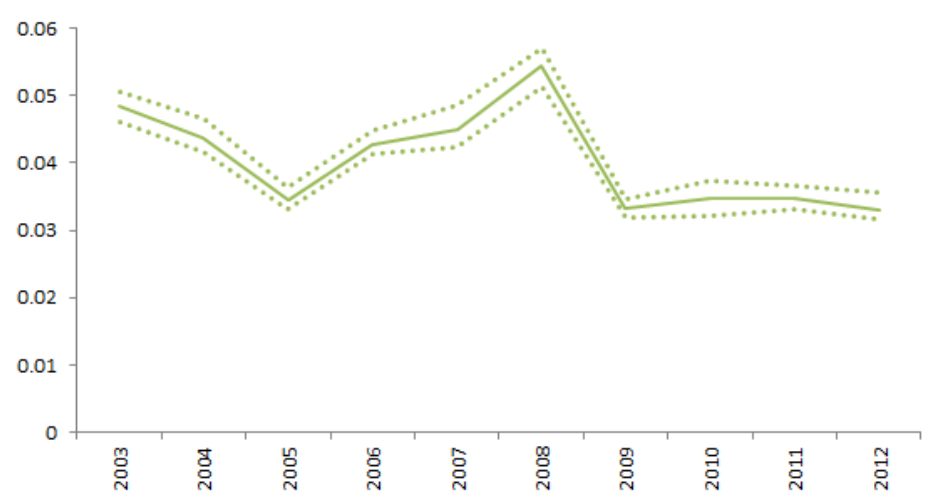

Nota: Líneas punteadas corresponden a intervalos de confianza del $95 \%$

Fuente: Elaboración propia en base a datos EPH.

En el caso de los jóvenes de 15 años, a partir de la Tabla A1.11 del Apéndice 1, se observan valores del coeficiente de Gini más altos que los encontrados para la muestra total, mostrando para este grupo etario una mayor desigualdad de oportunidades. Si se realiza el análisis para el caso de los jóvenes de 12 a 14 años, se encuentra que la desigualdad de oportunidades es mucho menor en estos últimos respecto al grupo de jóvenes de 15 años o más. Esto sugiere que las variables no aceptables aquí definidas se relacionan con la probabilidad de acceso cada vez más a medida que los alumnos avanzan en edad, revelando mayores diferencias entre los alumnos de los últimos años de la secundaria en comparación con los más jóvenes.

A su vez si se realiza este análisis por regiones geográficas, los resultados son similares a los encontrados para Argentina. El Gráfico A1.1 del Apéndice 1 muestra las tendencias del coeficiente de Gini de las regiones GBA, Pampeana, Cuyo, NOA, NEA y Patagonia.

\subsubsection{Acceso a calidad educativa}

En la Tabla 1.4 se presentan los índices para el caso de acceso a educación media privada de los jóvenes de 12 a 18 años. Todos los índices de desigualdad de oportunidades considerados arrojan valores superiores a los reportados en la Tabla 1.3 de acceso en general. El mínimo valor del coeficiente de Gini es 0,335 en el año 2006 y el máximo valor estimado asciende a 0,409 en el año 2012. El índice de Gini absoluto muestra un rango de valores entre 0,093 y 0,123. El desvío estándar muestra una dispersión en la probabilidad de acceso, alrededor de 0,20. El índice de disimilitud empieza en 2003 con un valor de 0,262 y alcanza su valor máximo de 0,310 en 2012. Es decir, que en 2012 aproximadamente el $31 \%$ del total de las posibilidades de acceder a una educación privada tienen que ser redistribuidas desde los alumnos más favorecidos a los menos favorecidos para asegurar que todos los jóvenes cuenten con la misma oportunidad de acceso. Los resultados sugieren que el grado de desigualdad de oportunidades para acceder a una educación privada es alta en comparación con la desigualdad encontrada en el acceso a educación media total. 
Tabla 1.4: Desigualdad de oportunidades en el acceso a educación privada.

\begin{tabular}{lllcccc}
\hline Año & Gini & \multicolumn{1}{l}{ Interv. Conf. 95 \% } & Gini absoluto & \multicolumn{2}{l}{ Interv. Conf. 95 \% } \\
\hline 2003 & 0.357 & 0.349 & 0.368 & 0.105 & 0.102 & 0.108 \\
2004 & 0.343 & 0.335 & 0.350 & 0.103 & 0.099 & 0.107 \\
2005 & 0.357 & 0.349 & 0.365 & 0.109 & 0.105 & 0.113 \\
2006 & 0.335 & 0.324 & 0.340 & 0.107 & 0.103 & 0.110 \\
2007 & 0.344 & 0.336 & 0.356 & 0.093 & 0.089 & 0.096 \\
2008 & 0.351 & 0.344 & 0.358 & 0.106 & 0.103 & 0.109 \\
2009 & 0.369 & 0.361 & 0.377 & 0.110 & 0.107 & 0.114 \\
2010 & 0.359 & 0.347 & 0.365 & 0.115 & 0.112 & 0.119 \\
2011 & 0.391 & 0.381 & 0.401 & 0.116 & 0.112 & 0.122 \\
2012 & 0.409 & 0.396 & 0.419 & 0.123 & 0.118 & 0.127 \\
\hline Año & Desvío estándar & Interv. Conf. 95 \% & Disimilitud & Interv. Conf. 95\% \\
\hline 2003 & 0.188 & 0.181 & 0.194 & 0.262 & 0.255 & 0.272 \\
2004 & 0.186 & 0.179 & 0.191 & 0.253 & 0.244 & 0.262 \\
2005 & 0.198 & 0.192 & 0.204 & 0.260 & 0.253 & 0.266 \\
2006 & 0.191 & 0.185 & 0.198 & 0.245 & 0.238 & 0.250 \\
2007 & 0.166 & 0.161 & 0.173 & 0.257 & 0.248 & 0.264 \\
2008 & 0.188 & 0.182 & 0.193 & 0.261 & 0.254 & 0.267 \\
2009 & 0.197 & 0.192 & 0.204 & 0.272 & 0.263 & 0.277 \\
2010 & 0.206 & 0.196 & 0.212 & 0.265 & 0.257 & 0.273 \\
2011 & 0.210 & 0.203 & 0.217 & 0.293 & 0.286 & 0.302 \\
2012 & 0.220 & 0.213 & 0.228 & 0.310 & 0.299 & 0.321 \\
\hline
\end{tabular}

Nota: Índices computados a partir de las estimaciones del modelo no lineal Logit. Los datos corresponden al segundo semestre de cada año. Intervalos de confianza estimados por bootstrap (100 reps.).

Fuente: Elaboración propia en base a EPH.

A diferencia de lo encontrado anteriormente, los resultados muestran que la desigualdad de oportunidades de acceso a educación privada ha aumentado desde el año 2006. A partir del Gráfico 1.2, se observa un incremento sostenido del coeficiente de Gini en los últimos años, llegando a alcanzar en el 2012 los valores más altos del período. Si se divide el análisis por regiones, una tendencia similar se encuentra para GBA, Cuyo y NOA (ver Gráfico A1.1 del Apéndice 1).

Gráfico 1.2: Evolución de la desigualdad de oportunidades en el acceso a educación privada según coeficiente de Gini.

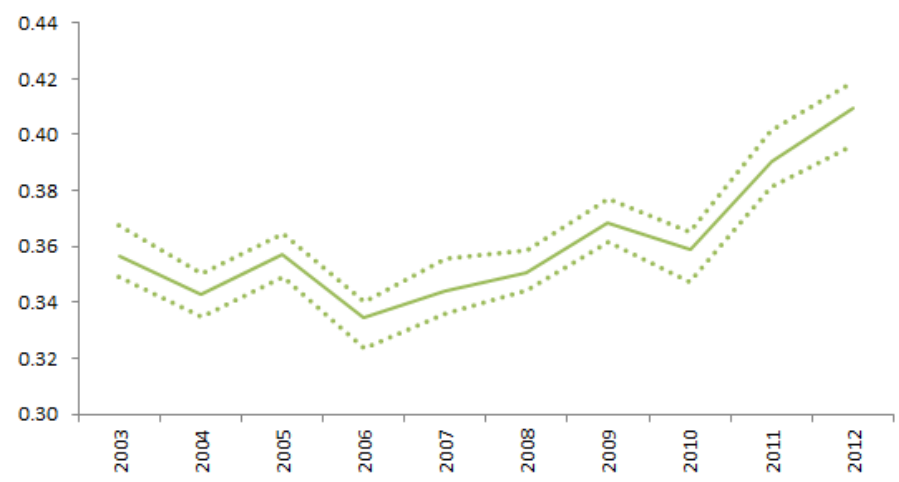

Nota: Líneas punteadas corresponden a intervalos de confianza del $95 \%$

Fuente: Elaboración propia en base a datos EPH. 
Asimismo, cuando se define el acceso a calidad educativa como el acceso a establecimientos con puntajes iguales o superiores al de la OCDE $\left(y_{O C D E}\right)$, se halla una mayor desigualdad a la encontrada anteriormente. El Gráfico 1.3 muestra los resultados del coeficiente de Gini. ${ }^{13}$ Se puede observar que los valores arrojados del coeficiente de Gini se encuentran alrededor de 0,50 y 0,70 . El valor más alto corresponde al acceso a escuelas con puntajes superiores o iguales que $y_{O C D E}^{P}$ en las tres pruebas o a escuelas que lo superan al menos en matemáticas. En este caso el coeficiente de Gini en 2012 arroja los valores 0,69 y 0,66, respectivamente. A su vez, entre 2006 y 2012, la desigualdad de oportunidades en el acceso a escuelas que brindan una calidad integral (puntajes superiores en las tres pruebas) ha aumentado. Este índice pasó de ser 0,63 en el 2006 a 0.69 en 2012.

Inclusive se observa que en 2006 la desigualdad de oportunidades en el caso de al menos una prueba, dos y tres pruebas, así como de cada prueba por separado no variaba demasiado entre sí. En dicho año los coeficientes de Gini oscilan entre 0,62 y 0,65. En cambio, en 2012 los valores son muy diferentes según la definición de calidad educativa que se considere. El coeficiente de Gini alcanza un valor de 0,69 en el caso de las tres pruebas mientras que para una prueba es aproximadamente 0,55. De esta manera la desigualdad de oportunidades es mayor para el acceso a escuelas que ofrecen una calidad integral.

Gráfico 1.3: Desigualdad de oportunidades en el acceso a calidad educativa medida a partir de puntajes de la OCDE según coeficiente de Gini.
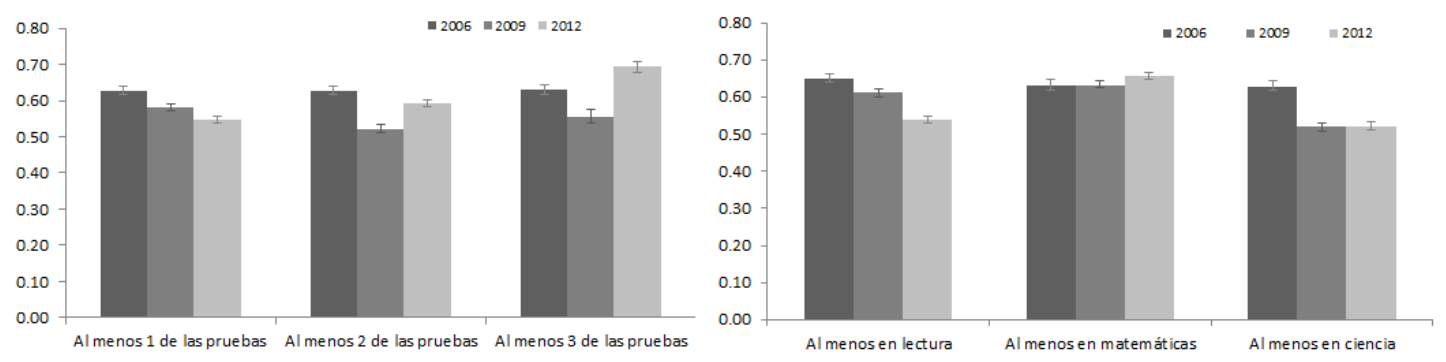

Nota: I son los respectivos intervalos de confianza del $95 \%$ Fuente: Elaboración propia en base a datos PISA.

Cuando se utilizan otras medidas de calidad educativa empleando distintos umbrales de puntajes como el puntaje promedio de los países de América Latina que participan en el programa PISA (Brasil, Chile, Colombia, México, Perú, Uruguay) y el puntaje promedio de los países vecinos, Chile, Brasil y Uruguay, también se encuentra una alta desigualdad de oportunidades en el acceso a una calidad educativa integral. En ambos casos se encontró que los valores más altos del coeficiente de Gini corresponden al acceso a escuelas que presentan puntajes superiores en las tres pruebas en forma conjunta. Asimismo esta desigualdad se ha incrementado entre 2006 y 2012 (Gráfico 1.4).

\footnotetext{
${ }^{13} \mathrm{El}$ resto de los índices se pueden ver en la Tabla A1.12 del Apéndice 1.
} 
Gráfico 1.4: Desigualdad de oportunidades en el acceso a calidad educativa medida a partir de puntajes de América Latina según coeficiente de Gini.
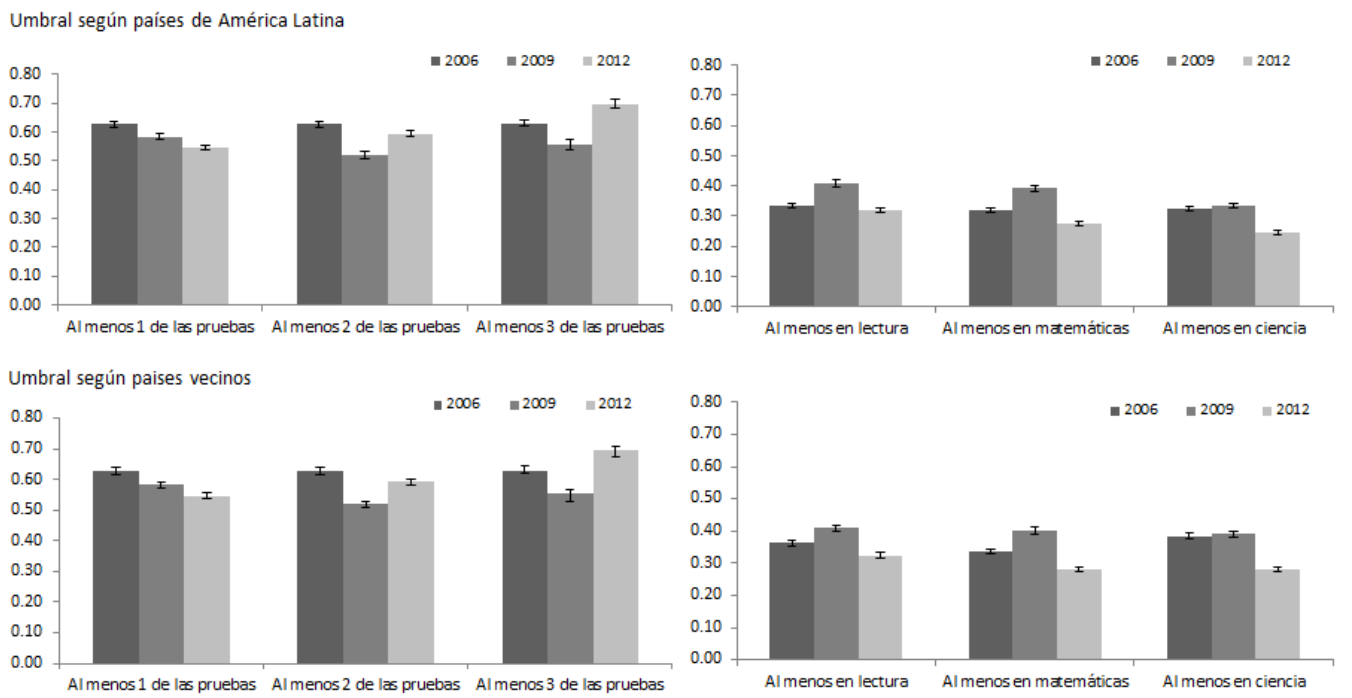

Nota: I son los respectivos intervalos de confianza del $95 \%$.

Fuente: Elaboración propia en base a datos PISA.

Finalmente, cuando se considera una medida de calidad educativa vinculada al propio sistema educativo del país como los puntajes promedios de los distintos percentiles se encuentra que, para los percentiles 75, 80 y 85, la desigualdad de oportunidades ha disminuido entre 2006 y 2012 junto con el valor del umbral (ver Gráfico 1.5). En cuanto al percentil 90, la desigualdad de oportunidades ha aumentado en los casos de al menos dos pruebas y tres pruebas. Nuevamente, esto sugiere que la mayor desigualdad de oportunidades se encuentra en el acceso a una calidad educativa integral. 
Gráfico 1.5: Desigualdad de oportunidades en el acceso a calidad educativa medida a partir de puntajes de Argentina según coeficiente de Gini.
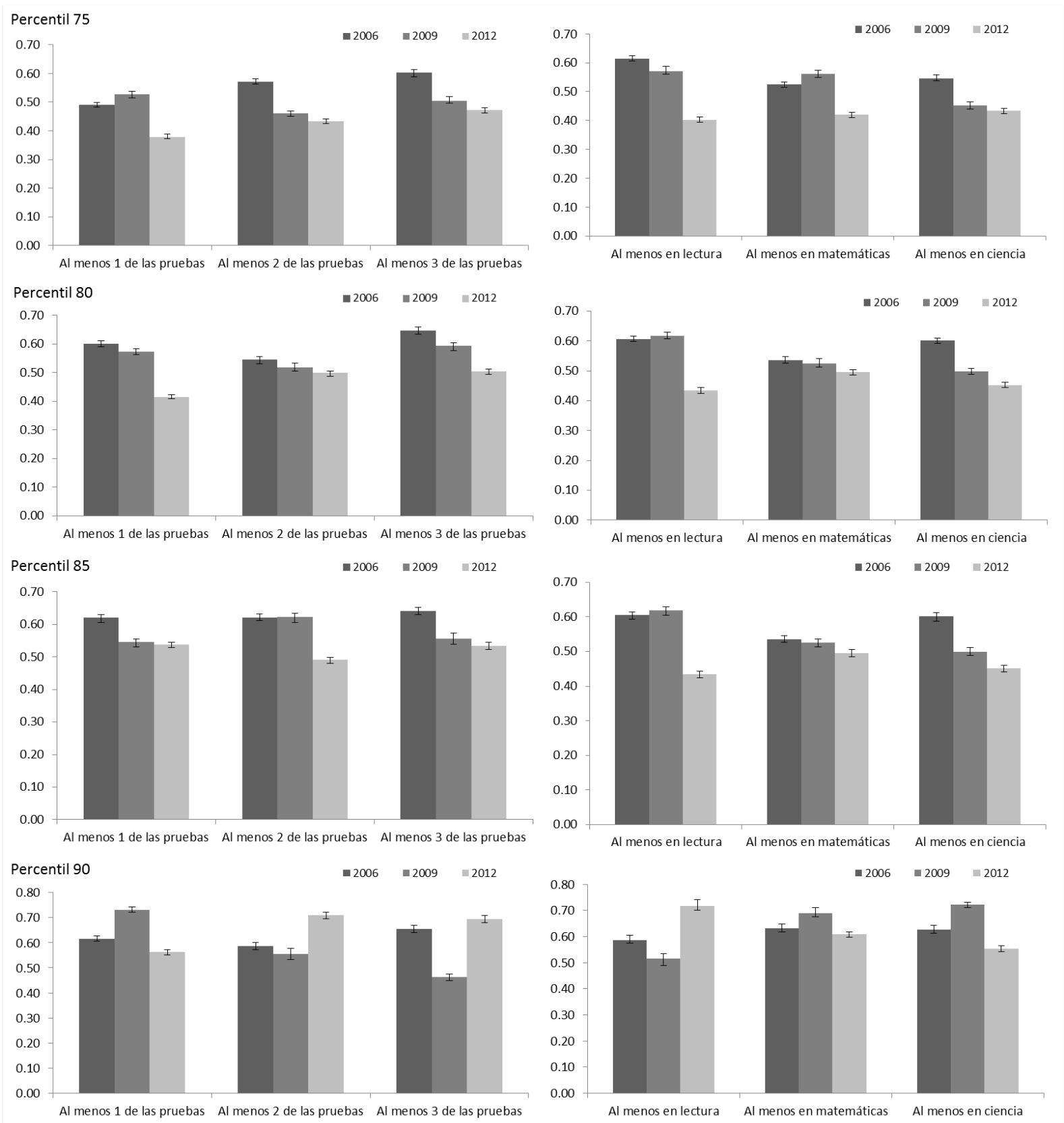

Nota: I son los respectivos intervalos de confianza del $95 \%$. Fuente: Elaboración propia en base a datos PISA.

\subsubsection{Desempeño educativo}

La Tabla 1.5 presenta los índices de desigualdad de oportunidades obtenidos a partir de las distribuciones contrafactuales de desempeño educativo. En la parte (a) se exponen los índices de las distribuciones predichas utilizando los coeficientes estimados por MCO y en la parte (b) se muestran los resultados a partir de los coeficientes estimados por efectos fijos. En la Tabla A1.13 del Apéndice 1 se presentan los respectivos intervalos de confianza. A partir de los índices es posible observar que la prueba de lectura es la que presenta valores más altos sugiriendo una mayor desigualdad de oportunidades en esta competencia. Las circunstancias 
de los alumnos parecen estar más relacionadas con esta competencia que con las demás. Entre otras razones que pueden explicar esta menor desigualdad en ciencias y matemática, se encuentra la dificultad de estas en su enseñanza y aprendizaje. Es muy posible que en el desarrollo de estas competencias las habilidades innatas tengan un rol mayor que el entorno de los alumnos.

Tabla 1.5: Desigualdad de oportunidades en el desempeño educativo.

\begin{tabular}{|c|c|c|c|c|c|c|c|c|c|}
\hline \multirow{2}{*}{$\begin{array}{l}\text { Índice } \\
\text { Prueba }\end{array}$} & \multicolumn{3}{|c|}{ Gini } & \multicolumn{3}{|c|}{ Gini absoluto } & \multicolumn{3}{|c|}{ Desvío estándar } \\
\hline & Lectura & Matemática & Ciencias & Lectura & Matemática & Ciencias & Lectura & Matemática & Ciencias \\
\hline \multicolumn{10}{|c|}{ Parte a: Resultados de las estimaciones por $\mathrm{MCO}$} \\
\hline 2006 & 0.29 & 0.07 & 0.19 & 24.96 & 23.73 & 21.19 & 44.04 & 41.32 & 37.01 \\
\hline 2009 & 0.07 & 0.06 & 0.06 & 26.65 & 24.32 & 24.47 & 46.64 & 42.39 & 42.74 \\
\hline 2012 & 0.06 & 0.04 & 0.04 & 18.10 & 15.78 & 16.19 & 31.87 & 27.65 & 28.36 \\
\hline \multicolumn{10}{|c|}{ Parte b: Resultados de las estimaciones por Efectos Fijos } \\
\hline 2006 & 0.06 & 0.03 & 0.02 & 12.95 & 10.27 & 8.04 & 23.55 & 17.92 & 14.20 \\
\hline 2009 & 0.04 & 0.03 & 0.02 & 11.64 & 9.57 & 9.38 & 20.53 & 16.97 & 16.55 \\
\hline 2012 & 0.03 & 0.02 & 0.02 & 10.60 & 8.60 & 8.72 & 18.70 & 15.22 & 15.54 \\
\hline
\end{tabular}

En cuanto a la proporción de la desigualdad de oportunidades en relación a la desigualdad total. La Tabla 1.6, en primer lugar, presenta la desigualdad de resultados total, es decir el valor del índice de desigualdad de la distribución de puntajes original y, en segundo lugar, presenta la contribución de la desigualdad de oportunidades a la desigualdad total. Dicha contribución se calcula como el cociente entre el valor del índice de desigualdad de oportunidades que proviene de la distribución contrafactual de la Tabla 1.5 y el valor del índice de la desigualdad total.

Tabla 1.6: Participación de la desigualdad de oportunidades del desempeño educativo en la desigualdad total.

\begin{tabular}{|c|c|c|c|c|c|c|c|c|c|}
\hline \multirow{2}{*}{$\begin{array}{l}\text { Índice } \\
\text { Pruebas } \\
\end{array}$} & \multicolumn{3}{|c|}{ Gini } & \multicolumn{3}{|c|}{ Gini absoluto } & \multicolumn{3}{|c|}{ Desvío estándar } \\
\hline & Lectura & Matemática & Ciencias & Lectura & Matemática & Ciencias & Lectura & Matemática & Ciencias \\
\hline \multicolumn{10}{|c|}{ Desigualdad total } \\
\hline 2006 & 0.17 & 0.14 & 0.14 & 66.83 & 55.06 & 54.84 & 118.57 & 98.09 & 97.22 \\
\hline 2009 & 0.14 & 0.13 & 0.13 & 57.14 & 50.62 & 54.44 & 101.29 & 89.84 & 96.63 \\
\hline 2012 & 0.13 & 0.10 & 0.11 & 51.48 & 41.41 & 46.01 & 91.58 & 73.42 & 81.93 \\
\hline \multicolumn{10}{|c|}{ Contribución de la desigualdad de oportunidades } \\
\hline \multicolumn{10}{|c|}{ Parte a: Resultados de las estimaciones por $M C O$} \\
\hline 2006 & $169.19 \%$ & $53.12 \%$ & $139.28 \%$ & $37.35 \%$ & $43.10 \%$ & $38.64 \%$ & $37.15 \%$ & $42.13 \%$ & $38.07 \%$ \\
\hline 2009 & $47.71 \%$ & $48.63 \%$ & $42.78 \%$ & $46.64 \%$ & $48.04 \%$ & $44.94 \%$ & $46.05 \%$ & $47.18 \%$ & $44.23 \%$ \\
\hline 2012 & $48.74 \%$ & $36.12 \%$ & $33.27 \%$ & $35.17 \%$ & $38.11 \%$ & $35.18 \%$ & $34.80 \%$ & $37.66 \%$ & $34.61 \%$ \\
\hline \multicolumn{10}{|c|}{ Parte b: Resultados de las estimaciones por Efectos Fijos } \\
\hline 2006 & $32.66 \%$ & $23.95 \%$ & $14.07 \%$ & $19.38 \%$ & $18.66 \%$ & $14.66 \%$ & $19.86 \%$ & $18.27 \%$ & $14.60 \%$ \\
\hline 2009 & $26.26 \%$ & $22.58 \%$ & $16.76 \%$ & $20.37 \%$ & $18.90 \%$ & $17.23 \%$ & $20.27 \%$ & $18.89 \%$ & $17.12 \%$ \\
\hline 2012 & $20.20 \%$ & $19.85 \%$ & $18.06 \%$ & $20.59 \%$ & $20.76 \%$ & $18.95 \%$ & $20.42 \%$ & $20.73 \%$ & $18.97 \%$ \\
\hline
\end{tabular}

Si se analiza la dispersión de los puntajes a partir del desvío estándar y el índice de Gini absoluto, en casi todos los casos se encuentra que la desigualdad de oportunidades representa más de un tercio de la desigualdad total. ${ }^{14}$ En 2012 la desigualdad de oportunidades medida a través del desvío estándar representa un 34,80\% de la desigualdad de resultados en el caso de lectura, un 34,61\% en el caso de ciencias y un 37,66\% en el caso de matemática. Porcentajes similares también se observan en el caso del índice de Gini absoluto. Estos porcentajes

\footnotetext{
${ }^{14}$ Ferreira y Gignoux (2014) señalan que tanto el desvío estándar como el índice de Gini absoluto son medidas invariantes a la estandarización de los puntajes lo cual conlleva una ventaja respecto a otros índices de desigualdad (ver anexo del capítulo).
} 
resultan ser mayores respecto a los encontrados en otros estudios de desigualdad de oportunidades educativas. Ferreira y Gignoux (2014) analizan la desigualdad de oportunidades de los puntajes de las pruebas PISA de 2006 de 57 países, entre ellos Argentina. ${ }^{15}$ Utilizan el desvío estándar como índice de medición y encuentran una marcada dispersión en las medidas de desigualdad de oportunidades. En países como Australia, Hong Kong y Macau (China) la desigualdad de oportunidades representa alrededor del $10 \%$ y $15 \%$ de la desigualdad total, mientras que en países como Bulgaria, Hungría y Alemania ronda entre el $35 \%$ y $38 \%$. En particular, hallan para Argentina una desigualdad de oportunidades relativa alrededor del $28,9 \%$ en los puntajes de lectura, $31,5 \%$ en los de matemática y $31,2 \%$ en los de ciencias.

Por otro lado, Gamboa y Waltenberg (2012) examinan la desigualdad de oportunidades en seis países de América Latina a partir de datos de PISA 2006 y 2009. Encuentran para Argentina que alrededor del 20-26\% de la desigualdad puede considerarse desigualdad de oportunidades. ${ }^{16}$

En el caso de las estimaciones por efectos fijos se observan valores de índices más bajos a los encontrados por MCO. A pesar de esto, la contribución de la desigualdad de oportunidades a la desigualdad total es alrededor del $14 \%$ y $20 \%$ para el caso del índice de Gini absoluto y desvío estándar. A su vez se encuentra que el componente de error fijo de las escuelas representa gran parte de la desigualdad de los puntajes de las pruebas de los estudiantes (entre el $30 \%$ y el $45 \%$ ). En principio, no se tiene en cuenta este efecto porque se asume que los alumnos tienen libertad para trasladarse entre las escuelas. Si bien el número de escuelas entre las que pueden desplazarse es limitado, la distribución de estudiantes entre las escuelas no es rígida. Incluso en ciertas ocasiones el ingreso a una escuela puede estar relacionado con cantidad de horas estudiadas para los exámenes de ingreso, disciplina del alumno, responsabilidad, todas características relacionadas a variables aceptables y controlables por el alumno. No obstante, si se tuviera en cuenta este componente de error la desigualdad de oportunidades pasaría a representar gran parte de la desigualdad total (alrededor del $70 \%$ ).

En cuanto a la evolución de los índices, el Gráfico 1.6 muestra los desvíos estándar de los puntajes contrafácticos estimados a partir de MCO. El gráfico de la izquierda muestra una disminución de la desigualdad de oportunidades en valores absolutos entre 2006 y 2012. En el caso de lectura el desvío estándar disminuyó 13 puntos, en el caso de matemática 11 puntos y en ciencia 9 puntos. Por otro lado, la participación relativa de la desigualdad de oportunidades en la desigualdad total medida por el desvío estándar, también descendió en 2012 respecto a 2006 pero solo alrededor de 3 puntos porcentuales en el caso de lectura, 5 puntos porcentuales en matemática y 4 puntos porcentuales en ciencia, mostrando así una caída relativa menor (ver der. Gráfico 1.6).

\footnotetext{
${ }^{15}$ Las variables de circunstancias que consideran los autores son el género, educación de la madre, educación del padre, ocupación del padre, idioma del hogar, estatus migratorio, acceso a libros en el hogar, posesión de bienes durables en el hogar, ítems culturales de la familia y la localización de la escuela.

${ }^{16}$ En este caso, los autores utilizan como variables de circunstancias el género, la educación de los padres y el tipo de escuela.
} 
Gráfico 1.6: Desvío estándar de los puntajes de las pruebas PISA de las distribuciones contrafactuales estimadas por MCO.
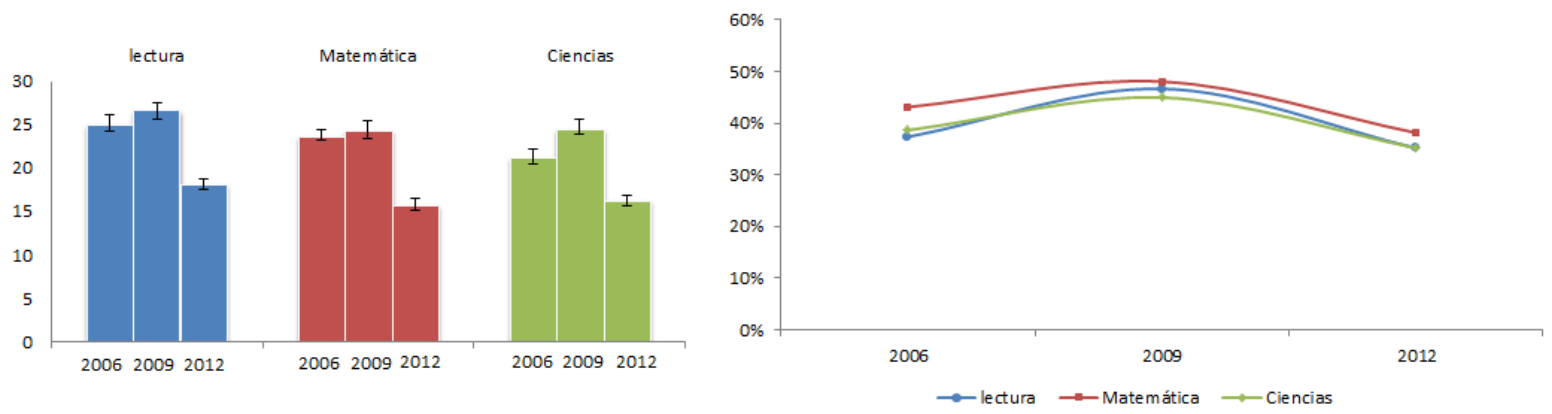

Nota: I son los respectivos intervalos de confianza del $95 \%$. Fuente: Elaboración propia en base a datos PISA.

Por último, en el caso de las estimaciones por Efectos Fijos, el Gráfico 1.7 muestra una disminución en los valores de los desvíos estándar de las pruebas de lectura y de matemática, entre 2006 y 2012, mientras que para ciencias se encuentra un incremento. Si se observa la proporción relativa de la desigualdad de oportunidades en la desigualdad total, la misma ha aumentado entre 2006 y 2012 para las tres pruebas.

Gráfico 1.7: Desvío estándar de los puntajes de las pruebas PISA de las distribuciones contrafactuales estimadas por Efectos Fijos.
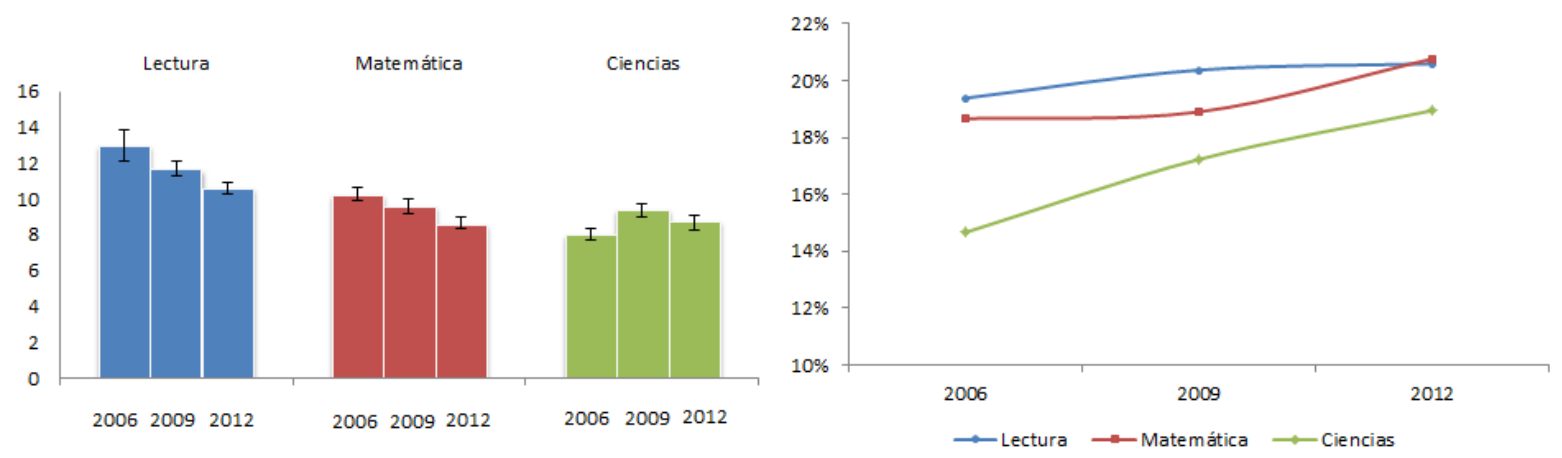

Nota: Desvíos estándar computados a partir de las estimaciones según Efectos Fijos. I son los respectivos intervalos de confianza del $95 \%$. Fuente: Elaboración propia en base a datos PISA.

\subsection{Conclusiones}

Las oportunidades educativas ocupan un lugar central en el conjunto de posibilidades de un individuo. La desigualdad de oportunidades educativas es una de las primeras limitaciones para mejorar las opciones de vida y atenta contra la inclusión y movilidad social. Pese a esto, son pocos los estudios que brindan evidencia del grado de desigualdad de oportunidades educativas y aún no existen mediciones sistemáticas de dicho fenómeno. Este documento constituye un primer esfuerzo en esta dirección.

En este capítulo, se analizó la desigualdad de oportunidades educativas en Argentina desde tres dimensiones: acceso a educación media, acceso a calidad educativa y desempeño educativo. El modelo de desigualdad de oportunidades propuesto consiste en dividir los factores asociados a un resultado educativo en socialmente aceptables y no aceptables. En 
particular, el interés reside en las distribuciones contrafactuales de los resultados educativos que surgen cuando los individuos solo se diferencian en los factores no aceptables. La desigualdad proveniente de cada distribución contrafactual se asocia a la desigualdad de oportunidades que existe para alcanzar el respectivo resultado educativo.

En cuanto a las variables utilizadas, se consideraron aceptables la edad del joven y el grado que cursa y no aceptables la educación de los padres, la ocupación de los padres, la conformación familiar, el género, el idioma del hogar, el estatus migratorio, la posesión de recursos educativos en el hogar y la localización geográfica. A los efectos de obtener las diferentes distribuciones contrafactuales, en primer lugar, se estimaron modelos de probabilidad no lineal logit de acceso a educación y acceso a calidad educativa y, en segundo lugar, se estimaron modelos lineales de desempeño educativo. Luego, se computó el grado de desigualdad de dichas distribuciones a partir de los índices de Gini, desvío estándar y disimilitud.

Los resultados sugieren que la desigualdad de oportunidades encontrada en el acceso a educación media no sólo es baja sino que la misma se ha mantenido relativamente estable en los últimos años. En cuanto a calidad educativa, en los últimos años, se encuentra una escasa mejora en las oportunidades en el acceso a calidad educativa del nivel medio. La mayor desigualdad de oportunidades se da para el caso de acceso a escuelas que ofrecen una calidad educativa integral. Respecto a las distribuciones de desempeño, las mediciones sugieren que si bien la desigualdad de oportunidades de los puntajes ha disminuido entre 2006 y 2012, la misma es alta si se considera la contribución de la desigualdad de oportunidades a la desigualdad total. Lo que sugeriría que el desarrollo de las competencias de los estudiantes están estrechamente correlacionadas con las variables no aceptables tal cual han sido definidas en este trabajo.

De los resultados se infiere que uno de los principales desafíos del país, en pos de las oportunidades educativas, es no solo garantizar el acceso a la educación media sino también el acceso a calidad educativa y, de esta manera, disminuir las disparidades en relación a este resultado educativo. En los últimos años, la desigualdad de oportunidades en el acceso a calidad educativa ha aumentado, indicando que las oportunidades educativas son cada vez más dispares entre los alumnos que logran acceder a escuelas que brindan una calidad educativa integral y aquellos que no.

Por último, cabe señalar que no es posible interpretar los coeficientes estimados de los modelos como efectos causales sin algunos supuestos sobre los residuos. Específicamente se asume que las variables aceptables no observables no están correlacionadas con las variables no aceptables incluidas en el modelo. Por lo que, resulta relevante evaluar la factibilidad del supuesto y la robustez de los resultados. En principio, se entiende que lo que se obtiene en el trabajo es una medida del límite inferior de la desigualdad de oportunidades. Con lo cual si el límite que se encuentra ya es alto, esto es una señal que en dicha dimensión la desigualdad de oportunidades es significativa. Otra cuestión a profundizar es que se trabaja con un criterio débil de desigualdad de oportunidades. Este estudio se enfoca en la esperanza condicional de los resultados educativos $E(y \mid e, X)$. El paso lógico es extender el estudio a otros momentos de la distribución así como a otros métodos de estimación y medidas de desigualdad de oportunidades. Algunos de ellos se abordan en el capítulo siguiente. 


\section{CAPÍTULO 2}

\section{Cambios distributivos en el desempeño educativo. Evidencia de desigualdad de oportunidades a partir de un enfoque de regresiones de funciones de influencia recentradas.}

\subsection{Introducción}

La desigualdad de resultados suele despertar cierta preocupación cuando proviene de un proceso socialmente no aceptable. Ciertos factores pueden originar o fomentar dicho proceso produciendo desigualdad de oportunidades mientras que otros pueden generar una desigualdad aceptable. Por lo tanto, resulta importante conocer cuáles son los factores subyacentes de la distribución de resultados y cuál es la contribución de cada uno con el fin de dirigir políticas distributivas que desarticulen dicho proceso y reduzcan la desigualdad generada. A su vez es relevante conocer cómo dicha contribución ha aumentado o disminuido la desigualdad total. En particular, si los factores no aceptables han contribuido a aumentarla entonces es signo de que la desigualdad de oportunidades se ha acrecentado independientemente del cambio global de la desigualdad total.

Este capítulo estudia los cambios en la desigualdad de oportunidades educativas en Argentina detallando en forma directa cómo cada variable no aceptable ha contribuido a la desigualdad total en los últimos años. Se propone identificar cambios en la distribución de resultados $F(y)$ explorando los aportes individuales de cada variable en distintos puntos de la distribución, lo cual es atrayente cuando se espera que el efecto de un factor sea distinto en diferentes partes de la distribución. Esto permite poder extender el análisis a otras medidas distributivas diferentes a las medidas centrales y observar cambios individuales sobre la desigualdad. Son pocos los trabajos en la literatura que aportan estimaciones del aumento (o disminución) de la desigualdad de oportunidades a partir de este tipo de análisis y esta es la principal contribución de este capítulo.

El objetivo principal consiste en comprender el rol de distintos factores en el cambio distributivo del desempeño educativo de los jóvenes de Argentina a lo largo de la última década. En particular, el análisis se enfoca en la distribución de los puntajes de matemáticas de las pruebas PISA. Se consideran factores socialmente aceptables a la edad del alumno y al año escolar y factores socialmente no aceptables a las características de los padres, el género, la región y los recursos educativos del hogar. De esta manera, si se encuentra que los factores no aceptables han contribuido a aumentar (disminuir) la desigualdad de los punta- 
jes de matemáticas, entonces la desigualdad de oportunidades educativa habrá aumentado (disminuido) en el periodo. Ahora bien, como ya se ha mencionado, la distinción de factores entre aceptables y no aceptables no está exenta de dificultades ya que depende de juicios de valor. Siguiendo a Roemer (1998); Betts y Roemer (2006) los logros educativos (en este caso el desempeño educativo) no deberían depender de factores que la sociedad considera que no son responsabilidad del individuo, usualmente estos factores son circunstancias del individuo como la condición socioeconómica de los padres, la región de origen y el género. Dichos factores son los considerados para este análisis y son los que se suelen encontrar en la literatura. Los efectos de estos factores sobre el desempeño educativo son los que interesan para poder brindar información para el diseño de intervenciones de políticas tendientes a reducir o eliminar la desigualdad de oportunidades.

Las descomposiciones son un instrumento analítico utilizado bajo distintos enfoques y metodologías que permiten estimar los aportes de cada factor individual en la distribución de resultados. ${ }^{1}$ Si bien el uso más extendido de estas técnicas se enfoca en las brechas salariales, su uso es amplio en la literatura de distribución y se puede extender al análisis de desigualdad de oportunidades. Por ejemplo, Checchi y Peragine (2005, 2010) utilizan técnicas de descomposición por grupos para analizar la desigualdad de oportunidades de ingresos en Italia. En base a este método Gamboa y Waltenberg (2012) computan la desigualdad de oportunidades educativas de 6 países de América Latina utilizando los puntajes de las pruebas PISA. Por otro lado, Bourguignon et al. (2007a); Ferreira y Gignoux (2011), entre otros, estiman el aporte individual de cada variable, a partir de distribuciones contrafactuales de ingresos que se simulan manteniendo constante todas las variables menos la de interés. Ferreira y Gignoux (2014) analizan la desigualdad de oportunidades educativas y llevan a cabo una descomposición de valor de Shapley de la varianza propuesta por Shorrocks (1982).

En este capítulo se utiliza un método de descomposición novedoso propuesto por Firpo et al. $(2007,2009)$ que emplea regresiones de funciones de influencia recentradas (RIF, por sus siglas en inglés). Algunos trabajos han aplicado el método de descomposición de RIF para Argentina con el fin de analizar efectos distributivos en ingresos y salarios. Alejo et al. (2014) utilizan regresiones de cuantiles no condicionados para analizar los efectos distributivos de la educación sobre los ingresos. Beccaria et al. (2014) estudian los efectos de la formalización laboral en la distribución de salarios del país. Fabris y Montes-Rojas (2014) examinan los determinantes de los cambios en la distribución del ingreso en Argentina de las últimas dos décadas. Este método resulta ser lo bastante general y flexible para aplicarlo también al caso de desigualdad de oportunidades educativas.

Una ventaja de este método es que admite descomposiciones detalladas para conocer la contribución de cada variable en forma individual sin desviarse del objetivo que persigue el método de Oaxaca-Blinder. Mientras que la clásica descomposición de Oaxaca-Blinder se concentra en descomponer las diferencias de media entre dos grupos y conocer la contribución individual de cada variable sobre dicha diferencia, la descomposición por regresiones de RIF permite examinar en forma detallada otros aspectos de la distribución más allá de la media como la varianza, el coeficiente de Gini y brechas de cuantiles. ${ }^{2}$

El análisis distributivo abarca el período 2000-2012 y luego abarca los cambios distributivos de los sub-periodos 2006-2012 y 2009-2012. Las estimaciones de las regresiones de RIF sugieren efectos heterogéneos a través de los cuantiles. Mostrando que algunas variables tienen efectos sobre las brechas de cuantiles y no sobre la varianza o el coeficiente de Gini. Cuando se analiza la evolución de la desigualdad de los puntajes, los distintos índices arrojan

\footnotetext{
${ }^{1}$ Fortin et al. (2011) presentan una síntesis de los principales métodos existentes.

${ }^{2}$ En el caso de la media la descomposición por RIF resulta ser similar a la de Oaxaca-Blinder cuando se cumplen ciertos supuestos específicos. Ver Firpo et al. (2007) para más detalle.
} 
que la desigualdad cayó entre 2000 y 2012. Las descomposiciones sugieren que, en principio, la disminución de la desigualdad de los puntajes correspondería al término residual y a los retornos de las variables aceptables. Cuando se analizan los efectos de las variables socialmente no aceptables definidas en este estudio, se encuentra que entre 2000 y 2012, en general, estos factores no contribuyeron fuertemente a disminuir la desigualdad de oportunidades.

Por último, conocer en qué medida los factores impactan en la distribución de resultados es relevante para entender el alcance de la desigualdad de oportunidades y para el diseño de políticas redistributivas que tengan por finalidad eliminar este tipo de desigualdad. La identificación de factores manejables por las políticas educativas permite conocer el impacto distributivo potencial de los mismos y diseñar medidas complementarias o compensatorias para aquellos factores que no son manejables. Por ejemplo, algunas intervenciones como los programas de niñez temprana (Early Childhood Development Programs, ECD), las clases extracurriculares, los espacios de estudio y los recursos educativos en las aulas, pueden complementar el déficit educativo familiar .

Las siguientes secciones se organizan de la siguiente manera: primero se presenta la metodología a utilizar, en la sección 3 se describen los datos y en la sección 4 se exhiben los principales resultados. Finalmente, en la sección 5 se presentan las principales conclusiones del capítulo.

\subsection{Metodología}

\subsubsection{Método de descomposición a partir de regresiones de RIF}

En el Capítulo 1 se utiliza una metodología en base a un criterio débil de desigualdad de oportunidades a partir de diferencias de medias y se trabaja principalmente con la función de esperanza condicional de la variable de resultado $y, E(y \mid X)$. En este capítulo se utiliza una metodología que permite examinar los efectos individuales de las variables aceptables y no aceptables en distintos puntos de la distribución de resultados. La principal diferencia con el capítulo anterior es que la metodología utilizada aquí permite ir más allá de las medidas centrales de la distribución. Esto es relevante especialmente cuando se espera que el efecto de algunas variables sobre el resultado sea diferente en distintas partes de la distribución. En el caso educativo es probable que el efecto de algunas variables sobre los puntajes de las pruebas de matemáticas sea diferente en los cuantiles más bajos de la distribución que en los cuantiles más altos. Por ejemplo, si el idioma principal del hogar del alumno es diferente al de la prueba, el mismo puede afectar negativamente el desempeño del alumno, pero es probable que afecte en menor medida a aquellos alumnos que les va muy bien en la prueba ya que puede ser que este desempeño mayor se vincule más con el talento y habilidades para las matemáticas y menos con el idioma. Por lo tanto, sería esperable que el efecto del idioma del hogar sea menor en los cuantiles más altos que en los cuantiles más bajos de la distribución de puntajes.

Este capítulo se centra en explorar los efectos distributivos individuales de las variables aceptables y no aceptables en la distribución del desempeño educativo en la última década. Para ello se realizan microdescomposiciones aplicando el método propuesto por Firpo et al. (2007, 2009), que se puede también encontrar en Fortin et al. (2011). Estas microdescomposiciones utilizan como insumo las estimaciones de las regresiones de las funciones de influencia recentradas de Firpo et al. (2009).

Las regresiones de RIF se basan en la función de influencia y consisten en regresar una transformación de la variable de resultado $Y$ en función de las variables explicativas $X$. Con motivo de simplificación, en esta sección, el conjunto de las variables $X$ incluye todas las va- 
riables explicativas del modelo de desigualdad de oportunidad, ya sean variables socialmente aceptables como variables socialmente no aceptables.

La transformación de la variable $Y$ consiste en la función de influencia recentrada (RIF). Esta función es la suma del estadístico de interés de la distribución $(v)$ que se quiere analizar y la respectiva función de influencia (IF). Es decir,

$$
R I F(Y ; v)=v+I F(Y ; v)
$$

donde $v$ es cualquier estadístico para el cual se puede calcular una función de influencia, algunos ejemplos son los cuantiles, la varianza y el coeficiente de Gini (ver Anexo 2 para más detalle de la regresiones de RIF para estos casos). La IF de una distribución estadística mide el efecto relativo de una pequeña perturbación en la distribución subyacente en el estadístico de interés. Dado que el valor esperado de la IF es igual a cero, la esperanza de la RIF devuelve a $v:^{3}$

$$
\int R I F(Y ; v) d F(Y)=\int(v+I F(Y ; v)) d F(Y)=v
$$

donde $F($.$) es la función de distribución acumulada de Y$.

Entonces aplicando esperanzas iteradas a la RIF se obtiene la siguiente regresión:

$$
m^{v}(X)=E(R I F(Y ; v) \mid X)
$$

Las regresiones se pueden usar para estimar el efecto de un cambio pequeño de la distribución de $X$ en $v$ o para proveer una aproximación de primer orden de un cambio grande (conocido como policy effect). Dado que el método se basa en cambios infinitesimales, las regresiones de RIF solo proveen una primera aproximación del efecto de las covariables cuando el cambio en la distribución de las mismas es grande (ver Firpo et al., 2009). En principio, no hay una regla para determinar si el cambio es grande o pequeño. Y como toda aproximación, será buena dependiendo de cuán grande sean los errores de aproximación. En los casos donde los errores sean considerables, se podría estar cometiendo un error de estimación.

Estas regresiones de RIF pueden especificarse en forma lineal para $k$ variables de la siguiente manera:

$$
m^{v}(X)=X \gamma^{v}
$$

donde $X$ es una matriz de variables de orden $n \times K$ y $\gamma^{v}$ es un vector de coeficientes de orden $K \times 1$. Para el caso de los cuantiles, la regresión de RIF se la conoce como Regresión de Cuantiles No Condicionados. Esto no quiere decir que no se tienen en cuenta las covariables del modelo, sino que los cuantiles corresponden a los cuantiles de la distribución marginal de la variable de resultado.

Respecto a las microdescomposiciones, el objetivo principal es analizar los cambios distributivos de $Y$ entre dos grupos $G$. El uso de regresiones de RIF en las microdescomposiciones permiten una generalización del método de Oaxaca-Blinder ya que es posible extender el

\footnotetext{
${ }^{3}$ Esta función es una medida robusta de $v$ cuando $F$ es reemplazada por su distribución empírica.
} 
análisis de descomposición a otros funcionales $v$ de la distribución distintos de la media. Al igual que en Oaxaca-Blinder los grupos pueden referirse a dos momentos del tiempo $\left(t_{0}, t_{1}\right)$. En dicho caso, el método provee información sobre los cambios de $v$ ante cambios en la distribución de una covariable $X_{k}$ de $t_{0}=0$ a $t_{1}=1$, manteniendo la distribución de las demás covariables constantes. Esta es una de las principales ventajas respecto a otros métodos de descomposición de más allá de la media (como los métodos de Juhn et al., 1993; DiNardo et al., 1996; Machado y Mata, 2005) ya que con este método es posible analizar el impacto individual de cada variable $X_{k}$ sobre el estadístico.

La descomposición al igual que la de Oaxaca-Blinder se realiza en dos etapas, una primera etapa donde se descomponen los cambios distributivos en: un componente de composición (conocido como "composition component") y otro componente de parámetros ("structure component"). En la segunda etapa, se computa la contribución individual de cada variable utilizando las funciones de influencia recentradas.

En la primera etapa se tiene que el cambio total de $v, \triangle_{O}^{v}$, entre dos grupos $G=0,1$ es:

$$
\triangle_{O}^{v}=v\left(F_{1}\right)-v\left(F_{0}\right)=v_{1}-v_{0}
$$

donde $F_{1}$ es la distribución del resultado $Y$ del grupo 1 y $F_{0}$ es la distribución de $Y$ del grupo 0. Ahora la distribución de la variable de resultado $Y$ surge bajo la estructura y la distribución de las características observables $X$ y no observables $u$ de cada grupo. Entonces se puede generar una distribución contrafactual $F_{C}$ que surja bajo la estructura del grupo 0 , pero con la distribución de las características observables y no observables del grupo 1.

Al sumar y restar el funcional de la distribución contrafactual a la diferencia global, ésta se puede descomponer en dos partes:

$$
\triangle_{O}^{v}=\left(v\left(F_{1}\right)-v\left(F_{C}\right)\right)+\left(v\left(F_{C}\right)-v\left(F_{0}\right)\right)=\triangle_{S}^{v}+\triangle_{X}^{v},
$$

donde $\triangle_{S}^{v}=v\left(F_{1}\right)-v\left(F_{C}\right)$ es el componente parámetros y capta las diferencias de las estructuras de los grupos, es decir, la diferencia en los retornos de las características de ambos grupos. Mientras que el segundo término $\triangle_{X}^{v}=v\left(F_{C}\right)-v\left(F_{0}\right)$ es el componente de composición y se refiere a las diferencias entre las características observables y no observables de ambos grupos. Además tenemos que:

$$
v_{g}=E\left(R I F\left(y_{g} ; v\right) \mid G=g\right)
$$

con $g=0,1 ; \mathrm{y}$

$$
v_{C}=E\left(R I F\left(y_{C} ; v\right) \mid G=1\right) .
$$

Aplicando esperanzas iteradas se tiene que:

$$
m_{g}^{v}(X) \equiv E\left(R I F\left(y_{g} ; v_{g}\right) \mid X, G=g\right)
$$

$\operatorname{para} g=0,1 ; \mathrm{y}$

$$
m_{C}^{v}(X) \equiv E\left(R I F\left(y_{C} ; v_{C}\right) \mid X, G=1\right) .
$$

Con lo cual:

$$
\triangle_{S}^{v}=E\left(m_{1}^{v}(X) \mid G=1\right)-E\left(m_{C}^{v}(X) \mid G=1\right)
$$




$$
\triangle_{X}^{v}=E\left(m_{C}^{v}(X) \mid G=1\right)-E\left(m_{0}^{v}(X) \mid G=0\right) .
$$

Al especificar estas regresiones de RIF en forma lineal:

$$
\begin{gathered}
m_{g}^{v}(X)=X \gamma_{g}^{v} \\
m_{C}^{v}(X)=X \gamma_{C}^{v}
\end{gathered}
$$

el componente parámetros y composición se pueden escribir como:

$$
\begin{aligned}
& \triangle_{S}^{v}=E(X \mid G=1)\left(\gamma_{1}^{v}-\gamma_{C}^{v}\right) \\
& \triangle_{X}^{v}=E(X \mid G=1) \gamma_{C}^{v}-E(X \mid G=0) \gamma_{0}^{v} \\
& =(E(X \mid G=1)-E(X \mid G=0)) \gamma_{0}^{v}+E(X \mid G=1)\left(\gamma_{C}^{v}-\gamma_{0}^{v}\right) \\
& \quad=(E(X \mid G=1)-E(X \mid G=0)) \gamma_{0}^{v}+R^{v}
\end{aligned}
$$

donde $R^{v}$ es un error de aproximación. Se espera que el error sea pequeño cuando el grupo contrafactual es una buena aproximación.

La especificación lineal de las regresiones RIF, siguiendo a Firpo, Fortin y Lemieux (2007, pp. 15), en la práctica resulta una opción válida porque: 1) cualquiera sea la especificación, lineal o no lineal, de todos modos se obtiene un error de aproximación, ya que el procedimiento solo da una aproximación de primer orden para el impacto de "grandes" cambios en la distribución; 2) la especificación lineal no afecta las estimaciones globales de la estructura de la variable de resultado y los efectos de composición que se obtienen mediante el procedimiento de ponderación, es decir, se puede calcular $v_{0}, v_{1}, v_{C}$ independientemente de la especificación; 3) el uso de una especificación lineal tiene la ventaja de proporcionar una interpretación mucho más simple como la de la descomposición Oaxaca-Blinder.

La principal ventaja de este método es que el componente de parámetros (2.7) y el componente composición (2.8) se pueden reescribir en términos de la contribución individual de cada variable $k$ como:

$$
\begin{aligned}
& \triangle_{S}^{v}=\left(\gamma_{1,1}^{v}-\gamma_{C, 1}^{v}\right)+\sum_{k=2}^{K} E\left(X_{k} \mid G=1\right)\left(\gamma_{1, k}^{v}-\gamma_{C, k}^{v}\right), \\
& \triangle_{X}^{v}=\sum_{k=1}^{K}\left(E\left(X_{k} \mid G=1\right)-E\left(X_{k} \mid G=0\right)\right) \gamma_{0, k}^{v}+R^{v}
\end{aligned}
$$

donde el primer término de (2.9) es la diferencia de los interceptos del modelo. Por último, este método de descomposición es path independent. Un método de descomposición es path independent cuando el orden en el que se calculan los diferentes elementos de la descomposición detallada no afecta a los resultados de la descomposición (Fortin et al., 2011). 


\subsubsection{Medida de desigualdad de oportunidades}

Se propone considerar como medida de desigualdad de oportunidades a la suma de las contribuciones de las variables no aceptables $\left(\triangle_{I O}^{v}\right)$. Esta medida provee una aproximación del cambio de la desigualdad de oportunidades entre dos momentos en el tiempo y cómo la misma contribuyó a la desigualdad total en dicho período.

Si se asume que el modelo incluye $J$ variables no aceptables utilizando las ecuaciones (2.9) y (2.10) se tiene que,

$$
\begin{gathered}
\triangle_{I O}^{v}=\triangle_{X_{J}}^{v}+\triangle_{S_{J}}^{v} \\
=\sum_{j=1}^{J}\left(E\left(X_{j} \mid G=1\right)-E\left(X_{j} \mid G=0\right)\right) \gamma_{0, j}^{v} \\
+\sum_{j=1}^{J} E\left(X_{j} \mid G=1\right)\left(\gamma_{1, j}^{v}-\gamma_{c, j}^{v}\right)
\end{gathered}
$$

donde $j \in\{1, \ldots, J\} \subset K$. Si $\triangle_{I O}^{v}$ es positivo entonces la desigualdad de oportunidades medida a través de $v$ ha aumentado entre $t_{0}$ y $t_{1}$ y si es negativo la misma ha disminuido.

Cabe señalar que si bien en la práctica la clasificación entre variables socialmente aceptables y no aceptables no es sencilla porque depende de los juicios de valor de cada sociedad, esta es independiente a la medida aquí propuesta ya que el método de descomposición con el que se trabaja provee un análisis individual de cada variable. De esta manera la clasificación de variables es más flexible ya que se puede conocer el aporte de las variables en forma individual o la contribución en forma conjunta. Se pueden agrupar los efectos de las distintas variables tratando a las variables que no son obvias de las dos maneras: como aceptables y como no aceptables, mostrando así las diferencias de clasificarlas de una forma u otra. Otras ventajas de esta medida es que brinda una interpretación sencilla y la misma se puede extender a cualquier estadístico $v$ que posea una función de influencia (Essama-Nssah et al., 2011, detallan funciones de influencia de diferentes estadísticos).

\subsubsection{Estimación}

En la práctica para la descomposición es necesario estimar $v_{1}, v_{0}, v_{C}$; los vectores de coeficientes $\gamma_{0}, \gamma_{1}, \gamma_{C}$ y las esperanzas de $X$ condicionadas a $G$. Los estadísticos $v_{0}$ y $v_{1}$ se computan a partir de los datos observados $\left(\hat{v}_{g}=v\left(Y_{g}\right), g=0,1\right)$ y $\hat{v}_{C}$ se estima a partir del contrafactual $\left(\hat{v}_{C}=v\left(\hat{Y}_{C}\right)\right)$.

Los coeficientes $\gamma_{0}^{v}, \gamma_{1}^{v}$ y $\gamma_{C}^{v}$ se estiman por MCO. Si se define $I=\{1,2, \ldots, N\}=I_{0} \cup I_{1} \cup I_{C}$ y los conjuntos de subíndices

$$
\begin{aligned}
I_{0} & :=\left\{i \in I / G_{i}=0\right\} \\
I_{1} & :=\left\{i \in I / G_{i}=1\right\} \\
I_{C} & :=\left\{i \in I / G_{i}=C\right\}
\end{aligned}
$$

se tiene que

$$
\hat{\gamma}_{g}^{v}=\left(\sum_{i \in I_{g}} \mathbf{x}_{i} \mathbf{x}_{i}^{\prime}\right)^{-1} \sum_{i \in I_{g}} \mathbf{x}_{i} R \hat{I} F\left(y_{i}, v_{g}\right)
$$


con $g=0,1$ y $\mathbf{x}_{i}$ un vector de $K \times 1 ; \mathrm{y}$

$$
\hat{\gamma}_{C}^{v}=\left(\sum_{i \in I_{C}} \mathbf{x}_{i} \mathbf{x}_{i}^{\prime}\right)^{-1} \sum_{i \in I_{C}} \mathbf{x}_{i} R \hat{I} F\left(y_{i}, v_{C}\right) .
$$

Luego, en las ecuaciones (2.7) y (2.8), se reemplazan los coeficientes estimados en (2.12) y (2.13) y las respectivas medias condicionadas muestrales, obteniendo:

$$
\begin{gathered}
\hat{\triangle}_{S}^{v}=\left(\frac{1}{n_{1}} \sum_{i \in I_{1}} \mathbf{x}_{i}^{\prime}\right)\left(\hat{\gamma}_{1}^{v}-\hat{\gamma}_{C}^{v}\right), \\
\hat{\triangle}_{X}^{v}=\left(\frac{1}{n_{1}} \sum_{i \in I_{1}} \mathbf{x}_{i}^{\prime}-\frac{1}{n_{0}} \sum_{i \in I_{0}} \mathbf{x}_{i}^{\prime}\right) \hat{\gamma}_{0}^{v}+\hat{R}^{v},
\end{gathered}
$$

donde $\hat{R}^{v}=\left(\frac{1}{n_{1}} \sum_{i \in I_{1}} \mathbf{x}_{i}^{\prime}\right)\left(\hat{\gamma}_{1}^{v}-\hat{\gamma}_{C}^{v}\right)$ es una estimación del error de aproximación.

La regresión de RIF para el análisis de desigualdad de oportunidades, $m^{v}(X)=X \gamma^{v}=$ $\left[\begin{array}{lll}1 & e^{\prime} & \mathbf{x}^{\prime}\end{array}\right]\left[\begin{array}{c}\gamma_{0}^{v} \\ \gamma_{e}^{v} \\ \gamma_{\mathbf{x}}^{v}\end{array}\right]$, presenta la siguiente forma:

$$
R I F_{i}(v)=\gamma_{0}^{v}+\mathbf{e}_{i}^{\prime} \gamma_{e}^{v}+\mathbf{x}_{i}^{\prime} \gamma_{\mathbf{x}}^{v}+u_{i}
$$

donde $R I F(v)$ es la transformación de la variable desempeño educativo $Y$ a través de la función de influencia recentrada del funcional $v, \mathbf{e}_{i}$ es un vector $M_{2} \times 1$ de variables aceptables, $\mathbf{x}_{i}$ es un vector $J \times 1$ de variables no aceptables, $\gamma_{e}^{v}$ es un vector de coeficientes $M_{2} \times 1, \gamma_{\mathbf{x}}^{v}$ es un vector de coeficientes $J \times 1$ y $u_{i}$ es un término de error aleatorio.

La ecuación (2.14) se estima para cada grupo $G=g$, en este caso el grupo $G=0$ corresponde al año base $\left(t_{0}=0\right), G=1$ al año de referencia $\left(t_{1}=1\right)$ y $G=C$ al grupo contrafactual. Las estimaciones se realizan a partir de la muestra ponderada. En el caso del grupo contrafactual se ajusta el ponderador del grupo 0 a partir de una función de reponderación $\omega_{C}$ que tiene en cuenta la probabilidad estimada de pertenecer al grupo 1, $\hat{p}, \mathrm{y}$ la probabilidad estimada de pertenecer al grupo 1 dado $X, \hat{p}(X)$. La función estimada es $\hat{w}_{C}(G, X)=\frac{1-G}{\hat{p}} \frac{\hat{p}(X)}{(1-\hat{p}(X))}$.

Respecto al término no observable $u$, se supone que para todo $x$ en $X$ dado $X=x, u$ es independiente de $G$. La idea detrás es que la distribución de los factores explicativos no observados del desempeño educativo no debería depender de los grupos elegidos, una vez que se condiciona por $X$. En este trabajo donde $G$ corresponde a dos momentos de tiempo, este último supuesto es plausible. Los periodos considerados para este estudio no son de largo plazo, por lo que es esperable que controlando por características del estudiante no hayan cambios significativos en la educación del país entre los años 2009 y 2012 o 2006 y 2012 que hagan que los factores no observables $u$ puedan haber cambiado significativamente en el periodo considerado. En cuanto al periodo 2000 y 2012, donde si bien sucedieron algunos acontecimientos como la crisis económica del 2001 y una nueva legislación educativa (en el año 2006 se cambia la Ley Federal de Educación por la Ley de Educación Nacional) que podrían hacer que los no observables estuvieran relacionados con el periodo de tiempo, es 
posible esperar que una vez que se controla por distintas variables de los hogares, alumnos y colegios, $u$ sea independiente del periodo considerado.

Por otro lado, hasta aquí no se ha mencionado la posibilidad de problemas de endogeneidad en las estimaciones. El método de estimación supone que los regresores son variables exógenas, sin embargo es posible que algunas de ellas sean endógenas. Si bien esto podría ser una limitación, podría no ser un problema si el sesgo es homogéneo en todas las partes de la distribución y el objetivo es analizar los efectos de las variables sobre medidas distributivas. Por ejemplo, si el sesgo (hacia arriba o hacia abajo) es el mismo en todos los cuantiles de la distribución y el análisis que se hace corresponde a las brechas de cuantiles, en principio, la subestimación o sobrestimación no estaría afectando la validez de las conclusiones de los resultados. Es decir, si el sesgo es el mismo en todos los cuantiles, al calcular las brechas de cuantiles se compensaría sin traer mayores complicaciones. En cambio, si existen heterogeneidades no observadas (el sesgo es diferente en los distintos puntos de la distribución), la endogeneidad sí podría conducir a conclusiones desacertadas. Si bien cierta literatura reciente se ha enfocado en esta línea de investigación todavía los estudios se están desarrollando (ver Frölich y Melly, 2013; Firpo y Pinto, 2016; Arellano y Bonhomme, 2017; Melly y Wuthrich, 2017).

\subsection{Variables y datos}

La fuente de información es la base de datos de las pruebas PISA de los años 2000, 2006, 2009 y $2012 .{ }^{4} \mathrm{El}$ análisis se enfoca en el desempeño educativo de los alumnos medido a través de los puntajes de la prueba de matemáticas. ${ }^{5}$ También se realizaron estimaciones para las competencias de lectura y ciencia, encontrando en general resultados similares a matemáticas. En las siguientes secciones solamente se presentan los resultados de esta última competencia.

Se estiman tres modelos considerando distintos conjuntos de variables aceptables y no aceptables. En el modelo 1 las variables socialmente no aceptables tenidas en cuenta para el análisis son el género, la educación y la ocupación de los padres, la composición del hogar (si ambos padres se encuentran en el hogar o si es monoparental), el estatus migratorio, los recursos educativos (computadora, internet y libros en el hogar) y la localización geográfica. En cuanto a las variables aceptables se consideran la edad del alumno y el año escolar cursa. ${ }^{6}$ El modelo 2 incorpora además de las variables anteriores, el contexto socioeconómico del colegio como variable no aceptable. Para ello se considera el nivel educativo promedio de los padres del colegio. El tercer modelo incluye además de las variables anteriores, una variable aceptable relacionada con el esfuerzo del alumno que consiste en el tiempo que dedica el alumno a estudiar matemáticas fuera del colegio según su propio reporte.

$\mathrm{Al}$ igual que en el Capítulo 1, se considera al género como un factor no aceptable. En general en la mayoría de los países los varones presentan puntajes más altos en matemáticas que las mujeres. Sin embargo, aún siendo tan usual este resultado educativo, las brechas de género son motivo de preocupación para la sociedad y los hacedores de políticas, tal es así que en la Ley de Educación Nacional 26.206 del país se postula que no es admisible ningún tipo de discriminación por género (art. 11, inciso f y art. 84). Según la legislación tampoco son aceptables las brechas regionales o las brechas por ser migrante (art. 11, inciso a, art.

\footnotetext{
${ }^{4}$ Si bien, en el año 2003 se llevaron a cabo las pruebas PISA, en dicha ocasión Argentina no participó del programa y por eso no se cuenta con información para dicho año.

${ }^{5}$ Para las estimaciones se tuvieron en cuenta las particularidades de PISA de esta variable, para más detalles ver Anexo 1 del Capítulo 1 así como OCDE (2009, 2005).

${ }^{6}$ La selección de las variables mencionadas aquí ya han sido explicadas en forma extensiva en el primer capítulo de esta tesis (ver pág. 10).
} 
84 y art. 141). Asimismo, siguiendo la legislación el desempeño educativo del alumno no debería depender de su origen social y, por lo tanto, el contexto familiar medido a través de la educación y la ocupación de los padres, la composición y los recursos educativos del hogar no deberían ser condicionante de los logros educativos. Estos factores son considerados socialmente no aceptables. También se considera no aceptable el contexto socioeconómico del colegio. Por otro lado, existen diferencias derivadas de la edad de los alumnos que como se explicó en el capítulo anterior, pueden ser socialmente aceptables así como las derivadas del año escolar que estén cursando y el tiempo de estudio que dedican a matemáticas. La Tabla 2.1 presenta las distintas variables regresoras que se utilizan en cada modelo.

Tabla 2.1: Definición de variables para las descomposiciones.

\begin{tabular}{|c|c|}
\hline Variable & Definición \\
\hline \multicolumn{2}{|l|}{ Aceptables } \\
\hline Edad & edad $=$ edad del joven en años y meses \\
\hline Tiempo dedicado & tiempomath= 1 si dedica más de dos horas por semana fuera del colegio a estudiar matemáticas \\
\hline Año escolar & ano10 = 1 si asiste al año escolar que le corresponde según su edad \\
\hline \multicolumn{2}{|l|}{ No aceptables } \\
\hline Género & hombre $=1$ si es hombre \\
\hline Educación de los padres & educpadres = máxima educación de los padres en años \\
\hline Estructura familiar & ambospadres $=1$ si ambos padres conforman el hogar \\
\hline \multirow[t]{3}{*}{ Ocupación de los padres } & $\begin{array}{l}\text { ocupbaja = } 1 \text { la máxima ocupación de los padres es como expertos agrícolas y/o de la pesca, } \\
\text { trabajador u ocupado en ocupaciones elementales }\end{array}$ \\
\hline & ocupmedia = 1 la máxima ocupación de los padres es como trabajador de servicios, artesanía \\
\hline & $\begin{array}{l}\text { ocupalta }=1 \text { la máxima ocupación de los padres es legisladores, altos funcionarios y } \\
\text { profesionales, técnicos y empleados }\end{array}$ \\
\hline Estatus migratorio & migra $=1$ si nació en otro país y/o sus padres nacieron en otro paíss \\
\hline & idiomahogar $=1$ si la lengua hablada en el hogar es distinta al idioma de la prueba \\
\hline Localización geográfica & $\begin{array}{l}\text { escpueblopeq }=1 \text { escuela localizada en un pueblo pequeño de menos de } 15.000 \text { hab. } \\
\text { escpueblomed }=1 \text { escuela localizada en un pueblo entre } 15.000 \text { y } 100.000 \text { hab. } \\
\text { escciudad }=1 \text { escuela localizada en una ciudad de más de } 100.000 \text { hab. }\end{array}$ \\
\hline Recursos educativos & $\begin{array}{l}\text { libros }=1 \text { si posee más de } 100 \text { libros en el hogar } \\
\text { computadora }=1 \text { si posee computadora en el hogar } \\
\text { internet }=1 \text { si posee internet en el hogar }\end{array}$ \\
\hline Contexto socioeconómico & contextosocioec $=$ educación promedio de los padres del colegio en años \\
\hline
\end{tabular}

La muestra de PISA de Argentina contiene 4289 observaciones en 2012 y 1641 en 2000. En el modelo 3 donde se incorpora el tiempo dedicado a matemáticas se trabaja con una submuestra. La variable tiempo dedicado a matemáticas presenta varios datos faltantes, por lo que la cantidad de observaciones de la muestra se reduce. La muestra del año 2012 pasa de tener 4289 observaciones a 2781 y la muestra del año 2000 se reduce a 1573 .

El puntaje promedio de matemáticas de los estudiantes de la muestra principal es de 398, 391, 400 y 402 en 2000, 2006, 2009 y 2012, respectivamente. Mientras que para la submuestra en dichos años ascienden a 403, 396, 418 y 405. Como se puede observar en el Gráfico 2.1 la media de los estudiantes solo ha aumentado levemente entre el 2000 y 2012. Más aún si tenemos en cuenta las respectivas desviaciones estándar este aumento no ha sido estadísticamente significativo como lo muestran los intervalos de confianza del $95 \%$. 


\section{Gráfico 2.1: Evolución de la media de los puntajes de matemática.}

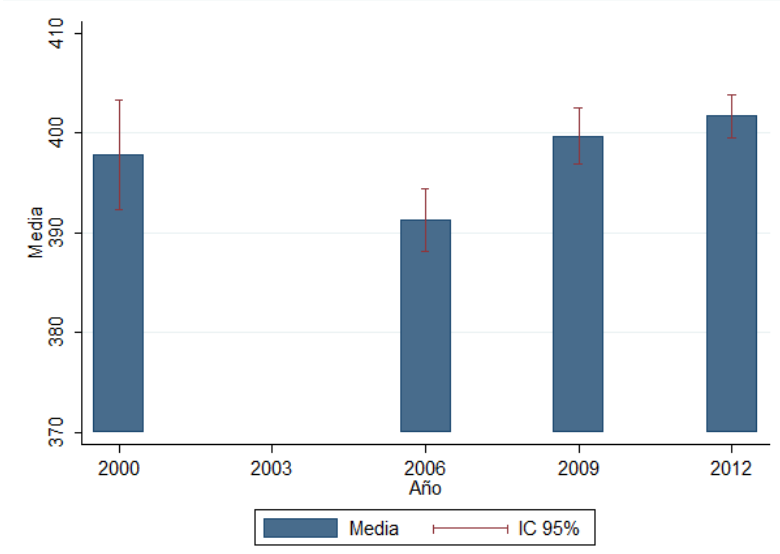

Nota: IC $95 \%$ significa intervalos de confianza del $95 \%$ Fuente: Elaboración propia en base a datos PISA.

Al analizar la evolución de la desigualdad total de los puntajes de matemáticas en los 2000s, se encuentra que la varianza de los puntajes ha disminuido alrededor de la mitad (varió de 12282 a 5233 como se puede verificar en la Tabla A2.2 del Apéndice 2). Asimismo, el coeficiente de Gini, la brecha entre el cuantil 50 y 10 y la brecha entre el cuantil 90 y 50 presentan una disminución entre los años 2000 y $2012 .^{7}$ En el caso de las brechas entre cuantiles, la caída más fuerte se observa en la parte izquierda de la distribución. El Gráfico 2.2, muestra que la brecha entre el cuantil 50 y 10 ha disminuido a una tasa mayor que la brecha de los cuantiles 90 y 50 (ver más detalle en la Tabla A2.2 del Apéndice 2).

Gráfico 2.2: Evolución de la desigualdad del desempeño educativo.
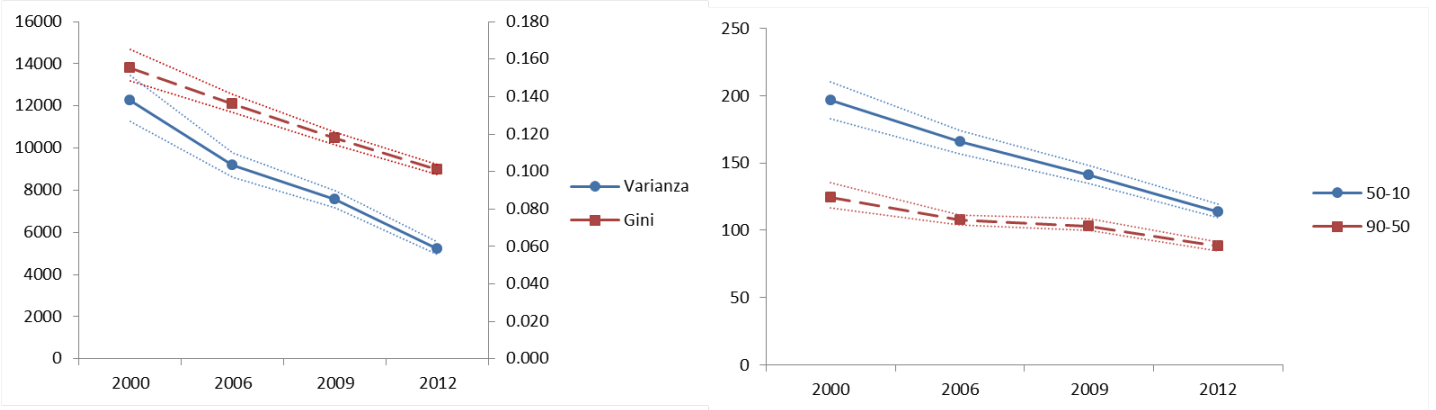

Nota: Líneas punteadas corresponden a intervalos de confianza del $95 \%$ con bootstrap (200 repeticiones) Fuente: Elaboración propia en base a datos PISA.

El resto de las estadísticas descriptivas de las variables de los años 2000, 2006, 2009 y 2012 se presentan en la Tabla A2.1 del Apéndice 2. La parte 1 de la tabla arroja las estadísticas descriptivas de la muestra total mientras que la parte 2 presenta las estadísticas de la submuestra utilizada en el modelo 3. Las mismas indican que la educación máxima de los padres, en promedio, ronda en los 12 años y medio de educación tanto en la primer muestra como en la submuestra. Específicamente, para el año 2000 no se cuenta con la cantidad de años de educación de los padres sino que se cuenta con información del nivel educativo más

\footnotetext{
${ }^{7}$ La brecha de cuantiles se refiere a la diferencia entre el promedio de puntajes del cuantil $q_{\tau}$ y $q_{\tau^{\prime}}$ con $\tau, \tau^{\prime}=10,50,90$.
} 
alto alcanzado. En este caso se incluyen en los modelos las variables dummies seceducpadres y supeducpadres (la categoría omitida es educación primaria o sin educación). La primera toma valor 1 si el nivel máximo educativo alcanzado por alguno de los padres del alumno es secundario completo o incompleto y 0 lo contrario. La segunda toma valor 1 si alguno de los padres tiene educación superior (pos-secundaria). En el año 2000 el $53 \%$ de los alumnos de la muestra tienen padres cuyo máximo nivel educativo alcanzado es educación superior y el $46 \%$ secundario. En cambio en el 2012 estos porcentajes descienden a $49 \%$ y $35 \%$, respectivamente.

La distribución de alumnos según el nivel de ocupación de los padres no ha cambiado a través de los años. Tanto en 2000 como 2012 la mayoría de los padres de los estudiantes de la muestra se encuentran ocupados en puestos laborales altos o medios.

En general, se observa que el porcentaje de hombres y mujeres en ambas muestras es bastante similar. La mayoría de los estudiantes (más del $75 \%$ ) viven con ambos padres en el hogar. El porcentaje de estudiantes que hablan en su hogar un idioma diferente al del examen es muy bajo, al igual que el porcentaje de estudiantes nacidos en otro país o cuyos padres provienen de otro país. Ambas muestras en 2000 y 2012 presentan una mayor concentración de escuelas en pueblos medianos o ciudades. El porcentaje de estudiantes que cursan el año educativo que les corresponde según su edad ha ido disminuyendo durante los años. Como proxy del contexto socioeconómico del colegio se utiliza el porcentaje de padres del colegio con educación superior, en promedio este porcentaje en 2000 es $53 \%$ con un desvío estándar de 0.28 y en 2012 es $49 \%$ con un desvío estándar de 0.22 .

En cuanto a la edad de los alumnos, la misma es, en promedio, levemente superior a los 15 años y medio. En 2000, el $74 \%$ de los alumnos cursan el grado que deberían cursar según su edad mientras que en 2012 este porcentaje disminuye 10 puntos porcentuales pasando a ser el $65 \%$. En la submuestra se encuentra algo similar. A su vez el porcentaje de los alumnos que dedican tiempo a estudiar fuera del colegio ha disminuido en los últimos años. Mientras que en el año 2000 el $58 \%$ de los alumnos estudiaban fuera del colegio, en el año 2012 dicho porcentaje es el $15 \%$.

Finalmente, en 2012 PISA incluyó en el cuestionario algunas preguntas subjetivas sobre el desempeño en matemáticas. En particular, se pregunta si el alumno cree que en matemáticas: puede tener éxito si se esfuerza lo suficiente, que le vaya bien solo depende de él, que si lo desea puede tener un buen desempeño. Si bien, como se mencionó en el Capítulo 1, la distribución de resultados de los puntajes de las pruebas puede ser reflejo de factores socialmente aceptables como las habilidades innatas, tiempo de estudio así como de otras variables de control del alumno; la Tabla 2.2, que reporta los resultados de estas preguntas, muestra que más de la mitad de los alumnos contestan que su desempeño no depende solamente de ellos, ni del esfuerzo que realizan o de su predisposición. Esto sugiere que existen otras variables que también determinan los puntajes de matemáticas de los alumnos más allá del control que ellos puedan ejercer. En particular, si algunas de estas variables se asocian a factores no aceptables entonces se estaría ante una situación de significativa desigualdad de oportunidades. De ahí la importancia de analizar el efecto individual de cada variable mencionada en esta sección. 
Tabla 2.2: Autorespuesta sobre el desempeño en matemáticas.

\begin{tabular}{lcccccc}
\hline $\begin{array}{c}\text { Tipo de control } \\
\text { del alumno }\end{array}$ & \multicolumn{2}{c}{$\begin{array}{c}\text { Se puede tener } \\
\text { éxito en mat. con } \\
\text { el suficiente esfuerzo. }\end{array}$} & $\begin{array}{c}\text { Que me vaya bien } \\
\text { en mat. depende } \\
\text { solamente de mi. }\end{array}$ & $\begin{array}{c}\text { Si quiero puedo } \\
\text { tener una buena } \\
\text { performance en mat. }\end{array}$ \\
\hline Respuesta & $\mathrm{Si}$ & No o NS-NC & $\mathrm{Si}$ & No o NS-NC & $\mathrm{Si}$ & No o NS-NC \\
\hline Total & $47 \%$ & $53 \%$ & $45 \%$ & $55 \%$ & $45 \%$ & $55 \%$ \\
Hombres & $46 \%$ & $54 \%$ & $45 \%$ & $55 \%$ & $45 \%$ & $55 \%$ \\
Mujeres & $48 \%$ & $52 \%$ & $45 \%$ & $55 \%$ & $44 \%$ & $56 \%$ \\
\hline
\end{tabular}

Fuente: Elaboración propia en base a datos PISA.

\subsection{Resultados}

Antes de pasar a los resultados de las descomposiciones es conveniente señalar que todo cambio distributivo se lo puede concebir como el resultado de la interacción de los diferentes grupos de variables. Por un lado, las variables aceptables y no aceptables pueden contribuir a la desigualdad en la misma dirección aumentando o disminuyéndola. O bien, ambos grupos pueden hacerlo en forma opuesta. Por otro lado, un grupo de variables, por ejemplo las no aceptables, podrían no contribuir a cambios en la desigualdad mientras que el otro grupo de variables, las aceptables, sí o viceversa. En particular, los escenarios más relevantes son aquellos en los cuales las variables no aceptables contribuyen a aumentar la desigualdad independientemente del cambio final de la desigualdad total. Es en estos escenarios donde se produce un aumento de la desigualdad de oportunidades.

En este caso la disminución de la desigualdad de los puntajes de matemáticas observada en la sección anterior en el Gráfico 2.2 es compatible con distintos escenarios de desigualdad de oportunidades. A pesar de la disminución total, la desigualdad de oportunidades podría haber aumentado si las variables no aceptables contribuyeron a aumentar la desigualdad en el tiempo. O podría haber disminuido si las variables no aceptables contribuyeron a la caída de la desigualdad total. En esta sección se indaga cuál ha sido el rol de las variables no aceptables en la caída de la desigualdad total entre los años 2000 y 2012.

\subsubsection{Descomposición de desigualdad de oportunidades educati- vas más allá de la media.}

\section{Regresiones de RIF}

En la Tabla 2.3 se presentan las estimaciones del modelo 1 para el año 2012. Este modelo incluye en su especificación las variables edad, año escolar, género, educación y ocupación de los padres, estructura del hogar, estatus migratorio, recursos educativos y localización geográfica. La primer columna de la tabla contiene las estimaciones clásicas por MCO, en las siguientes se exponen los resultados de las regresiones de RIF de los cuantiles no condicionados 10, 50 y 90 y las últimas dos columnas corresponden a las regresiones de RIF de la varianza y el coeficiente de Gini. Las estimaciones de los modelos 2 y 3 se encuentran en la Tabla A2.3 del Apéndice 2. El modelo 2 incorpora además de las variables del modelo 1, el contexto socioeconómico del colegio y el modelo 3 incluye también el tiempo que dedica el alumno a estudiar matemáticas fuera del colegio. 
Tabla 2.3: Estimaciones del desempeño educativo por MCO clásico y RIF.

\begin{tabular}{|c|c|c|c|c|c|c|}
\hline & & \multicolumn{5}{|c|}{ Modelo 1} \\
\hline Variables & MCO clásico & 0.10 & 0.50 & 0.90 & Varianza & Gini \\
\hline \multirow[t]{2}{*}{ hombre } & 21.52 & 20.45 & 23.73 & 18.02 & 235.65 & -0.004 \\
\hline & $(2.19)^{* * *}$ & $(6.67)^{* * *}$ & $(3.89)^{* * *}$ & $(5.83)^{* * *}$ & $(315.48)$ & $(0.003)$ \\
\hline \multirow[t]{2}{*}{ ambospadres } & -0.64 & -2.73 & -0.99 & -0.04 & -88.71 & 0.000 \\
\hline & $(2.75)$ & $(6.03)$ & $(4.36)$ & $(6.04)$ & $(385.69)$ & $(0.004)$ \\
\hline \multirow[t]{2}{*}{ educpadres } & 2.09 & 1.58 & 1.89 & 2.09 & 0.24 & -0.001 \\
\hline & $(0.29)^{* * *}$ & $(0.88)^{*}$ & $(0.61)^{* * *}$ & $(0.67)^{* * *}$ & $(40.01)$ & $(0.000)$ \\
\hline \multirow[t]{2}{*}{ ocupbaja } & -26.77 & -30.40 & -28.58 & -19.43 & 282.98 & 0.010 \\
\hline & $(3.23)^{* * *}$ & $(11.29)^{* * *}$ & $(6.61)^{* * *}$ & $(8.46)^{* *}$ & $(542.64)$ & $(0.005)^{*}$ \\
\hline \multirow[t]{2}{*}{ ocupmedia } & -21.66 & -15.07 & -24.59 & -20.29 & -459.49 & 0.001 \\
\hline & $(2.58)^{* * *}$ & $(6.97)^{* *}$ & $(5.17)^{* * *}$ & $(7.25)^{* * *}$ & $(308.55)$ & $(0.003)$ \\
\hline \multirow[t]{2}{*}{ idiomahogar } & -11.36 & -18.76 & -12.01 & -4.48 & 1043.90 & 0.012 \\
\hline & $(10.48)$ & $(35.56)$ & $(15.17)$ & $(12.25)$ & $(1521.83)$ & $(0.017)$ \\
\hline \multirow[t]{2}{*}{ migra } & -10.35 & -12.08 & -11.95 & -5.51 & 648.87 & 0.008 \\
\hline & $(3.50)^{* * *}$ & (14.33) & $(5.83)^{* *}$ & $(6.51)$ & $(673$ & $(0.1$ \\
\hline \multirow[t]{2}{*}{ escpueblopeq } & -11.48 & -4.49 & -15.82 & -9.81 & -470.40 & -0.001 \\
\hline & $(3.05)^{* * *}$ & $(7.32)$ & $(4.41)^{* * *}$ & $(8.75)$ & $(394.55)$ & $(0.004)$ \\
\hline \multirow[t]{2}{*}{ escpueblomed } & -11.27 & -7.53 & -14.11 & -10.46 & -264.90 & 0.000 \\
\hline & $(2.56)^{* * *}$ & $(4.88)$ & $(4.47)^{* * *}$ & $(6.13)^{*}$ & $(392.93)$ & $(0.004)$ \\
\hline \multirow[t]{2}{*}{ libros } & 24.72 & 11.25 & 23.21 & 35.29 & 1805.78 & 0.011 \\
\hline & $(3.00)^{* * *}$ & $(4.96)^{* *}$ & $(4.76)^{* * *}$ & $(8.55)^{* * *}$ & $(429.47)^{* * *}$ & $(0.004)^{* * *}$ \\
\hline \multirow[t]{2}{*}{ computadora } & 9.42 & 6.33 & 11.57 & 7.05 & -17.62 & -0.003 \\
\hline & $(3.60)^{* * *}$ & (11.18) & $(5.86)^{* *}$ & $(6.18)$ & $(490.66)$ & $(0.005)$ \\
\hline \multirow[t]{2}{*}{ internet } & 10.85 & 14.68 & 9.51 & 5.15 & -514.90 & -0.007 \\
\hline & $(3.15)^{* * *}$ & $(8.29)^{*}$ & $(5.49)^{*}$ & $(5.67)$ & $(465.12)$ & $(0.005)$ \\
\hline \multirow[t]{2}{*}{ edad } & 6.71 & 8.52 & 2.92 & 14.19 & 152.29 & 0.000 \\
\hline & $(3.63)^{* *}$ & (10.61) & $(5.85)$ & $(10.58)$ & $(596.40)$ & $(0.007)$ \\
\hline \multirow[t]{2}{*}{ año escolar } & 49.75 & 62.84 & 49.97 & 30.24 & -1412.33 & -0.027 \\
\hline & $(2.43)^{* * *}$ & $(14.69)^{* * *}$ & $(5.51)^{* * *}$ & $(5.65)^{* * *}$ & $(318.16)^{* * *}$ & $(0.003)^{* * *}$ \\
\hline \multirow[t]{2}{*}{ constante } & 230.88 & 105.93 & 294.17 & 223.68 & 4265.39 & 0.133 \\
\hline & $(57.50)^{* * *}$ & $(175.48)$ & $(93.19)^{* * *}$ & $(170.69)$ & $(9603.57)$ & $(0.106)$ \\
\hline \multirow{6}{*}{\multicolumn{7}{|c|}{$\begin{array}{l}\text { Nota: La variable dependiente es el puntaje de la prueba de matemáticas. El modelo } 1 \text { incluye } \\
\text { como variables explicativas socialmente no aceptables al género, educación y ocupación de los } \\
\text { padres, estructura del hogar, estatus migratorio, recursos educativos y localización geográfica e } \\
\text { incluye como aceptables las variables edad del estudiante y año escolar. Errores estándar con } \\
\text { bootstrap (200 repeticiones) entre paréntesis. }{ }^{*} \text { significativo al } 10 \% ;{ }^{* *} \text { significativo al } 5 \% ; \\
* * * \text { significativo al } 1 \% \text {. }\end{array}$}} \\
\hline & & & & & & \\
\hline & & & & & & \\
\hline & & & & & & \\
\hline & & & & & & \\
\hline & & & & & & \\
\hline \multicolumn{7}{|c|}{ Fuente: Elaboración propia en base a datos PISA. } \\
\hline
\end{tabular}

A partir de las estimaciones de los modelos se advierte que los efectos son heterogéneos a través de los diferentes cuantiles. Para analizar algunas de las heterogeneidades encontradas, se presentan gráficamente los coeficientes estimados de las regresiones del cuantil 10 al cuantil 90 del modelo 1. La Figura 1 del Gráfico 2.3 muestra que el efecto positivo del género no es monótono, primero aumenta hasta el cuantil 40 y luego disminuye presentando una forma de U invertida. Esto sugiere que si bien a los varones les va mejor en matemáticas el efecto es menor en los primeros y últimos cuantiles. De esta manera, el género aumenta la brecha entre los primeros cuantiles y los cuantiles centrales (pendiente positiva) y disminuye la brecha con los últimos cuantiles (pendiente negativa). En particular, el género no tiende a aumentar la desigualdad global ya que el efecto es mayor en el centro de la distribución. El efecto sobre la brecha entre los cuantiles del medio con los cuantiles más altos es mayor que el efecto sobre la brecha entre los cuantiles centrales y los cuantiles más bajos. Más aún, si se tienen en cuenta medidas resumen de la desigualdad más globales como el Gini, el coeficiente de la regresión de RIF de esta variable, que se muestra en la Tabla 2.3 resulta negativo y en el caso de la varianza si bien es positivo es pequeño. Además en ambos casos dichos coeficientes no son estadísticamente significativos. 
Gráfico 2.3: Coeficientes estimados de las regresiones de cuantiles no condicionados del modelo 1.
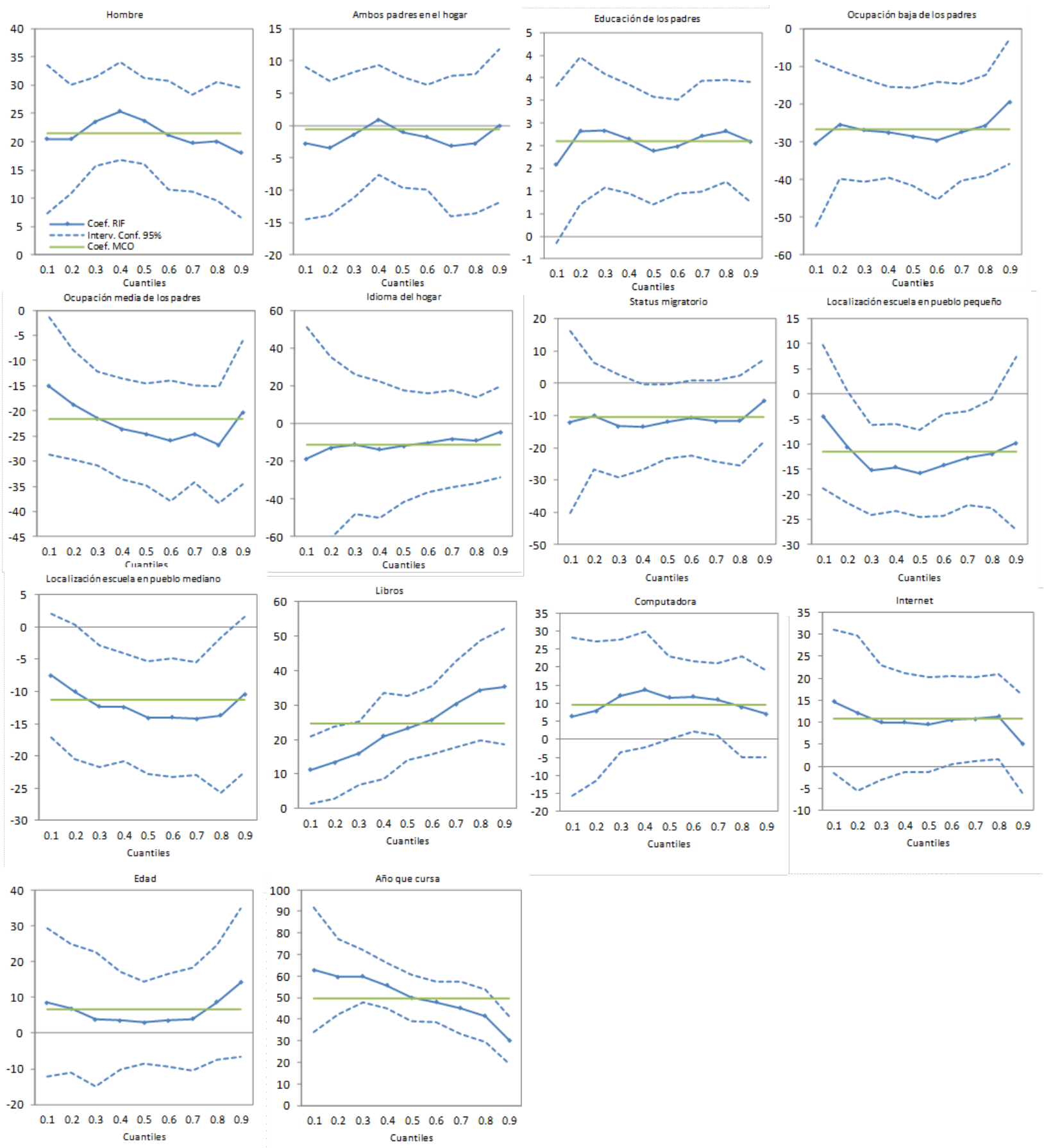

Nota: El modelo 1 incluye como variables explicativas socialmente no aceptables al género, educación y ocupación de los padres, estructura del hogar, estatus migratorio, recursos educativos y localización geográfica e incluye como aceptables las variables edad del estudiante y año escolar. Intervalos de confianza al $95 \%$.

Fuente: Elaboración propia en base a datos PISA.

Otro factor que presenta un patrón similar es la variable computadora en el hogar. El efecto positivo de esta variable en los distintos cuantiles no es monótono mostrando gráficamente una forma de U invertida. Como el mayor valor de los coeficientes se observa alrededor del cuantil 40, su impacto global sobre la desigualdad no es estadísticamente significativo.

La variable educación de los padres presenta coeficientes positivos para cada cuantil similar al coeficiente de MCO clásico. En forma global, no presenta un impacto estadísticamente 
significativo sobre el Gini o la varianza. Por otro lado, como era de esperar la ocupación de los padres arroja coeficientes negativos en cada cuantil (la variable dummy omitida es la correspondiente a ocupación alta). Los efectos negativos en valor absoluto son mayores en los cuantiles centrales, incrementando la desigualdad total.

En el caso de los libros se encuentra un efecto positivo monótono creciente. Esto sugiere que el impacto de aumentar la proporción de alumnos cuyos hogares presentan una mayor disposición a la lectura es positivo y es mayor en los cuantiles más altos que en los cuantiles más bajos. Es decir, no solo aumenta el nivel de los puntajes de matemáticas sino que aumenta la dispersión de los mismos. Es importante señalar nuevamente que la cantidad de libros que hay en un hogar refleja la inclinación de la familia a leer, lo que es una medida proxy de la predisposición de la familia hacia la educación. Si se tienen en cuenta medidas más globales de desigualdad se observa que los libros en el hogar tienen un impacto positivo y significativo en el Gini y en la varianza. Es decir, que la proporción de alumnos cuyos hogares poseen una predisposición favorable hacia la educación se puede asociar con una mayor desigualdad total de los puntajes de matemáticas.

Otros factores cuyos efectos son mayores a medida que se avanzan hacia los cuantiles más altos (aunque más leves) son el estatus migratorio y el idioma del hogar. En este caso, los coeficientes son negativos, sugiriendo que a medida que se avanza a los cuantiles más altos el efecto de ser extranjero es cada vez menor. Aumentando así la brecha de puntajes entre los cuantiles más altos y más bajos.

En cuanto la variable año escolar es esperable que, en principio, tenga un efecto positivo en el desempeño educativo, sin embargo este efecto puede no ser obvio ya que podría depender de la actitud de los sistemas educativos respecto a la repitencia. Si se tienen en cuenta dos situaciones: (1) sistemas educativos donde la promoción de los alumnos depende de las competencias de los mismos y (2) sistemas educativos en donde la probabilidad de que un alumno repita el curso es baja independientemente de sus competencias, en la primera situación el año escolar es una buena señalización de las competencias de los alumnos y se esperaría que el efecto de esta variable sea positivo. No obstante, su impacto en la desigualdad dependerá de la intensidad del efecto de esta variable en las distintas partes de la distribución. En la segunda situación donde el año escolar no es una buena señalización, los sistemas educativos tienen que adaptarse a cursos heterogéneos de alumnos y hacer frente a la variedad de capacidades e intereses de los estudiantes, lo que puede derivar en un efecto menor en el desempeño educativo y a una mayor desigualdad. En Argentina, según datos de la DINIECE (2016), la tasa de repitencia de la educación primaria en 2012 es alrededor de $2.8 \%$ mientras que la tasa de repitencia de los primeros tres años de la educación secundaria es de $12 \%$ y en los últimos años de $6 \%$, por lo que esta tasa es alta en el nivel medio. Las altas tasas de repitencia en el nivel medio podrían llevar a que el efecto de la variable año escolar sea positivo en el desempeño educativo de los alumnos. Las estimaciones de los coeficientes de esta variable son positivos en los diferentes cuantiles, indicando que cursar el año escolar de acuerdo a la edad se relaciona con un efecto positivo en el desempeño educativo. Este efecto es monótono decreciente en los diferentes cuantiles. El mismo es mayor en los cuantiles más bajos y es menor en los cuantiles más altos, cerrando la brecha entre los cuantiles. También se puede observar que el coeficiente de esta variable en la regresión de la varianza y Gini toma valores negativos, evidenciando nuevamente lo encontrado para el caso de los cuantiles. Es decir, que esta variable no se asociaría con un aumento de la desigualdad global ya que el efecto es mayor en los cuantiles más bajos.

En el caso de la localización de las escuelas se halla un coeficiente negativo presentando una gráfica en forma de U. Es decir, los alumnos que asisten a escuelas que se encuentran en pueblos pequeños o medianos muestran un menor desempeño que los alumnos de zonas 
urbanas. Por otro lado, dichos efectos negativos son menores en los cuantiles más bajos y en los más altos y son mayores en los cuantiles centrales. Esto reduce la brecha entre los cuantiles más bajos y los cuantiles centrales, pero aumenta la brecha con los cuantiles más altos. No obstante, los efectos sobre el coeficiente de Gini y la varianza no son estadísticamente significativos.

\section{Descomposición de RIF}

En la Tabla 2.4 se presentan los resultados de la descomposición para el período 20002012, que abarca la primer década de los 2000s. La descomposición de RIF se realiza para el coeficiente de Gini, la varianza y las brechas entre los cuantiles 90 y 10, 50 y 10 y 90 y 50 utilizando las estimaciones del modelo 1. En el Apéndice 2 se presentan los resultados correspondientes al modelo 2 y al modelo 3.

Entre los años 2000 y 2012, la desigualdad de los puntajes de matemáticas disminuyó en el país. El coeficiente de Gini disminuyó 0.059 puntos, la varianza 7669.90 puntos, la brecha 90-10 94.46 puntos, la brecha 50-10 58.88 puntos y la brecha 90-50 35.58 puntos. Sin embargo, el componente composición arroja valores positivos, sugiriendo que las características de los alumnos han contribuido al aumento de la desigualdad en este período. No obstante, este efecto es menor respecto al componente parámetros. Este último muestra valores negativos en todos los casos favoreciendo a la disminución de la desigualdad en dicho período y es en valor absoluto más grande que el efecto composición, dirigiendo así el cambio global.

En la primera parte de la Tabla 2.4 se presenta la descomposición detallada del componente composición mientras que en la segunda parte se presenta la descomposición del componente de parámetros. A partir del análisis individual, se encuentra que los factores no aceptables que más contribuyeron al efecto composición aumentando la desigualdad son los recursos educativos. Este efecto es mayor en el lado izquierdo de la distribución (brecha cuantiles 50-10) que en el lado derecho (brecha 90-50). En el caso de las variables aceptables el año cursado tiene un efecto positivo contribuyendo al aumento de la desigualdad en dicho período.

La mayor parte del componente parámetros se atribuye al término residual (término de constantes de la ecuación (2.9)) y la otra gran parte es atribuida a las variables aceptables. En cuanto a las demás variables, se observa que los retornos a los recursos han contribuido a aumentar la desigualdad así como los de la localización, en particular han contribuido a incrementar la brecha 90-50 (lado derecho de la distribución). Los retornos de la ocupación y educación de los padres solo contribuyeron a aumentar la brecha de cuantiles 50-10.

Por último, el error de ponderación que arroja cada una de las estimaciones es bajo, sugiriendo una buena aproximación dado que el mismo representa una pequeña proporción del

cambio total. Asimismo el error de especificación, $R^{v}$, en general también es relativamente modesto. 
Tabla 2.4: Descomposición de la desigualdad del desempeño educativo (20002012).

\begin{tabular}{|c|c|c|c|c|c|}
\hline \multirow{2}{*}{$\begin{array}{l}\text { Descomposición } \\
\text { Medida de desigualdad }\end{array}$} & \multicolumn{5}{|c|}{ 2012-2000 } \\
\hline & $90-10$ & $50-10$ & $90-50$ & Varianza & Gini \\
\hline Cambio sin ajustar & $\begin{array}{l}-94.46 \\
(0.72)^{* * *}\end{array}$ & $\begin{array}{l}-58.88 \\
(0.62)^{* * *}\end{array}$ & $\begin{array}{l}-35.58 \\
(0.44)^{* * *}\end{array}$ & $\begin{array}{l}-7669.90 \\
(42.42)^{* * *}\end{array}$ & $\begin{array}{l}-0.0593 \\
(0.0003)^{* * *}\end{array}$ \\
\hline \multicolumn{6}{|l|}{ Efecto composición atribuido a } \\
\hline Género & $\begin{array}{l}2.26 \\
(0.72)^{* * *}\end{array}$ & $\begin{array}{l}-0.48 \\
(0.24)^{* *}\end{array}$ & $\begin{array}{l}2.74 \\
(0.19) * * *\end{array}$ & $\begin{array}{l}80.21 \\
(15.82)^{* * *}\end{array}$ & $\begin{array}{l}0.0004 \\
(0.0001)^{* * *}\end{array}$ \\
\hline Ocupación y educación de padres & $\begin{array}{l}-8.73 \\
(0.72)^{* * *}\end{array}$ & $\begin{array}{l}-4.57 \\
(0.73)^{* * *}\end{array}$ & $\begin{array}{l}-4.16 \\
(0.70)^{* * *}\end{array}$ & $\begin{array}{l}-553.88 \\
(43.69)^{* * *}\end{array}$ & $\begin{array}{l}-0.0057 \\
(0.0004)^{* * *}\end{array}$ \\
\hline Status migratorio e idioma & $\begin{array}{l}0.01 \\
(0.72)\end{array}$ & $\begin{array}{l}0.21 \\
(0.11)^{* *}\end{array}$ & $\begin{array}{l}-0.20 \\
(0.08) * *\end{array}$ & $\begin{array}{l}32.16 \\
(7.92)^{* * *}\end{array}$ & $\begin{array}{l}0.0000 \\
-0.0001\end{array}$ \\
\hline Localización & $\begin{array}{l}0.16 \\
(0.72)\end{array}$ & $\begin{array}{l}0.64 \\
(0.29)^{* *}\end{array}$ & $\begin{array}{l}-0.48 \\
(0.21)^{* *}\end{array}$ & $\begin{array}{l}-23.32 \\
(14.79)\end{array}$ & $\begin{array}{l}0.0000 \\
(0.0001)\end{array}$ \\
\hline Recursos & $\begin{array}{l}19.96 \\
(0.72)^{* * *}\end{array}$ & $\begin{array}{l}19.84 \\
(1.01)^{* * *}\end{array}$ & $\begin{array}{l}0.12 \\
(0.82)\end{array}$ & $\begin{array}{l}1590.66 \\
(64.72)^{* * *}\end{array}$ & $\begin{array}{l}0.0037 \\
(0.0004)^{* * *}\end{array}$ \\
\hline \multicolumn{6}{|l|}{ Aceptables: } \\
\hline Edad & $\begin{array}{l}1.93 \\
(0.27)^{* * *}\end{array}$ & $\begin{array}{l}2.44 \\
(0.23)^{* * *}\end{array}$ & $\begin{array}{l}-0.51 \\
(0.19)^{* * *}\end{array}$ & $\begin{array}{l}156.20 \\
(16.63)^{* * *}\end{array}$ & $\begin{array}{l}-0.0002 \\
(0.0001)^{*}\end{array}$ \\
\hline Año que cursa & $\begin{array}{l}17.40 \\
(1.04)^{* * *}\end{array}$ & $\begin{array}{l}10.38 \\
(0.86)^{* * *}\end{array}$ & $\begin{array}{l}7.02 \\
(0.39)^{* * *}\end{array}$ & $\begin{array}{l}1388.98 \\
(65.06)^{* * *}\end{array}$ & $\begin{array}{l}0.0150 \\
(0.0006)^{* * *}\end{array}$ \\
\hline Total efecto composición & $\begin{array}{l}\mathbf{3 2 . 9 9} \\
(1.74)^{* * *}\end{array}$ & $\begin{array}{l}\mathbf{2 8 . 4 6} \\
(1.38)^{* * *}\end{array}$ & $\begin{array}{l}4.53 \\
(1.14)^{* * *}\end{array}$ & $\begin{array}{l}\mathbf{2 6 7 1 . 0 2} \\
(93.06)^{* * *}\end{array}$ & $\begin{array}{l}\mathbf{0 . 0 1 3 2} \\
(0.0008)^{* * *}\end{array}$ \\
\hline \multicolumn{6}{|l|}{ Efecto parámetro atribuido a } \\
\hline Género & $\begin{array}{l}-14.86 \\
(0.72)^{* * *}\end{array}$ & $\begin{array}{l}5.66 \\
(2.20)^{* * *}\end{array}$ & $\begin{array}{l}-20.52 \\
(1.15)^{* * *}\end{array}$ & $\begin{array}{l}14.65 \\
(84.23)\end{array}$ & $\begin{array}{l}-0.0043 \\
(0.0006)^{* * *}\end{array}$ \\
\hline Ocupación y educación de los padres & $\begin{array}{l}-41.02 \\
(0.72)^{* * *}\end{array}$ & $\begin{array}{l}12.70 \\
(19.46)\end{array}$ & $\begin{array}{l}-53.72 \\
(4.89)^{* * *}\end{array}$ & $\begin{array}{l}-8610.66 \\
(447.69)^{* * *}\end{array}$ & $\begin{array}{l}-0.0936 \\
(0.0034) * * *\end{array}$ \\
\hline Status migratorio e idioma & $\begin{array}{l}-3.64 \\
(0.72)^{* * *}\end{array}$ & $\begin{array}{l}0.85 \\
(0.73)\end{array}$ & $\begin{array}{l}-4.49 \\
(0.73)^{* * *}\end{array}$ & $\begin{array}{l}-30.30 \\
(41.79)\end{array}$ & $\begin{array}{l}0.0016 \\
(0.0003)^{* * *}\end{array}$ \\
\hline Localización & $\begin{array}{l}12.79 \\
(0.72)^{* * *}\end{array}$ & $\begin{array}{l}-18.44 \\
(2.58)^{* * *}\end{array}$ & $\begin{array}{l}31.23 \\
(2.47)^{* * *}\end{array}$ & $\begin{array}{l}2358.04 \\
(103.28)^{* * *}\end{array}$ & $\begin{array}{l}0.0134 \\
(0.0008)^{* * *}\end{array}$ \\
\hline Recursos & $\begin{array}{l}-25.61 \\
(0.72)^{* * *}\end{array}$ & $\begin{array}{l}-45.43 \\
(8.29)^{* * *}\end{array}$ & $\begin{array}{l}19.82 \\
(2.23) * * *\end{array}$ & $\begin{array}{l}4599.91 \\
(263.54)^{* * *}\end{array}$ & $\begin{array}{l}0.0477 \\
(0.0017)^{* * *}\end{array}$ \\
\hline \multicolumn{6}{|l|}{ Aceptables: } \\
\hline Edad & $\begin{array}{l}1724.33 \\
(176.08)^{* * *}\end{array}$ & $\begin{array}{l}714.17 \\
(189.46)^{* * *}\end{array}$ & $\begin{array}{l}1010.16 \\
(79.37)^{* * *}\end{array}$ & $\begin{array}{l}77308.03 \\
(4071.99)^{* * *}\end{array}$ & $\begin{array}{l}1.0428 \\
(0.0322)^{* * *}\end{array}$ \\
\hline Año que cursa & $\begin{array}{l}135.32 \\
(13.71)^{* * *}\end{array}$ & $\begin{array}{l}138.00 \\
(13.96)^{* * *}\end{array}$ & $\begin{array}{l}-2.68 \\
(4.52)\end{array}$ & $\begin{array}{l}5003.76 \\
(121.33)^{* * *}\end{array}$ & $\begin{array}{l}0.0477 \\
(0.0010)^{* * *}\end{array}$ \\
\hline Constante & $\begin{array}{l}-1927.55 \\
(0.72)^{* * *}\end{array}$ & $\begin{array}{l}-926.55 \\
(181.84)^{* * *}\end{array}$ & $\begin{array}{l}-1001.00 \\
(76.59)^{* * *}\end{array}$ & $\begin{array}{l}-88059.92 \\
(3878.43)^{* * *}\end{array}$ & $\begin{array}{l}-1.1092 \\
(0.0304)^{* * *}\end{array}$ \\
\hline Total efecto parámetros & $\begin{array}{l}\mathbf{- 1 4 0 . 2 3} \\
(4.23)^{* * *} \\
\end{array}$ & $\begin{array}{l}\mathbf{- 1 1 9 . 0 3} \\
(3.60)^{* * *} \\
\end{array}$ & $\begin{array}{l}\mathbf{- 2 1 . 2 0} \\
(2.23)^{* * *}\end{array}$ & $\begin{array}{l}\mathbf{- 7 4 1 6 . 4 8} \\
(188.35)^{* * *}\end{array}$ & $\begin{array}{l}-\mathbf{0 . 0 5 3 8} \\
(0.0017)^{* * *}\end{array}$ \\
\hline Error de reponderación & $\begin{array}{l}-7.87 \\
(0.72)^{* * *}\end{array}$ & $\begin{array}{l}-0.55 \\
(0.50)\end{array}$ & $\begin{array}{l}-7.32 \\
(1.00) * * *\end{array}$ & $\begin{array}{l}-1085.77 \\
(83.36)^{* * *}\end{array}$ & $\begin{array}{l}-0.0097 \\
(0.0007)^{* * *}\end{array}$ \\
\hline Error de especificación & $\begin{array}{l}20.65 \\
(3.91)^{* * *}\end{array}$ & $\begin{array}{l}32.24 \\
(3.79)^{* * *}\end{array}$ & $\begin{array}{l}-11.59 \\
(2.46)^{*}\end{array}$ & $\begin{array}{l}-1838.67 \\
(126.24)^{* * *}\end{array}$ & $\begin{array}{l}-0.0090 \\
(0.0009) * * *\end{array}$ \\
\hline
\end{tabular}

Nota: Resultados en base a estimaciones del modelo 1. Dicho modelo incluye como variables socialmente no aceptables al género, educación y ocupación de los padres junto con estructura del hogar, estatus migratorio, recursos educativos, localización geográfica y como variables socialmente aceptables la edad y año escolar. Errores estándar con bootstrap (200 repeticiones) entre paréntesis. $*$ significativo al $10 \%$; ** significativo al $5 \%$; *** significativo al $1 \%$.

Fuente: Elaboración propia en base a datos PISA.

El Gráfico 2.4 muestra la contribución de cada una de las variables no aceptables al cambio del coeficiente de Gini entre 2000 y 2012. Se observa que la localización de la escuela junto con los recursos educativos son las principales variables que contribuyeron al aumento de la desigualdad en dicho periodo.

La descomposición permite indagar cómo ambas variables han incidido en la desigualdad del desempeño educativo. La localización de la escuela contribuyó al aumento del coeficiente de Gini, principalmente, a través del efecto parámetros. El efecto composición de la localización es casi nulo, mostrando que la proporción de alumnos que asisten a escuelas en pueblos 
pequeños/medianos y ciudades no dan cuenta de los cambios de desigualdad sino que dichos cambios están relacionados con los retornos de la localización geográfica de dichas escuelas. Con lo cual pareciera que características más estructurales inherentes a las zonas geográficas son las que generan cambios en el desempeño educativo más que variaciones en la distribución de alumnos entre los distintos pueblos y ciudades.

A partir de las regresiones de cuantiles de RIF, se advierte que el coeficiente estimado asociado a las variables de localización es negativo, evidenciando para los distintos cuantiles que asistir a escuelas localizadas en pueblos pequeños o medianos tiene un efecto negativo en el desempeño educativo respecto a alumnos que asisten a escuelas localizadas en ciudades. Si se tienen en cuenta los resultados de las regresiones de RIF del año 2000 y se comparan con las estimaciones del año 2012, se encuentra que en 2012 el efecto negativo es menor en valor absoluto en los cuantiles extremos superiores de la distribución respecto al año 2000. Este menor efecto amplía la brecha entre los cuantiles superiores y el resto de los cuantiles dando como resultado en las descomposiciones de cuantiles un efecto parámetros también positivo (ver Tabla 2.4).

Otros factores que también contribuyeron al aumento de la desigualdad de oportunidades son los recursos educativos del hogar. El análisis de la descomposición del coeficiente de Gini sugiere que tanto las dotaciones como los retornos (estos últimos en mayor medida), contribuyeron a aumentar la desigualdad. Si se analiza la brecha de cuantiles 90-10, el efecto composición arroja un resultado positivo mostrando que las dotaciones ampliaron esta brecha. Por un lado, poseer una computadora o Internet en el hogar en los últimos años es algo más común que en el año 2000. En el año 2000, el $48 \%$ de los alumnos de la muestra tenían en su hogar una computadora y el $24 \%$ Internet mientras que en 2012 estos porcentajes ascienden a $85 \%$ y $77 \%$, respectivamente. ${ }^{8}$ Con lo cual es esperable que las diferencias de dotación entre los cuantiles superiores y medios sean más pequeñas en 2012 que en el año 2000 y que el efecto de esta variable sea más alto en los cuantiles del extremo inferior de la distribución y, por ello, es que se observa un efecto composición alto en la brecha de cuantiles 50-10. Este efecto en el lado izquierdo de la distribución lleva a que el efecto composición sea mayor en el caso de la brecha 90-10 que en el caso del coeficiente de Gini. Por otro lado, los resultados sugieren que los retornos de los recursos educativos han contribuido a disminuir la brecha entre los cuantiles más bajos y los cuantiles centrales de la distribución pero que en el global han aumentado la desigualdad. En cuanto a la variable libros, la misma ha contribuido al aumento de la desigualdad en el periodo. El retorno de libros en el hogar, positivo monótono creciente entre los cuantiles, se ha acentuado en el periodo contribuyendo también a un aumento del coeficiente de Gini.

Las desigualdades socioeconómicas de los padres de los alumnos argentinos permiten explicar en su conjunto una proporción apreciable de la disminución de la desigualdad de los puntajes. Por otro lado, pareciera que el estatus migratorio e idioma no juegan un rol relevante en la desigualdad de oportunidades ya que las contribuciones de estas variables son relativamente pequeñas.

${ }^{8}$ Estos porcentajes, pueden en parte, estar asociados al Programa Conectar Igualdad que se creó en 2010 que proporciona una computadora a cada alumno y docente de escuelas públicas secundarias de todo el país. Sin embargo, ya en el 2009 este porcentaje ascendía a $70 \%$ y $53 \%$ de los alumnos de la muestra de PISA. 
Gráfico 2.4: Contribución de las variables no aceptables al coeficiente de Gini según estimaciones del modelo 1 .

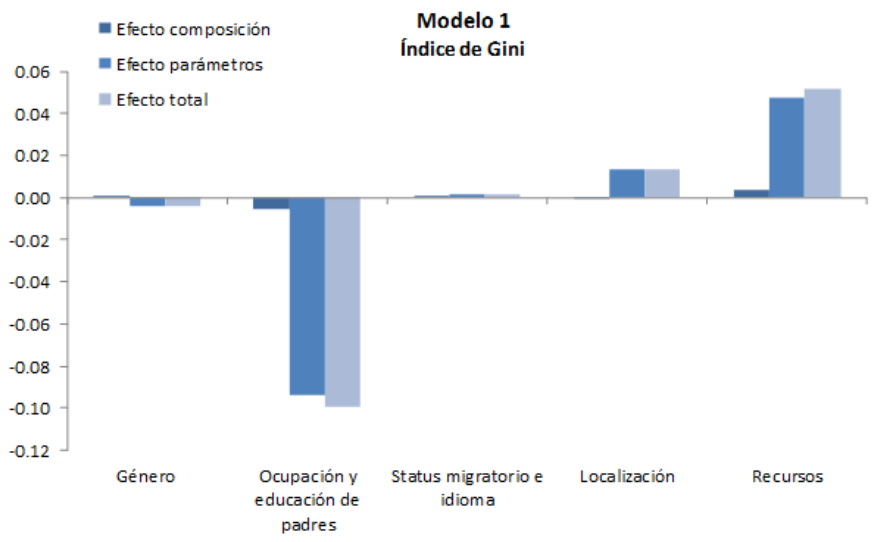

Nota: El modelo 1 incluye como variables explicativas socialmente no aceptables al género, educación y ocupación de los padres junto con estructura del hogar, estatus migratorio, recursos educativos y localización geográfica e incluye como aceptables las variables edad del estudiante y añotura del

Fuente: Elaboración propia en base a datos PISA.

Las descomposiciones en base a las estimaciones de los modelos 2 y 3 que incorporan como variable no aceptable el contexto socioeconómico del colegio muestran que esta variable ha contribuido a aumentar la desigualdad de oportunidades aunque levemente. Como era de esperar en las descomposiciones de ambos modelos no se observan cambios en el efecto composición respecto al modelo 1, pero sí se observan cambios en la composición del efecto parámetros. En particular, el efecto de las características de los padres (educación y ocupación de los padres y estructura del hogar) así como de la localización es menor al encontrado en el modelo 1. A pesar de estos cambios, la descomposición de la desigualdad global del coeficiente de Gini no se ve modificada significativamente. En el Gráfico 2.5 se puede observar que tanto en el modelo 2 como en el modelo 3 la localización y los recursos siguen siendo los principales factores asociados al incremento del coeficiente de Gini a través del efecto parámetros.

Gráfico 2.5: Contribución de las variables no aceptables al coeficiente de Gini según estimaciones de los modelos 2 y 3.

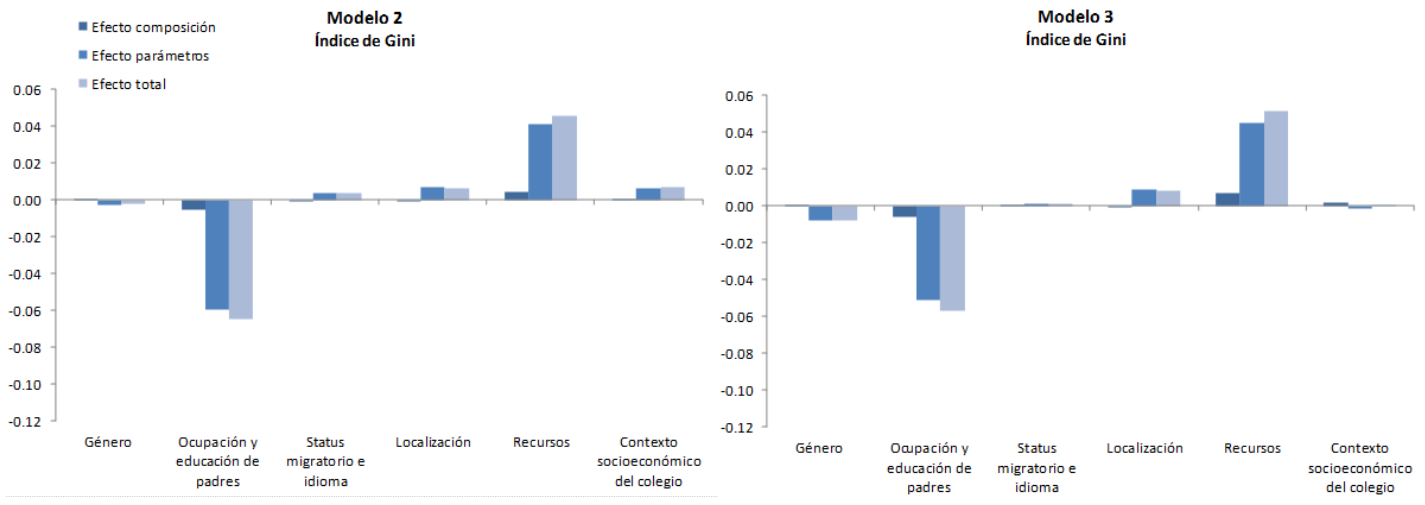

Nota: El modelo 2 incluye como variables explicativas socialmente no aceptables al género, educación y ocupación de los padres junto con estructura del hogar, estatus migratorio, recursos educativos, localización geográfica y contexto socioeconómico del colegio e incluye como aceptables las variables edad del estudiante y año escolar. El modelo 3 incluye todas las variables del modelo 2 e incorpora la variable aceptable tiempo de estudio del alumno.

Fuente: Elaboración propia en base a datos PISA.

Al sumar todos los efectos de las variables no aceptables y aceptables, es posible analizar 
la dirección global de los mismos e identificar situaciones de aumento de desigualdad de oportunidades (la desigualdad de oportunidades habrá aumentado si la contribución total de las variables no aceptables es positiva independientemente del cambio total de la distribución). En el Gráfico 2.6 se presentan las contribuciones totales de cada grupo de variables sobre las brechas de cuantiles, la varianza y el coeficiente de Gini. Esto se realiza para cada modelo estimado.

El modelo 1 no presenta evidencia de un aumento de la desigualdad de oportunidades. Si bien la localización y los recursos contribuyeron a aumentar la desigualdad entre 2000 y 2012, estos efectos se contrarrestaron con los de las demás variables consideradas. A partir del modelo 2 se encuentra que las variables socialmente no aceptables contribuyeron a ampliar la brecha entre los cuantiles 90-10, debido principalmente al efecto parámetro de la educación y ocupación de los padres. El mayor impacto se da en el lado izquierdo de la distribución (brecha 50-10). Por su parte, el modelo 3 sugiere que la desigualdad de oportunidades ha aumentado particularmente a través de las brechas entre cuantiles a partir del efecto parámetro de la educación y ocupación de los padres y los recursos educativos.

En cuanto a la desigualdad global medida a partir de la varianza y el coeficiente de Gini, se encuentra que las variables no aceptables, en la mayoría de los casos, han contribuido en forma conjunta a disminuir la desigualdad. Sin embargo, esta contribución ha sido pequeña en relación a la contribución de las variables aceptables. En todos los casos, se encuentra que la contribución total de las variables aceptables es mayor a la de las variables no aceptables. Sugiriendo que la caída de la desigualdad total del desempeño educativo se debe principalmente a la contribución de las variables aceptables y el término residual. ${ }^{9}$

Gráfico 2.6: Contribución total de factores no aceptables y factores aceptables.

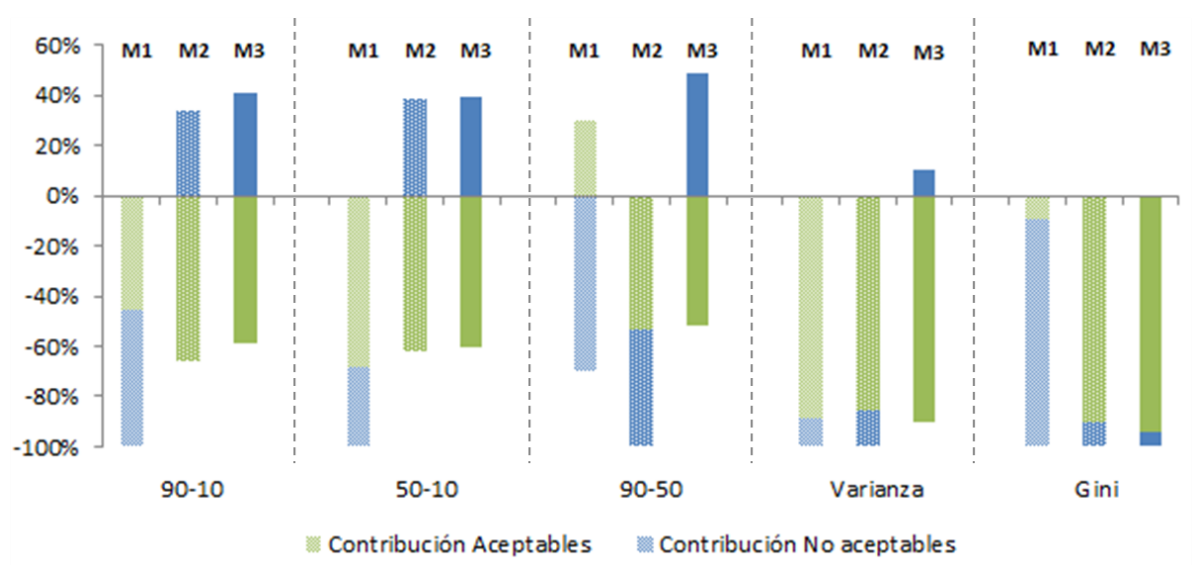

Nota: El modelo 1 incluye como variables explicativas socialmente no aceptables al género, educación y ocupación de los padres junto con estructura del hogar, estatus migratorio, recursos educativos y localización geográfica e incluye como aceptables las variables edad del estudiante y año escolar. El modelo 2 incluye todas las variables del modelo 1 y agrega como variable no aceptable el contexto socioeconómico del colegio. El modelo 3 incluye todas las variables del modelo 2 e incorpora la variable aceptable tiempo de estudio del alumno. Fuente: Elaboración propia en base a datos PISA.

Como ejercicio complementario, es posible extender este análisis a otros dos sub-periodos de tiempo: 2006-2012 y 2009-2012. Estos dos sub-periodos permiten analizar qué ha sucedido con la desigualdad de oportunidades luego de la crisis argentina del año 2001. La siguiente Tabla 2.5 presenta los principales resultados de las descomposiciones encontrados para estos dos períodos.

\footnotetext{
${ }^{9}$ Resultados similares se encuentran para lectura y ciencia. En particular, en estas dos competencias se observa que el efecto de los padres es mayor al encontrado en matemáticas y nuevamente son las variables aceptables las que más han contribuido a disminuir la desigualdad.
} 
Tabla 2.5: Resumen de la descomposición de la desigualdad del desempeño educativo.

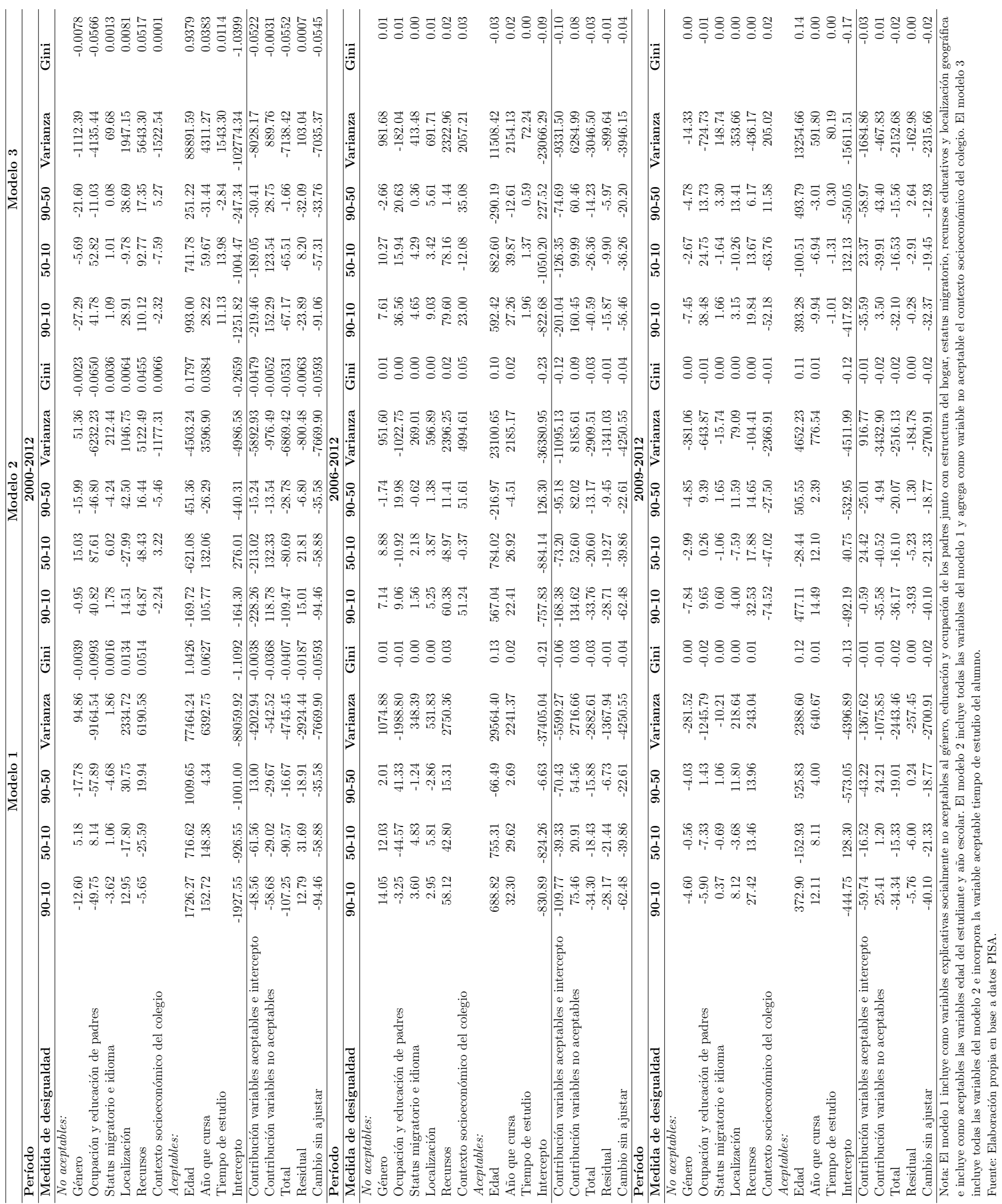

En síntesis, se encuentra que las variables no aceptables aumentaron la desigualdad entre 2006-2012 y 2009-2012. Esto sugiere que a pesar de la caída en la desigualdad total de los puntajes de matemáticas, la desigualdad de oportunidades en estos periodos aumentó. Entre 2006 y 2012 las variables no aceptables contribuyeron a ampliar las brechas de cuantiles, la 
varianza y el coeficiente de Gini. Asimismo, entre 2009 y 2012, dichas variables contribuyeron a aumentar el coeficiente de Gini y las brechas 90-10 y 90-50, teniendo mayor impacto en el lado derecho de la distribución.

Si bien el efecto de las variables socialmente no aceptables no es homogéneo y depende de la parte de la distribución y medida estadística que se esté analizando, del análisis se puede concluir que la localización de la escuela y los recursos educativos son los factores que explican los cambios en la desigualdad de oportunidades entre los años 2000-2012 y en los subperiodos 2006-2012 y 2009-2012. También se encuentra que el contexto socioeconómico del alumno tuvo un impacto negativo favoreciendo el aumento de la desigualdad principalmente medida por la varianza y coeficiente de Gini en los sub-periodos 2006-2012 y 2009-2012.

\subsubsection{Extensiones}

En este apartado se compara Argentina con otros países de América Latina y se analiza si ha ocurrido algo similar en dichos países durante los 2000s. Los países que se consideraron para el estudio son Brasil, Chile y México, ya que participaron en la primer edición de PISA y se cuenta con datos del año 2000.

Al igual que en Argentina, la desigualdad de los puntajes de matemáticas en estos países se ha reducido. Como se observa en el Gráfico 2.7, entre 2000 y 2012, el coeficiente de Gini de cada país ha disminuido. Algo similar también se encuentra en otros índices como la varianza o brechas de cuantiles (ver Tabla A2.5 del Apéndice 2).

\section{Gráfico 2.7: Evolución del coeficiente de Gini de los puntajes de matemáticas según países.}

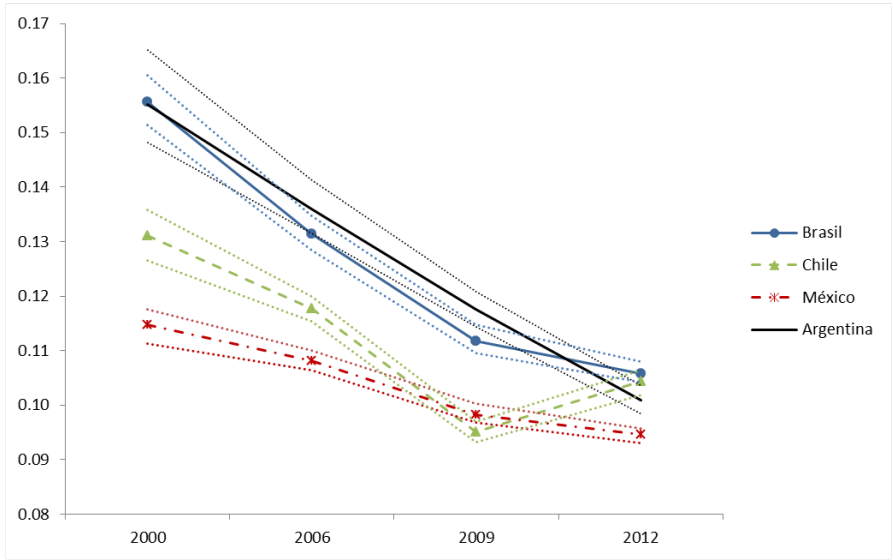

Nota: Líneas punteadas corresponden a intervalos de confianza del $95 \%$ con bootstrap (200 repeticiones). Fuente: Elaboración propia en base a datos PISA.

Con el fin de analizar las fuentes de este cambio distributivo se lleva a cabo la descomposición para cada país en base al modelo más completo (modelo 3). La Tabla 2.6 presenta los principales resultados de la descomposición del cambio de las brechas entre cuantiles, la varianza y el coeficiente de Gini entre los años 2000 y $2012 .^{10}$

\footnotetext{
${ }^{10}$ La tabla completa se encuentra en el Apéndice 2.
} 
Tabla 2.6: Descomposición de la desigualdad del desempeño educativo por país (2000-2012).

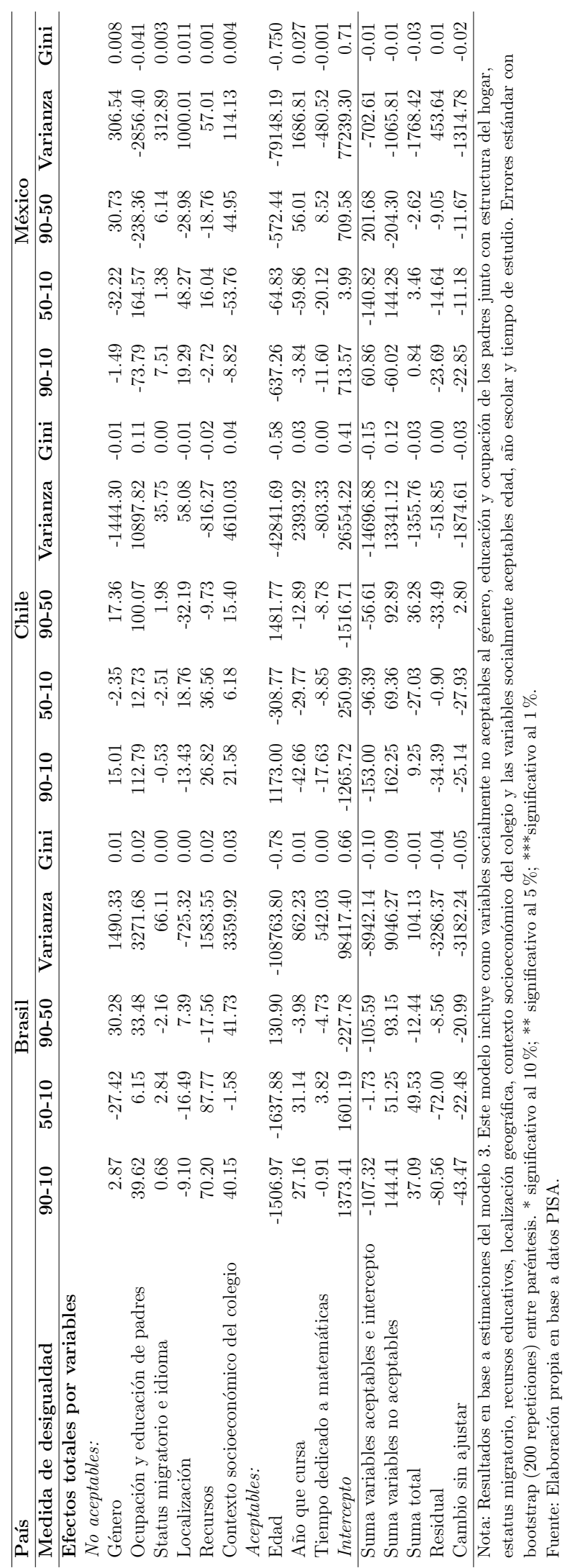

A diferencia de Argentina, se encuentra que el contexto socioeconómico del colegio es un factor importante que contribuye al aumento de desigualdad en estos países. Tanto en Brasil 
como en Chile se encuentra que las variables no aceptables han contribuido a aumentar la desigualdad en el periodo considerado. Las variables que muestran una mayor contribución a la desigualdad global medida por el índice de Gini y la varianza son las características de los padres y el contexto socioeconómico del colegio.

En el caso de México se observa que el género, la localización de la escuela, el estatus migratorio y los recursos educativos han contribuido al aumento de la desigualdad mientras que las características de los padres (educación y ocupación de los padres y estructura del hogar) han contribuido a la disminución de la misma. En el global estos efectos se han compensado dando como resultado una disminución de la desigualdad de oportunidades debido principalmente a los efectos de las características de los padres. En este caso la desigualdad de oportunidades ha disminuido contribuyendo a la caída global de la desigualdad educativa mexicana.

\subsection{Conclusiones}

A la hora de examinar el grado de desigualdad de oportunidades de una sociedad, resulta útil conocer cómo distintas variables contribuyen a la desigualdad de resultados. Los diferentes factores subyacentes de cada distribución pueden impactar distributivamente de forma muy distinta, algunos de ellos pueden generar una desigualdad socialmente aceptable y otros una desigualdad no aceptable. Las microdescomposiciones permiten identificar los efectos individuales de cada uno de estos factores en la distribución.

Este capítulo analiza la desigualdad del desempeño educativo en Argentina a partir de microdescomposiciones utilizando regresiones de funciones de influencia recentradas (RIF). El método de descomposición empleado es lo bastante flexible para analizar cambios distributivos a partir de diferentes estadísticos. Se utilizan microdatos a nivel alumno provenientes de la base de datos del programa de evaluación PISA de la OCDE. Como medida del desempeño educativo se consideran los puntajes en matemáticas de las pruebas PISA y se proponen tres modelos de desempeño. El primer modelo incluye las variables explicativas: género, conformación del hogar, educación de los padres, ocupación de los padres, estatus migratorio, localización del colegio, recursos educativos, edad y año escolar. El segundo modelo incluye también el contexto socioeconómico del colegio y el tercer modelo incorpora el contexto del colegio y el esfuerzo del alumno aproximado por el tiempo que dedica al estudio según su propio reporte.

Si se analizan los datos se encuentra que la desigualdad de los puntajes de matemática de los alumnos de Argentina ha disminuido entre los años 2000 y 2012. La descomposición sugiere que las variables aceptables consideradas en este trabajo son las que han contribuido en mayor medida a reducir dicha desigualdad mientras que las variables no aceptables han contribuido en menor medida. Esto indicaría que en los últimos años Argentina no ha podido reducir significativamente la desigualdad de oportunidades educativas asociada a los factores no aceptables definidos en este estudio. Más aún, se advierte en los tres modelos propuestos que los recursos educativos y la localización han contribuido en forma individual a aumentar la desigualdad de oportunidades en estos años. A diferencia de lo que se esperaba los resultados sugieren que las características de los padres no han contribuido al aumento de la desigualdad global medida por el coeficiente de Gini. Estas variables solo han contribuido a profundizar las brechas entre los cuantiles extremos. Si se extiende el análisis a los sub-periodos 2006-2012 y 2009-2012, se obtiene que la desigualdad de oportunidades ha aumentado particularmente en el sub-periodo 2006-2012.

El análisis también se extendió a otros países de América Latina. Los resultados indican que, a diferencia de Argentina, en Chile y Brasil las variables no aceptables contribuyeron al 
aumento de la desigualdad de oportunidades entre los años 2000 y 2012. En ambos países la educación y la ocupación de los padres fueron factores relevantes en este incremento. En el caso de México, se encuentra que la educación y ocupación de los padres y la estructura del hogar contribuyeron a disminuir la desigualdad de oportunidades compensando los efectos de las demás variables no aceptables.

A partir de los resultados de las descomposiciones, es posible identificar factores que pueden ser manejables (o no) por la política educativa para reducir la desigualdad. Los recursos educativos y tecnológicos como libros, computadoras e Internet podrían ser factores controlables por la política. Es así que se puede pensar en intervenciones que compensen desventajas del hogar con recursos provistos por el Estado como, por ejemplo, el Programa Conectar Igualdad creado en el año 2010 que entregó computadoras.

A partir de las estimaciones se observa que las dotaciones de los recursos educativos incrementan, principalmente, la brecha de cuantiles 50-10; con lo cual los programas destinados a proporcionar recursos educativos a los alumnos, no solo tienen que tener como objetivo incrementar las tasas de utilización de computadoras, Internet o libros, sino también tienen que focalizarse en los alumnos con menores posibilidades de éxito en las pruebas. El impacto potencial de corto plazo de este tipo de política aplicaría especialmente a disminuir las brechas en la parte izquierda de la distribución de desempeño educativo. Según las estimaciones del trabajo una mayor utilización de recursos educativos podría asociarse con una leve disminución de la desigualdad global (menos que 0.01 punto del coeficiente de Gini). Es por eso, que la misma debería ser considerada como solo una medida complementaria de corto plazo para disminuir la desigualdad de oportunidades de los jóvenes con menos ventajas.

En cuanto a los efectos estructurales de este tipo de intervenciones, existe evidencia mixta sobre el efecto causal de los recursos educativos en el desempeño (Angrist y Lavy, 2002; Banerjee et al., 2005; Fuchs y Wößmann, 2004, 2007; Leuven et al., 2007; Glewwe et al., 2009; Vigdor et al., 2014). Además, los resultados de las descomposiciones sugieren que los recursos educativos tienen un impacto diferente según la parte de la distribución que se analice, mostrando que los recursos tienen un menor efecto positivo en los alumnos que presentan puntajes más bajos en las pruebas. Con lo cual estos programas deberían asegurar el correcto uso de los recursos educativos brindados, con el fin de asegurar a todos los alumnos el máximo retorno de dichos recursos ya que, generalmente, se encuentra que la manera en que estos recursos se incorporan al proceso educativo es lo que hace que sean o no efectivos. Este tipo de intervención sería más de largo plazo que de corto plazo y su diseño e implementación requiere de una mayor complejidad.

En cuanto a la localización del colegio éste no es un factor fácilmente manipulable por la política educativa. Más aún, el mayor incremento de desigualdad en la última década ha provenido de diferencias estructurales (efecto parámetros). Por lo tanto, en este caso son necesarias medidas compensatorias de largo plazo que tiendan a reducir desequilibrios regionales asociados a diferencias entre zonas urbanas y pueblos pequeños/medianos. Seguramente esta variable pueda estar capturando también otros efectos asociados a la localización del colegio como la calidad docente, organización y accesibilidad del colegio, entre otros, que sí son manejables. Por lo tanto, habría que analizar las ventajas que tienen los establecimientos educativos por encontrarse en zonas urbanas que no tienen los colegios que se ubican en pueblos medianos y pequeños y viceversa, con el fin de diseñar políticas compensatorias que equilibren los efectos desigualadores de la localización geográfica. A su vez en Argentina existen heterogeneidades locales, hay escuelas en jurisdicciones pobres y ricas, y esto puede también estar afectando de alguna manera los resultados, y debería ser tenido en cuenta en la política compensatoria. 


\section{CAPÍTULO 3}

\section{El papel de las escuelas públicas y privadas en la igualdad de oportunidades en Argentina. Un análisis de dominancia estocástica.}

\subsection{Introducción}

Argentina ha experimentado importantes cambios en su sistema educativo durante los últimos años. La participación del sector privado en la matrícula total de alumnos ha aumentado considerablemente. Según datos de los anuarios estadísticos de la DINIECE (2015) la cantidad de alumnos en establecimientos públicos aumentó, entre 2000 y 2014, un $11 \%$ mientras que la cantidad de alumnos en colegios privados aumentó un $20 \%$. A su vez ha habido un cambio en la composición de los alumnos en las escuelas públicas. Los alumnos que pertenecen a estratos socioeconómico medios y altos se han volcado a la educación privada, generando así una mayor segregación escolar por nivel socioeconómico entre escuelas públicas y privadas (Gasparini et al., 2011).

Asimismo, en general, el desempeño educativo de los alumnos que asisten a escuelas privadas, es en promedio más alto respecto a aquellos que asisten a escuelas públicas. El Programa Internacional para la Evaluación de Estudiantes (PISA, por sus siglas en inglés) realizado en el año 2012 ofrece un primer panorama sobre estas diferencias. Los datos relevados muestran que los puntajes promedios de matemática, lectura y ciencia de los alumnos que asisten a establecimientos educativos privados son alrededor de 20 puntos porcentuales más altos que los puntajes de los alumnos de las escuelas públicas.

Estas diferencias entre escuelas públicas y privadas pueden ser una fuente de desigualdad de oportunidades si: a) el acceso a la educación privada está asociado a la condición socioeconómica, y b) la "calidad educativa", controlando por características individuales, es mayor en la escuela privada. Este estudio tiene como objetivo analizar si en la actualidad la escuela pública sigue teniendo el rol igualador de oportunidades que la sociedad le ha asociado históricamente; examinar si las diferencias en el desempeño educativo entre escuelas públicas y privadas se mantienen en el caso de alumnos que se esfuerzan igual; y verificar si estas diferencias continúan incluso controlando por otras variables asociadas a características familiares y contexto educativo del alumno.

Se comparan distribuciones de resultados educativos de distintos grupos de alumnos condicionando por un conjunto de variables de circunstancias y esfuerzo. Como resultados educativos se toman los puntajes de las pruebas PISA de matemática, lectura y ciencia. 
Las variables de circunstancias, asociadas a factores no aceptables, que se consideran son: el tipo de establecimiento (público, privado subvencionado, privado independiente), el nivel educativo máximo alcanzado por los padres del alumno, el nivel socioeconómico cultural familiar y el clima educativo del colegio. A partir de estas variables y sus respectivas combinaciones se generan diferentes grupos de alumnos que se caracterizan por tener las mismas circunstancias.

Siguiendo a Roemer (1998), se entiende por igualdad de oportunidades (IOP) a una situación donde dos grupos de alumnos que solo difieren en circunstancias tienen las mismas probabilidades de alcanzar determinados resultados educativos. Esta definición implica seguir un criterio fuerte ya que, principalmente, se plantea que las funciones de distribución acumulada del desempeño educativo de ambos grupos sean iguales. En la realidad son pocos los casos donde dichas funciones resultan iguales. Por ello es que también se trabaja con otras definiciones más débiles de IOP. Algunas de estas definiciones más débiles postulan que existirá IOP si no es posible ordenar y preferir una distribución de resultados educativos de un grupo sobre otra distribución de otro grupo utilizando criterios de dominancia estocástica.

Lefranc et al. (2009), retoman la idea de Checchi y Peragine (2005) y consideran grupos de individuos que comparten las mismas circunstancias y utilizan distribuciones condicionadas de ingresos. Los autores proveen condiciones necesarias y suficientes para la existencia de IOP ya sea en sentido fuerte o débil y comparan las distribuciones de los diferentes grupos siguiendo principios de dominancia estocástica de primer orden y segundo orden. También incorporan al análisis la suerte de los individuos como una forma de riesgo. Para la aplicación empírica utilizan datos de Francia de los años 1979-2000. A pesar de que encuentran que la herencia social es una relevante fuente de desigualdad, no encuentran evidencia significativa de IOP en ingresos a través de los tests de dominancia.

En cuanto al esfuerzo, si bien la teoría de Roemer establece que es un factor relevante en la distribución de resultados, gran parte de la literatura empírica se ha concentrado en analizar la IOP a partir de las circunstancias de los individuos. Ravallion (2015) realiza un análisis del efecto del esfuerzo en la teoría de distribución y explora las implicaciones de medir la desigualdad (ya sea de resultados o de oportunidades) explotando la heterogeneidad de los esfuerzos individuales. Encuentra que ajustando por el esfuerzo, la desigualdad es menor aunque el efecto de dicho factor es muy pequeño y señala que en un principio la dirección del sesgo de este factor puede no ser obvia.

Este capítulo analiza la igualdad de oportunidades entre alumnos de escuelas públicas y privadas teniendo en cuenta las circunstancias así como también el esfuerzo individual de los mismos. Incorporar medidas de esfuerzo empíricamente no resulta una tarea sencilla. Principalmente, esto se debe a que en general las bases de datos no proveen información adecuada sobre los esfuerzos individuales. A pesar de ello, en el caso de la educación es posible trabajar con alguna aproximación del esfuerzo individual del alumno. Por ejemplo, se puede tomar la cantidad de horas que el alumno dedica a estudiar una materia ya que esto puede implicar un mayor o menor esfuerzo individual.

Las siguientes secciones se organizan de la siguiente manera: en la sección 2 se presenta la metodología a utilizar, en la sección 3 se describen los datos y en la sección 4 se exhiben los principales resultados. En la sección 5 se presentan las principales conclusiones del trabajo.

\subsection{Metodología}

La teoría de IOP se basa en analizar cuáles son los factores que generan la distribución de resultados de los individuos, como ya se ha mencionado estos factores se pueden dividir 
en esfuerzo y circunstancias (o factores socialmente aceptables y no aceptables). Si las distribuciones de resultados son independientes de las circunstancias y la desigualdad observada es solo resultado de los distintos esfuerzos de los individuos y la "suerte", entonces se podría concluir que existe algún tipo de IOP (débil o fuerte). ${ }^{1}$

En esta sección se presenta una definición fuerte de IOP y definiciones más débiles que se tendrán en cuenta en este capítulo. Luego, se exponen los criterios de dominancia estocástica para analizar la existencia de IOP, el método de estimación, las pruebas de hipótesis a testear y la forma en que se procederá a comparar las distintas distribuciones de resultados educativos.

\subsubsection{Marco teórico}

Se supone la siguiente función de resultados educativos:

$$
y=g(X, e, \theta)
$$

donde $y$ es el desempeño educativo de los alumnos, $X$ es un conjunto de variables de circunstancias, $e$ es el esfuerzo del alumno y $\theta$ es una variable aleatoria (asociada a la suerte del individuo).

Formalmente, existirá IOP en sentido fuerte si dos grupos de alumnos que solamente difieren en sus circunstancias, presentan las mismas probabilidades de obtener los diferentes resultados educativos $y$. Esto es,

Definición 1. Existe IOP en sentido fuerte (IOP-F) si y solo si:

$$
\forall\left(X, X^{\prime}\right) \forall e \forall \theta, F(\cdot \mid X, e, \theta)=F\left(\cdot \mid X^{\prime}, e, \theta\right),
$$

donde $F$ es la función de distribución acumulada.

Sin embargo, esta definición es bastante restrictiva ya que en la realidad es poco probable encontrar dos funciones de distribución acumuladas exactamente iguales. Con lo cual, esta definición no brinda un margen para actuar empíricamente. Una alternativa es trabajar con definiciones más débiles de IOP. Para ello es necesario tener en cuenta criterios que permitan comparar y ordenar las funciones de distribuciones acumuladas según alguna relación de preferencia. Uno de esos criterios es el criterio de dominancia estocástica de primer orden (DEPO) que permite inferir si una distribución es preferida a la otra. ${ }^{2}$

Si se suponen dos distribuciones $\mathrm{A}$ y $\mathrm{B}$, si $F_{A}$ domina estrictamente a $F_{B}$ en sentido estocástico de primer orden entonces $F_{A}<F_{B}$ para todo $y$. Se puede observar de inmediato que $F_{A} \neq F_{B}$ y no se cumple con la definición de IOP-F sugiriendo que entre $\mathrm{A}$ y $\mathrm{B}$ no existe IOP. Sin embargo, la ausencia de dominancia estocástica de primer orden, no brinda un criterio adecuado para asegurar IOP. Por ejemplo, si tenemos dos distribuciones acumuladas, $F_{A}$ y $F_{B}$ cuyas curvas se cortan entre sí de tal manera que A no domina a $\mathrm{B}$ y viceversa, en este caso no habría DEPO; a pesar de esto no sería posible asegurar algo sobre IOP porque podrían darse cualquiera de las dos opciones (que hubiera o no). Podría suceder que la distribución de alumnos del grupo A domine a la distribución de alumnos del grupo B hasta

\footnotetext{
${ }^{1}$ Para un análisis más detallado de la suerte en la teoría de igualdad de oportunidades ver Lefranc et al. (2009).

${ }^{2}$ Sean $F_{A}$ y $F_{B}$ dos distribuciones de probabilidad acumulada, $F_{A}$ DEPO a $F_{B}$ si y solo si A es al menos tan preferida como B bajo cualquier función de utilidad no decreciente $(u)$; esto es, $\int u(y) d F_{A} \geq \int u(y) d F_{B}$. Si la dominancia es estricta entonces A es estrictamente preferida a B.
} 
el resultado y más alto y para el resultado más alto sean los alumnos con más suerte del grupo $\mathrm{B}$ los que presenten más probabilidades de obtener al menos dicho resultado respecto de los alumnos del grupo A. Es decir, en un primer momento los alumnos del grupo A tienen más ventajas sobre los alumnos del grupo B, y luego son los alumnos con más suerte del grupo B los que presentan más ventajas respecto de los demás. Si la suerte aún no está repartida entre los alumnos, la relación de preferencia entre las distribuciones no resulta obvia. En algunos casos podrían ser indiferentes y en otros no. Es así que necesitamos un criterio que considere este tipo de situaciones e incluya la noción de riesgo de las distribuciones. Este criterio es el criterio de dominancia estocástica de segundo orden (DESO). Suponiendo aversión al riesgo, la distribución A DESO a la distribución B, si A es menos riesgosa y las ventajas son al menos tan buenas que en $\mathrm{B}$ (valor esperado). ${ }^{3,4}$

Lefranc et al. (2009), proponen tres definiciones de igualdad de oportunidades más débiles que IOP-F basándose en la dominancia estocástica de segundo orden (DESO). Siguiendo estas definiciones se establece que:

Definición 2. IOP-D1 La igualdad de oportunidades débil bajo aversión al riesgo se cumple si y solo si:

$$
\forall X \neq X^{\prime} \forall e \forall \theta, F(\cdot \mid X, e, \theta) \nsucc_{D E S O} F\left(\cdot \mid X^{\prime}, e, \theta\right) .
$$

Esta definición indica que habrá IOP cuando cualquiera sea el esfuerzo realizado o cualquiera sea $\theta$, no sea posible ordenar las ventajas ofrecidas por los distintos grupos siguiendo el criterio de dominancia estocástica de segundo orden. Esta definición de IOP ofrece reglas de decisión en el caso donde para algún nivel de esfuerzo y/o suerte un grupo provee ventajas sobre los otros y viceversa.

Definición 3. IOP-D2 La igualdad de oportunidades (más) débil se cumple si y solo si:

$$
\begin{gathered}
\nexists\left(X, X^{\prime}\right) \text { tal que } \forall e \forall \theta, F(\cdot \mid X, e, \theta) \succeq_{D E S O} F\left(\cdot \mid X^{\prime}, e, \theta\right) \mathrm{y} \text {, además, } \\
\exists e \text { tal que: } F(\cdot \mid X, e, \theta) \succ_{D E S O} F\left(\cdot \mid X^{\prime}, e, \theta\right) \mathrm{y} / \mathrm{o} \\
\exists \theta \text { tal que: } F(\cdot \mid X, e, \theta) \succ_{D E S O} F\left(\cdot \mid X^{\prime}, e, \theta\right) .
\end{gathered}
$$

La segunda definición de igualdad de oportunidades en sentido débil (IOP-D2) es menos restrictiva que la anterior ya que permite que exista un esfuerzo y/o suerte para el cual uno de los grupos presente ventajas sobre el otro. La idea es que el mismo grupo con ventajas para dicho esfuerzo y/o suerte corra con desventaja por lo menos para algún otro esfuerzo y/o suerte. De esta manera, mientras que el esfuerzo no sea escogido y la suerte realizada ningún grupo será preferido a otro.

Ahora bien en la práctica no se observan completamente las circunstancias sino que se observan parcialmente; por ello es que se brinda una tercera definición más débil que tiene en cuenta solo las circunstancias observadas, $X_{1}$.

\footnotetext{
${ }^{3}$ Asumiendo que el decisor maximizador es averso al riesgo, $F_{A}$ DESO a $F_{B}$ si y solo si $\mathrm{A}$ es al menos tan preferida como B bajo cualquier función de utilidad no decreciente cóncava $(u)$. En el caso particular de que las dos funciones de distribución acumuladas presenten el mismo valor esperado, $\int y d F_{A}=\int y d F_{B} \mathrm{y}$ que $\int u(y) d F_{A} \geq \int u(y) d F_{B}$ entonces se tiene que $F_{A}$ DESO a $F_{B}$. Si la dominancia es estricta entonces A es estrictamente preferida a B.

${ }^{4}$ En este estudio, la noción de riesgo es asociada a las desigualdades dentro de cada grupo proveniente de la influencia conjunta de la suerte y otras variables de esfuerzo o circunstancias no observadas.
} 
Definición 4. IOP-D3 Cuando las circunstancias son parcialmente observadas, la igualdad de oportunidades (más) débil se define como:

$$
\begin{gathered}
\nexists\left(X_{1}, X_{1}^{\prime}\right) \text { tal que: } \forall\left(X_{2}, X_{2}^{\prime}\right), \forall e, \forall \theta, \\
F\left(\cdot \mid X_{1}, X_{2}, e, \theta\right) \succ_{D E S O} F\left(\cdot \mid X_{1}^{\prime}, X_{2}^{\prime}, e, \theta\right)
\end{gathered}
$$

donde $X_{1}$ son las circunstancias parcialmente observadas y $X_{2}$ son las no observadas.

\subsubsection{Condiciones necesarias y suficientes para la existencia de IOP}

Para proveer condiciones necesarias y/o suficientes para la existencia de IOP, es preciso plantear algunos criterios de aplicación. Lefranc et al. (2009) proponen criterios de implementación (CI) para dos situaciones: (1) cuando las circunstancias son observadas completamente y el esfuerzo no es observado y (2) cuando las circunstancias son parcialmente observadas y el esfuerzo no es observado.

1. Circunstancias $(X)$ observadas, esfuerzo (e) y $\theta$ no observados.

CI1: El primer criterio de aplicación para IOP es que las distribuciones condicionadas sean iguales. Es decir, CI1 se satisface si y solo si

$$
\forall\left(X, X^{\prime}\right), F(. \mid X)=F\left(. \mid X^{\prime}\right) .
$$

Si $e$ y $\theta$ son independientes de $X$ entonces: IOP-F $\Rightarrow$ CI1. Esto es, CI1 es una condición necesaria para IOP-F.

CI2: El segundo criterio de aplicación se cumple si y solo si:

$$
\forall X \neq X^{\prime}, F(. \mid X) \nsucc_{D E S O} F\left(. \mid X^{\prime}\right) .
$$

Si e y $\theta$ son independientes de $X$ entonces CI2 es una condición suficiente para IOP-D2.

2. Circunstancias $(X)$ observadas parcialmente, esfuerzo (e) y $\theta$ no observados.

Si e y $\theta$ son independientes de $X$ entonces CI2 es condición suficiente para IOP-D3.

En este trabajo contemplamos una tercera situación donde el esfuerzo y las circunstancias son parcialmente observados obteniendo así los siguientes criterios, adicionales a los anteriores ya conocidos:

3. Circunstancias $(X)$ y esfuerzo (e) observados parcialmente y $\theta$ no observado.

CI1bis: El criterio se satisface si y solo si:

$$
\forall\left(X_{1}, X_{1}^{\prime}\right), F\left(. \mid X_{1}, e_{1}\right)=F\left(. \mid X_{1}^{\prime}, e_{1}\right)
$$

donde $e=\left(e_{1}, e_{2}\right), e_{1}$ es observado y $e_{2}$ no es observado, $X=\left(X_{1}, X_{2}\right), X_{1}$ se observa y $X_{2}$ no se observa.

Si $\theta$ es independiente de $X$ y $e$; el esfuerzo no observado $e_{2}$ es independiente de $e_{1}$ y $X$ y, además, el esfuerzo observado $e_{1}$ es independiente de $X_{2}$ entonces IOP-F $\Rightarrow$ CI1bis. 
Esto es, CI1bis es una condición necesaria para IOP-F.

\section{Demostración:}

Si IOP-F se satisface tenemos que $\forall\left(X_{1}, X_{1}^{\prime}\right), \forall\left(X_{2}, X_{2}^{\prime}\right), \forall e_{1}, e_{2}, \forall \theta$ y $\forall y$

$$
F\left(y \mid X_{1}, X_{2}, e_{1}, e_{2}, \theta\right)=F\left(y \mid X_{1}^{\prime}, X_{2}^{\prime}, e_{1}, e_{2}, \theta\right) .
$$

Esto es:

$$
F(y \mid X, e, \theta)=F\left(y \mid X^{\prime}, e, \theta\right) .
$$

Si $\forall X, \forall e, H(\theta \mid e, X)=H(\theta)$ entonces a partir de IOP-F

$$
\begin{aligned}
& P(y \leqslant y \mid X=\bar{X}, e=\bar{e}, \theta=\bar{\theta})=P\left(y \leqslant y \mid X^{\prime}=\bar{X}^{\prime}, e=\bar{e}, \theta=\bar{\theta}\right) \\
& \frac{P(y \leqslant y, X=\bar{X}, e=\bar{e}, \theta=\bar{\theta})}{P(X=\bar{X}, e=\bar{e}) P(\theta=\bar{\theta})}=\frac{P\left(y \leqslant y, X^{\prime}=\bar{X}^{\prime}, e=\bar{e}, \theta=\bar{\theta}\right)}{P\left(X^{\prime}=\bar{X}^{\prime}, e=\bar{e}\right) P(\theta=\bar{\theta})} \\
& \frac{P(y \leqslant y, X=\bar{X}, e=\bar{e}, \theta=\bar{\theta})}{P(X=\bar{X}, e=\bar{e})}=\frac{P\left(y \leqslant y, X^{\prime}=\bar{X}^{\prime}, e=\bar{e}, \theta=\bar{\theta}\right)}{P\left(X^{\prime}=\bar{X}^{\prime}, e=\bar{e}\right)} \\
& P(y \leqslant y, \theta=\bar{\theta} \mid X=\bar{X}, e=\bar{e})=P\left(y \leqslant y, \theta=\bar{\theta} \mid X^{\prime}=\bar{X}^{\prime}, e=\bar{e}\right)
\end{aligned}
$$

e integrando sobre los valores de $\bar{\theta}$ :

$$
\begin{aligned}
\int P(y \leqslant y, \theta=\bar{\theta} \mid X=\bar{X}, e=\bar{e}) d \bar{\theta} & =\int P\left(y \leqslant y, \theta=\bar{\theta} \mid X^{\prime}=\bar{X}^{\prime}, e=\bar{e}\right) d \bar{\theta} \\
P(y \leqslant y \mid X=\bar{X}, e=\bar{e}) & =P\left(y \leqslant y \mid X^{\prime}=\bar{X}^{\prime}, e=\bar{e}\right)
\end{aligned}
$$

se tiene que: ${ }^{5}$

$$
F(y \mid X, e)=F\left(y \mid X^{\prime}, e\right) .
$$

Esto es,

$$
F\left(y \mid X_{1}, X_{2}, e_{1}, e_{2}\right)=F\left(y \mid X_{1}^{\prime}, X_{2}^{\prime}, e_{1}, e_{2}\right) .
$$

${ }^{5}$ Algo similar se obtiene a partir de las funciones de densidad,

$$
f(y \mid X, e, \theta)=f\left(y \mid X^{\prime}, e, \theta\right) \Rightarrow \frac{f(y, X, e, \theta)}{f(X, e) f(\theta)}=\frac{f\left(y, X^{\prime}, e, \theta\right)}{f\left(X^{\prime}, e\right) f(\theta)} .
$$

Integrando,

$$
\int \frac{f(y, X, e, \theta)}{f(X, e)} d \theta=\int \frac{f\left(y, X^{\prime}, e, \theta\right)}{f\left(X^{\prime}, e\right)} d \theta \Rightarrow \frac{f(y, X, e)}{f(X, e)}=\frac{f\left(y, X^{\prime}, e\right)}{f\left(X^{\prime}, e\right)} \Rightarrow f(y \mid X, e)=f\left(y \mid X^{\prime}, e\right) .
$$


Luego, si $e_{2}$ es independiente de $e_{1}$ y las circunstancias $\left(X_{1}, X_{2}\right)$, de manera similar integrando sobre los valores de $e_{2}$ se obtiene que:

$$
\forall\left(X_{1}, X_{1}^{\prime}\right), \forall\left(X_{2}, X_{2}^{\prime}\right), \forall e_{1}, F\left(y \mid X_{1}, X_{2}, e_{1}\right)=F\left(y \mid X_{1}^{\prime}, X_{2}^{\prime}, e_{1}\right) .
$$

Si $X_{2}$ y $X_{2}^{\prime}$ son independientes de $e_{1}$, integrando se obtiene que:

$$
\forall\left(X_{1}, X_{1}^{\prime}\right), \forall e_{1}, F\left(y \mid X_{1}, e_{1}\right)=F\left(y \mid X_{1}^{\prime}, e_{1}\right),
$$

lo cual completa la demostración.

A diferencia de los otros CI, es posible relajar algunos de los supuestos sobre e y $X$ en el CI1bis. En la demostración no se realizan supuestos sobre la relación entre $e_{1} \mathrm{y}$ $X_{1}$. Con lo cual en este criterio no se necesita que el esfuerzo observado del alumno pueda o no estar relacionado con las circunstancias observadas. En la práctica, esto resulta ventajoso ya que algunas variables proxy de esfuerzo como el tiempo de estudio pueden estar asociadas en parte con el entorno del alumno observado. Por otra parte, se supone que $e_{2}$ son variables que dependen estrictamente del alumno, es decir, que son controlables por él y no depende de otras circunstancias o decisiones. En cuanto al supuesto de independencia de $\theta$, en el modelo se plantea que este término refleja la aleatoriedad de los resultados (la suerte de los alumnos) cuestión que, en principio, no se contrapone con el supuesto de independencia.

En cuanto a CI2, se propone el siguiente criterio alternativo cuando las circunstancias y el esfuerzo son observados parcialmente:

CI2bis: El criterio de implementación se cumple si y solo si:

$$
\forall X_{1} \neq X_{1}^{\prime}, F\left(. \mid X_{1}, e_{1}\right) \nsucc_{D E S O} F\left(. \mid X_{1}^{\prime}, e_{1}\right) \text {. }
$$

Si $\theta$ es independiente de $X$ y $e$; y $X_{2}$ y $e_{2}$ son independientes de $X_{1}, e_{1}$ entonces CI2bis es una condición suficiente para IOP-D3.

\section{Demostración:}

Si negamos IOP-D3 entonces $\exists\left(X_{1}, X_{1}^{\prime}\right)$ tal que: $\forall\left(X_{2}, X_{2}^{\prime}\right), \forall\left(e_{1}, e_{2}\right), \forall \theta$,

$$
F\left(\cdot \mid X_{1}, X_{2}, e_{1}, e_{2}, \theta\right) \succ_{D E S O} F\left(\cdot \mid X_{1}^{\prime}, X_{2}^{\prime}, e_{1}, e_{2}, \theta\right) .
$$

En particular si se considera que $X_{2}=X_{2}^{\prime}$, integrando la relación de dominancia sobre $\theta$ y dado que $\theta$ es independiente de $X$ y $e$ :

$$
F\left(\cdot \mid X_{1}, X_{2}, e_{1}, e_{2}\right) \succ_{D E S O} F\left(\cdot \mid X_{1}^{\prime}, X_{2}^{\prime}, e_{1}, e_{2}\right)
$$

e integrando sobre $e_{2}$ y $X_{2}$ dado que son independientes de $X_{1}$ y $e_{1}$ :

$$
F\left(\cdot \mid X_{1}, e_{1}\right) \succ_{D E S O} F\left(\cdot \mid X_{1}^{\prime}, e_{1}\right)
$$

entonces CI2bis no se cumple. 


\subsubsection{Test de igualdad de oportunidades}

Una vez establecidas las condiciones necesarias y las condiciones suficientes para la existencia de IOP se prueba para este problema cuáles de ellas se satisfacen y cuáles no. El objetivo es llevar a cabo una serie de tests que brinden información si entre dos grupos de alumnos que realizan un esfuerzo similar y que difieren en circunstancias presentan condiciones para IOP.

En este caso las hipótesis nulas a testear son:

(1) $H_{0}$ : Las distribuciones correspondiente a los grupos $\left(X_{1}, e_{1}\right)$ y $\left(X_{1}^{\prime}, e_{1}\right)$ son iguales. Es decir, $F\left(. \mid X_{1}, e_{1}\right)=F\left(. \mid X_{1}^{\prime}, e_{1}\right)$.

(2) $H_{0}$ : La distribución del grupo $\left(X_{1}, e_{1}\right)$ domina estocásticamente en primer orden a la distribución del grupo $\left(X_{1}^{\prime}, e_{1}\right)$. Es decir, $F\left(. \mid X_{1}, e_{1}\right) \succ_{D E P O} F\left(. \mid X_{1}^{\prime}, e_{1}\right)$.

(3) $H_{0}$ : La distribución del grupo $\left(X_{1}^{\prime}, e_{1}\right)$ domina estocásticamente en primer orden a la distribución del grupo $\left(X_{1}, e_{1}\right)$. Es decir, $F\left(. \mid X_{1}^{\prime}, e_{1}\right) \succ_{D E P O} F\left(. \mid X_{1}, e_{1}\right)$.

(4) $H_{0}$ : La distribución del grupo $\left(X_{1}, e_{1}\right)$ domina estocásticamente en segundo orden a la distribución del grupo $\left(X_{1}^{\prime}, e_{1}\right)$. Es decir, $F\left(. \mid X_{1}, e_{1}\right) \succ_{D E S O} F\left(. \mid X_{1}^{\prime}, e_{1}\right)$.

(5) $H_{0}$ : La distribución del grupo $\left(X_{1}^{\prime}, e_{1}\right)$ domina estocásticamente en segundo orden a la distribución del grupo $\left(X_{1}, e_{1}\right)$. Es decir, $F\left(. \mid X_{1}^{\prime}, e_{1}\right) \succ_{D E S O} F\left(. \mid X_{1}, e_{1}\right)$.

Entonces, un procedimiento a seguir es:

a. Si no se rechaza la hipótesis nula del test (1), se asume que se satisface el CI1bis y se cumple con la condición necesaria para que exista igualdad de oportunidades.

b. Si se rechaza la hipótesis nula del test (1), no se satisface el CI1bis y se prueban las restantes hipótesis:

b.1 Si los test (2) y (3) sugieren dominancia estocástica estricta de primer orden de una distribución sobre la otra, por ejemplo:

$$
F\left(. \mid X_{1}, e_{1}\right) \succ_{D E P O} F\left(. \mid X_{1}^{\prime}, e_{1}\right) \text { y } F\left(. \mid X_{1}^{\prime}, e_{1}\right) \nsucceq_{D E P O} F\left(. \mid X_{1}, e_{1}\right),
$$

no se cumple con los criterios CI1bis y CI2bis lo que sugiere que no existe igualdad de oportunidades al no cumplirse ninguna de las condiciones tanto necesarias como suficientes. Si estos test no sugiriesen dominancia entonces se prueban las hipótesis (4) y (5).

b.2 Si los test (4) y (5) sugieren dominancia estocástica estricta de segundo orden de una distribución sobre la otra, por ejemplo:

$$
F\left(. \mid X_{1}, e_{1}\right) \succ_{D E S O} F\left(. \mid X_{1}^{\prime}, e_{1}\right) \text { y } F\left(. \mid X_{1}^{\prime}, e_{1}\right) \nsucceq_{D E S O} F\left(. \mid X_{1}, e_{1}\right),
$$

no se cumple con los criterios CI1bis y CI2bis. No se cumplen ni las condiciones necesarias ni las suficientes para igualdad de oportunidades.

b.3 Si a partir de los test (4) y (5) se rechaza la dominancia de cada distribución sobre la otra

$$
F\left(. \mid X_{1}, e_{1}\right) \nsucc_{D E S O} F\left(. \mid X_{1}^{\prime}, e_{1}\right) \text { y } F\left(. \mid X_{1}^{\prime}, e_{1}\right) \nsucc_{D E S O} F\left(. \mid X_{1}, e_{1}\right),
$$

el CI2bis se satisface pero no el CI1bis, de esta manera se cumple con la condición suficiente para que exista igualdad de oportunidades en sentido débil. 
b.4 Si los test (4) y (5) sugieren que ambas distribuciones se dominan entre sí,

$$
F\left(. \mid X_{1}, e_{1}\right) \succeq_{D E S O} F\left(. \mid X_{1}^{\prime}, e_{1}\right) \text { y } F\left(. \mid X_{1}^{\prime}, e_{1}\right) \succeq_{D E S O} F\left(. \mid X_{1}, e_{1}\right)
$$

y teniendo en cuenta el test (1) se tiene que el CI2bis se satisface pero no el CI1bis, es decir, esta situación es condición suficiente para que haya igualdad de oportunidades en sentido débil.

Cabe señalar que los resultados de las pruebas de hipótesis tienen que ser coherentes entre sí teniendo en cuenta que DEPO implica DESO pero no viceversa. Para poder testear estas hipótesis a partir de los datos, primero, hay que estimar las funciones de distribución del desempeño educativo de cada grupo de alumnos. Siguiendo a Davidson y Duclos (2000), se estiman dichas funciones utilizando distintos umbrales, $z$, y explotando la relación existente entre índices de pobreza y dominancia estocástica hallada por Foster y Shorrocks (1988a,b).

Sea $D_{A}^{1}(z)=F_{A}(z)$ la función de distribución acumulada del grupo A hasta $z$ y $D_{A}^{g}(z)=$ $\int_{0}^{z} D_{A}^{(g-1)}(y) d y$ para valores de $g \geq 2$, Davidson y Duclos (2000) proponen expresar dichas funciones de la siguiente manera:

$$
D^{g}(z)=\frac{1}{(g-1) !} \int_{0}^{z}(z-y)^{g-1} d y
$$

De esta forma la distribución $\mathrm{B}$ domina a la distribución A en el sentido de dominancia estocástica de orden $g$, si $D_{A}^{g}(z) \geq D_{B}^{g}(z)$ para todo $z \in \Re$. En particular, los autores señalan que los umbrales $z$ tienen que ser exógenos.

Para una muestra de tamaño $\mathrm{N}$, el estimador de $D^{g}(z)$ es:

$$
\hat{D}^{g}(z)=\frac{1}{N(g-1) !} \sum_{i=1}^{N}\left(z-y_{i}\right)^{g-1} I\left(y_{i} \leq z\right)
$$

donde $I\left(\right.$.) es una función indicadora que toma valor 1 si se cumple que $y_{i} \leq z$. De esta manera, si se consideran $k$ umbrales $\left(z_{1}, z_{2}, \ldots, z_{k}\right)$ se obtiene el vector $\hat{D}^{g}=\left(\hat{D}^{g}\left(z_{1}\right), \hat{D}^{g}\left(z_{2}\right), \ldots, \hat{D}^{g}\left(z_{k}\right)\right)$ y la varianza asintótica $\sum_{D}$. Específicamente, en este estudio se consideran 99 umbrales, es decir un $k=99$. Con el fin de que los $k$ umbrales sean exógenos se calcula el máximo valor teórico que puede tomar la variable de desempeño menos el mínimo valor teórico y se divide dicho valor por $100 .{ }^{6}$ Luego, para comparar las distribuciones A y B se utiliza el siguiente estimador

$$
\hat{\delta}=\left(\hat{D}_{B}^{g}-\hat{D}_{A}^{g}\right)
$$

cuya matriz de varianza y covarianza es $\sum=\frac{\sum_{A}}{N_{A}}+\frac{\sum_{B}}{N_{B}}$ (se asume independencia entre las distribuciones de A y B). Si las distribuciones son iguales entonces $\hat{\delta}=0$. Para probar que ambas distribuciones son iguales se realiza el test de Wald cuya hipótesis nula es $H_{0}: \delta=0$ y la hipótesis alternativa es $H_{1}: \delta \neq 0$. El estadístico $T_{1}$ asociado a dicho test sigue una distribución chi-cuadrado con $k$ grados de libertad:

$$
T_{1}=\hat{\delta}^{\prime}\left(\frac{\sum_{A}}{N_{A}}+\frac{\sum_{B}}{N_{B}}\right)^{-1} \hat{\delta} \sim \chi_{k}^{2}
$$

\footnotetext{
${ }^{6}$ En este caso, la variable son los puntajes de las pruebas PISA y se toma un valor máximo de 800 y un valor mínimo de 0 , de esta manera se tiene en cuenta aproximadamente 3 -sigmas de la distribución de los puntajes.
} 
En el caso de dominancia estocástica, la hipótesis a testear es $H_{0}: \delta \in \Re_{+}^{k}$ y la hipótesis alternativa $H_{1}: \delta \notin \Re_{+}^{k}$. Para este test utilizamos el siguiente estadístico desarrollado por Kodde y Palm (1986),

$$
T_{2}=\min _{\delta \in \Re_{+}^{k}}\|\hat{\delta}-\delta\| \sim \bar{\chi}^{2}
$$

donde $\|\hat{\delta}-\delta\|=(\hat{\delta}-\delta)^{\prime} \sum^{-1}(\hat{\delta}-\delta)$ y $M^{\prime}$ es la matriz transpuesta de $M$ y para obtener el valor de $T_{2}$ se procede a resolver el problema de minimización. El estadístico sigue una distribución mixta de chi-cuadrado,

$$
\bar{\chi}^{2}=\sum_{j=0}^{k} \omega\left(k, j, \sum\right) \operatorname{Pr}\left(\chi_{j}^{2} \geqslant c\right)
$$

donde $\omega\left(k, j, \sum\right)$ es la probabilidad de que $j$ elementos sean estrictamente positivos, $j \in(0, k]$ y $c$ es un valor crítico de la distribución. Si bien Kodde y Palm (1986) proponen límites inferiores y superiores de varios valores críticos, la distribución de chi-cuadrado mixta no tiene una tabulación estandarizada para todos los valores. En los casos donde no es posible llegar a una conclusión a partir de dichos límites se sigue a Lefranc et al. (2009) y se estima el pvalor del estadístico $T_{2}$ a partir de simulaciones de Monte Carlo. Para ello se simulan 10.0000 vectores multivariados independientes normalmente distribuidos con media 0 y matriz de varianza-covarianza $\sum$. A partir de los mismos se calcula cuántos de ellos tienen $j$ elementos estrictamente positivos $(j=1, \ldots, k)$ y se obtiene $\omega_{j}$. Luego se computa el p-valor que permite rechazar o no la hipótesis nula.

Finalmente, para concluir si una distribución domina a la otra se realizan dos test. Primero, se prueba si la distribución A domina a la distribución B y luego se testea si B domina a A. Si no se rechaza que A domina a $B$ y se rechaza que B domina a A, entonces se dirá que A domina a B. Si no se rechaza que A domina a B y tampoco se rechaza que B domina a A, entonces se deduce que A y B son iguales. Si se rechaza que A domina a B y no se rechaza que $\mathrm{B}$ domina a $\mathrm{A}$, entonces $\mathrm{B}$ domina a $\mathrm{A}$. Si se rechaza que $\mathrm{A}$ domina a $\mathrm{B}$ y se rechaza también que B domina a A, entonces se infiere que las curvas de distribución se cruzan. En los casos donde se concluye que una distribución domina a la otra pero no viceversa, son aquellos casos donde no hay igualdad de oportunidades.

\subsection{Definición de variables y datos}

En este trabajo se utiliza la base de datos del Programa para la Evaluación Internacional de los Estudiantes (PISA) de la OCDE de los años 2006, 2009 y 2012. Como ya se ha mencionado, el programa PISA es un estudio de evaluación de los resultados de los sistemas educativos y brinda información representativa de los alumnos entre 15 años y 3 meses y 16 años y 2 meses que han terminado al menos 6 cursos de enseñanza obligatoria. PISA se centra en medir la capacidad de los jóvenes para usar su conocimiento y sus destrezas en la vida diaria; enfocándose en las competencias científica, lectora y matemática de los estudiantes más que en el dominio de la currícula escolar.

Las variables de resultado educativo son los puntajes de las pruebas estandarizadas de matemáticas, lectura y ciencia, cada una por separado. Estos puntajes ofrecen información sobre el desempeño de los alumnos, este último considerado en sentido amplio no solo conocimientos sino también habilidades. Mientras más altos son los puntajes mayores son las competencias de los estudiantes.

En cuanto a las circunstancias, la literatura indica una fuerte relación entre el entorno del alumno y su desempeño educativo (Hanushek, 2006); mientras que señala una asociación menor con los recursos de las escuelas como pueden ser el gasto educativo per cápita, 
la cantidad de computadoras, el número de profesores, características del aula, etc. (Hanushek, 1979, 1986, 1997; Betts, 1996; Case y Deaton, 1999; Angrist y Lavy, 1999). Esta evidencia empírica hace que sea relevante incluir al análisis público-privado además de las características del colegio, el contexto familiar y educativo como circunstancias del alumno. ${ }^{7}$

Las variables de circunstancias, $X_{1}$, que se consideran son el tipo de establecimiento, el clima educativo del colegio, el nivel educativo máximo alcanzado por los padres y el nivel socioeconómico cultural familiar. Las primeras dos son variables a nivel escuela mientras que las últimas dos son variables individuales de los alumnos. El tipo de establecimiento puede ser público, privado subvencionado o privado independiente. Los establecimientos privados independientes se caracterizan por ser de gestión privada y no recibir ningún fondo del Estado. Los establecimientos privados subvencionados si bien son de gestión privada reciben fondos del Estado. Por otro lado, los establecimientos públicos son de gestión estatal. El clima educativo del colegio se mide a partir del nivel educativo promedio de los padres del colegio. El clima educativo puede ser alto si los padres del colegio tienen, en promedio, estudios superiores. Un clima educativo medio corresponde al caso donde los padres poseen, en promedio, estudios secundarios. Un clima educativo bajo corresponde a si los padres, en promedio, tienen solo estudios primarios.

El nivel educativo de los padres también puede ser alto, medio o bajo, es alto cuando alguno de los dos padres tiene estudios superiores, medio cuando el máximo nivel alcanzado corresponde a estudios secundarios y bajo cuando solo han alcanzado los estudios primarios. El nivel socioeconómico cultural de la familia se construye a partir del índice ESCS (economic, social and cultural status, por sus siglas en inglés) que ofrece PISA el cual tiene en cuenta no solo el estatus ocupacional de los padres, los años de educación de los padres, la riqueza del hogar, los bienes y servicios del hogar sino también el nivel cultural familiar a partir de los libros que poseen (ya sean clásicos o de poesía) y la posesión de obras de arte como por ejemplo cuadros. El nivel socioeconómico puede ser alto, medio o bajo, para ello se divide la distribución del índice ESCS en terciles representando cada categoría un tercio de la distribución.

Las escuelas en el país, ya sean públicas o privadas, son bastante heterogéneas. Con el objetivo de ahondar en estas heterogeneidades propias de cada uno de los tipos de establecimientos educativos es que se incorporan al análisis dos variables de circunstancias asociadas a la escuela: la calidad de los recursos educativos del colegio y la calidad de los profesores. La calidad de los recursos del colegio se define a partir del índice SCMATEDU y al igual que con el índice de nivel socioeconómico se divide a la distribución en tres partes iguales, clasificando a la calidad de los recursos educativos en alta, media o baja. En el caso de la calidad de los profesores, se arma un índice teniendo en cuenta cuatro variables 1) la relación entre profesores con dedicación full-time y profesores con dedicación part-time de la escuela, 2) la cantidad de estudiantes por profesor, 3) la proporción de profesores certificados y 4) la proporción de profesores con estudios universitarios. El índice puede tomar valores entre 0 y 1 , y se obtiene como una proporción de los cuatro ítems anteriores. Se analiza, para cada una de las variables, si el colegio muestra un valor superior o inferior al de la mediana. ${ }^{8}$

\footnotetext{
${ }^{7}$ El famoso reporte de Coleman et al. (1966) fue uno de los primeros estudios en mostrar que el factor más importante en la relación entre el desempeño educativo y la calidad de la escuela no era la escuela en sí sino el contexto familiar del alumno y el efecto de pares en la escuela, a partir del mismo surgieron una gran cantidad de estudios enfocados en el análisis de estas relaciones.

${ }^{8} \mathrm{Si}$ el índice de calidad de los profesores toma valor 1 es porque el ratio de profesores con dedicación full-time y dedicación part-time, la proporción de profesores certificados y la proporción de profesores con estudios universitarios del establecimiento son superiores o iguales a la mediana de cada uno de ellos, y también la cantidad de estudiantes por profesor es menor a la mediana. Si toma el valor 0.75 es que el colegio tiene ventaja respecto de la mediana en tres de los ítems, 0.50 solo en dos de los ítems, 0.25 solo en
} 
Finalmente, la calidad de los profesores puede ser alta, media o baja dependiendo de los terciles del índice computado.

En cuanto al esfuerzo, si bien es parte de la teoría de IOP generalmente en el análisis empírico se excluye (por ejemplo Lefranc et al., 2009). Esto se debe, principalmente, a que es un concepto subjetivo con limitaciones en su medición y son pocas las bases de datos que contienen variables proxy de esfuerzo individual. En el año 2012, el cuestionario de PISA incorporó algunas preguntas específicas sobre la cantidad de tiempo que dedica el alumno a la realización de tareas escolares de matemáticas, lectura y ciencia fuera del colegio. Estas horas de estudio conllevan un costo de oportunidad (menos horas de ocio) para el alumno implicando algún tipo de esfuerzo individual. Por esta razón, se puede considerar la cantidad de horas de estudio de los alumnos fuera del colegio como una variable de esfuerzo $(e)$. Esta variable toma valor 1 si el alumno dedica más de 2 horas por semana y 0 si el alumno dedica 2 horas o menos por semana. El primer caso corresponde a un esfuerzo alto y el segundo a un esfuerzo bajo. No obstante, no se dispone de esta variable para los años 2006 y 2009.

Cuando se trabaja con los años 2006, 2009 y 2012 en forma conjunta, no se cuenta con la cantidad de horas de estudio. Como alternativa se considera como variable de esfuerzo la cantidad de horas de clases de matemáticas, lectura y ciencia por semana que un alumno tiene en la escuela (se toma las horas de cada materia en forma separada). Si bien la cantidad de horas de clase no corresponde estrictamente a un esfuerzo por parte del alumno, resulta conveniente comparar grupos de alumnos que tienen similares cargas horarias. La idea principal es que la desigualdad que se genera a partir de los distintos esfuerzos es considerada socialmente aceptable. En un principio, también se podría considerar la desigualdad proveniente de diferentes cargas horarias como socialmente aceptable. En el caso que los estudiantes tengan más de 6 horas de clases en una determinada materia (ya sea matemática, lengua o ciencia) el esfuerzo en dicho desempeño será considerado como alto y en el caso que las horas de clases por semana sean 6 horas o menos el esfuerzo será considerado como bajo.

A partir de las diferentes combinaciones de las variables es posible armar varios grupos de alumnos. Primero se dividen a los alumnos en tres grupos 1) alumnos que asisten a la escuela pública, 2) alumnos que asisten a la escuela privada subvencionada y 3) alumnos que asisten a la escuela privada independiente. En segundo lugar se combina el tipo de establecimiento con el nivel de esfuerzo y se obtienen 6 grupos, 1) alumnos que asisten a la escuela pública y realizan esfuerzo alto, 2) alumnos que asisten a la privada subvencionada y realizan esfuerzo alto, 3) alumnos que asisten a la escuela privada independiente y realizan esfuerzo alto, 4) alumnos que asisten a la escuela pública y realizan esfuerzo bajo, 5) alumnos que asisten a la privada subvencionada y realizan esfuerzo bajo, 6) alumnos que asisten a la escuela privada independiente y realizan esfuerzo bajo. En tercer lugar se tiene en cuenta el tipo de establecimiento, el nivel de esfuerzo y el nivel educativo de los padres (alto, medio y bajo) obteniendo 18 grupos. En una cuarta situación se combina el tipo de establecimiento, el nivel de esfuerzo y el nivel socioeconómico cultural de la familia, obteniendo también 18 grupos. A su vez, se combina el tipo de establecimiento, el nivel de esfuerzo con el clima educativo del colegio generando 18 grupos. También se combina el tipo de establecimiento, el nivel de esfuerzo y la calidad de los recursos educativos del colegio obteniendo 18 grupos. Por último, también se forman 18 grupos a partir del tipo de establecimiento, el esfuerzo del alumno y la calidad de los profesores del colegio.

Debido a que el número de grupos cuando se consideran tres variables asciende a 18 y la cantidad de observaciones de cada uno de los grupos se reduce considerablemente, es que se decide trabajar con solo dos circunstancias a la vez y se utiliza una muestra que contiene observaciones de los años 2006, 2009 y 2012. La cantidad total de observaciones de la muestra

un item y 0 si el colegio no presenta ventaja en ninguno de los ítems considerados. 
al considerar los tres años, 2006, 2009 y 2012, es aproximadamente $14.500 .^{9}$ La Tabla 3.1 muestra que el $68 \%$ de los alumnos asisten a escuelas públicas, el $23 \%$ a escuelas privadas subvencionadas y el $9 \%$ a escuelas privadas independientes. A pesar del considerable tamaño de la muestra, al dividir según las circunstancias la cantidad de observaciones del grupo de privadas independientes con nivel bajo se reduce notablemente, de allí que las conclusiones respecto a este tipo de grupo se tengan que realizar con cautela.

Tabla 3.1: Cantidad de observaciones según grupos de alumnos.

\begin{tabular}{|c|c|c|c|c|}
\hline \multirow{2}{*}{$\begin{array}{l}\text { Cantidad de } \\
\text { observaciones }\end{array}$} & \multicolumn{4}{|c|}{ Tipo de establecimiento } \\
\hline & Público & Privado subvencionado & Privado independiente & Total \\
\hline \multicolumn{5}{|l|}{ Muestra por año } \\
\hline 2006 & 2983 & 1002 & 252 & 4237 \\
\hline 2009 & 3229 & 940 & 534 & 4703 \\
\hline 2012 & 3753 & 1494 & 472 & 5719 \\
\hline Muestra total & 9965 & 3436 & 1258 & 14659 \\
\hline \multicolumn{5}{|c|}{ Según máximo nivel educativo alcanzado por los padres } \\
\hline Alto & 3844 & 2033 & 869 & 6746 \\
\hline Medio & 3366 & 1004 & 267 & 4637 \\
\hline Bajo & 2413 & 331 & 95 & 2839 \\
\hline Muestra total & 9623 & 3368 & 1231 & 14222 \\
\hline \multicolumn{5}{|c|}{ Según estatus socioeconómico cultural de la familia } \\
\hline Alto & 2,329 & 1,765 & 823 & 4,917 \\
\hline Medio & 3,434 & 1,080 & 271 & 4,785 \\
\hline Bajo & 4,036 & 540 & 154 & 4,730 \\
\hline Muestra total & 9,799 & 3,385 & 1,248 & 14,432 \\
\hline \multicolumn{5}{|c|}{ Según clima educativo del colegio } \\
\hline Alto & 1,253 & 1,947 & 866 & 4,066 \\
\hline Medio & 8,303 & 1,431 & 385 & 10,119 \\
\hline Bajo & 407 & 58 & s.d. & 465 \\
\hline Muestra total & 9,963 & 3,436 & 1,251 & 14,650 \\
\hline \multicolumn{5}{|c|}{ Según calidad recursos educativos del colegio } \\
\hline Alto & 2,495 & 1,497 & 607 & 4,599 \\
\hline Medio & 3,703 & 1,123 & 425 & 5,251 \\
\hline Bajo & 3,725 & 816 & 226 & 4,541 \\
\hline Muestra total & 9,923 & 3,436 & 1,258 & 14,391 \\
\hline \multicolumn{5}{|c|}{ Según calidad de los profesores del colegio } \\
\hline Alto & 660 & 394 & 87 & 1,141 \\
\hline Medio & 1,268 & 675 & 197 & 2,140 \\
\hline Bajo & 2,559 & 901 & 270 & 3,460 \\
\hline Muestra total & 4,487 & 1,970 & 554 & 6,741 \\
\hline
\end{tabular}

Fuente: Elaboración propia en base a datos PISA.

Por otro lado, cuando se dividen a los alumnos según el tipo de establecimiento, se encuentra que el mayor desempeño educativo promedio corresponde al grupo de alumnos que asisten a escuelas privadas independientes (ver Tabla 3.2). A su vez, cuando se comparan grupos de alumnos que se esfuerzan de igual manera, se halla que los alumnos que se esfuerzan alto presentan puntajes promedios superiores a los grupos de alumnos que se esfuerzan bajo (ver Tabla 3.3). También se observa que las diferencias entre escuelas públicas y privadas se mantienen, y las diferencias entre las escuelas privadas subvencionadas e independientes disminuyen. En algunos casos los alumnos que asisten a la escuela subvencionada son los que presentan, en promedio, puntajes más altos respecto a los demás.

${ }^{9}$ El número de observaciones de la muestra del año 2012 asciende a 3.398, de las cuales 2.062 corresponden a alumnos que asisten a escuelas públicas, 931 a escuelas subvencionadas y 288 a escuelas independientes. Si además se divide a los alumnos en aquellos que realizan un esfuerzo bajo y un esfuerzo alto, el grupo de mayor tamaño tiene 1.183 observaciones (pública-esfuerzo bajo) y el grupo con menos observaciones alcanza solo 129 (privada independiente - esfuerzo bajo). 
Tabla 3.2: Puntajes promedios de las pruebas PISA según tipo de establecimiento educativo.

\begin{tabular}{rcccc}
\hline $\begin{array}{c}\text { Pomedio puntajes } \\
\text { pruebas PISA }\end{array}$ & Público & \multicolumn{3}{c}{ Tipo de establecimiento } \\
\hline Muestra total & & & \multirow{2}{*}{ Potal } \\
Matemática & 362 & 429 & 437 & 386 \\
& {$[2.80]$} & {$[4.80]$} & {$[10.15]$} & {$[2.64]$} \\
Lectura & 360 & 442 & 448 & 389 \\
& {$[3.30]$} & {$[5.98]$} & {$[10.83]$} & {$[3.00]$} \\
Ciencia & 373 & 448 & 451 & 399 \\
& {$[2.92]$} & {$[5.12]$} & {$[9.84]$} & {$[2.79]$} \\
\hline
\end{tabular}

Nota: errores estándar entre corchetes.

Fuente: Elaboración propia en base a datos PISA.

Tabla 3.3: Puntajes promedios de las pruebas PISA según tipo de establecimiento educativo y esfuerzo.

\begin{tabular}{|c|c|c|c|c|}
\hline \multirow{2}{*}{$\begin{array}{c}\text { Pomedio puntajes } \\
\text { pruebas PISA }\end{array}$} & \multicolumn{4}{|c|}{ Tipo de establecimiento } \\
\hline & Público & Privado subvencionado & Privado independiente & Total \\
\hline \multicolumn{5}{|l|}{ Panel a } \\
\hline \multicolumn{5}{|c|}{2 horas o menos de estudio/tarea fuera de colegio } \\
\hline \multirow[t]{2}{*}{ Matemática } & 365 & 415 & 422 & 381 \\
\hline & {$[3.17]$} & {$[7.08]$} & {$[18.59]$} & {$[3.16]$} \\
\hline \multirow[t]{2}{*}{ Lectura } & 369 & 433 & 423 & 388 \\
\hline & {$[3.86]$} & {$[9.58]$} & [21.39] & {$[3.50]$} \\
\hline \multirow[t]{2}{*}{ Ciencia } & 380 & 440 & 432 & 398 \\
\hline & {$[3.99]$} & {$[6.32]$} & [21.18] & {$[3.37]$} \\
\hline \multicolumn{5}{|c|}{ Más de 2 horas de estudio/tarea fuera de colegio } \\
\hline \multirow[t]{2}{*}{ Matemática } & 401 & 445 & 442 & 421 \\
\hline & [4.89] & [ 5.79] & [12.87] & [3.68] \\
\hline \multirow[t]{2}{*}{ Lectura } & 410 & 471 & 464 & 437 \\
\hline & [6.05] & {$[6.01]$} & [10.94] & [3.97] \\
\hline \multirow[t]{2}{*}{ Ciencia } & 419 & 471 & 458 & 441 \\
\hline & {$[5.56]$} & {$[5.38]$} & [19.03] & [4.25] \\
\hline
\end{tabular}

\begin{tabular}{|c|c|c|c|c|}
\hline \multirow{2}{*}{\multicolumn{5}{|c|}{$\begin{array}{l}\text { Panel b } \\
\text { Menos de } 6 \text { horas de clases por semana }\end{array}$}} \\
\hline & & & & \\
\hline \multirow[t]{2}{*}{ Matemática } & 365 & 430 & 437 & 389 \\
\hline & {$[3.53]$} & {$[6.63]$} & {$[12.95]$} & {$[3.75]$} \\
\hline \multirow[t]{2}{*}{ Lectura } & 370 & 447 & 444 & 398 \\
\hline & {$[3.59]$} & {$[6.74]$} & {$[10.69]$} & {$[3.83]$} \\
\hline \multirow[t]{2}{*}{ Ciencia } & 377 & 445 & 446 & 402 \\
\hline & {$[3.40]$} & {$[6.69]$} & {$[11.03]$} & {$[3.62]$} \\
\hline \multicolumn{5}{|c|}{ Más de 6 horas de clases por semana } \\
\hline \multirow[t]{2}{*}{ Matemática } & 386 & 439 & 430 & 406 \\
\hline & {$[4.97]$} & {$[6.35]$} & {$[13.09]$} & {$[3.76]$} \\
\hline \multirow[t]{2}{*}{ Lectura } & 388 & 458 & 465 & 420 \\
\hline & {$[6.29]$} & {$[7.67]$} & {$[23.01]$} & {$[4.97]$} \\
\hline \multirow[t]{2}{*}{ Ciencia } & 402 & 459 & 472 & 428 \\
\hline & {$[5.50]$} & {$[8.61]$} & {$[14.49]$} & [4.64] \\
\hline
\end{tabular}

Cuando se condiciona en otras circunstancias, ya sea por nivel educativo de los padres, nivel socioeconómico cultural o clima educativo, los alumnos que asisten a escuelas privadas son aquellos que presentan en promedio un desempeño educativo más alto respecto a quienes 
asisten a las escuelas públicas. Los puntajes entre los alumnos que asisten a escuelas privadas independientes y subvencionadas no difieren significativamente, sin embargo el desvío estándar computado en el caso de los colegios privados independientes son más grandes debido a la menor cantidad de observaciones que tienen estos grupos de alumnos. La evidencia sugiere que en todos los casos los grupos de alumnos de escuelas públicas son los que obtienen menores puntajes en las pruebas respecto a los demás (ver Tabla A3.1 del Apéndice 3).

\subsection{Resultados}

En esta sección se presentan los principales resultados del trabajo. Las diferentes tablas muestran los resultados de los test de las hipótesis propuestas. En el caso donde no se rechaza la igualdad entre dos distribuciones de puntajes de alumnos se presenta el estadístico del test de Wald. Si a partir del test de Wald se rechaza la hipótesis nula de que las dos distribuciones son iguales, entonces se presentan los resultados de los test de dominancia estocástica. Solo se muestran los resultados del test de DESO si no es posible inferir DEPO (debido a que DEPO implica DESO). En los casos donde los test sugieran dominancia estocástica de una distribución sobre la otra, se muestra la relación de preferencia entre ambas distribuciones. En todos los casos se trabaja con niveles de significancia de hasta el $10 \%$.

Gráficamente, si se comparan las funciones de distribución acumulada de resultados educativos tanto de matemáticas como de lectura y ciencia entre alumnos que asisten a la escuela pública, privada subvencionada e independiente se observa que el grupo que tiene más probabilidades de presentar puntajes más altos en las pruebas PISA son los grupos de alumnos que asisten a escuelas privadas (ver Gráfico A3.1 en Apéndice 3). Lo encontrado gráficamente, es consistente con los resultados de los test estadísticos de dominancia estocástica de la Tabla 3.4. 
Tabla 3.4: Test de igualdad y de dominancia estocástica de distribuciones de puntajes de matemática según tipo de establecimiento

\begin{tabular}{|c|c|c|}
\hline & \multicolumn{2}{|c|}{ Muestra general } \\
\hline & Pública & Privada subvencionada \\
\hline \multicolumn{3}{|l|}{ Matemática } \\
\hline Pública & - & - \\
\hline Privada subvencionada & $\succ_{1}$ & - \\
\hline & {$[14587.97 ; 1.99]$} & \\
\hline Privada independiente & $\begin{array}{c}\succ_{1} \\
{[8551.93 ; 0,0]}\end{array}$ & $\begin{array}{c}=W \\
{[84.4]}\end{array}$ \\
\hline \multicolumn{3}{|l|}{ Lectura } \\
\hline Pública & - & - \\
\hline Privada subvencionada & $\succ_{1}$ & - \\
\hline & {$[16717.46 ; 0.00]$} & \\
\hline Privada independiente & $\frac{\succ_{1}}{[9152.56 ; 0.00]}$ & $\begin{array}{c}={ }_{W} \\
{[42.2]}\end{array}$ \\
\hline \multicolumn{3}{|l|}{ Ciencia } \\
\hline Pública & - & - \\
\hline Privada subvencionada & $\begin{array}{c}\succ_{1} \\
{[16487.2 ; 0.00]}\end{array}$ & - \\
\hline Privada independiente & $\begin{array}{c}\succ_{1} \\
{[8731.4 ; 0.00]}\end{array}$ & $\begin{array}{c}={ }_{W} \\
{[56.3]}\end{array}$ \\
\hline
\end{tabular}

Nota: estadístico del test entre corchetes. Para el test de dominancia, el primer estadístico corresponde al test: columna domina a la fila y el segundo estadístico corresponde al test: fila domina a la columna. $={ }_{W}$ : las distribuciones son iguales según el test de Wald; $\succ_{i}$ : la fila domina en sentido estocástico de orden $i$ a la columna; $\prec_{i}$ : la columna domina en sentido estocástico de orden $i$ a la fila.

Fuente: Elaboración propia en base a datos PISA de los años 2006, 2009 y 2012 .

Las pruebas de hipótesis indican que la distribución de puntajes de las escuelas privadas dominan en sentido estricto según los criterios de dominancia estocástica de primer y segundo orden a la de las escuelas públicas. Mostrando que los alumnos de las escuelas privadas tienen más ventajas que las escuelas públicas para cualquier puntaje $y .{ }^{10}$ Esto es, los alumnos de escuelas privadas tienen más probabilidades de obtener resultados educativos más altos que los de la escuela pública. Por lo tanto, podemos rechazar la existencia de IOP entre escuelas públicas y privadas ya que CI1bis no se cumple y la distribución de las privadas es preferida en sentido estocástico a la de las escuelas públicas. Más aún, tampoco se cumple con el CI2bis que es condición suficiente para que exista igualdad de oportunidades en sentido débil, por lo que tampoco se cumple con la definición débil IOP-D3.

En cuanto a las distribuciones de escuelas privadas subvencionadas e independientes, no se rechaza la hipótesis de igualdad de distribuciones. Ambos grupos de alumnos presentan ventajas similares, es decir, tienen las mismas probabilidades de obtener diferentes resultados educativos, sugiriendo la existencia de condiciones necesarias para que haya IOP entre ambos tipos de establecimientos. Sin embargo, solo se podrá concluir que existe IOP entre estos dos tipos de escuelas si se asume que todos los factores excluidos son independientes al tipo de establecimiento al que asisten los alumnos.

A partir de estos resultados, es interesante analizar si alumnos que se esfuerzan igual presentan las mismas probabilidades de obtener determinados puntajes independientemente

\footnotetext{
${ }^{10}$ Aquí el término ventajas se refiere a que los alumnos tienen más probabilidad de obtener resultados más altos en las pruebas.
} 
del tipo de establecimiento al cual asisten. El Gráfico A3.2 del Apéndice 3 muestra las distribuciones acumuladas de los puntajes de alumnos de distintos establecimientos que dedican similares cantidades de horas al estudio. Gráficamente, es posible observar que el grupo de alumnos de escuelas públicas que realizan un esfuerzo bajo es estrictamente dominado por los demás grupos. Es decir, es el que presenta menos ventajas respecto al resto de los grupos. Además, los resultados de la Tabla 3.5 muestran que en todos los casos, ya sea que se realice un esfuerzo alto o bajo, las distribuciones de las escuelas privadas dominan estocásticamente a las distribuciones de las escuelas públicas. Es decir, los alumnos de escuelas públicas a pesar de realizar el mismo esfuerzo corren con menores ventajas que los alumnos de escuelas privadas. Esto sugiere que tanto las condiciones necesarias como suficientes no están dadas para que exista IOP entre alumnos que asisten a escuelas públicas y escuelas privadas. Por otro lado, nuevamente a partir del test de Wald no se rechaza la igualdad entre las distribuciones de las escuelas privadas subvencionadas e independientes.

Tabla 3.5: Test de igualdad y de dominancia estocástica de distribuciones de puntajes de matemática según tipo de establecimiento y esfuerzo.

\begin{tabular}{|c|c|c|c|c|}
\hline & \multicolumn{2}{|c|}{2 hs o menos de tareas } & \multicolumn{2}{|c|}{ Más de 2 hs de tareas } \\
\hline & Pública & Privada subvencionada & Pública & Privada subvencionada \\
\hline \multicolumn{5}{|l|}{ Matemática } \\
\hline Pública & - & - & - & - \\
\hline Privada subvencionada & $\begin{array}{c}\succ_{1} \\
{[1038.76 ; 0.00]}\end{array}$ & - & $\begin{array}{c}\succ_{1} \\
{[889.22 ; 0.00]}\end{array}$ & - \\
\hline Privada independiente & $\begin{array}{c}\succ_{1} \\
{[439.41 ; 0.00]}\end{array}$ & $\begin{array}{c}={ }_{W} \\
{[12.9]}\end{array}$ & $\frac{\succ_{1}}{[398.56 ; 0.00]}$ & $\begin{array}{c}=W \\
{[8.5]}\end{array}$ \\
\hline \multicolumn{5}{|l|}{ Lectura } \\
\hline Pública & - & - & - & - \\
\hline Privada subvencionada & $\begin{array}{c}\succ_{1} \\
{[1439.17 ; 0.00]}\end{array}$ & - & $\frac{\succ_{1}}{[1291.58 ; 0.00]}$ & - \\
\hline Privada independiente & $\succ_{1}$ & $\begin{array}{c}={ }_{W} \\
{[36.2]}\end{array}$ & {$[520.91 ; 0.00]$} & $\begin{array}{l}={ }_{W} \\
{[9.0]}\end{array}$ \\
\hline \multicolumn{5}{|l|}{ Ciencia } \\
\hline Pública & - & - & - & - \\
\hline Privada subvencionada & $\begin{array}{c}\succ_{1} \\
{[1653.29 ; 0.00]}\end{array}$ & - & $\begin{array}{c}\succ_{1} \\
{[1336.12 ; 0.00]}\end{array}$ & - \\
\hline Privada independiente & $\begin{array}{c}\succ_{1} \\
{[356.82 ; 0.00]}\end{array}$ & $\begin{array}{c}=W \\
{[40.9]}\end{array}$ & $\stackrel{\succ_{1}}{[258.34 ; 0.00]}$ & $\begin{array}{c}=W \\
{[46.4]}\end{array}$ \\
\hline
\end{tabular}

\footnotetext{
Nota: estadístico del test entre corchetes. Para el test de dominancia, el primer estadístico corresponde al test: columna domina a la fila y el segundo estadístico corresponde al test: fila domina a la columna. ${ }_{W}$ : las distribuciones son iguales según el test de Wald; $\succ_{i}$ : la fila domina en sentido estocástico de orden $i$ a la columna; $\prec_{i}$ : la columna domina en sentido estocástico de orden $i$ a la fila.

Fuente: Elaboración propia en base a datos PISA de los años 2006, 2009 y 2012.
}

Cuando se condiciona por la variable cantidad de horas de clases de matemáticas, lengua y ciencia, respectivamente, y por el tipo de establecimiento educativo, se encuentra un resultado similar a los presentados en las Tablas 3.4 y 3.5. Los alumnos que asisten a la escuela pública son aquellos que presentan menos ventajas respecto de los alumnos que asisten a establecimientos privados. En todos los casos los test arrojan que las distribuciones de puntajes de las escuelas públicas son dominadas por la de las escuelas privadas. Los casos donde el test de Wald no rechaza la igualdad de distribuciones corresponden a las distribuciones entre los tipos de escuelas privadas, subvencionadas e independientes. 
Tabla 3.6: Test de igualdad y de dominancia estocástica de distribuciones de puntajes de matemática según tipo de establecimiento y factor aceptable.

\begin{tabular}{|c|c|c|c|c|}
\hline & \multicolumn{2}{|c|}{6 hs o menos de tareas } & \multicolumn{2}{|c|}{ Más de 6 hs de tareas } \\
\hline & Pública & Privada subvencionada & Pública & Privada subvencionada \\
\hline \multicolumn{5}{|l|}{ Matemática } \\
\hline Pública & - & - & - & - \\
\hline Privada subvencionada & $\begin{array}{c}\succ_{1} \\
{[5337.27 ; 6.16]}\end{array}$ & - & $\begin{array}{c}\succ_{1} \\
{[1383.61 ; 0.00]}\end{array}$ & - \\
\hline Privada independiente & {$[3282.44 ; 0.00]$} & $\begin{array}{c}={ }_{W} \\
{[55.7]}\end{array}$ & {$[433.61 ; 0.00]$} & $\begin{array}{c}={ }_{W} \\
{[17.3]}\end{array}$ \\
\hline \multicolumn{5}{|l|}{ Lectura } \\
\hline Pública & - & - & - & - \\
\hline Privada subvencionada & $\begin{array}{c}\succ_{1} \\
{[7635.36 ; 0.00]}\end{array}$ & - & $\begin{array}{c}\succ_{1} \\
{[1912.25 ; 0.00]}\end{array}$ & - \\
\hline Privada independiente & {$[3302.53 ; 0.00]$} & $\begin{array}{c}={ }_{W} \\
{[42.8]}\end{array}$ & {$\left[\begin{array}{c}\succ_{1} \\
{[785.07 ; 0.00]}\end{array}\right.$} & $\begin{array}{c}={ }_{W} \\
{[111.4]}\end{array}$ \\
\hline \multicolumn{5}{|l|}{ Ciencia } \\
\hline Pública & - & - & - & - \\
\hline Privada subvencionada & $\begin{array}{c}\succ_{1} \\
{[6087.61 ; 0.00]}\end{array}$ & - & $\begin{array}{c}\succ_{1} \\
{[1108.29 ; 0.00]}\end{array}$ & - \\
\hline Privada independiente & $\begin{array}{c}\succ_{1} \\
{[2820.04 ; 0.00]}\end{array}$ & $\begin{array}{c}={ }_{W} \\
{[28.0]}\end{array}$ & $\frac{\succ_{1}}{[757.81 ; 0.00]}$ & $\begin{array}{c}={ }_{W} \\
{[30.6]}\end{array}$ \\
\hline
\end{tabular}

\footnotetext{
Nota: estadístico del test entre corchetes. Para el test de dominancia, el primer estadístico corresponde al test: columna domina a la fila y el segundo estadístico corresponde al test: fila domina a la columna. ${ }_{W}$ : las distribuciones son iguales según el test de Wald; $\succ_{i}$ : la fila domina en sentido estocástico de orden $i$ a la columna; $\prec_{i}$ : la columna domina en sentido estocástico de orden $i$ a la fila.

Fuente: Elaboración propia en base a datos PISA de los años 2006, 2009 y 2012.
}

Ahora cabe preguntarse si los resultados anteriores entre escuelas públicas y privadas son robustos al condicionar por otras variables de circunstancias como el nivel educativo de los padres, nivel socioeconómico cultural o características del colegio. A continuación se presentan los diferentes test de las distribuciones de los puntajes de la prueba de matemática. Los resultados de lectura y de ciencia se encuentran en el apéndice del capítulo donde es posible observar que las tres pruebas conducen a conclusiones similares.

Los resultados de los test de las distribuciones de matemática de alumnos de distintos tipos de establecimientos y nivel educativo de los padres que tienen similar horas de clases se muestran en la Tabla 3.7. Se puede observar que, en general, el grupo de alumnos de escuelas privadas cuyos padres tienen un alto nivel educativo domina a los grupos de alumnos de escuelas públicas cualquiera sea el nivel educativo de los padres de estos últimos y la cantidad de horas de clases impartidas.

En el caso de los alumnos que tienen más de 6 horas de clases de matemática, el test de Wald arroja que los que asisten a colegios privados independientes con padres con educación media presentan las mismas ventajas que los alumnos que asisten las escuelas públicas cuyos padres tienen un nivel educativo ya sea bajo, medio o alto. Otro grupo de alumnos que presentan similares ventajas que los alumnos de las escuelas públicas, son los alumnos que asisten a la escuela privada subvencionada cuyos padres tienen un nivel educativo bajo.

Los resultados son distintos si se consideran los alumnos con 6 horas o menos de clases de matemática por semana, ya que las distribuciones de los alumnos que asisten a las escuelas privadas dominan a las distribuciones de los alumnos de escuelas públicas. Solamente los alumnos de escuelas públicas con padres con educación alta y media igualan en ventajas al 
grupo de alumnos de escuelas subvencionadas con padres con educación baja. Mostrando para los demás grupos de alumnos de escuelas públicas que no se cumple el CI1bis ni el CI2bis y, por lo tanto, en la mayoría de los casos no se satisfacen las condiciones de IOP entre escuelas públicas y privadas. Finalmente, los test indican que las distribuciones de alumnos que asisten a escuelas privadas, subvencionadas e independientes, con padres con educación alta y media son iguales.

En el caso de lectura y ciencia, las Tablas A3.2 y A3.3 del Apéndice 3 describen resultados similares a los de matemática. Los alumnos que tienen más de 6 horas de clases de escuelas públicas presentan iguales distribuciones que los alumnos de escuelas privadas con padres con nivel educativo bajo. En el caso de esfuerzo bajo ( 6 horas o menos de clases) solo los alumnos de escuelas pública con padres con educación alta y media igualan en ventajas a los alumnos de escuelas privadas subvencionadas con padres con nivel educativo bajo. Mostrando que el grupo que presenta menos oportunidades educativas respecto a todos los demás es el grupo de alumnos que asisten a la escuela pública, tienen menos horas de clases y cuyos padres poseen un nivel educativo bajo. 
Tabla 3.7: Test de igualdad y de dominancia estocástica de distribuciones de puntajes de matemática según tipo de establecimiento, esfuerzo y nivel educativo de los padres.

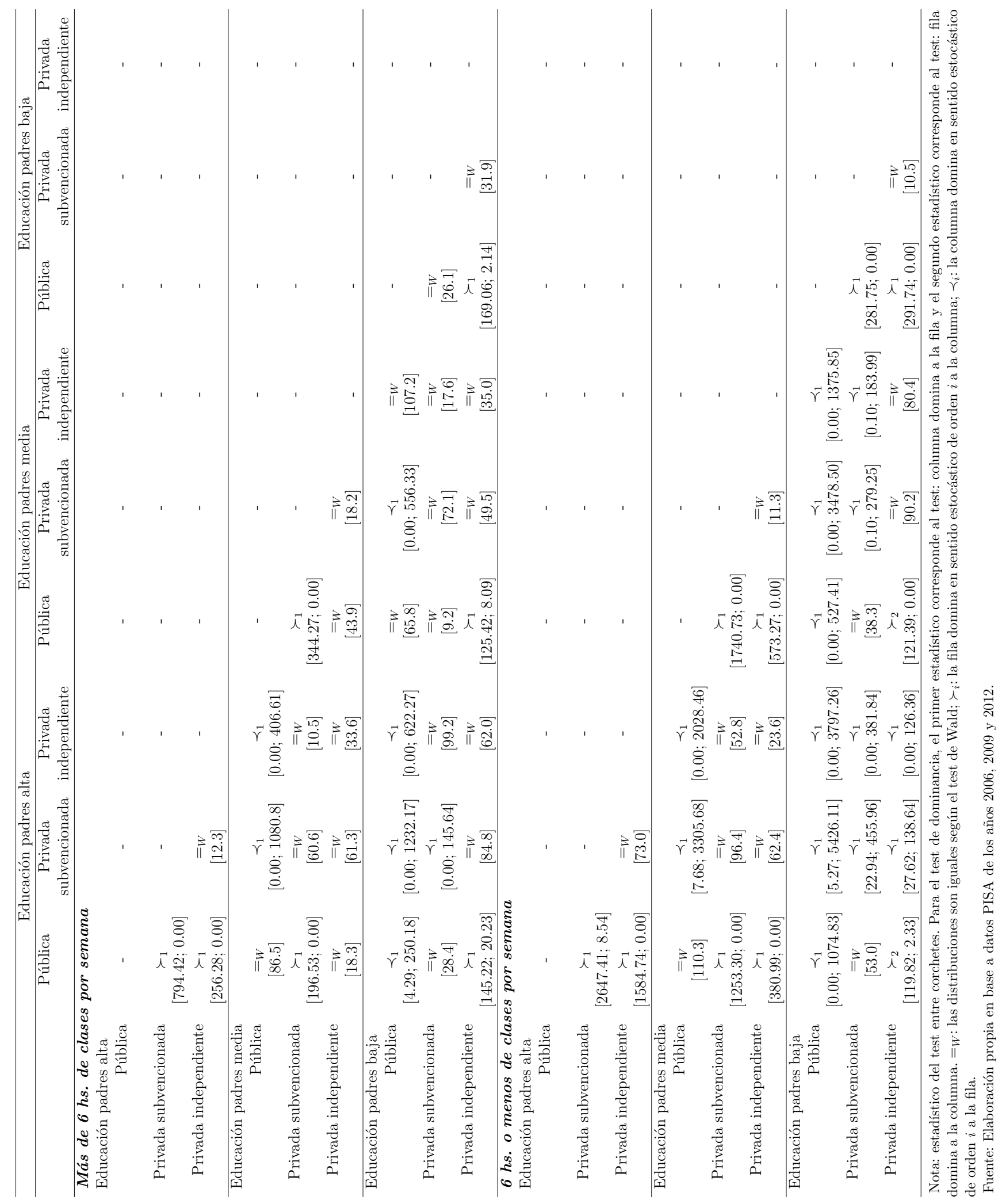


Las relaciones de preferencia según el tipo de establecimiento, esfuerzo y nivel socioeconómico cultural de la familia del alumno se resumen en la Tabla 3.8 (los resultados de lectura y ciencia se muestran en las tablas A3.4 y A3.5 del Apéndice 3). En general, puede observarse que el grupo de alumnos con más ventajas son los alumnos que asisten a la escuela privada y que tienen un contexto familiar favorable, es decir, un nivel socioeconómico cultural alto. La distribución de este grupo de alumnos domina estocásticamente las distribuciones de los alumnos de las escuelas públicas y el resto de los alumnos de las escuelas privadas (salvo los alumnos de escuelas privadas con más de 6 horas de clases cuyos padres presentan nivel educativo medio). Los resultados son similares para el caso de lectura.

Respecto a las demás relaciones, los alumnos que dedican más de 6 horas de clases a matemáticas o lectura de escuelas públicas con nivel socioeconómico alto, medio y bajo presentan iguales ventajas respecto a los alumnos de nivel socioeconómico bajo que asisten a escuelas privadas subvencionadas e independientes. Solo el grupo de alumnos de nivel socioeconómico alto iguala en ventaja al grupo de alumnos de nivel socioeconómico medio que asiste a la escuela privada. En general, puede interpretarse que para estos alumnos se cumplen las condiciones necesarias para que exista IOP entre escuelas públicas y privadas. Para el caso de 6 horas o menos, en la mayoría de los casos, la condición necesaria no se cumple, mostrando así que los alumnos de escuelas privadas presentan más ventajas respecto a los de escuela pública. Por otro lado, los resultados para ciencia muestran que los alumnos de escuelas públicas con nivel socioeconómico alto tienen ventajas similares que los alumnos de escuelas privadas pero solo en el caso de esfuerzo alto (más de 6 horas de clases). En cuanto a los grupos de alumnos que asisten a escuelas públicas, los alumnos con nivel socioeconómico alto presentan en todos los casos más ventajas respecto a los alumnos de nivel socioeconómico medio y bajo. 
Tabla 3.8: Test de igualdad y de dominancia estocástica de distribuciones de puntajes de matemática según tipo de establecimiento, esfuerzo y nivel socioeconómico familiar.

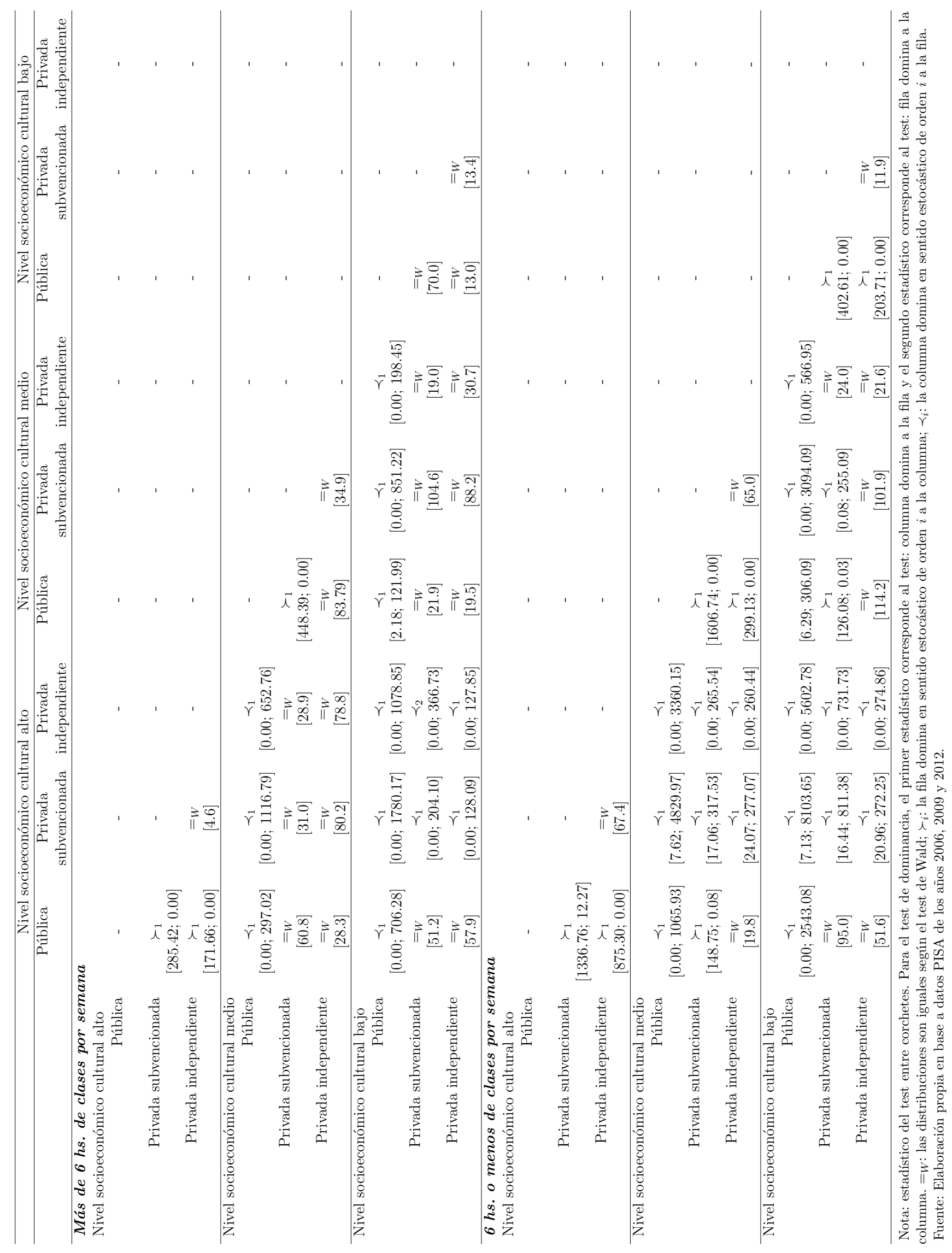


Según los resultados presentados en la primera columna de la Tabla 3.9, en el caso de más de 6 horas de clases, los alumnos de escuelas públicas con clima educativo alto igualan en ventajas a los alumnos de escuelas independientes con clima educativo alto y medio, así como a los alumnos de escuelas subvencionadas con clima educativo medio. El grupo de alumnos que asisten a escuelas subvencionadas de clima educativo alto resulta ser el grupo que presenta más ventajas respecto a los alumnos de escuelas públicas. En el caso de alumnos que van a escuelas donde tienen 6 horas o menos de clases, la distribución que domina al resto de las distribuciones no solo corresponde a la de los alumnos de escuelas privadas subvencionadas de clima educativo alto sino también a la de los alumnos de escuelas independientes de clima educativo alto. Solo los alumnos de escuelas públicas con clima educativo alto equiparan en ventajas a los alumnos de escuelas privadas con clima educativo medio y bajo. Las distribuciones de puntajes de los demás alumnos de escuelas públicas con clima educativo medio y bajo son dominadas por la de los alumnos de escuelas privadas con clima alto y medio y la de los alumnos de la pública con clima educativo alto.

Las Tablas A3.6 y A3.7 del Apéndice 3 muestran resultados distintos que los de matemática. Para lectura, solo es posible no rechazar la igualdad de distribuciones entre los alumnos con más de 6 horas de clases de escuelas públicas con clima educativo alto y alumnos de escuelas privadas (subvencionada e independientes) con clima educativo medio. A su vez solo los alumnos de escuelas públicas con clima educativo medio presentan igual distribución que los alumnos de escuelas privadas independiente con clima educativo también medio. En el caso de los grupos que tienen menos de 6 horas de clases, los alumnos de la escuela pública de clima educativo alto presentan iguales ventajas que los alumnos de escuelas privadas subvencionadas de contexto educativo medio.

Para las distribuciones de puntajes de ciencia los resultados sugieren, en general, que no existen las condiciones para IOP entre escuelas públicas y privadas. Solamente se puede no rechazar igualdad entre las distribuciones de los alumnos de escuelas públicas con clima educativo alto y de los alumnos de escuelas privadas (subvencionadas e independientes) con clima educativo alto y medio. Nuevamente las distribuciones de alumnos de escuelas públicas de contexto medio y bajo son dominadas por las distribuciones de alumnos de escuelas privadas y públicas con clima educativo similar o mayor. 
Tabla 3.9: Test de igualdad y de dominancia estocástica de distribuciones de puntajes de matemática según tipo de establecimiento, esfuerzo y clima educativo.

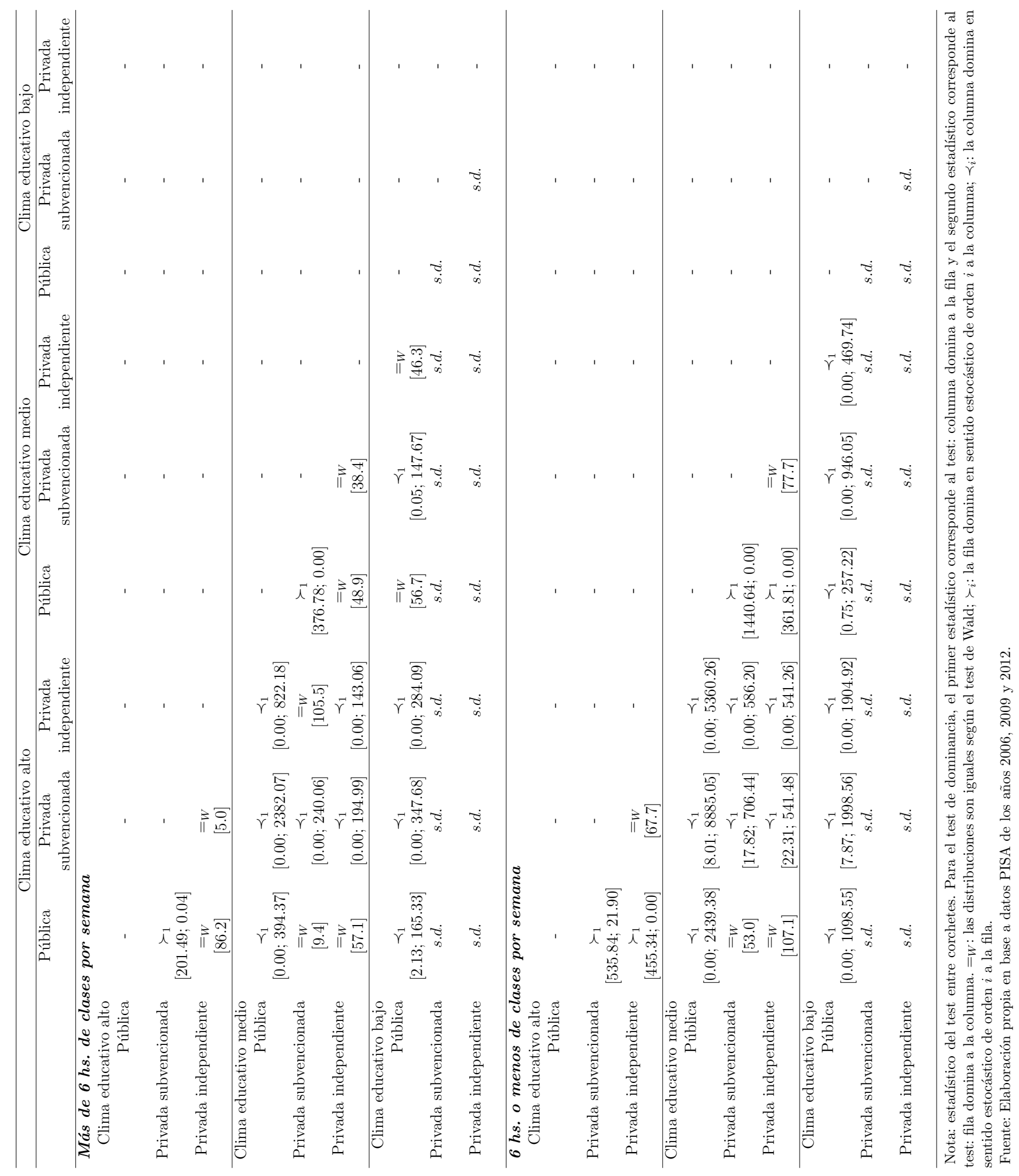


Las heterogeneidades dentro de cada tipo de establecimiento, público, privado subvencionado o independiente, pueden provenir de la localización geográfica del establecimiento así como de características en los directivos, profesores, recursos educativos que disponen las escuelas. Si bien no es posible analizar heterogeneidades por localidad ya que PISA solo ofrece información representativa a nivel país, se pueden distinguir los tipos de establecimiento según la calidad de sus recursos educativos y profesores. Incluir otras características de las escuelas puede aportar un análisis más claro respecto a las posibles heterogeneidades dentro de cada tipo de establecimiento. Las Tablas 3.10 y 3.11 complementan los resultados encontrados sobre clima educativo del colegio. Las Tablas A3.8 a A3.11 del Apéndice 3 también describen las relaciones entre las distribuciones condicionando también por recursos educativos del colegio y calidad de los profesores para el caso de lectura y ciencia.

A partir de los resultados de las tablas, se puede observar que al condicionar por la calidad de recursos educativos, para los grupos de alumnos que tienen menos de 6 horas de clases no se cumplen las condiciones necesarias y suficientes para que exista IOP entre escuelas públicas y privadas. En todos los casos los alumnos con más ventajas son aquellos que asisten a escuelas privadas. Al tener en cuenta los alumnos que tienen 6 horas o más de clases, se observa que el grupo de alumnos de escuelas públicas que equipara en ventajas a los alumnos de escuelas privadas con recursos educativos bajos o medios son aquellos cuyas escuelas tienen una alta calidad en sus recursos educativos.

En cuanto a la calidad de los profesores del colegio se observa que en el caso de alumnos con menos horas de clases no están dadas las condiciones necesarias ni suficientes para que exista IOP entre escuelas públicas y privadas. Los alumnos de escuelas privadas tienen más ventajas que los de escuela pública. Como era de esperar, el grupo de alumnos de escuelas públicas con más desventajas son aquellos cuyos colegios tienen profesores de baja calidad educativa. En el caso de alumnos que asisten a colegios que imparten más de 6 horas de clases, se encuentra nuevamente que los alumnos de escuelas públicas con calidad de profesores media y baja son los que presentan menos ventajas respecto a los demás grupos de alumnos. En cambio, no se observan diferencias entre las distribuciones de los alumnos de escuelas públicas con calidad de profesores alta y de los alumnos de escuelas privadas en general. Esto sugiere que la calidad de los profesores puede ser un factor relevante para propiciar las condiciones necesarias para igualar el campo de juego entre alumnos de escuelas públicas y privadas. 
Tabla 3.10: Test de igualdad y de dominancia estocástica de distribuciones de puntajes de matemática según tipo de establecimiento, esfuerzo y calidad de recursos educativos.

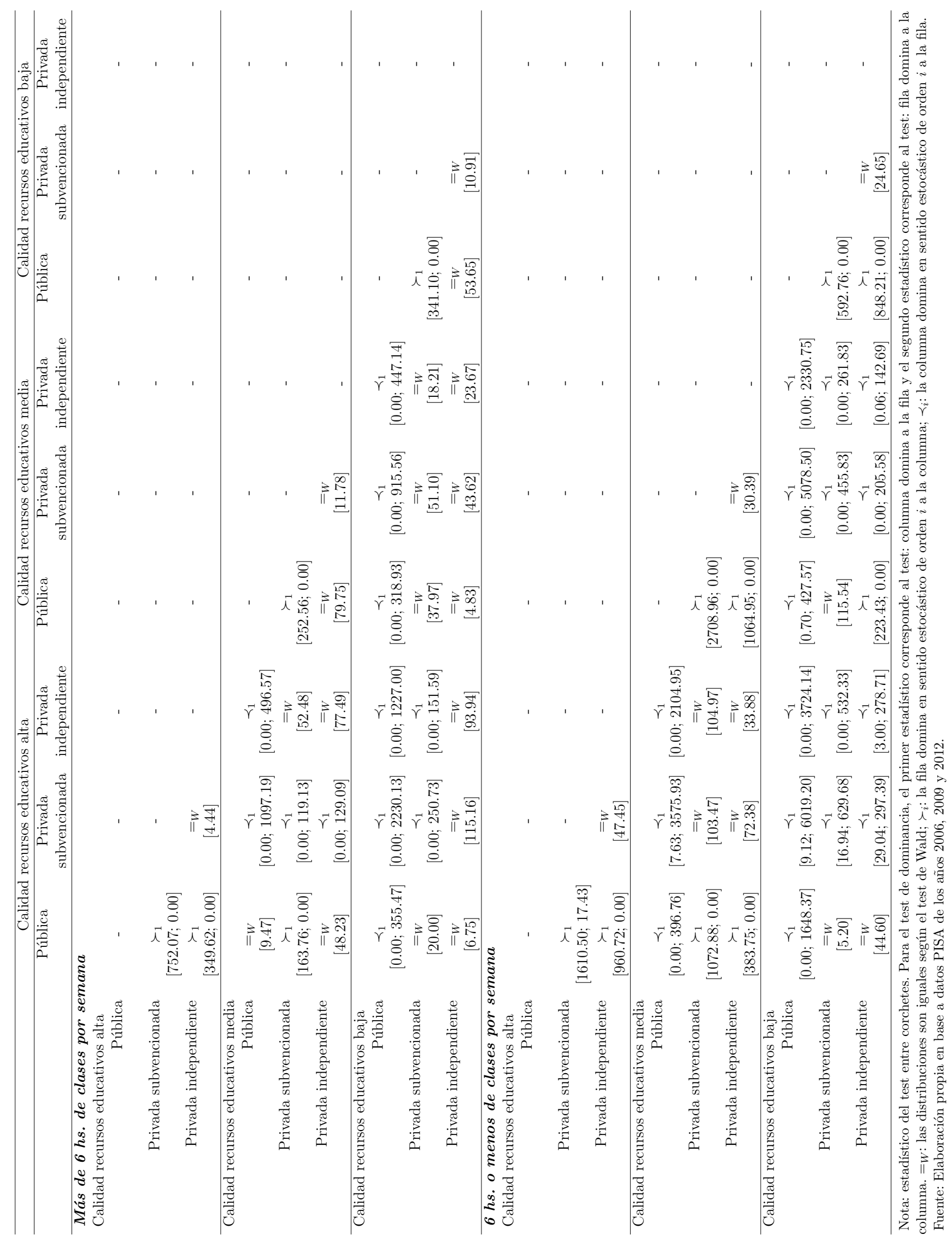


Tabla 3.11: Test de igualdad y de dominancia estocástica de distribuciones de puntajes de matemática según tipo de establecimiento, esfuerzo y calidad de los profesores del colegio.

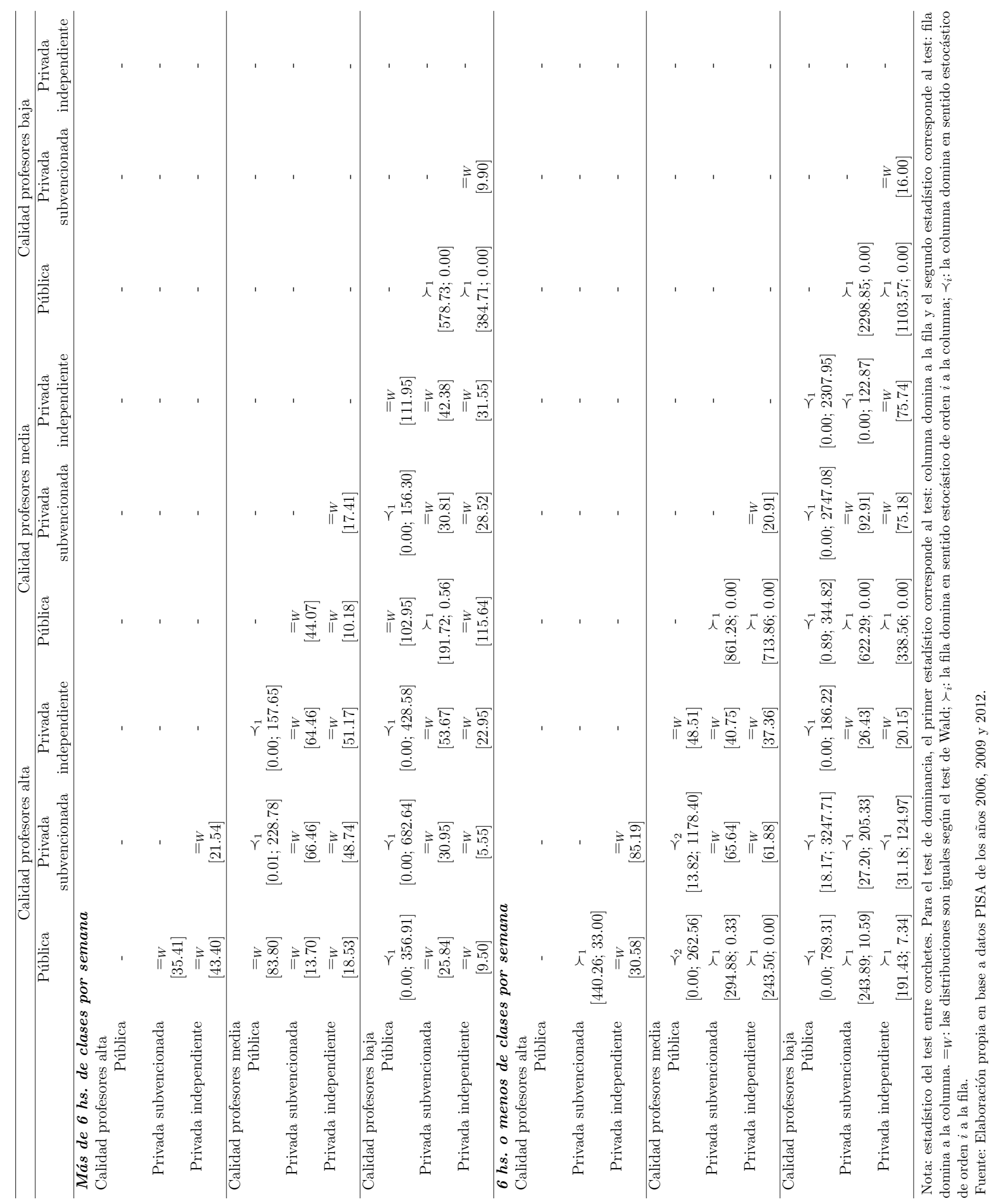


En síntesis, se observa que solo para algunos grupos de alumnos se encuentran dadas las condiciones para la existencia de IOP entre los distintos tipos de establecimientos educativos. En general, los grupos de alumnos que asisten a escuelas públicas presentan iguales oportunidades que los alumnos que asisten a escuelas privadas y provienen de contextos más desfavorables (circunstancias bajas o medias). Estos resultados ofrecen evidencia de porqué las familias se han volcado en enviar a sus hijos a escuelas privadas en los últimos años. Los alumnos de contextos más desfavorecidos que asisten al sistema privado pueden alcanzar las mismas oportunidades educativas que aquellos que asisten a las escuelas públicas que provienen de contextos más favorables (mayor nivel educativo de los padres, socioeconómico y recursos educativos del colegio).

Los demás casos muestran una clara preferencia por las distribuciones de alumnos de escuelas privadas. La distribución de este grupo de alumnos domina a la mayoría del resto de las distribuciones. Los resultados sugieren que el grupo de alumnos de escuelas privadas de contextos favorables (padres con alto nivel educativo, alto nivel socioeconómico familiar y clima educativo del colegio alto) es el grupo con más ventajas sobre los demás, más aún si se considera solo alumnos con esfuerzo bajo. Luego le siguen los alumnos de escuelas privadas independientes. Los alumnos de escuelas públicas con contextos medios o bajos son los más desfavorecidos. Si se tiene en cuenta que las pruebas PISA evalúan competencias de los alumnos, es decir, las capacidades necesarias para poder desarrollarse en una sociedad, se encuentra que los alumnos que asisten a escuelas públicas no disponen de las mismas capacidades para desarrollarse plenamente en un futuro que alumnos que asisten a escuelas privadas, en especial, si estos últimos presentan circunstancias favorables.

Un ejercicio interesante que puede arrojar un mayor entendimiento es analizar si las preferencias entre las distribuciones de resultados de los alumnos cambian si se consideran alumnos de escuelas secundarias o alumnos de escuela primaria. Se podría pensar que al comienzo de la etapa educativa todos los alumnos parten con las competencias que el hogar y su familia le han transmitido y a medida que transcurren su etapa dentro del sistema educativo, éste último complementa dichas competencias. Si la escuela pública jugara un papel igualador de oportunidades, entonces independientemente del comienzo de cada alumno debiera asegurar que los alumnos finalicen con iguales ventajas que los alumnos de escuela privada y no que dichas ventajas, a medida que avanzan en los niveles educativos, sean cada vez más distintas. Trabajando con esta posible hipótesis, se realizó un análisis para el caso de matemática a partir de la bases de datos del Tercer Estudio Regional Comparativo y Explicativo (TERCE) que realiza el Laboratorio Latinoamericano de Evaluación de la Calidad de la Educación (LLECE) de la UNESCO. Esta base de datos corresponde a un programa internacional de la UNESCO que analiza las competencias de los alumnos de las escuelas primarias (más precisamente alumnos de tercer y sexto grado) de varios países, entre ellos se encuentra Argentina. Si bien no son estrictamente comparables los resultados obtenidos con PISA, para el nivel primario se encuentra, a diferencia de los alumnos de la secundaria, que los alumnos de escuelas públicas y privadas que provienen de contextos similares cumplen con la condición necesaria para que haya IOP. Esto podría sugerir, aunque solo es una conjetura, que las oportunidades de los alumnos de escuelas públicas y privadas se vuelven más desiguales a medida que transcurren por el sistema educativo del país.

\subsection{Conclusiones}

Las oportunidades educativas ocupan un lugar central para igualar el campo de juego de las personas. Al comienzo de este capítulo se hizo la pregunta si en Argentina la escuela pública continúa teniendo un papel igualador de oportunidades. Más precisamente, si los 
alumnos que asisten a las escuelas públicas presentan las mismas probabilidades de obtener determinados desempeños educativos que los alumnos de las escuelas privadas.

Para contestar si existe evidencia de IOP entre los alumnos que asisten a distintos tipos de establecimientos, se compararon funciones de distribución de resultados educativos de diferentes grupos de alumnos que asisten a escuelas públicas y a escuelas privadas. El objetivo es probar si alumnos que se esfuerzan igual y difieren en circunstancias tienen las mismas probabilidades de obtener determinados desempeños educativos. Para ello se ordenan las distribuciones de desempeño de los diferentes grupos de alumnos utilizando principios de dominancia estocástica de primer y segundo orden. En los casos donde no es posible inferir que una distribución es preferida sobre otra y, por lo tanto, no es posible ordenarlas de acuerdo a una relación de preferencia, se deduce que están dadas las condiciones para que exista IOP entre dichos grupos de alumnos.

Los principales resultados de este capítulo sugieren que los grupos de alumnos de escuelas públicas más favorecidos por circunstancias externas no pueden competir con los alumnos de las escuelas privadas con circunstancias similares sino que solo con aquellos con circunstancias más desfavorecidas. Más aún, en general, los alumnos de la escuela pública de contextos desfavorecidos tampoco consiguen igualar oportunidades con los alumnos de escuelas privadas con similares o peores circunstancias. Esto sugiere que los alumnos de la escuela pública de contextos menos favorecidos son los que menos ventajas tienen ya que para estos alumnos no están dadas las condiciones necesarias ni suficientes para la existencia de IOP. Los resultados son robustos al condicionar por distintos esfuerzos y diferentes variables de circunstancia de los alumnos.

Cabe destacar algunas consideraciones finales sobre los resultados encontrados y el método utilizado en este capítulo. En principio, solo se provee evidencia sobre si están dadas las condiciones para que haya IOP educativas pero no se ofrece una cuantificación ni medición del grado de IOP. El método se basa en obtener una relación de preferencia entre los diferentes grupos de alumnos en cuanto a las ventajas de cada uno sobre los demás pero no qué tan diferentes son dichas ventajas. Es decir, no es posible conocer qué tan alejada se encuentra la escuela pública de su rol igualador de oportunidades.

Por otro lado, a lo largo de este capítulo se incorpora el esfuerzo individual como un factor esencial para el análisis de IOP, no solo desde el marco teórico sino también en el análisis empírico. No obstante, la tarea de encontrar una variable que refleje los esfuerzos de los alumnos no es sencilla. En primer lugar, se consideraron las horas de estudio del alumno ya que las mismas pueden constituir un factor controlable por el individuo y realizar dicha acción conlleva un costo de oportunidad que se puede asociar a un tipo de esfuerzo. En segundo lugar, se consideraron las horas de clases que tiene el alumno en la escuela, si bien estrictamente no corresponde a un tipo de esfuerzo individual ya que son las autoridades educativas de las respectivas jurisdicciones quienes establecen los días de clases así como las horas, las mismas se pueden asociar a una desigualdad socialmente aceptable.

Una ventaja que surge de la metodología es que no es necesario realizar supuestos sobre la relación entre el esfuerzo y las circunstancias observadas. Esto resulta muy conveniente en el caso de educación ya que por ejemplo el tiempo de estudio podría estar relacionado con el entorno del alumno. Aun así el análisis reposa en algunos supuestos sobre las variables no observadas y es preciso preguntarse si se está dispuesto a aceptar estos supuestos y qué tanto los factores no observables de interés para el análisis pueden considerarse independientes. En particular, se asume que tanto las circunstancias y esfuerzos no observados son independientes de las circunstancias y esfuerzos observados. Por definición, se asume que el esfuerzo es una variable controlable completamente por los individuos independiente de las circunstancias, con lo cual es poco probable que los demás esfuerzos no observados estén 
relacionados con las circunstancias ya que se estaría en contra del modelo que sustenta la teoría de IOP. En cuanto al supuesto de independencia de las circunstancias no observadas el mismo puede ser más difícil de aceptar.

A partir de lo anterior, se advierte que los resultados encontrados pueden recaer en problemas de endogeneidad e interpretaciones desacertadas sobre los efectos del tipo de escuela. Sin embargo, el principal interés no corresponde a si la escuela pública genera IOP (efecto causal) sino a si determinados grupos de alumnos de escuelas públicas que comparten características similares igualan en ventajas a los grupos de alumnos de escuela privada, independientemente de cuáles son los factores que generan las respectivas distribuciones de resultados. Con lo cual el problema de endogeneidad en los resultados no debería restringir completamente las conclusiones, pero si lleva a tener muy en cuenta el alcance de las conclusiones del estudio.

La ventaja de utilizar un método no paramétrico para estimar las distribuciones y ordenarlas siguiendo un criterio de dominancia es que es más flexible y los resultados que se obtienen son robustos para cualquier función de preferencia creciente y cóncava. Sin embargo, una de las mayores desventajas que tiene el uso de estas técnicas no paramétricas, a diferencia de sus contrapartes paramétricas, es que requieren un enorme esfuerzo computacional. Probar las condiciones de existencia de IOP implica un exigente e intensivo uso de los datos. Otra limitación del método utilizado en este estudio es el número de variables de circunstancias y esfuerzo con la que es posible trabajar. Mientras más variables se utilizan más grupos de alumnos surgen y la cantidad de observaciones en cada uno se reduce considerablemente. La contrastación empírica realizada en este trabajo puede ser perfeccionada extendiendo la metodología a otros métodos no paramétricos de estimación con mejores propiedades estadísticas (Fan y Gijbels, 1996; Schimek, 2000) y otros test como los propuestos en Bierens (1982); Fan y Li (2000), entre otros. No obstante, el uso de estos últimos métodos no es directo dado las particularidades que presenta la muestra de PISA (valores plausibles, muestreo, ponderación), por lo que resulta imperioso un profundo análisis sobre la factibilidad metodológica de estas posibles líneas de acción futura.

Por último, este capítulo aporta nueva evidencia empírica sobre IOP entre escuelas públicas y privadas en Argentina a partir de un análisis no paramétrico, permitiendo un mayor entendimiento de las desigualdades educativas existentes del país. 


\section{Comentarios finales}

En los últimos años, los estudios sobre igualdad de oportunidades han contribuido no solo en forma teórica sino empírica a la literatura de distribución. Si bien varios estudios proveen mediciones sobre desigualdad de oportunidades, son menos los que se enfocan en las oportunidades educativas. Esta tesis se centra en analizar las posibles desigualdades de oportunidades educativas que existen en Argentina brindando mediciones e indicadores sobre el grado y existencia de las mismas.

El Capítulo 1 sugiere que si bien en el país los jóvenes tienen las mismas oportunidades de acceso a la educación, no enfrentan las mismas oportunidades de acceso a una calidad educativa alta y en el desarrollo de sus competencias. De esta manera los alumnos de contextos más desfavorecidos no cuentan con las mismas ventajas al ingresar al mercado laboral o a la educación universitaria. Más aún, el Capítulo 2 muestra que la desigualdad de oportunidades del desempeño educativo de los alumnos no ha disminuido fuertemente desde el año 2000. La localización geográfica, los recursos educativos y las características de los padres son los factores que han contribuido a aumentar esta desigualdad. El Capítulo 3 sugiere que los alumnos que asisten a escuelas públicas y privadas están lejos de enfrentar las mismas oportunidades. En general, se obtiene que solo los alumnos de escuelas públicas beneficiados por circunstancias más favorables presentan las mismas oportunidades que alumnos de escuelas privadas con circunstancias más desfavorables. Los alumnos que asisten a escuelas privadas de contextos más favorables presentan las mayores ventajas respecto al resto.

Los resultados encontrados significan una contribución a la literatura en varios aspectos. Primero, se proveen mediciones sistemáticas para Argentina sobre el grado de desigualdad de oportunidades educativas de los últimos 10 años centrándose en tres dimensiones: acceso, calidad y desempeño a partir de modelos paramétricos y la estimación de distribuciones contrafactuales. Segundo, la definición de desigualdad de oportunidades implica en sí analizar las fuentes de la misma. Es decir, separar la desigualdad que proviene de las variables aceptables y la que proviene de las variables no aceptables. Para ello se utiliza un método de microdescomposición bastante flexible que permite analizar la contribución individual de cada variable más allá de la media de la distribución con el fin de analizar los cambios de la desigualdad de oportunidades independientemente de los cambios distributivos totales. Por último, también se brinda evidencia empírica sobre la hipótesis de que, en la actualidad, las escuelas públicas no tienen un papel significativo en la igualación de oportunidades.

Como se ha mencionado a lo largo de los capítulos ciertas limitaciones, algunas metodológicas y otras filosóficas, en las estimaciones y mediciones de la desigualdad de oportunidades implican una amplia agenda y desafíos metodológicos, permaneciendo varias líneas de investigación abiertas. En particular, cabe señalar que al trabajar con el desempeño educativo de los alumnos, los resultados obtenidos corresponden a nivel país, es decir, que son resultados agregados. Si bien, en el Capítulo 1 se encuentra que, en general, la desigualdad de 
oportunidades en el acceso a la educación media de las regiones va en línea con lo que sucede a nivel nacional, en lo que respecta al desempeño educativo de los alumnos, las escuelas son muy heterogéneas y dicha heterogeneidad se suele verificar entre y dentro de las provincias. Por lo tanto, los resultados agregados de desempeño obtenidos pueden no tener en cuenta algunas situaciones provinciales o locales. La disponibilidad futura de datos adecuados a nivel desagregado (como los operativos de la DINIECE, la evaluación nacional APRENDER, entre otros) haría conveniente extender el análisis a nivel provincia o localidad.

En cuanto a los posibles sesgos de estimación, es bien conocido que los estudios empíricos de educación pueden traer consigo problemas de endogeneidad. Los resultados de los tres capítulos se basan en algunos supuestos sobre las variables no observadas y se argumenta que, en algunos casos, los resultados encontrados pueden interpretarse como una desigualdad de oportunidades mínima. Sin embargo, es necesario continuar investigando estrategias que puedan ser utilizadas en los modelos de igualdad de oportunidades educativas para superar estos supuestos y evitar posibles sesgos de estimación así como estimadores inconsistentes.

Finalmente, hasta donde se conoce existe escasa evidencia sobre desigualdad de oportunidades educativas en Argentina en el marco de la teoría de igualdad de oportunidades. El principal objetivo de esta tesis es aportar evidencia empírica sobre el rol igualador del sistema educativo del país a través de un análisis riguroso del tema entendiendo, por supuesto, que existe un largo camino de investigaciones futuras para poder analizar este fenómeno en forma acabada. 


\section{Bibliografía}

Alejo, J., Gabrielli, M. F., y Sosa-Escudero, W. (2014). The distributive effects of education: An unconditional quantile regression approach. Revista de Análisis Económico, 29(1):5376.

Angrist, J. D. y Lavy, V. (1999). Using maimonides' rule to estimate the effect of class size on student achievement. Quarterly Journal of Economics, 114:533-575.

Angrist, J. D. y Lavy, V. (2002). New evidence on classroom computers and pupil learning. The Economic Journal, 112(482):735-786.

Arellano, M. y Bonhomme, S. (a publicarse en 2017). Handbook of Quantile Regression, chapter Sample Selection in Quantile Regression: A Survey.

Arnerson, R. J. (1989). Equality and equal opportunity for welfare. Philosophical Studies, 56(1):77-93.

Ashenfelter, O., Harmon, C., y Oosterbeek, H. (1999). A review of estimates of the schooling/earnings relationship, with tests for publication bias. Labour Economics, 6(4):453470.

Atkinson, A. B. y Bourguignon, F. (2015). Handbook of Income Distribution, volume 2, chapter Introduction: Income Distribution Today, pages xvii - lxiv.

Banerjee, A., Cole, S., Duflo, E., y Linden, L. (2005). Remedying education: Evidence from two randomized experiments in India. Technical report, National Bureau of Economic Research.

Beccaria, L., Maurizio, R., y Gustavo, V. (2014). Recent changes in wage inequality in Argentina. the role of labor formalization and other factors. MPRA Paper, (56701). University Library of Munich, Germany.

Betts, J. R. (1996). Is There a Link between School Inputs and Earnings? Fresh Scrutiny of an Old Literature, pages 141-191. Press.

Betts, J. R. y Roemer, J. E. (2006). Equalizing opportunity for racial and socioeconomic groups in the United States through educational finance reform. Schools and the equal opportunity problem. L. Woessmann and P. Peterson (eds). 
Bierens, H. J. (1982). Consistent model specification tests. Journal of Econometrics, 20:105134.

Björklund, A., Jäntti, M., y Roemer, J. E. (2012). Equality of Opportunity and the Distribution of Long-Run Income in Sweden. Social Choice and Welfare, 39:675-696.

Bourguignon, F., Ferreira, F. H. G., y Menéndez, M. (2007a). Inequality of opportunity in Brazil. Review of Income Wealth, 53(4):585-618.

Bourguignon, F., Ferreira, F. H. G., y Menéndez, M. (2013). Inequality of opportunity in Brazil: a corrigendum. Review of Income Wealth, 59(3):551-555.

Bourguignon, F., Ferreira, F. H. G., y Walton, M. (2007b). Equity, efficiency and inequality traps: A research agenda. Journal of Economic Inequality, 5(2):235-256.

Brunello, G. y Checchi, D. (2007). Does school tracking affect equality of opportunity? new international evidence. Economic Policy, 22(52):781-861.

Brunori, P., Ferreira, F. H. G., Lugo, M. A., y Peragine, V. (2013). Opportunity-sensitive poverty measurement. Policy Research Working Paper, (6728). The World Bank, African Region.

Card, D. (1999). Handbook of Labor Economics, volume 3A, chapter The Causal Effect of Education on Earning, pages 1801-1863. Elsevier.

Case, A. y Deaton, A. (1999). School inputs and educational outcomes in South Africa. Quarterly Journal of Economics, pages 1047-1084.

Checchi, D. y Peragine, V. (2005). Regional disparities and inequality of opportunity: The case of Italy. Discussion Papers, (1874):429-450. Institute for the Study of Labor (IZA).

Checchi, D. y Peragine, V. (2010). Inequality of opportunity in Italy. Journal of Economic Inequality, 8(4):429-450.

Checchi, D., Peragine, V., y Serlenga, L. (2008). Income inequality and opportunity inequality in Europe. Rivista di Politica Economica, 98(9):263.

Cohen, G. A. (1989). On the currency of egalitarian justice. Ethics, 99(4):906-944.

Coleman, J., E.Q., C., C.J., H., J., M., A.M., M., F.D., W., y R.L., Y. (1966). Equality of educational opportunity. Technical report, U.S. Government Printing Office, Washington, DC.

Davidson, R. y Duclos, J.-Y. (2000). Statistical inference for stochastic dominance and for the measurement of poverty and inequality. Econometrica, 68(6):1435-1464.

DiNardo, J., Fortin, N. M., y Lemieux, T. (1996). Labor market institutions and the distribution of wages, 1973-1992: A semiparametric approach. Econometrica, 64(5):1001-1044.

DINIECE (2015). Anuarios estadístico 2014. Technical report, Ministerio de Educación, Argentina.

DINIECE (2016). Indicadores educativos. Technical report, Ministerio de Educación, Argentina. 
Dworkin, R. (1981). What is equality? part 2: Equality of resources. Philosophy and Public Affairs, 10(4):283-345.

Essama-Nssah, B., Lambert, P. J., y others (2011). Influence functions for distributional statistics. Society for the study of Economic Inequality, ECINEQ Working Paper Series.

Fabris, J. y Montes-Rojas, G. (2014). Estudio de los determinantes de los cambios en la distribución del ingreso en Argentina en las últimas dos décadas. un análisis basado en la metodología de descomposiciones. mimeo. Anales Asociacion Argentina de Economía Política.

Fan, J. y Gijbels, I. (1996). Local Polynomial Modelling and Its Applications. London: Chapman Hall.

Fan, J. y Li, Q. (2000). Consistent model specification tests: Kernel-based tests versus Bierens' ICM Tests. Econometric Theory, 16:1016-1041.

Ferreira, F. H. G. y Gignoux, J. (2011). The measurement of inequality of opportunity: Theory and an application to Latin America. The Review of Income and Wealth, $57(4): 622-657$.

Ferreira, F. H. G. y Gignoux, J. (2014). The measurement of educational inequality: Achievement and opportunity. World Bank Economic Review. Primera vez publicado online: 20 de febrero de 2013.

Ferreira, F. H. G., Gignoux, J., y Aran, M. (2011). Measuring inequality of opportunity with imperfect data: the case of Turkey. Journal of Economic Inequality, 9(4):651-680. Springer.

Firpo, S., Fortin, N. M., y Lemieux, T. (2007). Decomposing wage distributions using recentered influence functions regressions. mimeo. University of British Columbia.

Firpo, S., Fortin, N. M., y Lemieux, T. (2009). Unconditional quantile regressions. Econometrica, 77(3):953-973.

Firpo, S. y Pinto, C. (2016). Identification and estimation of distributional impacts of interventions using changes in inequality measures. Journal of Applied Econometrics, 31(3):457-486.

Fleurbaey, M. (2008). Fairness, responsibility, and welfare. Oxford University Press.

Fortin, N. M., Lemieux, T., y Firpo, S. (2011). Decomposition methods in economics. Handbook of Labor Economics. Vol. 4 Parte A, editores Orley Ashenfelter and David Card.

Foster, J. E. y Shorrocks, A. F. (1988a). Poverty orderings. Econometrica, pages 173-177.

Foster, J. E. y Shorrocks, A. F. (1988b). Poverty orderings and welfare dominance. Social Choice and Welfare, 5:179-198.

Frölich, M. y Melly, B. (2013). Unconditional quantile treatment effects under endogeneity. Journal of Business Economic Statistics, 31(3):346-357. Taylor Francis Journals.

Fuchs, T. y Wößmann, L. (2004). Computers and student learning: bivariate and multivariate evidence on the availability and use of computers at home and at school. Brussels Economic Review, 47(3-4):359-386. 
Fuchs, T. y Wößmann, L. (2007). What accounts for international differences in student performance? a re-examination using PISA data. Empirical Economics, 32(2):433-464.

Gamboa, L. F. y Waltenberg, F. D. (2012). Inequality of opportunity for educational achievement in Latin America: Evidence from PISA 2006-2009. Economics of Education Review, 31(5):694-708.

Gamboa, L. F. y Waltenberg, F. D. (2015). Measuring inequality of opportunity in education by combining information on coverage and achievement in PISA. Educational Assessment, $20(4): 320-337$.

Gasparini, L., Jaume, D., M., S., y E., V. (2011). La segregación entre escuelas públicas y privadas en Argentina. reconstruyendo la evidencia. Desarrollo Económico-Revista de Ciencias Sociales, 51(202-203):189-219.

Gasparini, L. C. (2002). On the measurement of unfairness. an application to high school attendance in Argentina. Social Choice and Welfare, 19(4):795-810.

Glewwe, P., Kremer, M., y Moulin, S. (2009). Many children left behind? Textbooks and test scores in Kenya. American Economic Journal: Applied Economics, 1(1):112-35.

Hanushek, E. A. (1979). Conceptual and empirical issues in the estimation of educational production functions. Journal of human Resources, pages 351-388.

Hanushek, E. A. (1986). The economics of schooling: Production and efficiency in public schools. Journal of economic literature, 24(3):1141-1177.

Hanushek, E. A. (1997). Assessing the effects of school resources on student performance: An update. Educational evaluation and policy analysis, 19(2):141-164.

Hanushek, E. A. (2006). Chapter 14 school resources. volume 2 of Handbook of the Economics of Education, pages 865 - 908. Elsevier.

Juhn, C., Murphy, K. M., y Pierce, B. (1993). Wage inequality and the rise in returns to skill. Journal of Political Economy, 101(3):410-442.

Kanbur, R. y Stiglitz, J. E. (2016). Dynastic inequality, mobility and equality of opportunity. The Journal of Economic Inequality, pages 1-16.

Kodde, D. y Palm, F. (1986). Wald criteria for jointly testing equality and inequality restrictions. Econometrica, 54:1243-1248.

Lefranc, A., Pistolesi, N., y Trannoy, A. (2008). Inequality of opportunities vs. inequality of outcomes: Are western societies all alike? The Review of Income and Wealth, 54(4):513546.

Lefranc, A., Pistolesi, N., y Trannoy, A. (2009). Equality of opportunity and luck: Definitions and testable conditions, with an application to income in France. Journal of Public Economics, 93(11-12):1189-207.

Leuven, E., Lindahl, M., Oosterbeek, H., y Webbink, D. (2007). The effect of extra funding for disadvantaged pupils on achievement. The Review of Economics and Statistics, 89(4):721736.

Ley Nacional de Educación Nro. 26.206 (2006). Argentina. 
Machado, J. F. y Mata, J. (2005). Counterfactual decomposition of changes in wage distributions using quantile regression. Journal of Applied Econometrics, 20(4):445-465.

Melly, B. y Wuthrich, K. (a publicarse en 2017). Handbook of Quantile Regression, chapter Local quantile treatment effects.

Milanovic, B. (2015). Global inequality of opportunity: How much of our income is determined by where we live? Review of Economics and Statistics, 97(2):452-460.

OCDE (2005). Manual de análisis de datos: usuarios SPSS. PISA, OCDE, España, first edition. Traducción INECSE.

OCDE (2009). PISA Data Analysis Manual: SPSS. PISA, OECD Publishing, second edition.

Paes, B. R., Ferreira, F. H. G., Vega, J. R. M., y Chanduvi, J. S. (2008). Measuring Inequality of Opportunities in Latin America and the Caribbean. The World Bank. p. 195.

Peragine, V. (2004). Ranking income distributions according to equality of opportunity. Journal of Economic Inequality, 2(1):11-30. Springer.

Ravallion, M. (2015). Inequality when effort matters. Technical report, National Bureau of Economic Research.

Rivas, A. (2015). América Latina después de PISA: lecciones aprendidas de la educación en siete países 2000-2015. 1a ed. Fundación CIPPEC, Ciudad Autónoma de Buenos Aires.

Roemer, J. E. (1993). A pragmatic theory of responsibility for the egalitarian planner. Philosophy and Public Affairs, 22:146-166.

Roemer, J. E. (1998). Equality of Opportunity. Harvard University Pres. Cambridge.

Roemer, J. E. (2004). Generational income mobility in North America and Europe, chapter Equal opportunity and intergenerational mobility: Going beyond intergenerational income transition matrices. Cambridge University Press.

Roemer, J. E. (2012). On several approaches to equality of opportunity. Economics and Philosophy, 28:165-200. DOI:10.1017/S0266267112000156.

Roemer, J. E. y Trannoy, A. (2015). Equality of Opportunity. In Atkinson, A. B. y Bourguignon, F., editors, Handbook of Income Distribution, volume 2, chapter 4, pages 217 300. Elsevier.

Salehi-Isfahani, D., Hassine, N. B., y Assaad, R. (2014). Equality of opportunity in educational achievement in the Middle East and North Africa. The Journal of Economic Inequality, 12(4):489-515.

Schimek, M. G. (2000). Smoothing and regression: approaches, computation, and application. John Wiley \& Sons.

Schütz, G., Ursprung, H. W., y Woessmann, L. (2008). Education policy and equality of opportunity. Kyklos, 61(2):279-308. Wiley Blackwell.

SEDLAC (2014). Base de datos sección educación, CEDLAS y Banco Mundial. Accedido en abril 2014: http://sedlac.econo.unlp.edu.ar/eng/statistics.php. 
Sen, A. (2000). Handbook of Income Distribution, volume 1, chapter Social justice and the distribution of income, pages 59-85. Elsevier.

Shorrocks, A. F. (1982). Inequality decomposition by factor components. Econometrica, 50(1):193-211.

Singh (2012). Inequality of opportunity in earnings and consumption expenditure: the case of indian men. The Review of Income and Wealth, 58(1):79-106.

Vigdor, J. L., Ladd, H. F., y Martinez, E. (2014). Scaling the digital divide: home computer technology and student achievment. Economic Inquiry, 52(3):1103-1119.

Waltenberg, F. D. y Vandenberghe, V. (2007). What does it take to achieve equality of opportunity in education?: An empirical investigation based on Brazilian data. Economics of Education Review, 26(6):709-723.

Yalonetzky, G. (2012). A dissimilarity index of multidimensional inequality of opportunity. Journal of Economic Inequality, 10(3):343-373. 


\section{Anexos}

\section{Anexo: Capítulo 1}

\section{Particularidades de PISA.}

Los puntajes de las pruebas PISA de lectura, matemática y ciencias están estandarizadas de la siguiente manera:

$$
y_{i}^{P}=\widehat{\mu}+\frac{\widehat{\sigma}}{\sigma}\left(x_{i}-\mu\right)
$$

donde $y_{i}^{P}$ es el puntaje del individuo $i$ de la prueba $P$ (con $P=$ matemáticas, lectura, ciencia), $\widehat{\mu}$ es 500 y $\widehat{\sigma}$ es 100.

PISA también reporta para cada alumno 5 valores plausibles como puntaje de cada una de las pruebas $P$ :

$$
y_{i}^{P}=\left\{y_{1 i}^{P} ; \quad y_{2 i}^{P} ; \quad y_{3 i}^{P} ; \quad y_{4 i}^{P} ; \quad y_{5 i}^{P}\right\}
$$

PISA obtiene estos valores plausibles en forma aleatoria a partir de una distribución de puntajes estimada para cada estudiante. Esto se realiza para no asignar un valor puntual a la competencia del alumno, con el fin de evitar errores de medición (OCDE, 2009).

$\mathrm{Al}$ trabajar con valores plausibles, todo estadístico $\lambda$ se obtiene como ${ }^{11}$

$$
\lambda=\frac{1}{5} \sum_{v=1}^{5} \lambda_{v} .
$$

Los datos del programa presentan algunas particularidades para estimar el modelo de desempeño educativo cuya variable dependiente son los puntajes de las pruebas. Siguiendo la metodología propuesta en PISA por la OCDE (2005) para estimar los coeficientes de la ecuación (1.9) es necesario realizar, para cada una de las pruebas, una regresión para cada valor plausible por separado y luego computar un promedio de los 5 coeficientes estimados de cada variable regresora,

$$
\beta_{j}^{P}=\frac{1}{5} \sum_{v=1}^{5} \beta_{j, v}^{P}
$$

y

$$
\theta_{m}^{P}=\frac{1}{5} \sum_{v=1}^{5} \theta_{m, v}^{P}
$$

En cuanto a los errores estándar de los coeficientes de las regresiones con valores plausibles se obtienen de la siguiente manera: si $\beta$ es el estadístico poblacional y $\beta_{v}$ es el estadístico

\footnotetext{
${ }^{11}$ Los estadísticos que se obtuvieron de esta forma en este trabajo son la media, el desvío estándar, los coeficientes estimados de las regresiones de puntaje y sus errores estándares.
} 
de interés calculado sobre un valor plausible, entonces primero se calcula una varianza de imputación como:

$$
V I=\frac{1}{5-1} \sum_{v=1}^{5}\left(\beta_{v}-\beta\right)^{2}
$$

Luego, se calcula la varianza muestral $(V M)$ de los coeficientes estimados de la ecuación (1.9). Para obtener la varianza de los estimadores se combina la varianza muestral y la varianza de imputación, como se muestra a continuación

$$
V(\beta)=V M+\left(1+\frac{1}{5}\right) V I
$$

Además hay que tener en cuenta que el método de muestreo de PISA es en 2 etapas (primero se muestrean las escuelas y luego se muestrean los alumnos), y la diferencia con el muestreo aleatorio simple es que, los alumnos seleccionados del mismo establecimiento educativo no pueden considerarse como observaciones independientes apareciendo una correlación "intraclass" (intra-escuela). Esto es así porque los alumnos de un mismo establecimiento tienen entre sí más características en común que con alumnos de otras escuelas. Si no se tiene en cuenta este tipo de correlación las varianzas muestrales y los errores estándares de las estimaciones serán subestimadas, teniendo impacto sobre los resultados de las pruebas de significatividad estadística. Esto sucede porque los errores estándares que normalmente se reportan asumen que cada observación es independiente de todas las demás observaciones del conjunto de datos.

Existen posibles estrategias para solucionar este tipo de correlación en los datos. Algunas de ellas son: utilizar el método de muestreo de la encuesta según la unidad primaria de muestreo o el uso de modelos multinivel. Dado que los datos de PISA fueron recolectados a partir de una encuesta con un plan de muestreo explícito, se realiza un análisis de regresión utilizando el método de muestreo de la encuesta. Cuando se relevan las encuestas de PISA, se tiene en cuenta explícitamente la naturaleza de los datos y la correlación intra-escuela que puede surgir dentro de los establecimientos escolares. De esta manera es que para las estimaciones de la ecuación (1.9) se tiene en cuenta los procedimientos de muestreo y ponderación particulares de PISA. El método de muestreo con replicación para muestras en 2 etapas de PISA, es el método de replicación repetido equilibrado (BRR, "Balanced Repeated Replication") con la modificación de Fay con factor $k=0,5$ (OCDE, 2009).

Respecto a los modelos multinivel si bien tienen en cuenta la correlación intra-escuela y la naturaleza jerárquica de los datos (alumno-escuela), los mismos suponen efectos aleatorios por escuela. Sin embargo las escuelas se seleccionan según probabilidades proporcionales a su tamaño. Por tanto, escuelas más grandes, tienen una probabilidad mayor de selección que las escuelas más pequeñas (OCDE, 2009). Con lo cual estimar un modelo multinivel puede no ser conveniente para el análisis y en su lugar se opta por estimar un modelo de efectos fijos.

También cabe señalar que la forma de estandarización de los puntajes hace que el índice de desigualdad más adecuado de analizar en la sección 1.4.3 del capítulo sea la varianza o el desvío estándar (Ferreira y Gignoux, 2014) ya que

$$
\operatorname{Var}\left(y^{P}\right)=\operatorname{Var}\left(\widehat{\mu}+\frac{\widehat{\sigma}}{\sigma}\left(x^{P}-\mu\right)\right)=\left(\frac{\widehat{\sigma}}{\sigma}\right)^{2} \operatorname{Var}\left(x^{P}\right) .
$$


Esta medida estadística satisface los axiomas de simetría, continuidad y el principio de transferencia. La desigualdad de oportunidades absoluta de cada prueba se mide a partir de la distribución contrafáctica $\hat{y}^{P}$ a través de la varianza $\left(\operatorname{Var}\left(\hat{y}^{P}\right)\right)$, y la relativa por:

$$
\hat{\theta}_{D O}^{P}=\frac{\operatorname{Var}\left(\hat{y}^{P}\right)}{\operatorname{Var}\left(y^{P}\right)}=\frac{\operatorname{Var}\left(\hat{x}^{P}\right)}{\operatorname{Var}\left(x^{P}\right)} .
$$

A partir de las fórmulas anteriores se observa que $\hat{\theta}_{D O}^{P}$ es invariante a la estandarización luego de la transformación en cociente. Al aplicar la raíz cuadrada a dicha varianza se puede realizar el análisis a partir del desvío estándar como medida de dispersión. Entonces para cada prueba $P$ tenemos que:

$$
\hat{\theta}_{D O}=\frac{S D(\hat{y})}{S D(y)}=\sqrt{\frac{\operatorname{Var}(\hat{y})}{\operatorname{Var}(y)}}=\frac{\sqrt{\operatorname{Var}\left(\bar{X}^{\prime} \hat{\theta}+C^{\prime} \hat{\beta}\right)}}{\sqrt{\operatorname{Var}(y)}} .
$$




\section{Anexo: Capítulo 2}

\section{Detalles de estimación de regresiones de RIF para distintos $v$.}

\section{Cuantiles no condicionados}

La descomposición por cuantiles no condicionados es más flexible que la de otras medidas resumen como la varianza o el Gini, ya que en este caso los cambios distributivos se analizan en diferentes partes de la distribución de resultados. Esto permite observar cómo distintos factores tienen un impacto diferente en distintos cuantiles de la distribución. ${ }^{12}$

El cuantil $\tau$ de una distribución $F, v(F)=q_{\tau}$ se define como:

$$
Q(F, \tau)=\inf \{y \mid F(y) \geq \tau\}=q_{\tau} .
$$

La función de influencia del cuantil $\tau$ es $I F\left(y, q_{\tau}\right)=\frac{\tau-\mathbf{1}\left\{y \leq q_{\tau}\right\}}{f_{Y}\left(q_{Y}\right)}$ donde $\mathbf{1}\{\}$ es una función indicadora, $f_{Y}()$ es la función de densidad de la distribución marginal de $Y$. La función de influencia recentrada es la suma del cuantil $\tau$ y la función de influencia.

El cuantil $\tau$ de la distribución de cada grupo y de la distribución contrafactual se estima de la siguiente manera,

$$
\hat{q}_{\tau_{g}}=\operatorname{argmin}_{q} \sum_{i=1}^{N} \hat{\omega}_{g}\left(G_{i}\right)\left|y_{i}-q\right|
$$

para $g=0,1$ y

$$
\hat{q}_{\tau_{C}}=\operatorname{argmin}_{q} \sum_{i=1}^{N} \hat{\omega}_{C}\left(G_{i}\right)\left|y_{i}-q\right| .
$$

Para la descomposición se estima la regresión de RIF en función de las variables $X$, reemplazando la variable dependiente $Y$ por los valores estimados de RIF según cada grupo:

$$
\begin{aligned}
R \hat{I} F\left(y, q_{\tau_{g}}\right) & =\hat{q}_{\tau_{g}}+\hat{I F}\left(y, q_{\tau_{g}}\right) \\
& =\hat{q}_{\tau_{g}}+\frac{\tau-1\left\{y \leq \hat{q}_{\tau_{g}}\right\}}{\hat{f}_{Y_{g}}\left(\hat{q}_{\tau}\right)}
\end{aligned}
$$

$\operatorname{para} g=0,1 \mathrm{y}$

$$
\begin{aligned}
R \hat{I} F\left(y, q_{\tau_{C}}\right) & =\hat{q}_{\tau_{C}}+\hat{I F}\left(y, q_{\tau_{C}}\right) \\
& =\hat{q}_{\tau_{C}}+\frac{\tau-\mathbf{1}\left\{y \leq \hat{q}_{\tau_{C}}\right\}}{\hat{f}_{Y_{C}}\left(\hat{q}_{\tau}\right)}
\end{aligned}
$$

donde $\hat{f}_{Y_{g}}\left(\hat{q}_{\tau}\right)$ y $\hat{f}_{Y_{C}}\left(\hat{q}_{\tau}\right)$ se estiman a través del método de kernel de Epanechnikov con un

\footnotetext{
${ }^{12}$ Tener en cuenta que a diferencia de la media cuyo efecto parcial no condicionado medio $E(\partial E[Y \mid X] / \partial x)$ por ley de esperanza iteradas es igual al efecto parcial condicionado medio $\partial E[Y \mid X] / \partial x$; en el caso de los cuantiles esto no es así. Los cuantiles condicionados no se corresponden a sus contrapartes no condicionados (Firpo et al., 2009). Entonces mientras que las regresiones por MCO se pueden utilizar para estimar el efecto de $X$ ya sea en la media condicionada o en la media no condicionada. Las estimaciones de la regresión por cuantiles condicionados no se pueden usar para evaluar el efecto de un cambio de $X$ en el cuantil correspondiente de la distribución marginal de $Y$; y es necesario utilizar las estimaciones de las regresiones por cuantiles no condicionados.
} 
ancho de banda (bandwidth) de 5 .

En el caso de los cuantiles no condicionados, la regresión de RIF puede ser estimada por diferentes métodos. Al estar estrechamente vinculados a un modelo de probabilidad se puede estimar a través de un modelo lineal por MCO, un modelo Logit por Máxima Verosimilitud o una versión de Logit no paramétrica. Los tres métodos brindan estimadores válidos dado que los efectos marginales de un modelo lineal de probabilidad (RIF-MCO) o un Logit (RIFLogit o RIF-paramétrico) suelen ser similares. A los fines de comparación se estiman las regresiones de RIF por medio de MCO. Se obtienen así los estimadores de los coeficientes $\hat{\gamma}^{q_{\tau 0}}, \hat{\gamma}^{q_{\tau 1}}$ y $\hat{\gamma}^{q_{\tau C}}$.

\section{Varianza}

En el caso de la varianza, se puede estimar como

$$
\hat{\sigma}_{g}^{2}=\frac{1}{N} \sum_{i=1}^{N} \hat{\omega}_{g}\left(G_{i}\right)\left(y_{i}-\mu\right)^{2}
$$

para $g=0,1$; en el caso de la distribución contrafactual,

$$
\hat{\sigma}_{C}^{2}=\frac{1}{N} \sum_{i=1}^{N} \hat{\omega}_{C}\left(G_{i}, X_{i}\right)\left(y_{i}-\mu\right)^{2} .
$$

La función de influencia de la varianza es $\operatorname{IF}\left(y, \sigma^{2}\right)=\left(y-\int z d F_{Y}(z)\right)^{2}-\sigma^{2}$ y la función de influencia recentrada es $\operatorname{RIF}\left(y, \sigma^{2}\right)=\left(y-\int z d F_{Y}(z)\right)^{2}=(y-\mu)^{2}$ que se puede estimar a partir de la media de la distribución

$$
R \hat{I} F\left(y, \sigma^{2}\right)=(y-\hat{\mu})^{2} .
$$

Reemplazando la variable dependiente $Y$ por los valores estimados de RIF de acuerdo a cada grupo y el contrafactual, se realizan las regresiones de RIF por MCO y se obtienen los estimadores de los coeficientes $\hat{\gamma}^{\sigma_{0}^{2}}, \hat{\gamma}^{\sigma_{1}^{2}}$ y $\hat{\gamma}^{\sigma_{C}^{2}}$. A partir de estos últimos y los ponderadores es posible descomponer la brecha de las varianzas según la contribución individual de cada variable.

\section{Coeficiente de Gini}

De manera similar se puede descomponer el coeficiente de Gini. Este índice se puede expresar a partir de la curva Generalizada de Lorenz (GL) de la siguiente manera:

$$
v^{G}=1-2 \mu^{-1} H\left(F_{Y}\right)
$$

donde $H\left(F_{Y}\right)=\int_{0}^{1} G L\left(p(y), F_{Y}\right) d p(y)$ con $p(y)=F_{Y}(y)$. La función de influencia es

$$
I F\left(y ; v^{G}\right)=A\left(F_{Y}\right)+B\left(F_{Y}\right) y+C\left(y ; F_{Y}\right)
$$

donde

$$
\begin{aligned}
A\left(F_{Y}\right) & =2 \mu^{-1} H\left(F_{Y}\right) \\
B\left(F_{Y}\right) & =2 \mu^{-2} H\left(F_{Y}\right) \\
C\left(y ; F_{Y}\right) & =-2 \mu^{-1}\left(y(1-p(y))+G L\left(p(y) ; F_{Y}\right) .\right.
\end{aligned}
$$


De esta manera la RIF resulta ser

$$
R I F\left(y ; v^{G}\right)=1+B\left(F_{Y}\right) y+C\left(y ; F_{Y}\right)
$$

Reemplazando la variable dependiente $Y$ por los valores estimados de RIF de acuerdo a cada grupo y el contrafactual, se realizan las regresiones de RIF por MCO y se obtienen los estimadores de los coeficientes $\hat{\gamma}^{G_{0}}, \hat{\gamma}^{G_{1}}$ y $\hat{\gamma}^{G_{C}}$. A partir de estos últimos y los ponderadores es posible descomponer el cambio del índice de Gini según la contribución individual de cada variable. 


\section{Apéndices}

\section{Apéndice 1: Capítulo 1}

\section{Estadísticas descriptivas.}

Tabla A1.1: Puntajes de las pruebas PISA de escuelas privadas y escuelas públicas (2006, 2009, 2012).

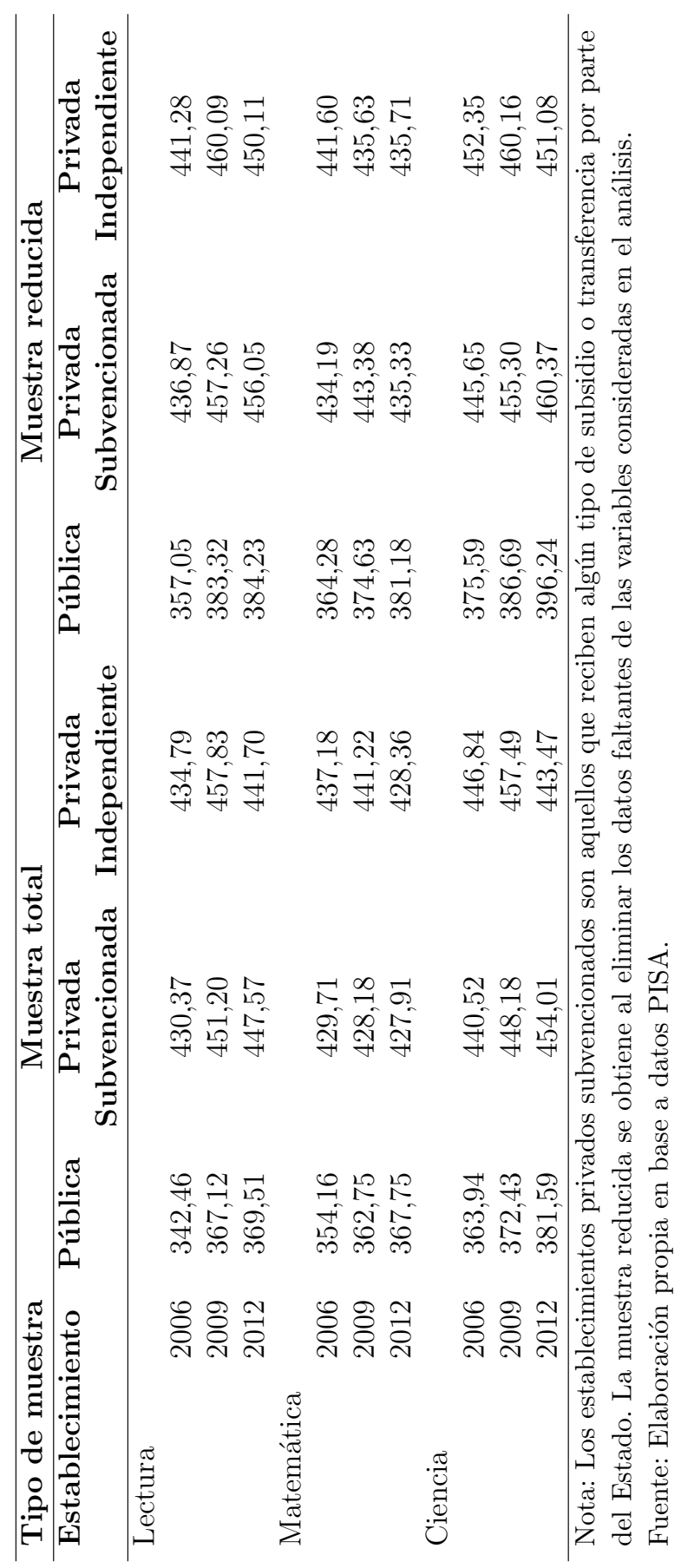


Tabla A1.2: Estadísticas descriptivas de la muestra obtenida a partir de las EPH (20032012).

\begin{tabular}{|c|c|c|c|c|c|c|c|c|c|c|}
\hline \multirow[t]{2}{*}{ Variables } & \multicolumn{2}{|c|}{ II sem-2003 } & \multicolumn{2}{|c|}{ II sem-2004 } & \multicolumn{2}{|c|}{ II sem-2005 } & \multicolumn{2}{|c|}{ II sem-2006 } & \multicolumn{2}{|c|}{ II sem-2007 } \\
\hline & Media & SD & Media & SD & Media & SD & Media & SD & Media & SD \\
\hline \multicolumn{11}{|l|}{ Resultado } \\
\hline asiste educación & 0,88 & 0,32 & 0,88 & 0,32 & 0,91 & 0,29 & 0,90 & 0,30 & 0,88 & 0,33 \\
\hline asiste educación privada & 0,29 & 0,46 & 0,30 & 0,46 & 0,31 & 0,46 & 0,32 & 0,47 & 0,27 & 0,44 \\
\hline \multicolumn{11}{|l|}{ No aceptables } \\
\hline hombre & 0,49 & 0,50 & 0,50 & 0,50 & 0,51 & 0,50 & 0,52 & 0,50 & 0,51 & 0,50 \\
\hline educpadres & 9,52 & 4,05 & 9,54 & 3,94 & 9,68 & 3,95 & 9,72 & 3,99 & 9,92 & 3,89 \\
\hline ambospadres & 0,79 & 0,41 & 0,78 & 0,41 & 0,77 & 0,42 & 0,78 & 0,41 & 0,79 & 0,41 \\
\hline ocupjefe1 & 0,10 & 0,30 & 0,10 & 0,30 & 0,12 & 0,33 & 0,12 & 0,33 & 0,13 & 0,33 \\
\hline ocupjefe2 & 0,02 & 0,15 & 0,02 & 0,14 & 0,02 & 0,14 & 0,02 & 0,13 & 0,01 & 0,11 \\
\hline ocupjefe3 & 0,24 & 0,43 & 0,28 & 0,45 & 0,29 & 0,45 & 0,29 & 0,45 & 0,29 & 0,46 \\
\hline ocupjefe4 & 0,17 & 0,37 & 0,17 & 0,37 & 0,19 & 0,39 & 0,19 & 0,39 & 0,17 & 0,37 \\
\hline ocupjefe 5 & 0,07 & 0,25 & 0,06 & 0,24 & 0,07 & 0,25 & 0,06 & 0,24 & 0,08 & 0,27 \\
\hline ocupjefe6 & 0,25 & 0,43 & 0,25 & 0,43 & 0,21 & 0,40 & 0,21 & 0,41 & 0,19 & 0,39 \\
\hline migra & 0,09 & 0,29 & 0,10 & 0,30 & 0,10 & 0,30 & 0,09 & 0,29 & 0,11 & 0,31 \\
\hline gba & 0,52 & 0,50 & 0,53 & 0,50 & 0,54 & 0,50 & 0,51 & 0,50 & 0,50 & 0,50 \\
\hline nea & 0,05 & 0,23 & 0,06 & 0,23 & 0,06 & 0,23 & 0,06 & 0,24 & 0,07 & 0,25 \\
\hline noa & 0,10 & 0,31 & 0,10 & 0,30 & 0,09 & 0,29 & 0,10 & 0,30 & 0,11 & 0,31 \\
\hline cuyo & 0,07 & 0,25 & 0,07 & 0,25 & 0,06 & 0,24 & 0,06 & 0,25 & 0,07 & 0,25 \\
\hline pampa & 0,23 & 0,42 & 0,22 & 0,41 & 0,21 & 0,41 & 0,23 & 0,42 & 0,21 & 0,41 \\
\hline \multicolumn{11}{|l|}{ Aceptables } \\
\hline pata & 0,03 & 0,17 & 0,03 & 0,16 & 0,03 & 0,17 & 0,04 & 0,20 & 0,04 & 0,19 \\
\hline edad & 15,14 & 1,36 & 15,12 & 1,34 & 15,17 & 1,34 & 15,20 & 1,34 & 15,21 & 1,34 \\
\hline grado & 0,55 & 0,50 & 0,54 & 0,50 & 0,72 & 0,45 & 0,72 & 0,45 & 0,63 & 0,48 \\
\hline Observaciones & \multicolumn{2}{|c|}{5.098} & \multicolumn{2}{|c|}{5.074} & \multicolumn{2}{|c|}{5.008} & \multicolumn{2}{|c|}{7.020} & \multicolumn{2}{|c|}{4.397} \\
\hline \multirow[t]{2}{*}{ Variables } & \multicolumn{2}{|c|}{ II sem-2008 } & \multicolumn{2}{|c|}{ II sem-2009 } & \multicolumn{2}{|c|}{ II sem-2010 } & II sem & 2011 & II sem & 2012 \\
\hline & Media & SD & Media & SD & Media & SD & Media & SD & Media & SD \\
\hline Resultado & & & & & & & & & & \\
\hline asiste educa & 0,88 & 0,32 & 0,90 & 0,29 & 0,91 & 0,28 & 0,91 & 0,29 & 0,91 & 0,28 \\
\hline asiste educación 1 & 0,30 & 0,46 & 0,30 & 0,46 & 0,32 & 0,47 & 0,30 & 0,46 & 0,30 & 0,46 \\
\hline No aceptables & & & & & & & & & & \\
\hline hombre & 0,51 & 0,50 & 0,50 & 0,50 & 0,48 & 0,50 & 0,50 & 0,50 & 0,52 & 0,50 \\
\hline educpadres & 9,88 & 3,99 & 10,05 & 4,01 & 10,08 & 3,88 & 10,14 & 3,79 & 10,30 & 3,78 \\
\hline ambospadres & 0,78 & 0,41 & 0,75 & 0,43 & 0,76 & 0,43 & 0,77 & 0,42 & 0,76 & 0,43 \\
\hline ocupjefe1 & 0,12 & 0,33 & 0,11 & 0,31 & 0,14 & 0,35 & 0,03 & 0,17 & 0,02 & 0,13 \\
\hline ocupjefe2 & 0,02 & 0,13 & 0,02 & 0,14 & 0,02 & 0,14 & 0,06 & 0,23 & 0,07 & 0,25 \\
\hline ocupjefe3 & 0,28 & 0,45 & 0,27 & 0,44 & 0,26 & 0,44 & 0,22 & 0,41 & 0,20 & 0.40 \\
\hline ocupjefe4 & 0,17 & 0,37 & 0,18 & 0,38 & 0,19 & 0,39 & 0,02 & 0,13 & 0,01 & 0,11 \\
\hline ocupjefe5 & 0,07 & 0,25 & 0,06 & 0,25 & 0,06 & 0,25 & 0,03 & 0,16 & 0,04 & 0,19 \\
\hline ocupjefe6 & 0,20 & 0,40 & 0,22 & 0,41 & 0,19 & 0,40 & 0,20 & 0,40 & 0,20 & 0,40 \\
\hline migra & 0,09 & 0,29 & 0,09 & 0,29 & 0,11 & 0,31 & 0,09 & 0,29 & 0,10 & 0,30 \\
\hline gba & 0,51 & 0,50 & 0,50 & 0,50 & 0,54 & 0,50 & 0,54 & 0,50 & 0,54 & 0,50 \\
\hline nea & 0,06 & 0,23 & 0,06 & 0,24 & 0,06 & 0,24 & 0,06 & 0,24 & 0,06 & 0,24 \\
\hline noa & 0,11 & 0,32 & 0,11 & 0,31 & 0,10 & 0,29 & 0,10 & 0,30 & 0,10 & 0,30 \\
\hline cuyo & 0,06 & 0,24 & 0,06 & 0,24 & 0,06 & 0,23 & 0,05 & 0,23 & 0,06 & 0,23 \\
\hline pampa & 0,22 & 0,41 & 0,23 & 0,42 & 0,21 & 0,40 & 0,20 & 0,40 & 0,20 & 0,40 \\
\hline Acep & & & & & & & & & & \\
\hline pata & 0,04 & 0,19 & 0,04 & 0,20 & 0,04 & 0,20 & 0,04 & 0,20 & 0,04 & 0,19 \\
\hline edad & 15,19 & 1,35 & 15,14 & 1,36 & 15,10 & 1,35 & 15,08 & 1,39 & 15,10 & 1,37 \\
\hline grado & 0,67 & 0,47 & 0,66 & 0,47 & 0,63 & 0,48 & 0,58 & 0,49 & 0,55 & 0,50 \\
\hline Observaciones & 6.7 & & 6.4 & & 4.2 & & 4.2 & & 4.0 & \\
\hline
\end{tabular}

Fuente: Elaboración propia en base a datos EPH. 
Tabla A1.3: Estadísticas descriptivas de la sub-muestra de jóvenes de 15 años obtenida a partir de las EPH (2003-2012).

\begin{tabular}{|c|c|c|c|c|c|c|c|c|c|c|}
\hline \multirow[t]{2}{*}{ Variables } & \multicolumn{2}{|c|}{ II sem-2003 } & \multicolumn{2}{|c|}{ II sem-2004 } & \multicolumn{2}{|c|}{ II sem-2005 } & \multicolumn{2}{|c|}{ II sem-2006 } & \multicolumn{2}{|c|}{ II sem-2007 } \\
\hline & Media & SD & Media & SD & Media & SD & Media & SD & Media & SD \\
\hline Resultado & & & & & & & & & & \\
\hline asiste educación 1 & 0,88 & 0,33 & 0,87 & 0,33 & 0,91 & 0,29 & 0,90 & 0,31 & 0,87 & 0,33 \\
\hline asiste educación privada & 0,25 & 0,44 & 0,29 & 0,46 & 0,29 & 0,46 & 0,32 & 0,46 & 0,29 & 0,45 \\
\hline \multicolumn{11}{|l|}{ No aceptables } \\
\hline hombre & 0,49 & 0,50 & 0,49 & 0,50 & 0,50 & 0,50 & 0,52 & 0,50 & 0,52 & 0,50 \\
\hline educpadres & 9,27 & 4,00 & 9,26 & 3,96 & 9,50 & 3,96 & 9,57 & 3,96 & 10,03 & 3,88 \\
\hline ambospadres & 0,77 & 0,42 & 0,77 & 0,42 & 0,76 & 0,43 & 0,77 & 0,42 & 0,76 & 0,43 \\
\hline ocupjefe1 & 0,10 & 0,30 & 0,10 & 0,30 & 0,13 & 0,33 & 0,13 & 0,34 & 0,12 & 0,33 \\
\hline ocupjefe2 & 0,03 & 0,16 & 0,02 & 0,14 & 0,02 & 0,15 & 0,02 & 0,14 & 0,01 & 0,10 \\
\hline ocupjefe3 & 0,22 & 0,41 & 0,27 & 0,45 & 0,29 & 0,45 & 0,28 & 0,45 & 0,27 & 0,45 \\
\hline ocupjefe4 & 0,18 & 0,39 & 0,17 & 0,37 & 0,19 & 0,39 & 0,18 & 0,39 & 0,18 & 0,38 \\
\hline ocupjefe5 & 0,06 & 0,24 & 0,06 & 0,24 & 0,07 & 0,25 & 0,06 & 0,23 & 0,08 & 0,27 \\
\hline ocupjefe6 & 0,26 & 0,44 & 0,24 & 0,43 & 0,19 & 0,40 & 0,20 & 0,40 & 0,19 & 0,39 \\
\hline migra & 0,09 & 0,29 & 0,11 & 0,31 & 0,11 & 0,32 & 0,10 & 0,30 & 0,11 & 0,31 \\
\hline gba & 0,52 & 0,50 & 0,55 & 0,50 & 0,54 & 0,50 & 0,51 & 0,50 & 0,52 & 0,50 \\
\hline nea & 0,06 & 0,23 & 0,06 & 0,23 & 0,05 & 0,22 & 0,06 & 0,24 & 0,07 & 0,26 \\
\hline noa & 0,10 & 0,31 & 0,10 & 0,30 & 0,09 & 0,29 & 0,10 & 0,30 & 0,11 & 0,31 \\
\hline cuyo & 0,07 & 0,25 & 0,06 & 0,23 & 0,06 & 0,24 & 0,06 & 0,24 & 0,06 & 0,24 \\
\hline pampa & 0,23 & 0,42 & 0,21 & 0,41 & 0,22 & 0,41 & 0,23 & 0,42 & 0,20 & 0,40 \\
\hline pata & 0,03 & 0,17 & 0,02 & 0,16 & 0,03 & 0,17 & 0,04 & 0,20 & 0,04 & 0,19 \\
\hline \multicolumn{11}{|l|}{ Aceptables } \\
\hline edad & 15,47 & 0,50 & 15,49 & 0,50 & 15,50 & 0,50 & 15,51 & 0,50 & 15,50 & 0,50 \\
\hline grado & 0,63 & 0,48 & 0,63 & 0,48 & 0,67 & 0,47 & 0,65 & 0,48 & 0,59 & 0,49 \\
\hline Observaciones & \multicolumn{2}{|c|}{2.200} & \multicolumn{2}{|c|}{2.186} & \multicolumn{2}{|c|}{2.210} & \multicolumn{2}{|c|}{3.250} & \multicolumn{2}{|c|}{1.993} \\
\hline \multirow[t]{2}{*}{ Variables } & \multicolumn{2}{|c|}{ II sem-2008 } & \multicolumn{2}{|c|}{ II sem-2009 } & \multicolumn{2}{|c|}{ II sem-2010 } & \multicolumn{2}{|c|}{ II sem-2011 } & II sem & 2012 \\
\hline & Media & $\mathrm{SD}$ & Media & SD & Media & $\mathrm{SD}$ & Media & SD & Media & SD \\
\hline Resultado & & & & & & & & & & \\
\hline asiste educa & 0,88 & 0,33 & 0,90 & 0,30 & 0,91 & 0,29 & 0,91 & 0,29 & 0,90 & 0,30 \\
\hline asiste educación privada & 0,29 & 0,45 & 0,29 & 0,45 & 0,31 & 0,46 & 0,29 & 0,45 & 0,27 & 0,45 \\
\hline No aceptables & & & & & & & & & & \\
\hline hombre & 0,51 & 0,50 & 0,51 & 0,50 & 0,50 & 0,50 & 0,50 & 0,50 & 0,50 & 0,50 \\
\hline educpadres & 9,78 & 3,94 & 9,96 & 4,00 & 9,96 & 4,07 & 10,09 & 3,68 & 10,05 & 3,73 \\
\hline ambospadres & 0,78 & 0,41 & 0,75 & 0,43 & 0,77 & 0,42 & 0,75 & 0,43 & 0,75 & 0,43 \\
\hline ocupjefe1 & 0,12 & 0,32 & 0,11 & 0,32 & 0,13 & 0,34 & 0,03 & 0,18 & 0,02 & 0,14 \\
\hline ocupjefe2 & 0,02 & 0,13 & 0,02 & 0,13 & 0,02 & 0,14 & 0,05 & 0,22 & 0,05 & 0,21 \\
\hline ocupjefe 3 & 0,26 & 0,44 & 0,29 & 0,45 & 0,25 & 0,43 & 0,21 & 0,41 & 0,20 & 0,40 \\
\hline ocupjefe 4 & 0,18 & 0,38 & 0,17 & 0,37 & 0,19 & 0,39 & 0,01 & 0,11 & 0,02 & 0,12 \\
\hline ocupjefe 5 & 0,07 & 0,25 & 0,06 & 0,24 & 0,07 & 0,26 & 0,02 & 0,14 & 0,03 & 0,18 \\
\hline ocupjefe6 & 0,21 & 0,41 & 0,20 & 0,40 & 0,19 & 0,40 & 0,20 & 0,40 & 0,19 & 0,39 \\
\hline migra & 0,09 & 0,28 & 0,09 & 0,29 & 0,10 & 0,30 & 0,08 & 0,27 & 0,12 & 0,32 \\
\hline gba & 0,51 & 0,50 & 0,51 & 0,50 & 0,54 & 0,50 & 0,53 & 0,50 & 0,54 & 0,50 \\
\hline nea & 0,06 & 0,23 & 0,07 & 0,25 & 0,07 & 0,25 & 0,07 & 0,25 & 0,07 & 0,25 \\
\hline noa & 0,11 & 0,32 & 0,10 & 0,30 & 0,09 & 0,29 & 0,11 & 0,31 & 0,10 & 0,29 \\
\hline cuyo & 0,06 & 0,24 & 0,06 & 0,24 & 0,06 & 0,23 & 0,06 & 0,23 & 0,05 & 0,23 \\
\hline pampa & 0,22 & 0,41 & 0,22 & 0,42 & 0,20 & 0,40 & 0,19 & 0,39 & 0,20 & 0,40 \\
\hline Acepta & & & & & & & & & & \\
\hline pata & 0,04 & 0,19 & 0,04 & 0,20 & 0,04 & 0,20 & 0,04 & 0,20 & 0,04 & 0,19 \\
\hline edad & 15,49 & 0,50 & 15,49 & 0,50 & 15,49 & 0,50 & 15,48 & 0,50 & 15,51 & 0,50 \\
\hline grado & 0,62 & 0,48 & 0,64 & 0,48 & 0,60 & 0,49 & 0,54 & 0,50 & 0,52 & 0,50 \\
\hline Observaciones & 3.0 & & 2.7 & & 1.8 & & 1.8 & & 1.7 & \\
\hline
\end{tabular}

Fuente: Elaboración propia en base a datos EPH. 
Tabla A1.4: Estadísticas descriptivas de la muestra obtenida a partir de PISA (2006, 2009 y 2012).

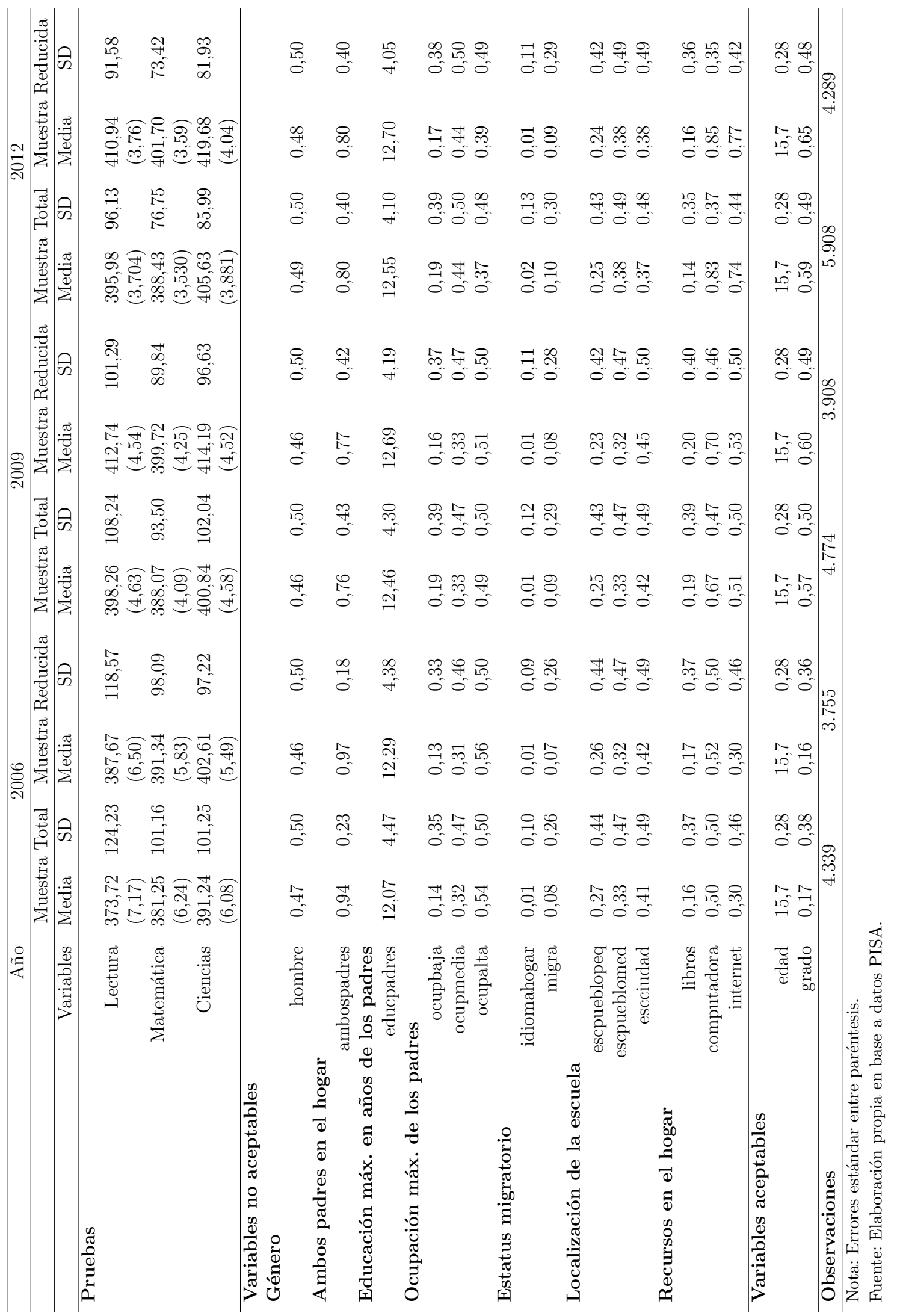




\section{Estimaciones de los modelos educativos.}

Tabla A1.5: Estimaciones del modelo de probabilidad de acceso a educación media de jóvenes entre 12 y 18 años (2003-2012).

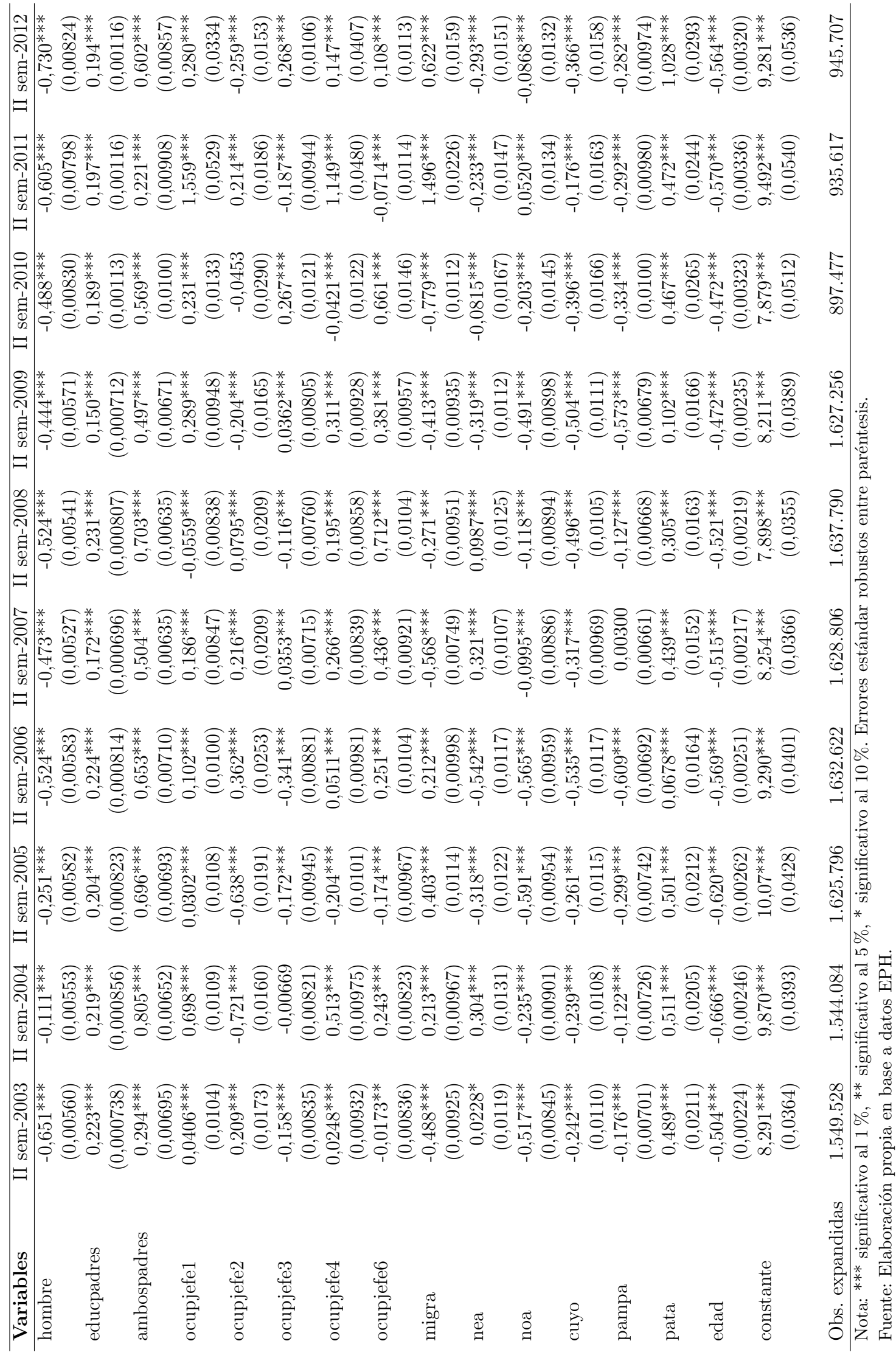


Tabla A1.6: Estimaciones del modelo de probabilidad de acceso a educación media privada de jóvenes entre 12 y 18 años (2003-2012).

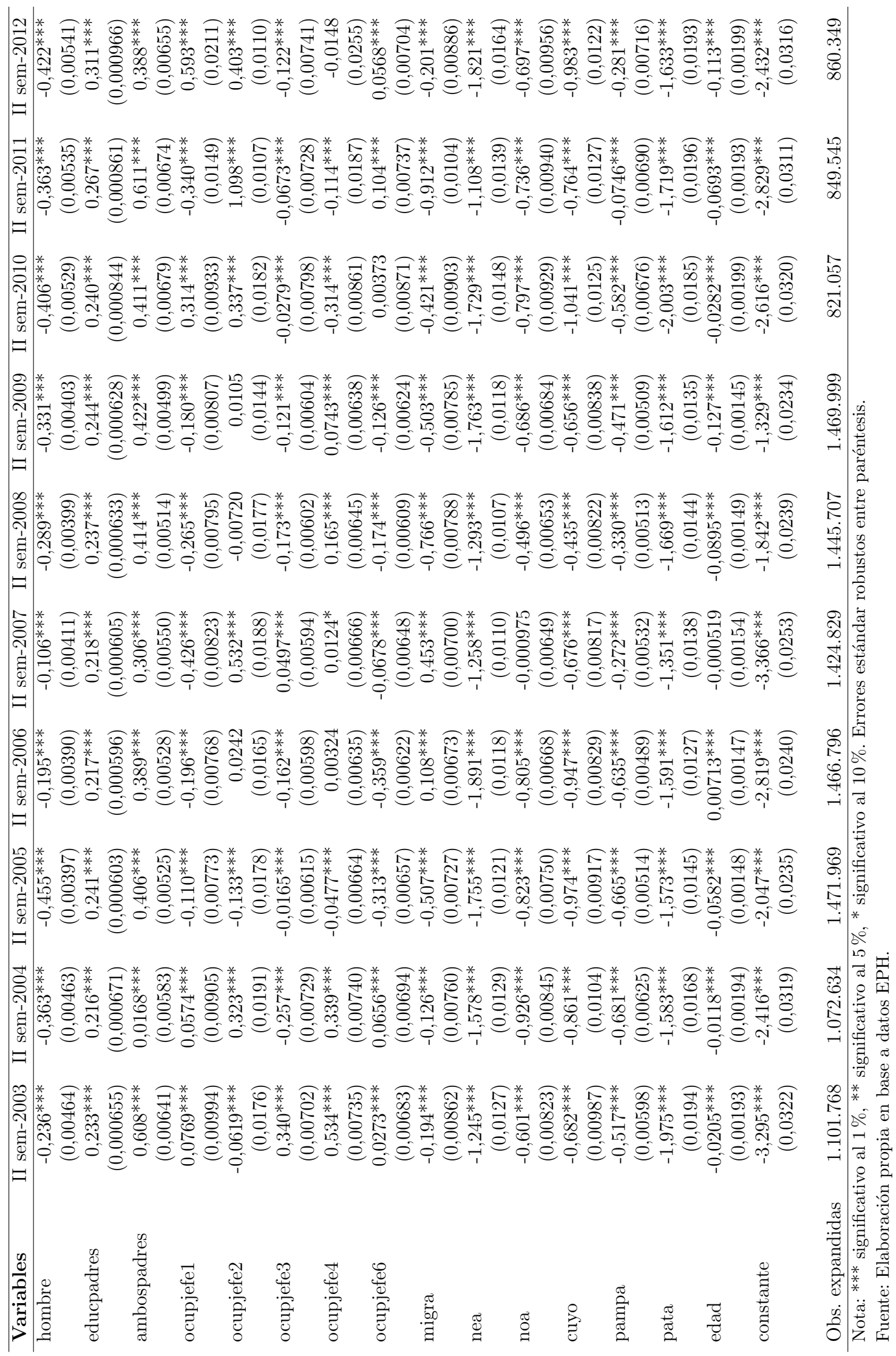


Tabla A1.7: Estimaciones del modelo de probabilidad de acceso a escuelas con puntajes promedios igual o superior al promedio de la OCDE (2006, 2009, 2012).

\begin{tabular}{|c|c|c|c|c|c|c|c|c|c|}
\hline \multirow[t]{2}{*}{ Variables } & \multicolumn{3}{|c|}{ Al menos en 1 prueba. } & \multicolumn{3}{|c|}{ Al menos en 2 pruebas. } & \multicolumn{3}{|c|}{ En las 3 pruebas. } \\
\hline & 2006 & 2009 & 2012 & 2006 & 2009 & 2012 & 2006 & 2009 & 2012 \\
\hline \multirow[t]{2}{*}{ hombre } & $-0.733^{* *}$ & $-0.293^{*}$ & -0.211 & $-0.733^{* *}$ & -0.200 & -0.129 & -0.246 & -0.106 & 0.196 \\
\hline & $(0.286)$ & $(0.163)$ & $(0.176)$ & $(0.286)$ & $(0.185)$ & $(0.189)$ & $(0.338)$ & $(0.278)$ & $(0.360)$ \\
\hline \multirow[t]{2}{*}{ ambospadres } & 0.687 & 0.336 & 0.234 & 0.687 & 0.331 & 0.0891 & 0.601 & $0.606^{*}$ & $-0.549^{*}$ \\
\hline & $(0.751)$ & $(0.204)$ & $(0.160)$ & $(0.751)$ & $(0.217)$ & $(0.207)$ & $(1.164)$ & $(0.344)$ & $(0.298)$ \\
\hline \multirow[t]{2}{*}{ educpadres } & $0.105^{* * *}$ & $0.102^{* *}$ & $0.152^{* * *}$ & $0.105^{* * *}$ & $0.132^{* *}$ & $0.228^{* * *}$ & $0.140^{* *}$ & $0.306^{* * *}$ & $0.331^{* *}$ \\
\hline & $(0.0367)$ & $(0.0393)$ & $(0.0305)$ & $(0.0367)$ & $(0.0572)$ & $(0.0414)$ & $(0.0622)$ & $(0.101)$ & $(0.132)$ \\
\hline \multirow[t]{2}{*}{ ocupbaja } & $-2.350^{* *}$ & $-0.666^{*}$ & $-0.731^{* * *}$ & $-2.350^{* *}$ & $-0.702^{* *}$ & $-0.926^{* * *}$ & & -0.254 & -0.403 \\
\hline & $(1.021)$ & $(0.337)$ & $(0.221)$ & $(1.021)$ & $(0.339)$ & $(0.288)$ & & $(0.568)$ & $(0.831)$ \\
\hline \multirow[t]{2}{*}{ ocupmedia } & $-0.666^{* *}$ & $-0.626 * * *$ & $-0.946^{* * *}$ & $-0.666^{* *}$ & $-0.663^{* *}$ & $-0.959 * * *$ & $-0.592^{*}$ & $-2.040^{* * *}$ & $-1.279^{* *}$ \\
\hline & $(0.254)$ & $(0.181)$ & $(0.194)$ & $(0.254)$ & $(0.255)$ & $(0.318)$ & $(0.333)$ & $(0.554)$ & $(0.596)$ \\
\hline \multirow[t]{2}{*}{ idiomahogar } & -0.926 & -0.0882 & -0.292 & -0.926 & 0.233 & 0.305 & & 0.672 & \\
\hline & $(2.019)$ & $(0.557)$ & $(0.678)$ & $(2.019)$ & $(0.491)$ & $(0.810)$ & & $(0.703)$ & \\
\hline \multirow[t]{2}{*}{ migra } & 0.286 & -0.0848 & -0.0629 & 0.286 & -0.106 & 0.236 & 0.107 & 0.110 & 0.695 \\
\hline & $(0.370)$ & $(0.231)$ & $(0.217)$ & $(0.370)$ & $(0.253)$ & $(0.311)$ & $(0.658)$ & $(0.283)$ & $(0.652)$ \\
\hline \multirow[t]{2}{*}{ escpueblopeq } & & -1.665 & -1.087 & & & & & & \\
\hline & & $(1.166)$ & $(1.256)$ & & & & & & \\
\hline \multirow[t]{2}{*}{ escpueblomed } & -0.0753 & -0.480 & -0.595 & -0.0753 & -0.577 & 0.826 & 0.434 & & \\
\hline & $(1.042)$ & $(0.548)$ & $(0.622)$ & $(1.042)$ & $(0.676)$ & $(0.728)$ & $(1.358)$ & & \\
\hline \multirow[t]{2}{*}{ libros } & $0.775^{* * *}$ & $0.651^{* * *}$ & $0.466^{* *}$ & $0.775^{* * *}$ & $0.559^{* *}$ & $0.591^{*}$ & $0.898^{* * *}$ & $0.813^{* * *}$ & $1.151^{* * *}$ \\
\hline & $(0.220)$ & $(0.194)$ & $(0.201)$ & $(0.220)$ & $(0.240)$ & $(0.342)$ & $(0.222)$ & $(0.285)$ & $(0.158)$ \\
\hline \multirow[t]{2}{*}{ computadora } & $1.023^{* * *}$ & $0.775^{* *}$ & 0.100 & $1.023^{* * *}$ & 0.588 & 0.410 & $0.833^{* *}$ & & 0.717 \\
\hline & (0.198) & $(0.310)$ & $(0.420)$ & $(0.198)$ & $(0.371)$ & $(0.291)$ & $(0.334)$ & & $(0.730)$ \\
\hline \multirow[t]{2}{*}{ internet } & $1.195^{* * *}$ & $1.756^{* * *}$ & $1.939 * * *$ & $1.195^{* * *}$ & $1.261^{* * *}$ & $1.640^{* *}$ & $1.234^{* * *}$ & $1.802^{*}$ & 0.832 \\
\hline & $(0.336)$ & $(0.365)$ & $(0.460)$ & $(0.336)$ & $(0.431)$ & $(0.666)$ & $(0.348)$ & (1.034) & $(0.589)$ \\
\hline \multirow[t]{2}{*}{ edad } & $-0.848^{*}$ & 0.0751 & -0.0208 & $-0.848^{*}$ & 0.0908 & -0.286 & -1.017 & 0.346 & 0.216 \\
\hline & $(0.459)$ & $(0.222)$ & $(0.211)$ & $(0.459)$ & $(0.245)$ & $(0.372)$ & $(0.863)$ & $(0.259)$ & $(0.630)$ \\
\hline \multirow[t]{2}{*}{ constante } & 7.525 & -5.999 & -5.266 & 7.525 & -6.577 & -4.244 & 8.592 & $-14.05^{* *}$ & $-14.34^{* *}$ \\
\hline & $(6.562)$ & $(3.690)$ & $(3.483)$ & $(6.562)$ & (4.197) & $(5.253)$ & $(12.59)$ & $(6.112)$ & $(7.037)$ \\
\hline Observaciones & 2,752 & 3,908 & 4,152 & 2,752 & 3,066 & 3,390 & 2,497 & 1,373 & 2,107 \\
\hline Prueba F (p-valor) & 0.000 & 0.000 & 0.000 & 0.000 & 0.000 & 0.000 & 0.000 & 0.000 & 0.000 \\
\hline
\end{tabular}


Tabla A1.8: Estimaciones del modelo de probabilidad de acceso a escuelas con puntajes promedios igual o superior al promedio de la OCDE según las distintas pruebas (2006, 2009, 2012).

\begin{tabular}{|c|c|c|c|c|c|c|c|c|c|}
\hline \multirow[t]{2}{*}{ Variables } & \multicolumn{3}{|c|}{ En lectura } & \multicolumn{3}{|c|}{ En matemática } & \multicolumn{3}{|c|}{ En ciencia } \\
\hline & 2006 & 2009 & 2012 & 2006 & 2009 & 2012 & 2006 & 2009 & 2012 \\
\hline \multirow[t]{2}{*}{ hombre } & $-0.729^{* *}$ & $-0.358^{* *}$ & -0.229 & -0.331 & 0.0172 & -0.225 & $-0.733^{* *}$ & -0.199 & 0.0134 \\
\hline & $(0.329)$ & $(0.171)$ & $(0.188)$ & $(0.286)$ & $(0.208)$ & $(0.259)$ & $(0.286)$ & $(0.192)$ & $(0.159)$ \\
\hline \multirow[t]{2}{*}{ ambospadres } & 1.351 & $0.383^{*}$ & $0.272^{*}$ & 0.135 & 0.373 & 0.0972 & 0.687 & 0.346 & -0.0939 \\
\hline & $(1.113)$ & $(0.211)$ & $(0.160)$ & $(0.696)$ & $(0.305)$ & $(0.279)$ & $(0.751)$ & $(0.226)$ & $(0.215)$ \\
\hline \multirow[t]{2}{*}{ educpadres } & $0.124^{* * *}$ & $0.136^{* * *}$ & $0.152^{* * *}$ & $0.110^{* *}$ & 0.110 & $0.238^{* * *}$ & $0.105^{* * *}$ & $0.136^{* *}$ & $0.191^{* * *}$ \\
\hline & $(0.0374)$ & $(0.0385)$ & $(0.0328)$ & $(0.0521)$ & $(0.0811)$ & $(0.0380)$ & $(0.0367)$ & $(0.0597)$ & $(0.0425)$ \\
\hline \multirow[t]{2}{*}{ ocupbaja } & $-2.134^{* *}$ & -0.612 & $-0.686^{* * *}$ & & -0.537 & $-1.102^{* * *}$ & $-2.350 * *$ & $-0.675^{*}$ & $-0.836^{* * *}$ \\
\hline & $(0.985)$ & $(0.392)$ & $(0.228)$ & & $(0.333)$ & $(0.403)$ & (1.021) & $(0.352)$ & $(0.289)$ \\
\hline \multirow[t]{2}{*}{ ocupmedia } & $-0.764^{* *}$ & $-0.728^{* * *}$ & $-0.967 * * *$ & $-0.474^{*}$ & $-1.006^{* * *}$ & $-1.663^{* * *}$ & $-0.666^{* *}$ & $-0.638^{* *}$ & $-0.608^{* * *}$ \\
\hline & $(0.299)$ & $(0.208)$ & $(0.210)$ & $(0.264)$ & $(0.376)$ & $(0.415)$ & $(0.254)$ & $(0.261)$ & $(0.183)$ \\
\hline \multirow[t]{2}{*}{ idiomahogar } & & 0.0487 & -0.179 & 0.306 & 0.407 & 0.269 & -0.926 & 0.198 & -0.403 \\
\hline & & $(0.566)$ & $(0.677)$ & $(1.741)$ & $(0.658)$ & $(0.995)$ & $(2.019)$ & $(0.510)$ & $(0.970)$ \\
\hline \multirow[t]{2}{*}{ migra } & $0.548^{*}$ & -0.0923 & -0.0475 & -0.299 & 0.0875 & 0.179 & 0.286 & -0.148 & 0.229 \\
\hline & $(0.282)$ & $(0.253)$ & $(0.205)$ & $(0.739)$ & $(0.195)$ & $(0.495)$ & $(0.370)$ & $(0.263)$ & $(0.365)$ \\
\hline \multirow[t]{2}{*}{ escpueblopeq } & & -1.577 & -0.879 & & & & & & \\
\hline & & (1.183) & $(1.264)$ & & & & & & \\
\hline \multirow[t]{2}{*}{ escpueblomed } & -0.631 & -0.698 & -0.384 & 0.995 & -1.637 & -0.493 & -0.0753 & -0.500 & 0.188 \\
\hline & $(1.277)$ & $(0.584)$ & $(0.633)$ & $(1.155)$ & $(1.270)$ & (1.188) & $(1.042)$ & $(0.679)$ & $(0.953)$ \\
\hline \multirow[t]{2}{*}{ libros } & $0.693^{* * *}$ & $0.645^{* * *}$ & $0.462^{* *}$ & $0.994^{* * *}$ & $0.790^{* * *}$ & $0.601^{*}$ & $0.775^{* * *}$ & $0.528^{* *}$ & 0.553 \\
\hline & $(0.240)$ & $(0.202)$ & $(0.213)$ & $(0.186)$ & $(0.219)$ & $(0.354)$ & $(0.220)$ & $(0.247)$ & $(0.345)$ \\
\hline \multirow[t]{2}{*}{ computadora } & $1.061^{* * *}$ & $0.993^{* * *}$ & -0.00241 & $0.852^{* * *}$ & 0.545 & 0.445 & $1.023^{* * *}$ & 0.629 & $0.684^{*}$ \\
\hline & $(0.229)$ & $(0.365)$ & $(0.414)$ & $(0.271)$ & $(0.520)$ & $(0.418)$ & $(0.198)$ & $(0.390)$ & $(0.397)$ \\
\hline \multirow[t]{2}{*}{ internet } & $1.173^{* * *}$ & $1.749^{* * *}$ & $1.887^{* * *}$ & $1.255^{* * *}$ & $1.635^{* * *}$ & $2.040^{* *}$ & $1.195^{* * *}$ & $1.247^{* * *}$ & $1.611^{* *}$ \\
\hline & $(0.382)$ & $(0.428)$ & $(0.471)$ & $(0.269)$ & $(0.434)$ & $(0.856)$ & $(0.336)$ & $(0.444)$ & $(0.661)$ \\
\hline \multirow[t]{2}{*}{ edad } & -0.825 & 0.177 & -0.0893 & -1.047 & 0.116 & 0.334 & $-0.848^{*}$ & 0.0781 & -0.229 \\
\hline & $(0.529)$ & $(0.239)$ & $(0.214)$ & $(0.694)$ & $(0.235)$ & $(0.297)$ & $(0.459)$ & $(0.251)$ & $(0.358)$ \\
\hline \multirow[t]{2}{*}{ constante } & 6.241 & $-8.394^{* *}$ & -4.274 & 9.922 & -7.427 & $-14.67^{* * *}$ & 7.525 & -6.549 & -4.427 \\
\hline & $(7.673)$ & $(3.911)$ & $(3.494)$ & $(9.940)$ & $(4.658)$ & $(4.309)$ & $(6.562)$ & $(4.304)$ & $(4.902)$ \\
\hline Observaciones & 2,735 & 3,908 & 4,152 & 2,512 & 3,066 & 3,390 & 2,752 & 3,066 & 3,390 \\
\hline Prueba F (p-valor) & 0.000 & 0.000 & 0.000 & 0.000 & 0.000 & 0.000 & 0.000 & 0.000 & 0.000 \\
\hline
\end{tabular}

Nota: ${ }^{* *}$ significativo al $1 \%,{ }^{* *}$ significativo al $5 \%,{ }^{*}$ significativo al $10 \%$. Errores estándar robustos entre paréntesis.

Fuente: Elaboración propia en base a datos PISA. 
Tabla A1.9: Estimaciones del modelo de desempeño educativo por MCO (2006, 2009 y 2012).

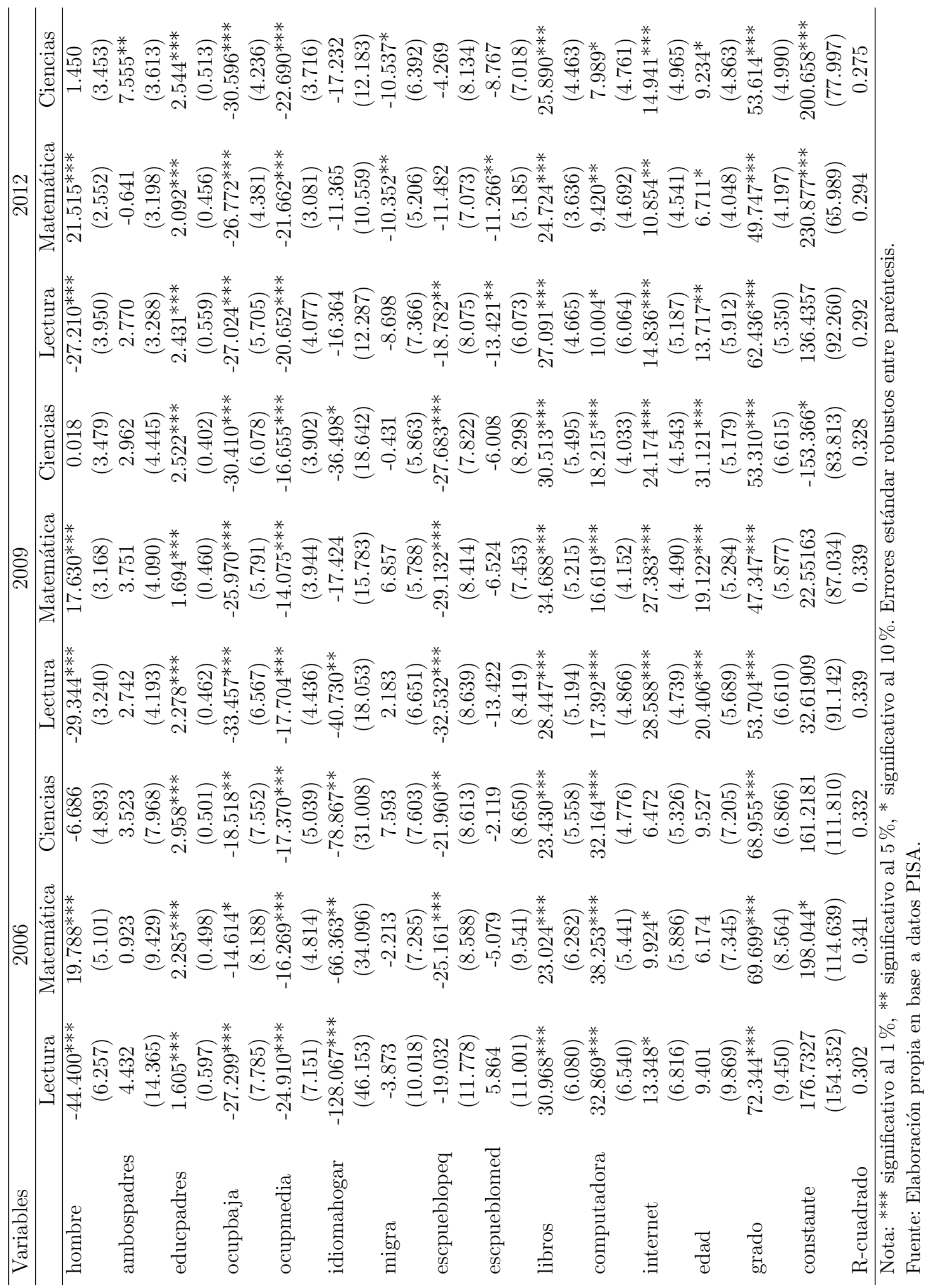


Tabla A1.10: Estimaciones del modelo de desempeño educativo por Efectos Fijos (2006, 2009 y 2012).

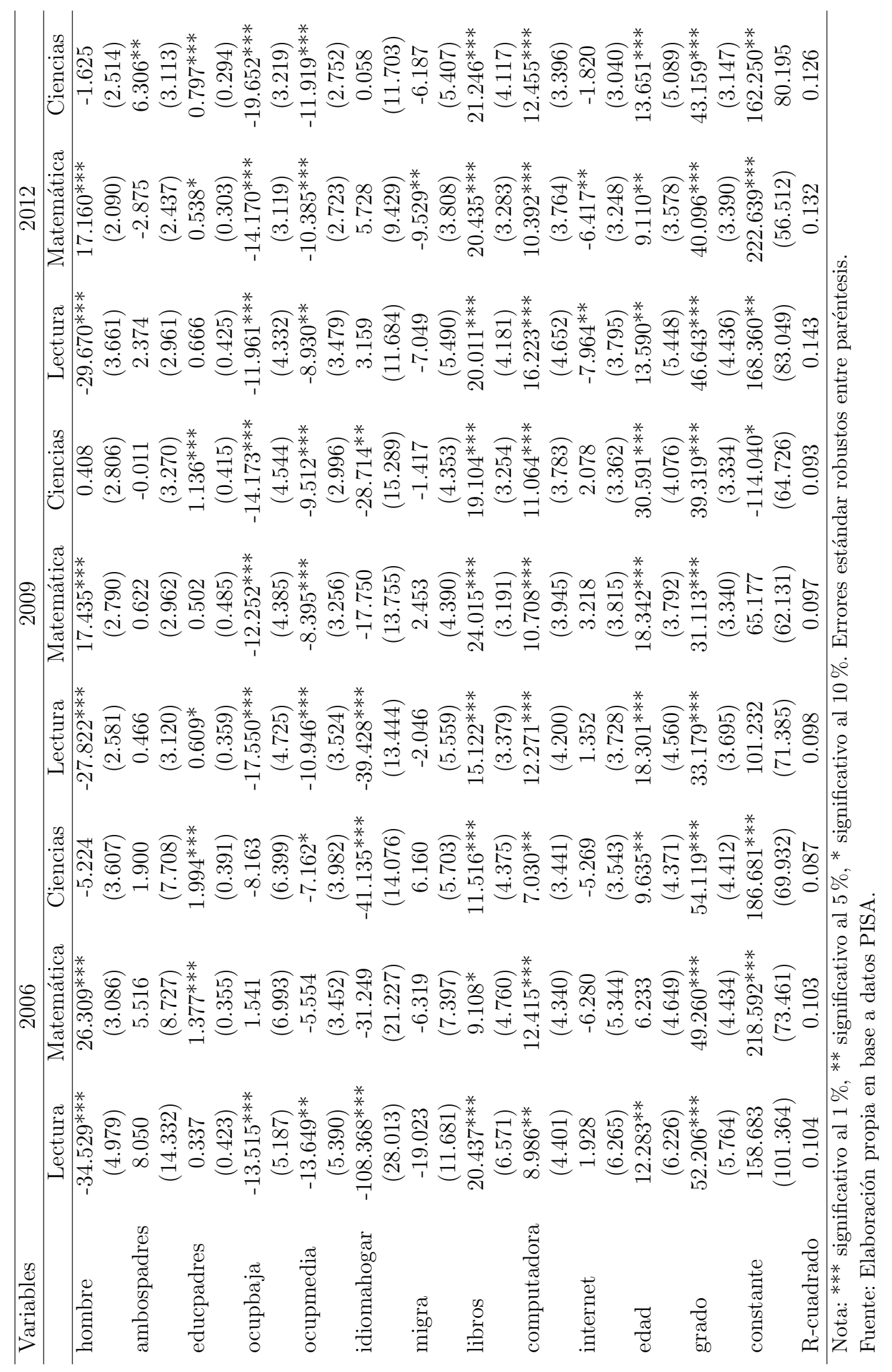


Tabla A1.11: Desigualdad de oportunidades en el acceso a educación de jóvenes de 15 años.

\begin{tabular}{|c|c|c|c|c|c|c|}
\hline \multirow{2}{*}{$\begin{array}{c}\text { Acceso a } \\
\text { Gini }\end{array}$} & \multicolumn{3}{|c|}{ Educación media } & \multicolumn{3}{|c|}{ Educación media privada } \\
\hline & Estimado & Interv & Conf. $95 \%$ & Estimado & Interv & onf. $95 \%$ \\
\hline 2003 & 0.067 & 0.062 & 0.076 & 0.375 & 0.360 & 0.389 \\
\hline 2004 & 0.067 & 0.062 & 0.072 & 0.342 & 0.328 & 0.353 \\
\hline 2005 & 0.043 & 0.041 & 0.046 & 0.352 & 0.343 & 0.366 \\
\hline 2006 & 0.058 & 0.053 & 0.060 & 0.338 & 0.329 & 0.348 \\
\hline 2007 & 0.053 & 0.049 & 0.058 & 0.388 & 0.374 & 0.408 \\
\hline 2008 & 0.062 & 0.057 & 0.068 & 0.334 & 0.325 & 0.344 \\
\hline 2009 & 0.049 & 0.047 & 0.053 & 0.379 & 0.369 & 0.395 \\
\hline 2010 & 0.047 & 0.044 & 0.051 & 0.385 & 0.370 & 0.401 \\
\hline 2011 & 0.045 & 0.041 & 0.048 & 0.366 & 0.352 & 0.381 \\
\hline 2012 & 0.052 & 0.049 & 0.058 & 0.420 & 0.404 & 0.433 \\
\hline Gini absoluto & Estimado & Interv & Conf. $95 \%$ & Estimado & Interv & onf. $95 \%$ \\
\hline 2003 & 0.059 & 0.055 & 0.064 & 0.095 & 0.091 & 0.100 \\
\hline 2004 & 0.058 & 0.054 & 0.062 & 0.100 & 0.096 & 0.107 \\
\hline 2005 & 0.039 & 0.037 & 0.043 & 0.103 & 0.096 & 0.109 \\
\hline 2006 & 0.052 & 0.049 & 0.055 & 0.106 & 0.102 & 0.112 \\
\hline 2007 & 0.046 & 0.042 & 0.052 & 0.112 & 0.104 & 0.118 \\
\hline 2008 & 0.055 & 0.052 & 0.059 & 0.096 & 0.092 & 0.100 \\
\hline 2009 & 0.045 & 0.042 & 0.047 & 0.108 & 0.103 & 0.114 \\
\hline 2010 & 0.043 & 0.039 & 0.046 & 0.120 & 0.115 & 0.128 \\
\hline 2011 & 0.041 & 0.038 & 0.043 & 0.106 & 0.100 & 0.115 \\
\hline 2012 & 0.047 & 0.043 & 0.051 & 0.114 & 0.107 & 0.119 \\
\hline Desvío estándar & Estimado & Interv & Conf. $95 \%$ & Estimado & Interv & onf. $95 \%$ \\
\hline 2003 & 0.117 & 0.110 & 0.127 & 0.173 & 0.160 & 0.179 \\
\hline 2004 & 0.117 & 0.109 & 0.127 & 0.180 & 0.174 & 0.189 \\
\hline 2005 & 0.080 & 0.074 & 0.086 & 0.188 & 0.176 & 0.198 \\
\hline 2006 & 0.104 & 0.098 & 0.113 & 0.190 & 0.183 & 0.199 \\
\hline 2007 & 0.090 & 0.082 & 0.099 & 0.199 & 0.190 & 0.206 \\
\hline 2008 & 0.110 & 0.101 & 0.121 & 0.172 & 0.166 & 0.179 \\
\hline 2009 & 0.088 & 0.084 & 0.093 & 0.192 & 0.185 & 0.201 \\
\hline 2010 & 0.085 & 0.078 & 0.092 & 0.214 & 0.204 & 0.225 \\
\hline 2011 & 0.081 & 0.076 & 0.086 & 0.192 & 0.182 & 0.209 \\
\hline 2012 & 0.097 & 0.086 & 0.104 & 0.207 & 0.197 & 0.218 \\
\hline Índice de Disimilitud & Estimado & Interv & Conf. $95 \%$ & Estimado & Interv & onf. $95 \%$ \\
\hline 2003 & 0.049 & 0.044 & 0.054 & 0.277 & 0.264 & 0.291 \\
\hline 2004 & 0.048 & 0.046 & 0.053 & 0.252 & 0.240 & 0.261 \\
\hline 2005 & 0.031 & 0.028 & 0.033 & 0.252 & 0.243 & 0.261 \\
\hline 2006 & 0.043 & 0.040 & 0.046 & 0.249 & 0.241 & 0.257 \\
\hline 2007 & 0.038 & 0.035 & 0.042 & 0.291 & 0.282 & 0.307 \\
\hline 2008 & 0.045 & 0.042 & 0.050 & 0.246 & 0.237 & 0.258 \\
\hline 2009 & 0.036 & 0.034 & 0.039 & 0.282 & 0.271 & 0.293 \\
\hline 2010 & 0.034 & 0.031 & 0.038 & 0.289 & 0.276 & 0.301 \\
\hline 2011 & 0.033 & 0.031 & 0.036 & 0.268 & 0.259 & 0.285 \\
\hline 2012 & 0.039 & 0.036 & 0.042 & 0.321 & 0.307 & 0.338 \\
\hline
\end{tabular}

Nota: Resultados encontrados a partir de las estimaciones del modelo no lineal Logit. Datos corresponden al segundo semestre de cada año. Intervalo de confianza estimado por bootstrap (100 reps.)

Fuente: Elaboración propia en base a EPH. 
Gráfico A1.1: Evolución de la desigualdad de oportunidades en el acceso a educación media (Panel a) y en el acceso a educación media privada (Panel b) por región.
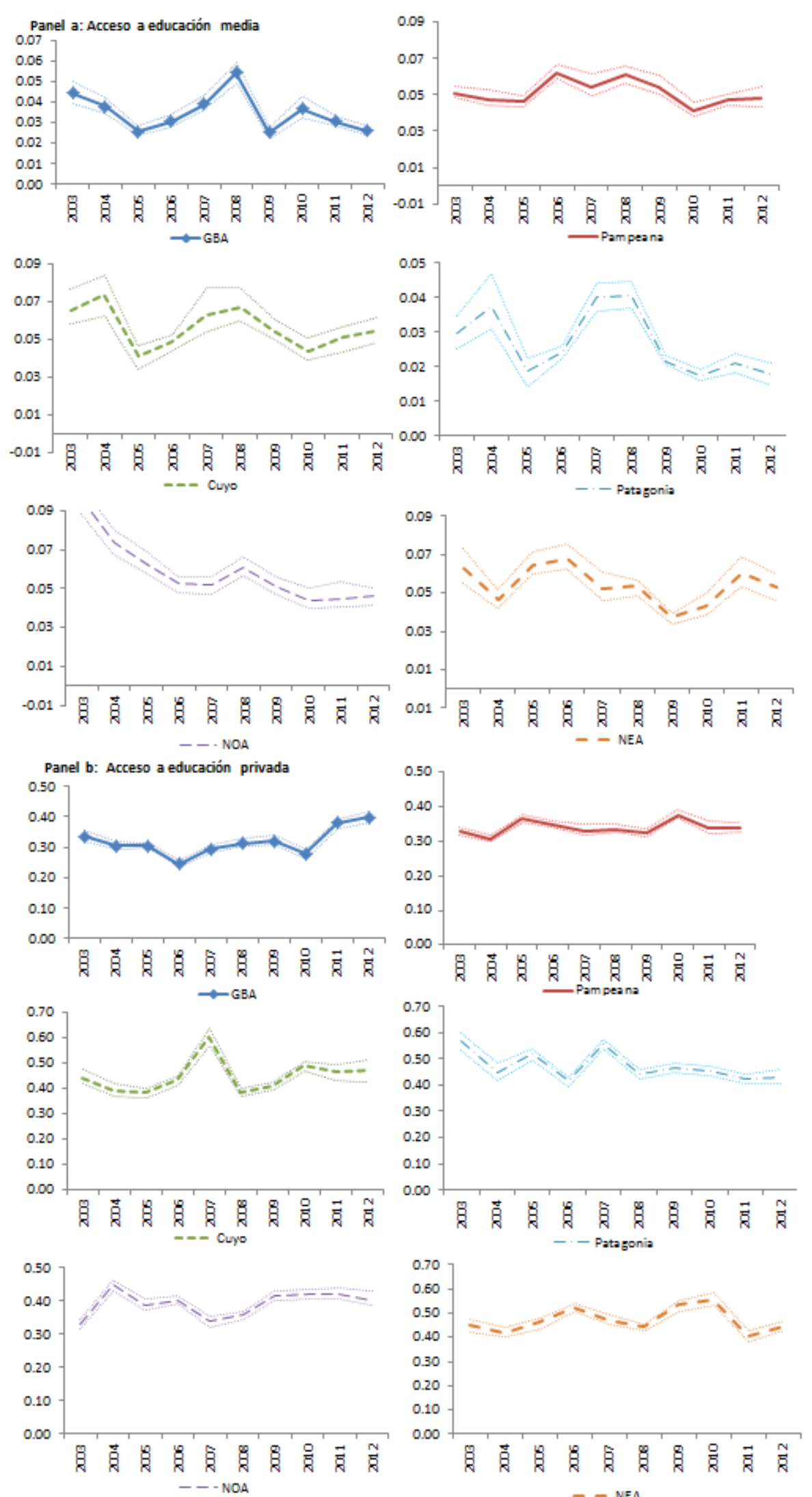
Tabla A1.12: Desigualdad de oportunidades en el acceso a calidad educativa según puntaje de la OCDE.

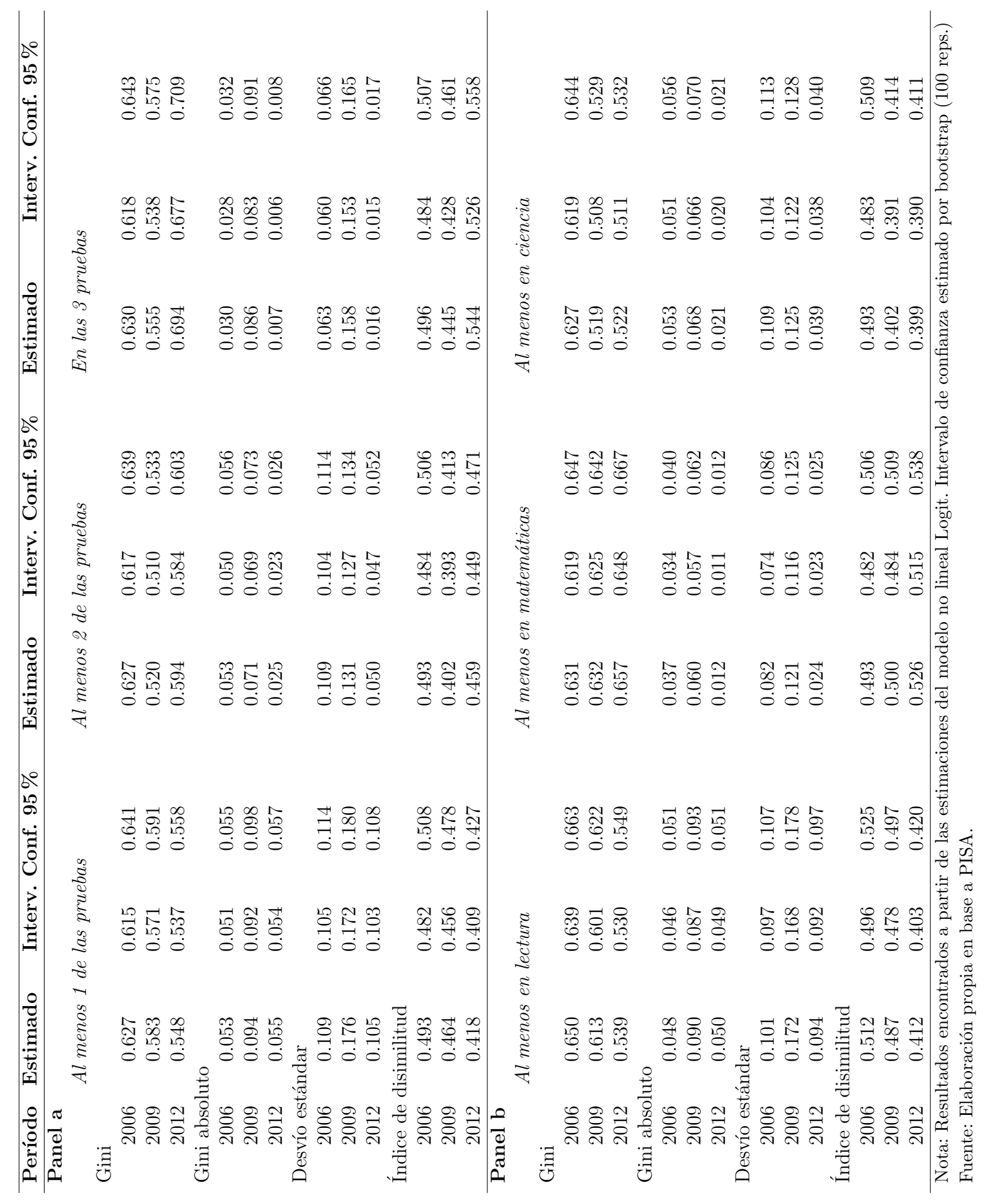


Tabla A1.13: Intervalos de confianza de desigualdad de oportunidades del desempeño educativo.

\begin{tabular}{|c|c|c|c|c|c|c|c|c|c|c|c|c|}
\hline \multirow{2}{*}{ Prueba } & \multicolumn{6}{|c|}{ MCO } & \multicolumn{6}{|c|}{ Efectos Fijos } \\
\hline & \multicolumn{2}{|c|}{ Lectura } & \multicolumn{2}{|c|}{ Matemática } & \multicolumn{2}{|c|}{ Ciencias } & \multicolumn{2}{|c|}{ Lectura } & \multicolumn{2}{|c|}{ Matemática } & \multicolumn{2}{|l|}{ Ciencias } \\
\hline Gini & Interv & Conf. 95\% & Interv. & Conf. $95 \%$ & Interv & Conf. $95 \%$ & Interv. & Conf. $95 \%$ & Interv. Conf. & $.95 \%$ & Interv. & Conf. $95 \%$ \\
\hline \multicolumn{13}{|c|}{ Parte a: Desigualdad de resultados } \\
\hline 2006 & 0.17 & 0.18 & 0.14 & 0.15 & 0.13 & 0.14 & 0.17 & 0.18 & 0.14 & 0.15 & 0.13 & 0.14 \\
\hline 2009 & 0.14 & 0.14 & 0.12 & 0.13 & 0.13 & 0.13 & 0.14 & 0.14 & 0.12 & 0.13 & 0.13 & 0.13 \\
\hline 2012 & 0.12 & 0.13 & 0.10 & 0.11 & 0.11 & 0.11 & 0.12 & 0.13 & 0.10 & 0.11 & 0.11 & 0.11 \\
\hline \multicolumn{13}{|c|}{ Parte b: Desigualdad de oportunidades } \\
\hline 2006 & 0.28 & 0.30 & 0.07 & 0.08 & 0.19 & 0.20 & 0.05 & 0.06 & 0.03 & 0.03 & 0.02 & 0.02 \\
\hline 2009 & 0.06 & 0.07 & 0.06 & 0.06 & 0.06 & 0.06 & 0.04 & 0.04 & 0.03 & 0.03 & 0.02 & 0.02 \\
\hline 2012 & 0.06 & 0.06 & 0.04 & 0.04 & 0.04 & 0.04 & 0.02 & 0.03 & 0.02 & 0.02 & 0.02 & 0.02 \\
\hline \multicolumn{13}{|c|}{ Parte c: Contribución de la desigualdad de oportunidades } \\
\hline 2006 & $164 \%$ & $174 \%$ & $52 \%$ & $54 \%$ & $137 \%$ & $144 \%$ & $32 \%$ & $34 \%$ & $23 \%$ & $24 \%$ & $14 \%$ & $14 \%$ \\
\hline 2009 & $47 \%$ & $49 \%$ & $48 \%$ & $50 \%$ & $42 \%$ & $44 \%$ & $25 \%$ & $27 \%$ & $22 \%$ & $23 \%$ & $16 \%$ & $17 \%$ \\
\hline 2012 & $48 \%$ & $50 \%$ & $35 \%$ & $37 \%$ & $33 \%$ & $34 \%$ & $20 \%$ & $21 \%$ & $19 \%$ & $20 \%$ & $18 \%$ & $18 \%$ \\
\hline Gini absoluto & Interv & Conf. 95\% & Interv. & Conf. $95 \%$ & Interv & Conf. $95 \%$ & Interv. & Conf. $95 \%$ & Interv. Conf & $95 \%$ & Interv. & Conf. $95 \%$ \\
\hline \multicolumn{13}{|c|}{ Parte a: Desigualdad de resultados } \\
\hline 2006 & 64.83 & 68.36 & 53.17 & 57.01 & 53.11 & 56.24 & 64.83 & 68.36 & 53.17 & 57.01 & 53.11 & 56.24 \\
\hline 2009 & 55.83 & 58.47 & 49.24 & 51.76 & 53.06 & 55.45 & 55.83 & 58.47 & 49.24 & 51.76 & 53.06 & 55.45 \\
\hline 2012 & 50.32 & 52.81 & 40.63 & 42.40 & 45.15 & 47.00 & 50.32 & 52.81 & 40.63 & 42.40 & 45.15 & 47.00 \\
\hline \multicolumn{13}{|c|}{ Parte b: Desigualdad de oportunidades } \\
\hline 2006 & 24.30 & 25.55 & 23.09 & 24.13 & 20.79 & 21.59 & 12.55 & 13.36 & 9.99 & 10.49 & 7.89 & 8.25 \\
\hline 2009 & 26.11 & 27.13 & 23.79 & 24.82 & 23.94 & 24.91 & 11.32 & 11.87 & 9.32 & 9.75 & 9.15 & 9.58 \\
\hline 2012 & 17.70 & 18.57 & 15.41 & 16.16 & 15.92 & 16.50 & 10.35 & 10.80 & 8.40 & 8.86 & 8.49 & 8.97 \\
\hline \multicolumn{13}{|c|}{ Parte c: Contribución de la desigualdad de oportunidades } \\
\hline 2006 & $36 \%$ & $38 \%$ & $42 \%$ & $44 \%$ & $38 \%$ & $39 \%$ & $19 \%$ & $20 \%$ & $18 \%$ & $19 \%$ & $14 \%$ & $15 \%$ \\
\hline 2009 & $46 \%$ & $47 \%$ & $47 \%$ & $49 \%$ & $44 \%$ & $46 \%$ & $20 \%$ & $21 \%$ & $18 \%$ & $19 \%$ & $17 \%$ & $18 \%$ \\
\hline 2012 & $34 \%$ & $36 \%$ & $37 \%$ & $39 \%$ & $35 \%$ & $36 \%$ & $20 \%$ & $21 \%$ & $20 \%$ & $21 \%$ & $18 \%$ & $19 \%$ \\
\hline Desvío estándar & Interv & Conf. $95 \%$ & Interv. & Conf. $95 \%$ & Interv & Conf. $95 \%$ & Interv. & Conf. $95 \%$ & Interv. Conf. & $.95 \%$ & Interv. & Conf. $95 \%$ \\
\hline \multicolumn{13}{|c|}{ Parte a: Desigualdad de resultados } \\
\hline 2006 & 115.85 & 123.79 & 95.60 & 102.02 & 94.50 & 100.36 & 115.85 & 123.79 & 95.60 & 102.02 & 94.50 & 100.36 \\
\hline 2009 & 99.24 & 104.93 & 88.22 & 92.31 & 94.66 & 98.48 & 99.24 & 104.93 & 88.22 & 92.31 & 94.66 & 98.48 \\
\hline 2012 & 89.65 & 93.40 & 71.47 & 75.47 & 79.47 & 83.50 & 89.65 & 93.40 & 71.47 & 75.47 & 79.47 & 83.50 \\
\hline \multicolumn{13}{|c|}{ Parte b: Desigualdad de oportunidades } \\
\hline 2006 & 43.28 & 45.24 & 40.83 & 42.07 & 36.26 & 37.94 & 22.69 & 24.47 & 17.59 & 18.27 & 13.87 & 14.51 \\
\hline 2009 & 45.68 & 47.52 & 41.44 & 43.53 & 42.24 & 43.98 & 20.13 & 20.97 & 16.62 & 17.37 & 16.17 & 16.88 \\
\hline 2012 & 31.27 & 32.53 & 27.08 & 28.37 & 27.78 & 29.00 & 18.35 & 19.01 & 14.94 & 15.62 & 15.06 & 15.89 \\
\hline \multicolumn{13}{|c|}{ Parte c: Contribución de la desigualdad de oportunidades } \\
\hline 2006 & $37 \%$ & $38 \%$ & $42 \%$ & $43 \%$ & $37 \%$ & $39 \%$ & $19 \%$ & $21 \%$ & $18 \%$ & $19 \%$ & $14 \%$ & $15 \%$ \\
\hline 2009 & $45 \%$ & $47 \%$ & $46 \%$ & $48 \%$ & $44 \%$ & $46 \%$ & $20 \%$ & $21 \%$ & $19 \%$ & $19 \%$ & $17 \%$ & $17 \%$ \\
\hline 2012 & $34 \%$ & $36 \%$ & $37 \%$ & $39 \%$ & $34 \%$ & $35 \%$ & $20 \%$ & $21 \%$ & $20 \%$ & $21 \%$ & $18 \%$ & $19 \%$ \\
\hline
\end{tabular}

Fuente: Elaboración propia en base a PISA. 


\section{Apéndice 2: Capítulo 2}

\section{Estadísticas descriptivas.}

Tabla A2.1: Estadísticas descriptivas de PISA. Parte 1.

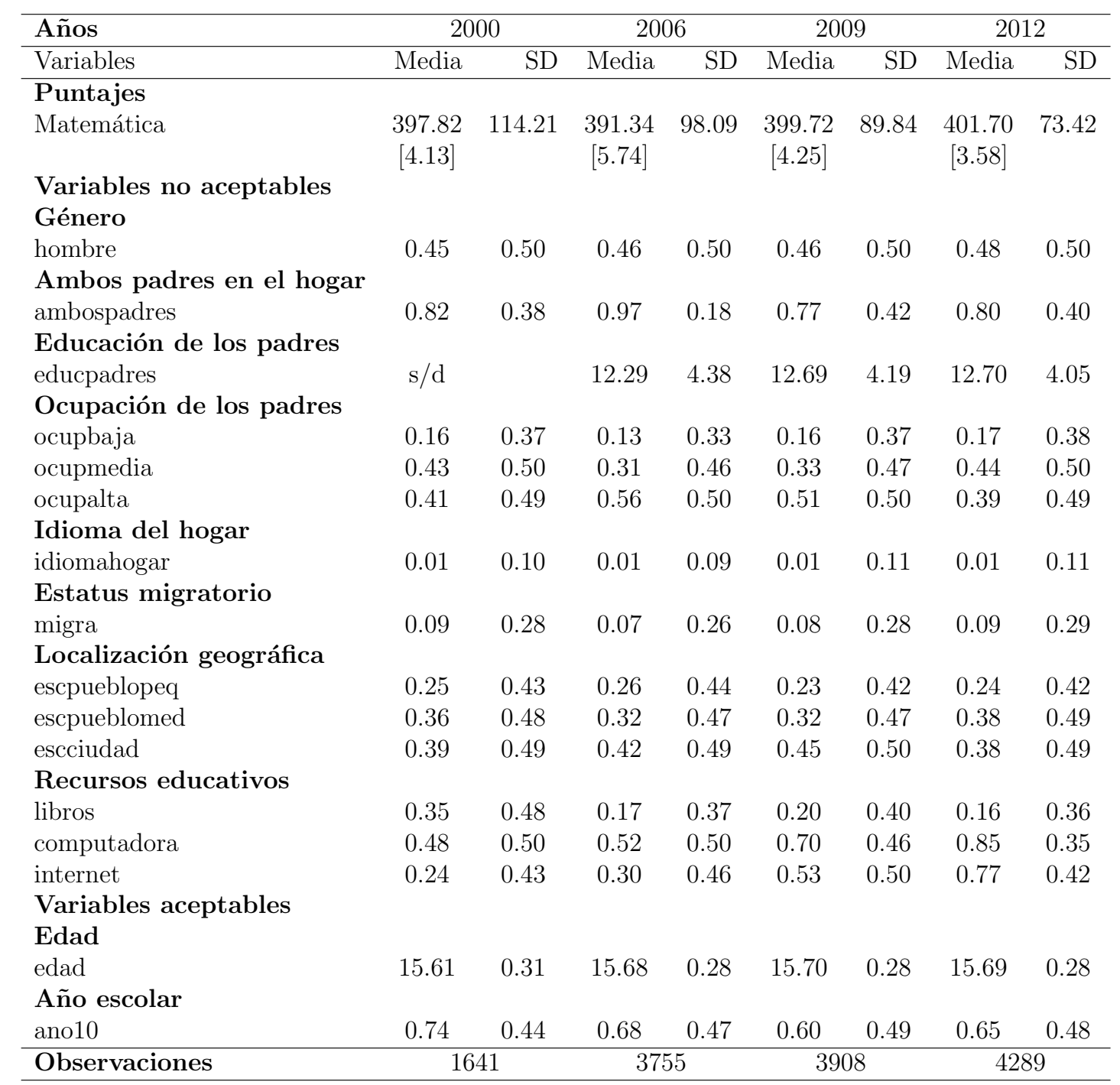

Nota: SD: desvío estándar. Errores estándar entre corchetes.

Fuente: Elaboración propia en base a datos PISA. 
Table A2.1 (cont.): Estadísticas descriptivas de PISA. Parte 2.

\begin{tabular}{|c|c|c|c|c|c|c|c|c|}
\hline \multirow{2}{*}{\begin{tabular}{|l|} 
Años \\
Variables
\end{tabular}} & \multicolumn{2}{|c|}{2000} & \multicolumn{2}{|c|}{2006} & \multicolumn{2}{|c|}{2009} & \multicolumn{2}{|c|}{2012} \\
\hline & Media & SD & Media & SD & Media & SD & Media & SD \\
\hline Puntajes & & & & & & & & \\
\hline Matemática & $\begin{array}{l}403.31 \\
{[4.10]}\end{array}$ & 110.81 & $\begin{array}{c}396.05 \\
{[2.10]}\end{array}$ & 95.85 & $\begin{array}{l}417.57 \\
{[1.88]}\end{array}$ & 86.93 & $\begin{array}{l}404.55 \\
{[1.62]}\end{array}$ & 72.34 \\
\hline $\begin{array}{l}\text { Variables no aceptables } \\
\text { Género }\end{array}$ & & & & & & & & \\
\hline hombre & 0.44 & 0.50 & 0.46 & 0.50 & 0.45 & 0.50 & 0.47 & 0.50 \\
\hline $\begin{array}{l}\text { Ambos padres en el hogar } \\
\text { ambospadres }\end{array}$ & 0.82 & 0.38 & 0.97 & 0.18 & 0.77 & 0.42 & 0.81 & 0.39 \\
\hline $\begin{array}{l}\text { Educación de los padres } \\
\text { educpadres }\end{array}$ & $\mathrm{s} / \mathrm{d}$ & & 12.34 & 4.35 & 12.94 & 4.17 & 12.70 & 4.08 \\
\hline $\begin{array}{l}\text { Ocupación de los padres } \\
\text { ocupbaja }\end{array}$ & 0.16 & 0.37 & 0.12 & 0.32 & 0.14 & 0.35 & 0.16 & 0.36 \\
\hline ocupmedia & 0.42 & 0.49 & 0.31 & 0.46 & 0.30 & 0.46 & 0.45 & 0.50 \\
\hline ocupalta & 0.41 & 0.49 & 0.57 & 0.50 & 0.56 & 0.50 & 0.39 & 0.49 \\
\hline $\begin{array}{l}\text { Idioma del hogar } \\
\text { idiomahogar }\end{array}$ & 0.01 & 0.10 & 0.01 & 0.08 & 0.01 & 0.10 & 0.01 & 0.11 \\
\hline $\begin{array}{l}\text { Estatus migratorio } \\
\text { migra }\end{array}$ & 0.09 & 0.28 & 0.07 & 0.26 & 0.08 & 0.27 & 0.09 & 0.29 \\
\hline $\begin{array}{l}\text { Localización geográfica } \\
\text { escpueblopeq }\end{array}$ & 0.25 & 0.43 & 0.25 & 0.44 & 0.20 & 0.40 & 0.23 & 0.42 \\
\hline escpueblomed & 0.36 & 0.48 & 0.32 & 0.47 & 0.33 & 0.47 & 0.38 & 0.49 \\
\hline escciudad & 0.39 & 0.49 & 0.42 & 0.49 & 0.47 & 0.50 & 0.39 & 0.49 \\
\hline Recursos educa & & & & & & & & \\
\hline libros & 0.37 & 0.48 & 0.17 & 0.38 & 0.23 & 0.42 & 0.15 & 0.36 \\
\hline computadora & 0.49 & 0.50 & 0.53 & 0.50 & 0.75 & 0.43 & 0.85 & 0.35 \\
\hline internet & 0.25 & 0.43 & 0.31 & 0.46 & 0.59 & 0.49 & 0.76 & 0.43 \\
\hline $\begin{array}{l}\text { Contexto del colegio } \\
\text { educación promedio padres del colegio } \\
\text { Edad }\end{array}$ & $\mathrm{s} / \mathrm{d}$ & & 12.28 & 2.17 & 12.88 & 2.21 & 12.74 & 2.03 \\
\hline edad & 15.61 & 0.31 & 15.68 & 0.28 & 15.70 & 0.28 & 15.70 & 0.28 \\
\hline Tiempo que dedica a matemáticas & fuera $\mathrm{c}$ & el coleg & & & & & & \\
\hline tiempomath & 0.58 & 0.49 & 0.13 & 0.34 & 0.16 & 0.37 & 0.15 & 0.35 \\
\hline $\begin{array}{l}\text { Año escolar } \\
\text { ano10 }\end{array}$ & 0.75 & 0.43 & 0.70 & 0.46 & 0.66 & 0.47 & 0.65 & 0.48 \\
\hline
\end{tabular}

Nota: SD: desvío estándar. Errores estándar entre corchetes.

Fuente: Elaboración propia en base a datos PISA. 
Tabla A2.2: Desigualdad de los puntajes de matemáticas.

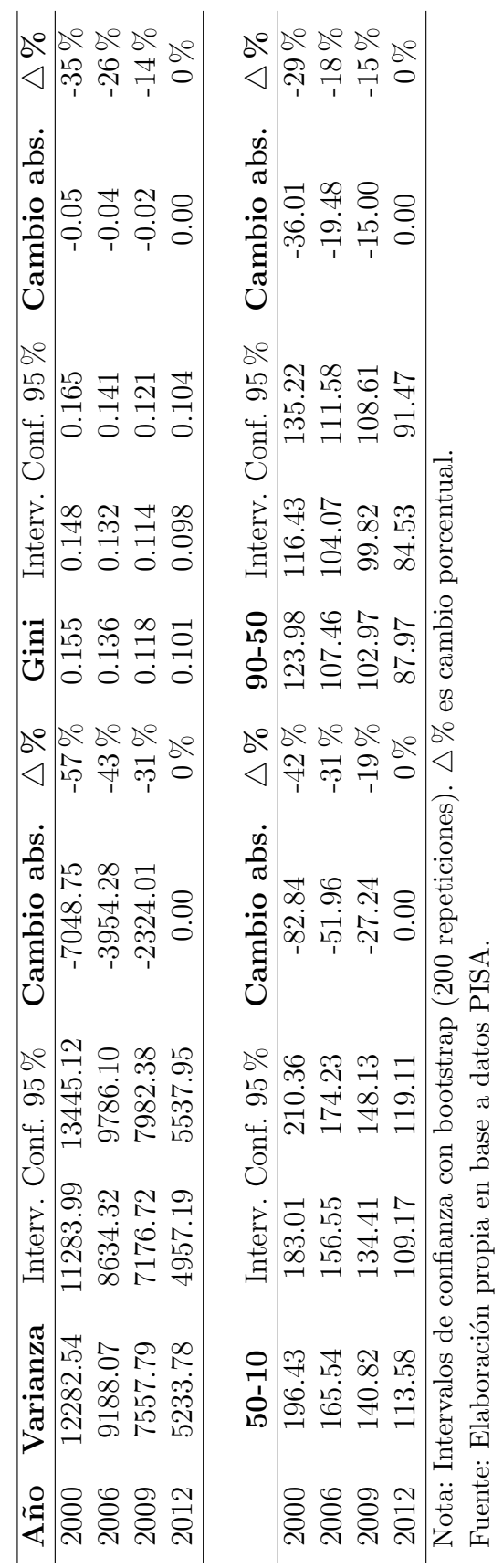


Resultados de regresiones de RIF.

Tabla A2.3: Estimaciones de las regresiones de MCO y de RIF. Modelos 2 y 3.

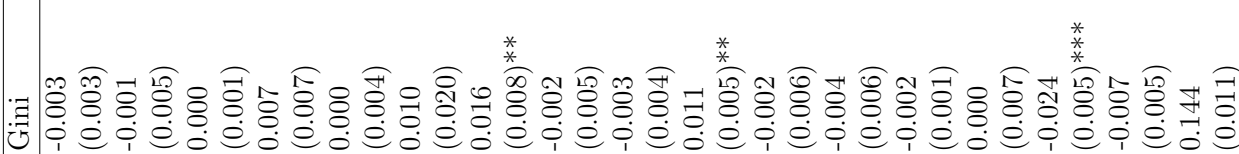

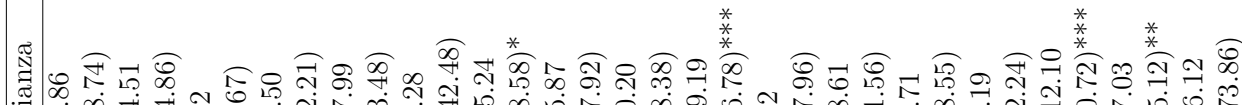

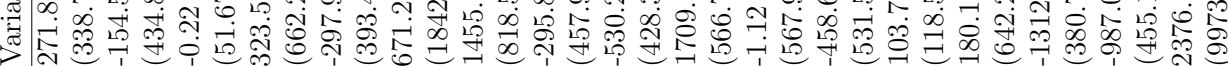

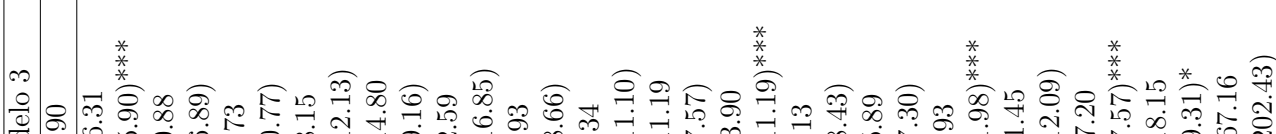

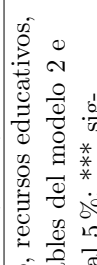

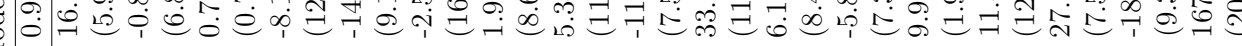

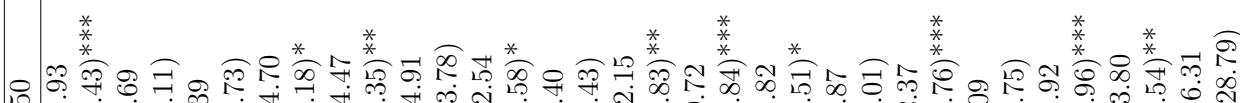

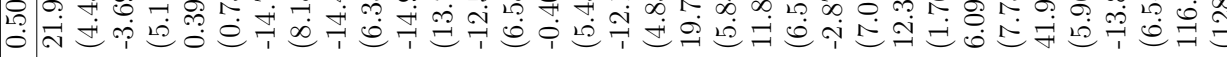

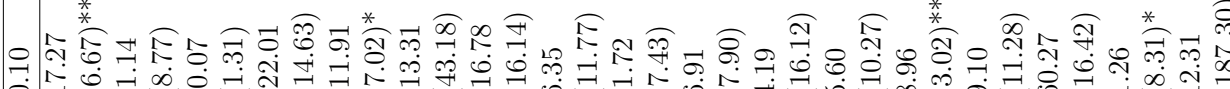
:

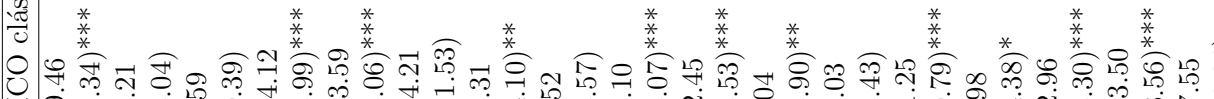

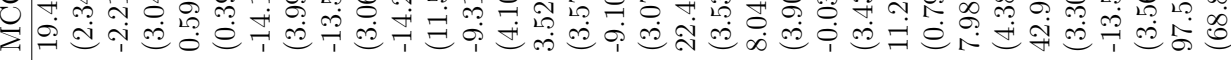

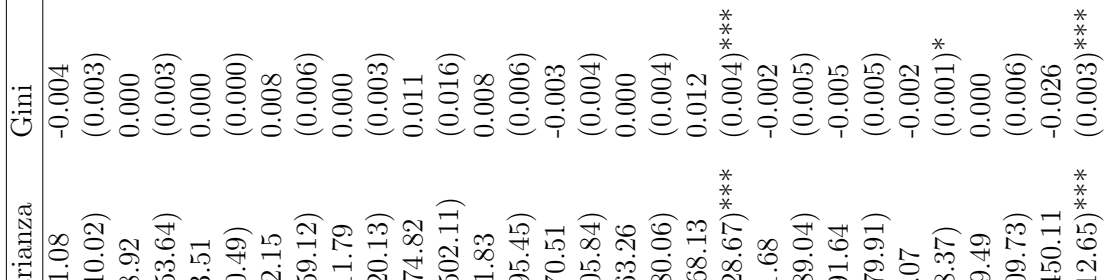

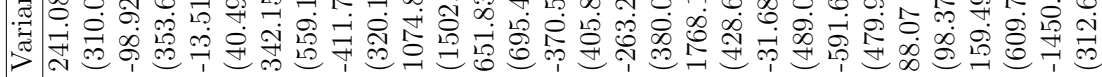

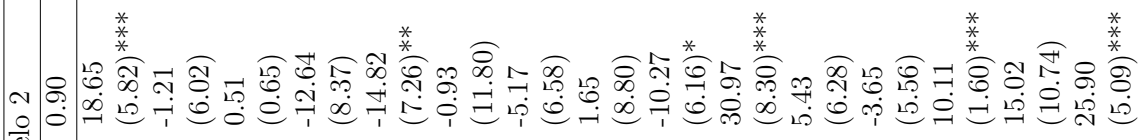

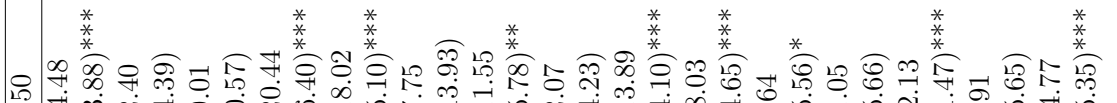

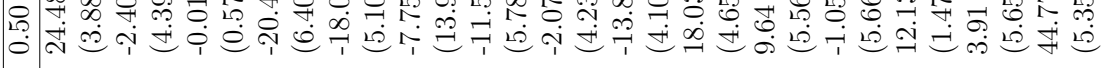

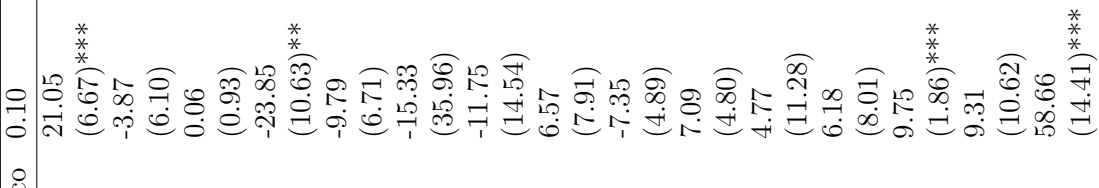

ठ

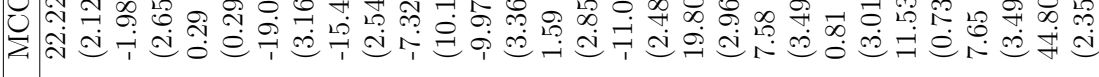

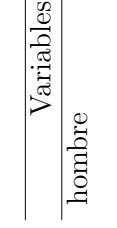
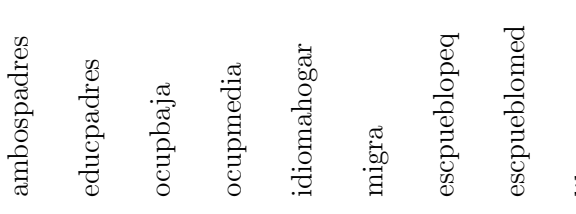

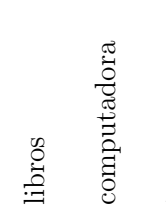

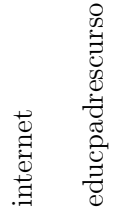

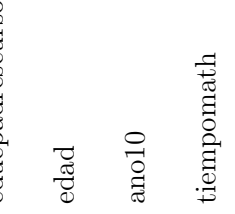

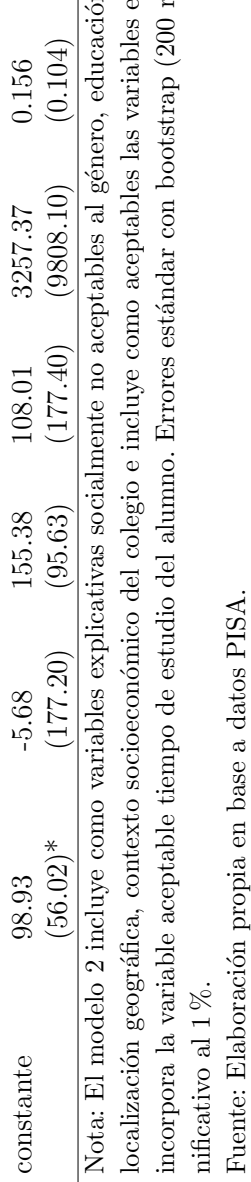




\section{Resultados de la descomposición de RIF.}

Tabla A2.4: Descomposición de la desigualdad del desempeño educativo (2000-2012).

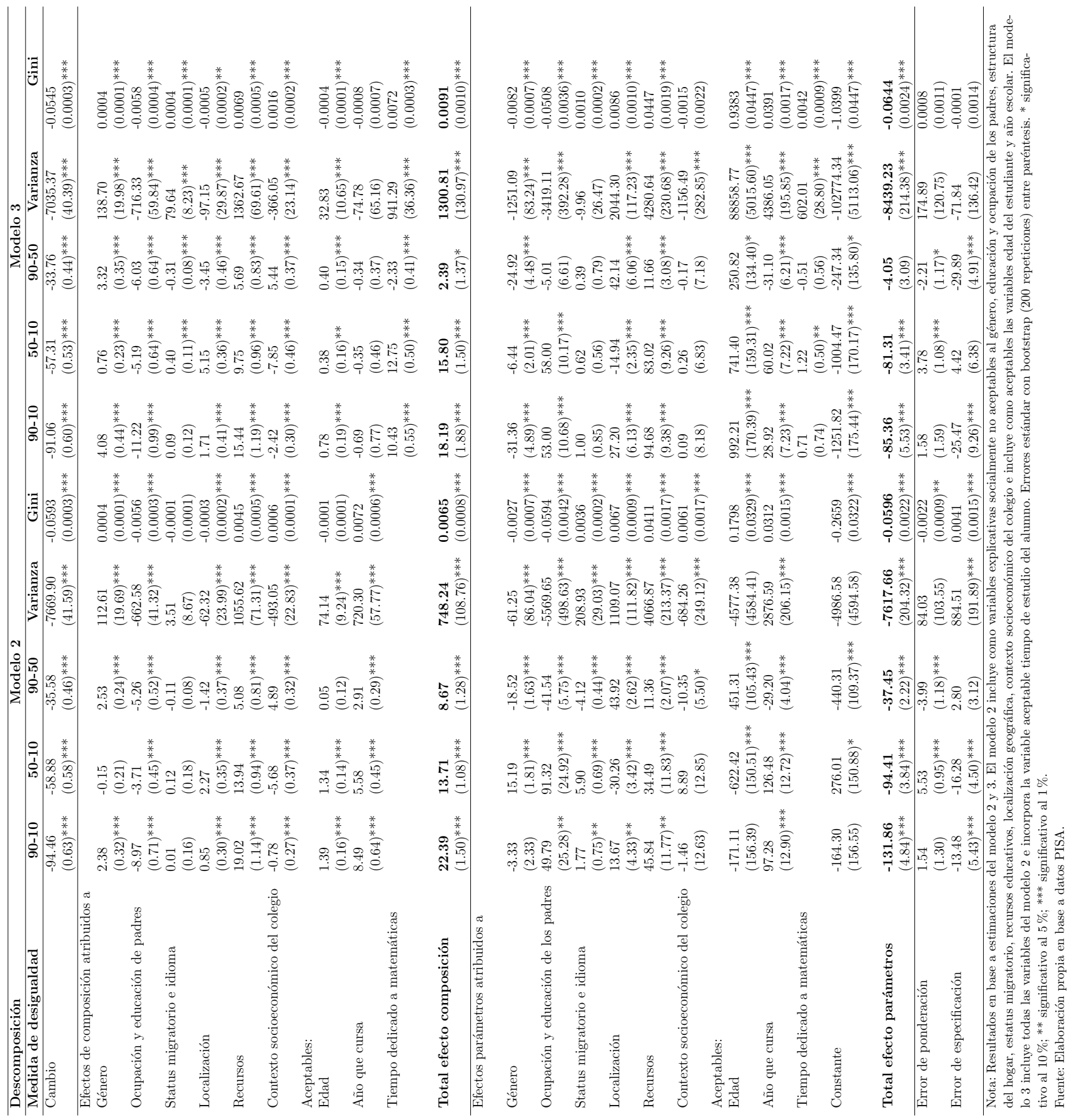


Tabla A2.5: Desigualdad de los puntajes de matemáticas en países de América Latina.

\begin{tabular}{|c|c|c|c|c|c|c|c|c|c|c|}
\hline Brasil & Varianza & Inferior & Superior & Cambio abs. & $\triangle \%$ & Gini & Inferior & Superior & Cambio abs. & $\triangle \%$ \\
\hline 2000 & 9058.90 & 8482.27 & 9586.18 & -3183.83 & $-35 \%$ & 0.16 & 0.15 & 0.16 & -0.05 & $-32 \%$ \\
\hline 2006 & 7990.02 & 7609.10 & 8403.50 & -2114.95 & $-26 \%$ & 0.13 & 0.13 & 0.13 & -0.03 & $-19 \%$ \\
\hline 2009 & 6821.67 & 6597.76 & 7188.57 & -946.59 & $-14 \%$ & 0.11 & 0.11 & 0.11 & -0.01 & $-5 \%$ \\
\hline \multirow[t]{2}{*}{2012} & 5875.08 & 5604.06 & 6063.58 & 0.00 & $0 \%$ & 0.11 & 0.10 & 0.11 & 0.00 & $0 \%$ \\
\hline & $50-10$ & Inferior & Superior & Cambio abs. & $\triangle \%$ & $90-50$ & Inferior & Superior & Cambio abs. & $\triangle \%$ \\
\hline 2000 & 145.59 & 140.14 & 152.30 & -38.75 & $-27 \%$ & 119.56 & 112.83 & 125.77 & -23.83 & $-20 \%$ \\
\hline 2006 & 132.62 & 127.82 & 138.06 & -25.78 & $-19 \%$ & 106.07 & 103.00 & 109.62 & -10.34 & $-10 \%$ \\
\hline 2009 & 122.07 & 117.56 & 126.22 & -15.23 & $-12 \%$ & 101.50 & 98.15 & 105.34 & -5.77 & $-6 \%$ \\
\hline 2012 & 106.84 & 104.06 & 109.34 & 0.00 & $0 \%$ & 95.73 & 92.33 & 98.63 & 0.00 & $0 \%$ \\
\hline Chile & Varianza & Inferior & Superior & Cambio abs. & $\triangle \%$ & Gini & Inferior & Superior & Cambio abs. & $\triangle \%$ \\
\hline 2000 & 8232.98 & 7836.06 & 8746.08 & -1879.03 & $-23 \%$ & 0.13 & 0.13 & 0.14 & -0.03 & $-20 \%$ \\
\hline 2006 & 7483.77 & 7240.20 & 7789.93 & -1129.82 & $-15 \%$ & 0.12 & 0.12 & 0.12 & -0.01 & $-11 \%$ \\
\hline 2009 & 5699.57 & 5461.23 & 5934.03 & 654.37 & $11 \%$ & 0.10 & 0.09 & 0.10 & 0.01 & $10 \%$ \\
\hline \multirow[t]{2}{*}{2012} & 6353.95 & 6118.38 & 6583.83 & 0.00 & $0 \%$ & 0.10 & 0.10 & 0.11 & 0.00 & $0 \%$ \\
\hline & $50-10$ & Inferior & Superior & Cambio abs. & $\triangle \%$ & $90-50$ & Inferior & Superior & Cambio abs. & $\triangle \%$ \\
\hline 2000 & 157.49 & 151.40 & 164.82 & -42.18 & $-27 \%$ & 103.01 & 98.86 & 107.49 & -1.33 & $-1 \%$ \\
\hline 2006 & 131.09 & 127.26 & 136.66 & -15.78 & $-12 \%$ & 106.41 & 103.46 & 110.60 & -4.73 & $-4 \%$ \\
\hline 2009 & 117.45 & 113.43 & 120.90 & -2.14 & $-2 \%$ & 91.18 & 88.50 & 94.51 & 10.50 & $12 \%$ \\
\hline 2012 & 115.31 & 112.05 & 120.21 & 0.00 & $0 \%$ & 101.67 & 97.74 & 105.08 & 0.00 & $0 \%$ \\
\hline México & Varianza & Inferior & Superior & Cambio abs. & $\triangle \%$ & Gini & Inferior & Superior & Cambio abs. & $\triangle \%$ \\
\hline 2000 & 6425.13 & 6113.23 & 6835.71 & -1312.44 & $-20 \%$ & 0.11 & 0.11 & 0.12 & -0.02 & $-18 \%$ \\
\hline 2006 & 6381.91 & 6179.72 & 6589.85 & -1269.22 & $-20 \%$ & 0.11 & 0.11 & 0.11 & -0.01 & $-13 \%$ \\
\hline 2009 & 5986.92 & 5825.08 & 6187.84 & -874.23 & $-15 \%$ & 0.10 & 0.10 & 0.10 & 0.00 & $-4 \%$ \\
\hline \multirow[t]{2}{*}{2012} & 5112.69 & 4985.61 & 5247.44 & 0.00 & $0 \%$ & 0.09 & 0.09 & 0.10 & 0.00 & $0 \%$ \\
\hline & $50-10$ & Inferior & Superior & Cambio abs. & $\triangle \%$ & $90-50$ & Inferior & Superior & Cambio abs. & $\triangle \%$ \\
\hline 2000 & 128.78 & 123.45 & 135.45 & -19.90 & $-15 \%$ & 95.95 & 91.65 & 99.77 & -9.12 & $-10 \%$ \\
\hline 2006 & 125.64 & 122.07 & 129.57 & -16.76 & $-13 \%$ & 95.53 & 93.40 & 97.88 & -8.70 & $-9 \%$ \\
\hline 2009 & 128.75 & 124.80 & 131.45 & -19.87 & $-15 \%$ & 90.44 & 88.00 & 92.30 & -3.61 & $-4 \%$ \\
\hline 2012 & 108.88 & 106.18 & 111.00 & 0.00 & $0 \%$ & 86.83 & 84.88 & 88.63 & 0.00 & $0 \%$ \\
\hline
\end{tabular}

Nota: Intervalos de confianza con bootstrap (200 repeticiones). $\triangle \%$ es cambio porcentual

Fuente: Elaboración propia en base a datos PISA. 
Tabla A2.6: Descomposición de la desigualdad de oportunidades del desempeño educativo de países de América Latina (2000-2012).

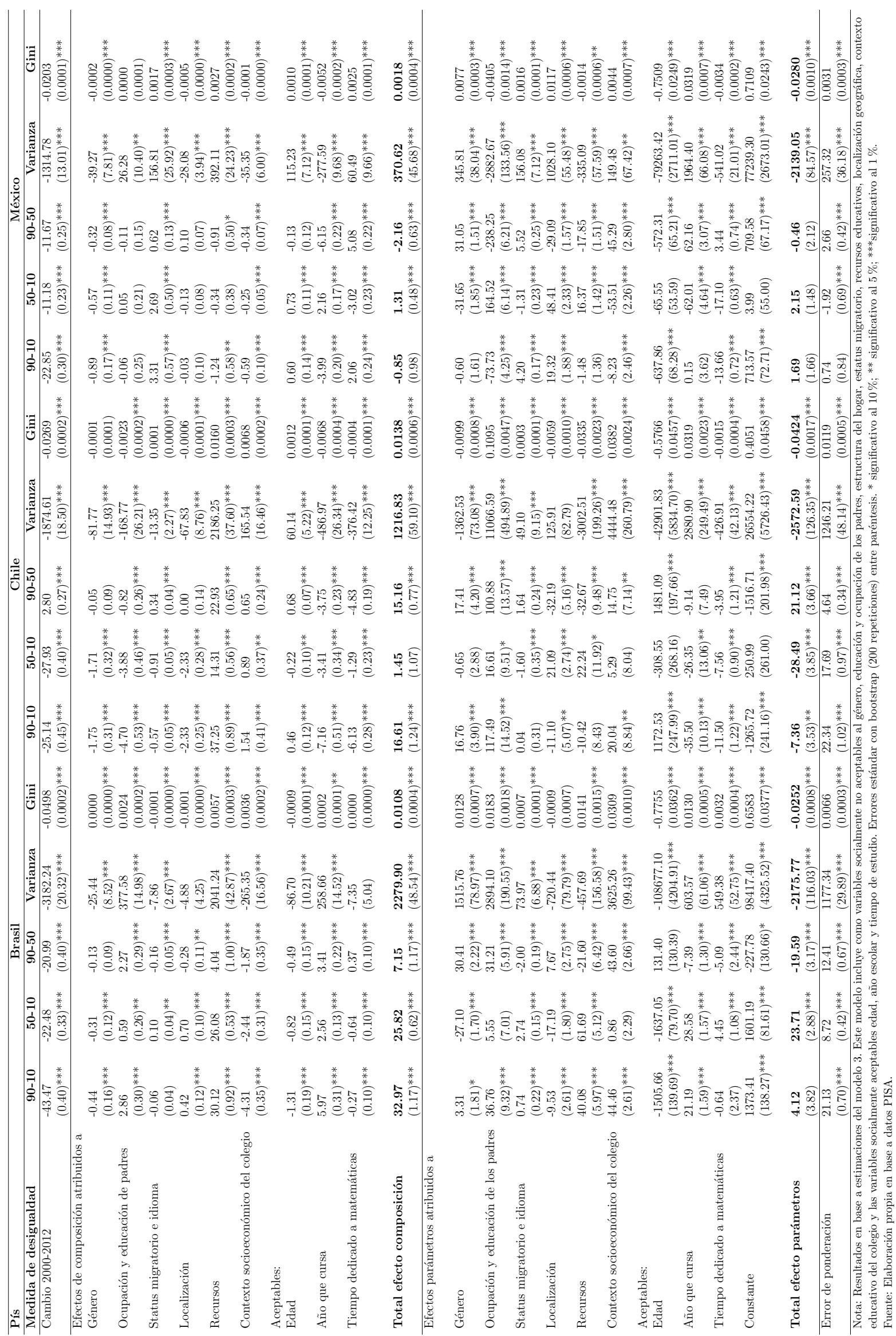




\section{Apéndice 3: Capítulo 3}

\section{Estadísticas descriptivas.}

Tabla A3.1: Puntajes promedios de las pruebas PISA según circunstancias del alumno.

\begin{tabular}{|c|c|c|c|c|}
\hline \multirow{2}{*}{$\begin{array}{l}\text { Pomedio puntajes } \\
\text { pruebas PISA }\end{array}$} & \multicolumn{3}{|c|}{ Tipo de establecimiento } & \multirow{2}{*}{ Total } \\
\hline & Público & Privado subvencionado & Privado independiente & \\
\hline \multicolumn{5}{|c|}{ Según máximo nivel educativo alcanzado por los padres } \\
\hline \multicolumn{5}{|l|}{ Alto } \\
\hline \multirow[t]{2}{*}{ Matemática } & 380 & 442 & 452 & 410 \\
\hline & {$[3.25]$} & {$[3.93]$} & {$[10.13]$} & {$[2.82]$} \\
\hline \multirow{2}{*}{ Lectura } & 380 & 458 & 462 & 417 \\
\hline & {$[4.00]$} & {$[5.41]$} & {$[11.52]$} & {$[3.32]$} \\
\hline \multirow[t]{2}{*}{ Ciencia } & 396 & 463 & 466 & 427 \\
\hline & {$[3.54]$} & {$[4.09]$} & {$[9.58]$} & {$[2.93]$} \\
\hline \multicolumn{5}{|l|}{ Medio } \\
\hline \multirow[t]{2}{*}{ Matemática } & 363 & 426 & 424 & 382 \\
\hline & {$[3.05]$} & {$[6.23]$} & {$[14.04]$} & {$[3.21]$} \\
\hline \multirow[t]{2}{*}{ Lectura } & 358 & 437 & 436 & 382 \\
\hline & {$[4.09]$} & {$[5.84]$} & {$[12.15]$} & {$[3.66]$} \\
\hline \multirow[t]{2}{*}{ Ciencia } & 372 & 442 & 439 & 393 \\
\hline & {$[3.26]$} & {$[5.76]$} & {$[11.90]$} & {$[3.26]$} \\
\hline \multicolumn{5}{|l|}{ Bajo } \\
\hline \multirow{2}{*}{ Matemática } & 337 & 374 & 370 & 344 \\
\hline & {$[3.37]$} & {$[7.08]$} & {$[9.60]$} & {$[2.94]$} \\
\hline \multirow{2}{*}{ Lectura } & 337 & 381 & 380 & 345 \\
\hline & {$[3.73]$} & {$[13.79]$} & [14.81] & {$[3.34]$} \\
\hline \multirow[t]{2}{*}{ Ciencia } & 346 & 391 & 384 & 354 \\
\hline & {$[3.29]$} & {$[10.20]$} & [11.63] & {$[3.09]$} \\
\hline \multirow{2}{*}{\multicolumn{5}{|c|}{$\begin{array}{l}\text { Según nivel socioeconómico cultural familiar } \\
\text { Alto }\end{array}$}} \\
\hline & & & & \\
\hline \multirow[t]{2}{*}{ Matemática } & 405 & 450 & 464 & 433 \\
\hline & {$[3.95]$} & [3.88] & [10.09] & {$[2.84]$} \\
\hline \multirow[t]{2}{*}{ Lectura } & 404 & 465 & 474 & 440 \\
\hline & {$[4.59]$} & {$[5.60]$} & {$[12.33]$} & {$[3.46]$} \\
\hline \multirow[t]{2}{*}{ Ciencia } & 420 & 470 & 478 & 450 \\
\hline & {$[4.51]$} & {$[3.90]$} & {$[9.36]$} & {$[2.87]$} \\
\hline \multicolumn{5}{|l|}{ Medio } \\
\hline \multirow[t]{2}{*}{ Matemática } & 362 & 423 & 405 & 380 \\
\hline & {$[2.96]$} & {$[5.26]$} & {$[9.52]$} & [ 2.90] \\
\hline \multirow[t]{2}{*}{ Lectura } & 361 & 438 & 421 & 384 \\
\hline & {$[3.99]$} & [5.39] & [8.98] & {$[3.58]$} \\
\hline \multirow[t]{2}{*}{ Ciencia } & 376 & 442 & 422 & 395 \\
\hline & {$[3.01]$} & {$[5.35]$} & {$[8.66]$} & {$[3.02]$} \\
\hline Bajo & & & & \\
\hline Matemática & 341 & 383 & 373 & 348 \\
\hline & [2.99] & {$[6.91]$} & {$[8.97]$} & {$[2.76]$} \\
\hline Lectura & 339 & 390 & 383 & 348 \\
\hline & {$[3.14]$} & [11.64] & {$[13.06]$} & {$[2.85]$} \\
\hline Ciencia & 350 & 399 & 387 & 358 \\
\hline & {$[2.87]$} & {$[8.53]$} & {$[10.71]$} & {$[2.72]$} \\
\hline
\end{tabular}


Table A3.1 (cont.): Puntajes promedios de las pruebas PISA según circunstancias del alumno.

\begin{tabular}{|c|c|c|c|c|}
\hline \multirow{2}{*}{$\begin{array}{c}\text { Pomedio puntajes } \\
\text { pruebas PISA }\end{array}$} & \multicolumn{3}{|c|}{ Tipo de establecimiento } & \multirow{2}{*}{ Total } \\
\hline & Público & Privado subvencionado & Privado independiente & \\
\hline \multicolumn{5}{|c|}{ Según clima educativo del colegio } \\
\hline \multicolumn{5}{|l|}{ Alto } \\
\hline \multirow[t]{2}{*}{ Matemática } & 410 & 449 & 461 & 442 \\
\hline & {$[10.48]$} & {$[4.44]$} & {$[12.16]$} & {$[4.92]$} \\
\hline \multirow[t]{2}{*}{ Lectura } & 423 & 468 & 473 & 457 \\
\hline & {$[12.73]$} & {$[7.17]$} & {$[12.79]$} & {$[5.54]$} \\
\hline \multirow[t]{2}{*}{ Ciencia } & 434 & 471 & 473 & 461 \\
\hline & {$[10.03]$} & {$[4.19]$} & {$[12.16]$} & {$[4.48]$} \\
\hline \multicolumn{5}{|l|}{ Medio } \\
\hline \multirow[t]{2}{*}{ Matemática } & 357 & 406 & 386 & 366 \\
\hline & {$[3.32]$} & {$[8.77]$} & {$[9.44]$} & {$[3.28]$} \\
\hline \multirow[t]{2}{*}{ Lectura } & 353 & 416 & 394 & 365 \\
\hline & {$[3.81]$} & {$[10.37]$} & {$[11.42]$} & {$[3.61]$} \\
\hline \multirow[t]{2}{*}{ Ciencia } & 367 & 422 & 404 & 377 \\
\hline & [3.32] & [9.41] & {$[10.60]$} & {$[3.36]$} \\
\hline \multicolumn{5}{|l|}{ Bajo } \\
\hline Matemática & 320 & 385 & s.d. & 332 \\
\hline & [12.93] & [7.46] & & [12.11] \\
\hline Lectura & 319 & 369 & s.d. & 330 \\
\hline & [13.18] & {$[26.20]$} & & {$[12.58]$} \\
\hline Ciencia & 329 & 412 & s.d. & 343 \\
\hline & [11.66] & {$[15.11]$} & & [12.19] \\
\hline Según calidad de lc & $s$ recursos & educativos del colegio & & \\
\hline Alto & & & & \\
\hline Matemática & 387 & 451 & 463 & 418 \\
\hline & {$[4.41]$} & {$[6.76]$} & {$[14.25]$} & {$[4.04]$} \\
\hline Lectura & 388 & 467 & 475 & 425 \\
\hline & [5.78] & [6.66] & [14.89] & {$[4.58]$} \\
\hline Ciencia & 399 & 465 & 479 & 430 \\
\hline & {$[5.41]$} & [7.13] & {$[14.02]$} & {$[4.46]$} \\
\hline Medio & & & & \\
\hline Matemática & 368 & 433 & 434 & 392 \\
\hline & {$[5.42]$} & [6.60] & {$[17.43]$} & {$[5.28]$} \\
\hline Lectura & 370 & 450 & 442 & 399 \\
\hline & [7.30] & [6.94] & {$[18.63]$} & {$[6.46]$} \\
\hline Ciencia & 381 & 454 & 442 & 407 \\
\hline & [6.08] & [6.27] & {$[16.73]$} & {$[5.55]$} \\
\hline Bajo & & & & \\
\hline Matemática & 341 & 395 & 393 & 354 \\
\hline & [5.55] & [9.43] & {$[13.38]$} & {$[4.78]$} \\
\hline Lectura & 334 & 402 & 408 & 350 \\
\hline & [6.00] & {$[13.83]$} & {$[12.14]$} & {$[5.19]$} \\
\hline Ciencia & 350 & 419 & 420 & 367 \\
\hline & [5.19] & [11.45] & {$[11.49]$} & {$[4.42]$} \\
\hline Según calidad de lc & $s$ profesor & es del colegio & & \\
\hline Alto & & & & \\
\hline Matemática & 400 & 442 & 453 & 414 \\
\hline & [9.40] & {$[9.07]$} & {$[55.61]$} & {$[7.60]$} \\
\hline Lectura & 397 & 469 & 478 & 421 \\
\hline & [11.03] & [12.11] & {$[58.77]$} & {$[9.56]$} \\
\hline Ciencia & 411 & 465 & 471 & 429 \\
\hline & {$[12.19]$} & {$[10.03]$} & {$[46.32]$} & {$[9.27]$} \\
\hline Medio & & & & \\
\hline Matemática & 380 & 425 & 453 & 409 \\
\hline & [8.30] & [11.41] & [30.33] & {$[6.32]$} \\
\hline Lectura & 373 & 446 & 466 & 414 \\
\hline & [10.09] & [11.49] & {$[35.21]$} & {$[8.04]$} \\
\hline Ciencia & 390 & 453 & 465 & 425 \\
\hline & [8.49] & {$[10.54]$} & {$[32.44]$} & {$[6.88]$} \\
\hline Bajo & & & & \\
\hline Matemática & 354 & 436 & 438 & 381 \\
\hline & {$[5.57]$} & {$[9.12]$} & {$[28.81]$} & {$[6.36]$} \\
\hline Lectura & 354 & 451 & 446 & 386 \\
\hline & {$[8.01]$} & [8.87] & {$[22.48]$} & {$[7.51]$} \\
\hline Ciencia & 363 & 454 & 449 & 393 \\
\hline & [6.09] & [9.34] & {$[20.90]$} & {$[6.45]$} \\
\hline
\end{tabular}




\section{Gráficos.}

Gráfico A3.1: Funciones de distribución acumulada según tipo de establecimiento.
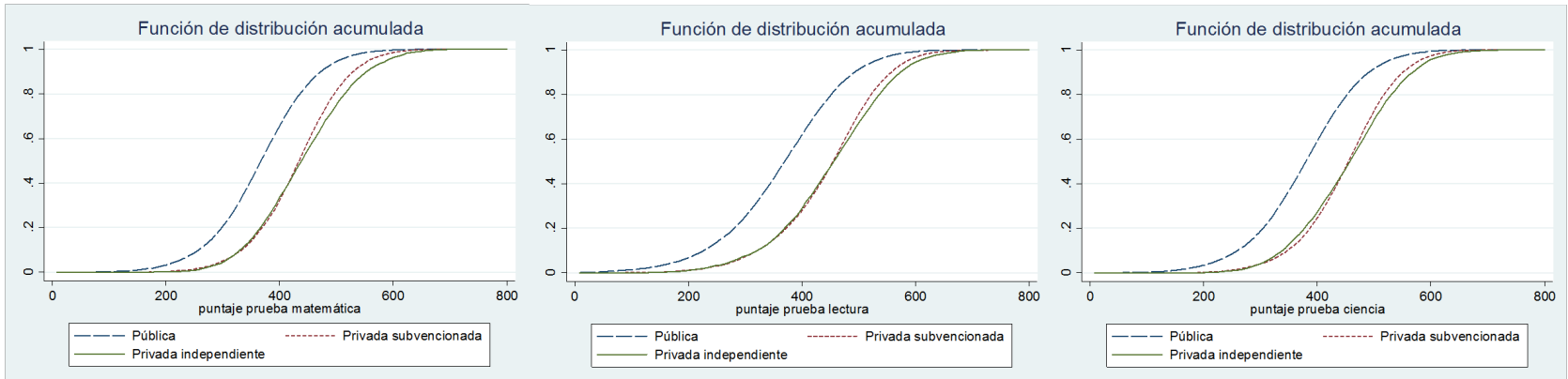

Fuente: Elaboración propia en base a datos PISA de los años 2006, 2009 y 2012.

Gráfico A3.2: Funciones de distribución acumulada según tipo de establecimiento y esfuerzo.

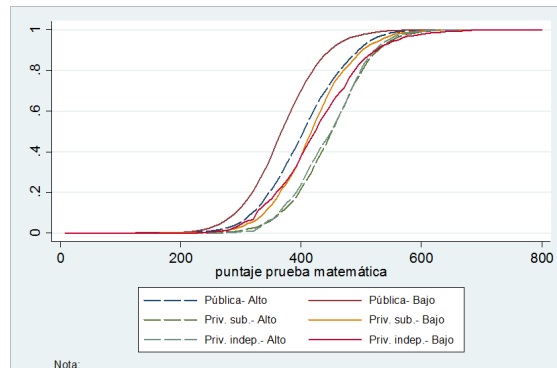

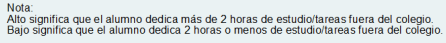

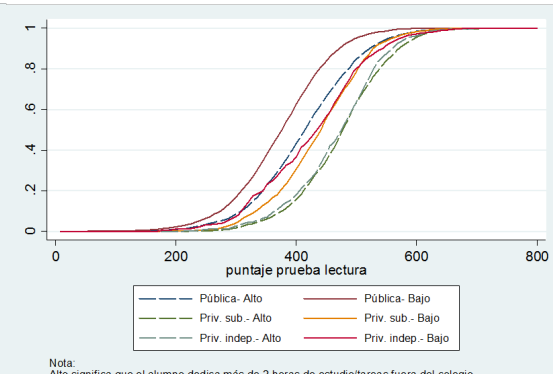

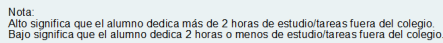

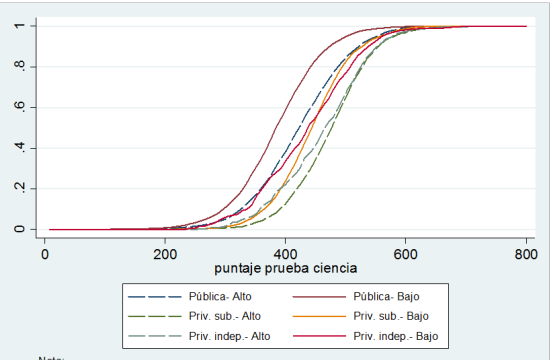

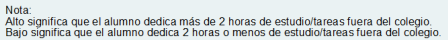

Fuente: Elaboración propia en base a datos PISA del año 2012.

Gráfico A3.3: Funciones de distribución acumulada según tipo de establecimiento y esfuerzO.
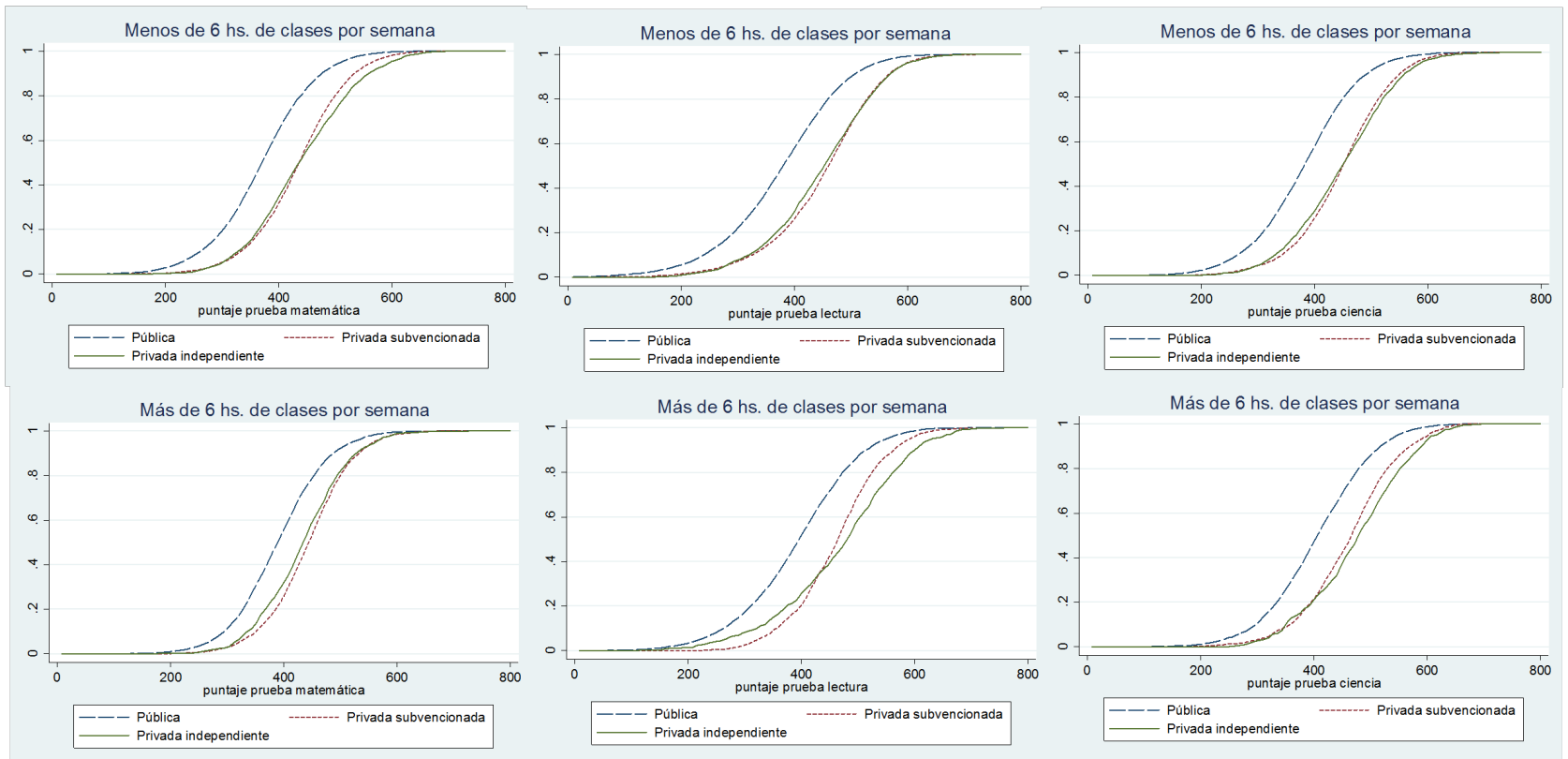

Fuente: Elaboración propia en base a datos PISA de los años 2006, 2009 y 2012. 
Gráfico A3.4: Funciones de distribución acumulada según tipo de establecimiento, esfuerzo y educación de los padres.
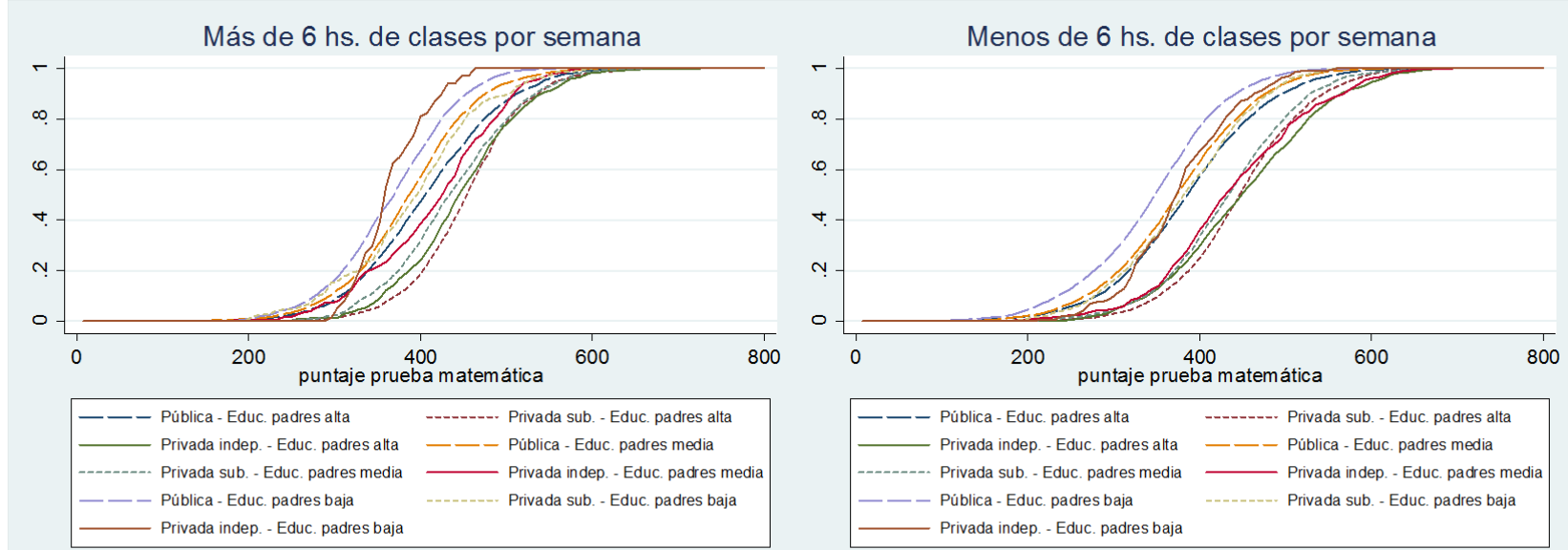

Más de 6 hs. de clases por semana
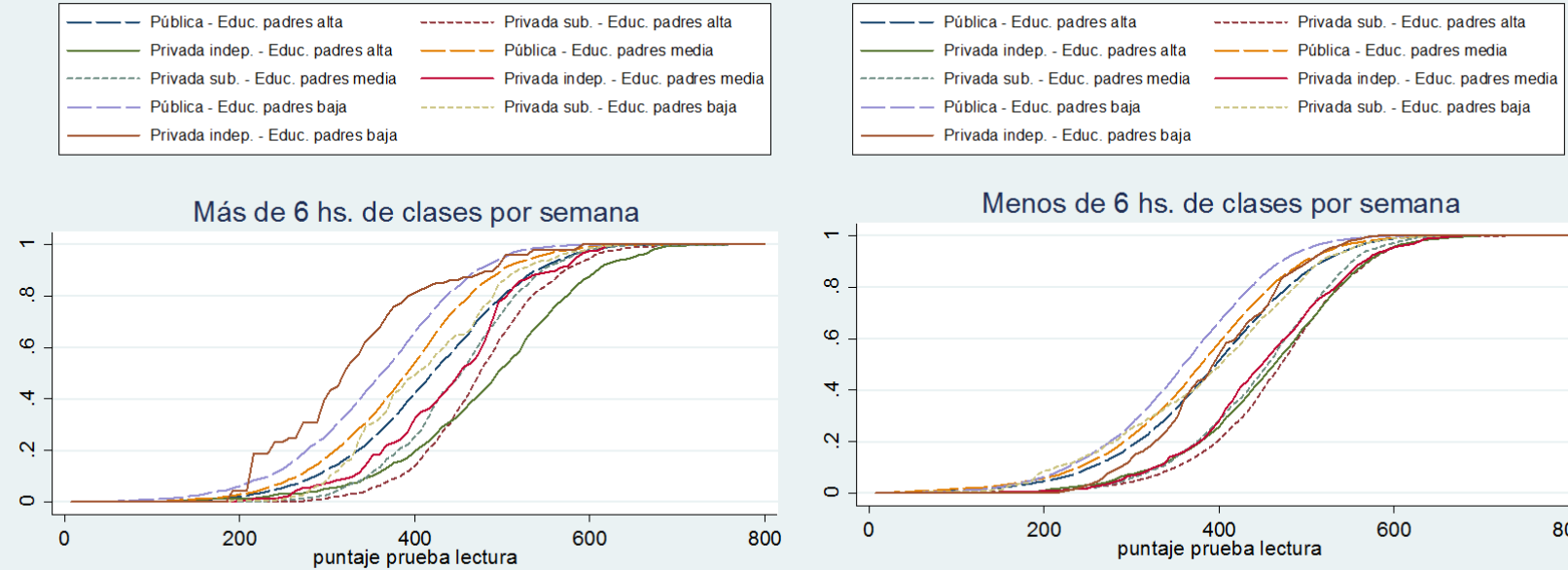

Privada indep. - Educ. padres baja

Menos de 6 hs. de clases por semana

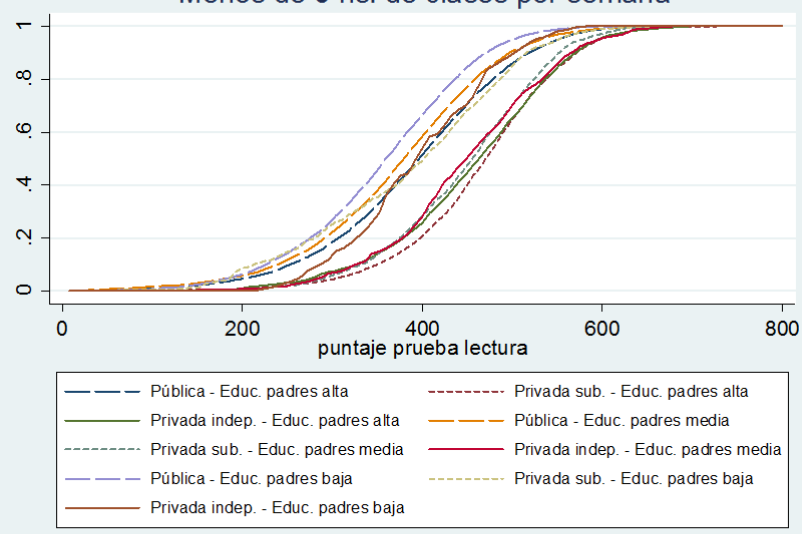

Más de 6 hs. de clases por semana
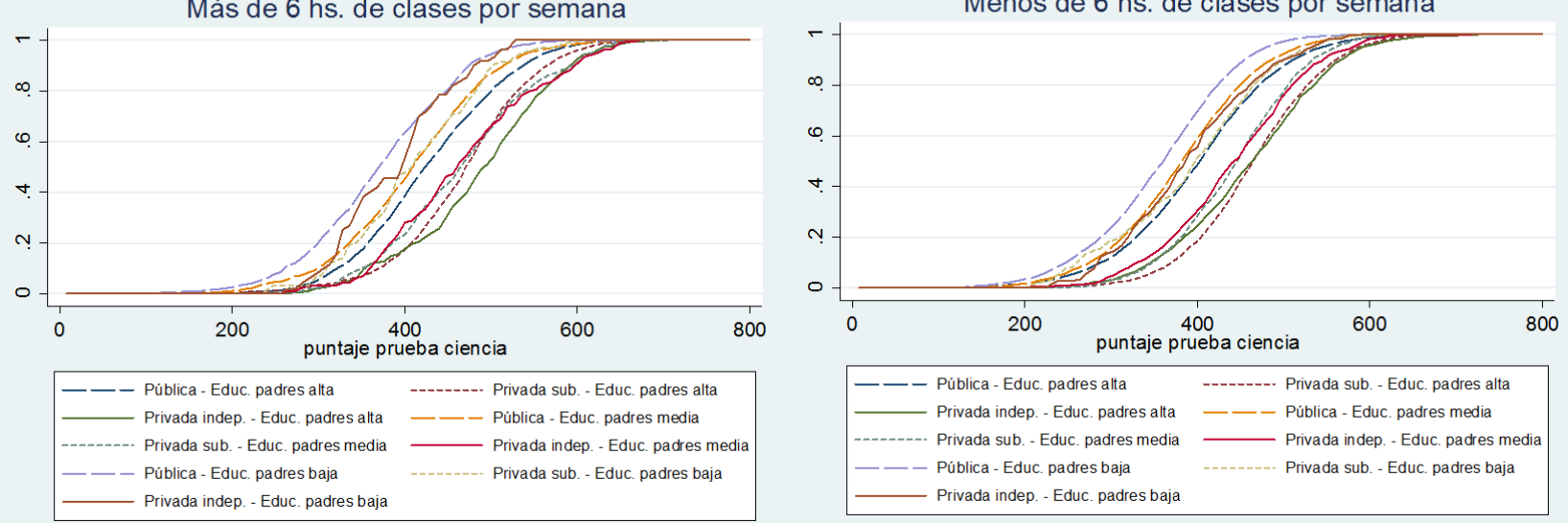

\begin{tabular}{|c|c|}
\hline 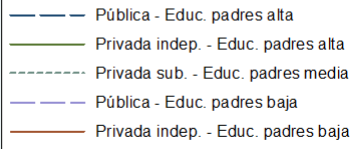 & $\begin{array}{l}------- \text { Privada sub. - Educ. padres alta } \\
-\_- \text {Püblica - Educ. padres media } \\
- \text { Privada indep. - Educ. padres media } \\
- \text { Privada sub. - Educ. padres baja }\end{array}$ \\
\hline
\end{tabular}

Fuente: Elaboración propia en base a datos PISA de los años 2006, 2009 y 2012. 
Resultados de test de funciones de distribuciones acumuladas.

Tabla A3.2: Test de igualdad y de dominancia estocástica de distribuciones de puntajes de lectura según tipo de establecimiento, esfuerzo y nivel educativo de los padres.

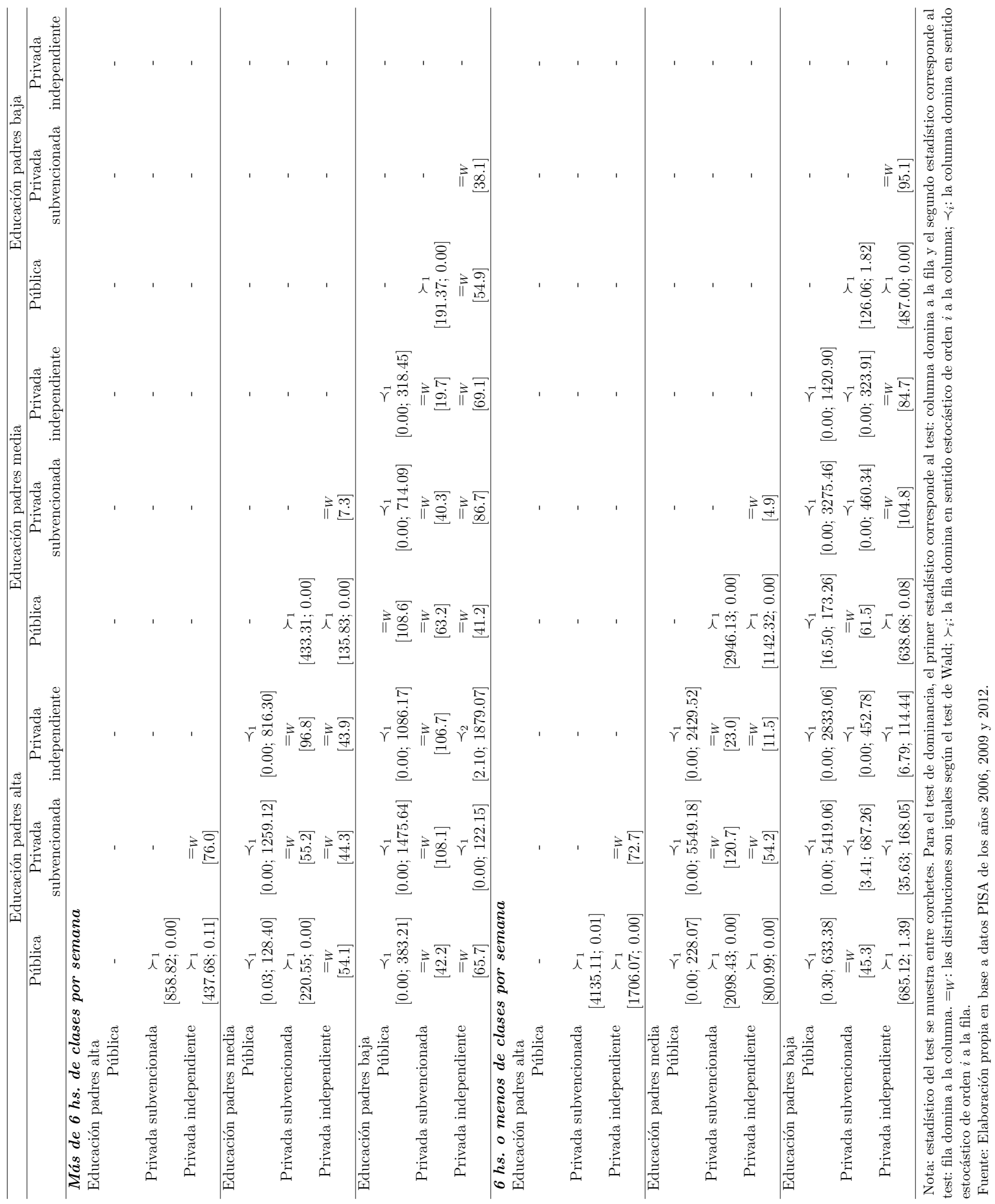


Tabla A3.3: Test de igualdad y de dominancia estocástica de distribuciones de puntajes de ciencia según tipo de establecimiento, esfuerzo y nivel educativo de los padres.

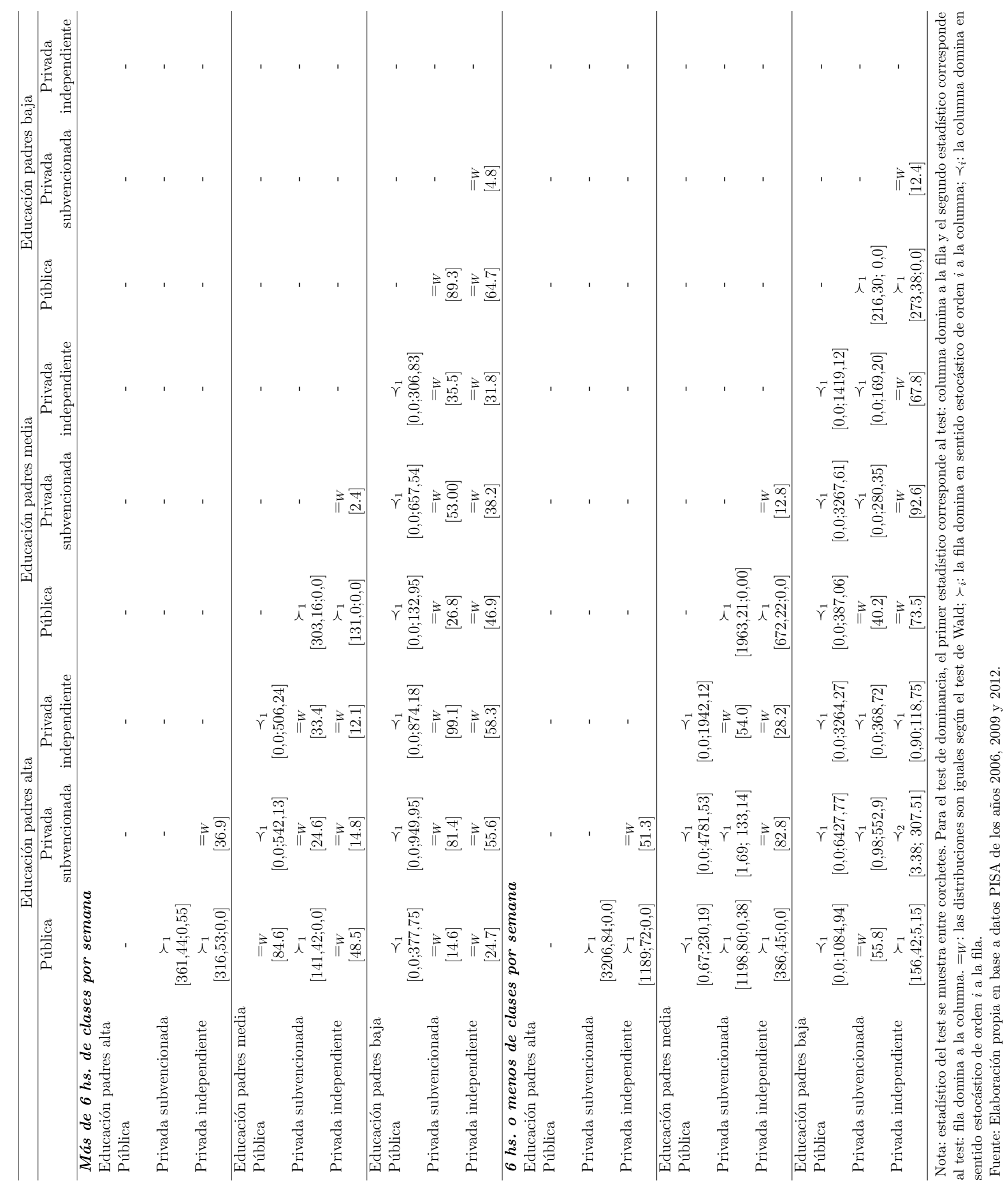


Tabla A3.4: Test de igualdad y de dominancia estocástica de distribuciones de puntajes de lectura según tipo de establecimiento, esfuerzo y nivel socioeconómico familiar.

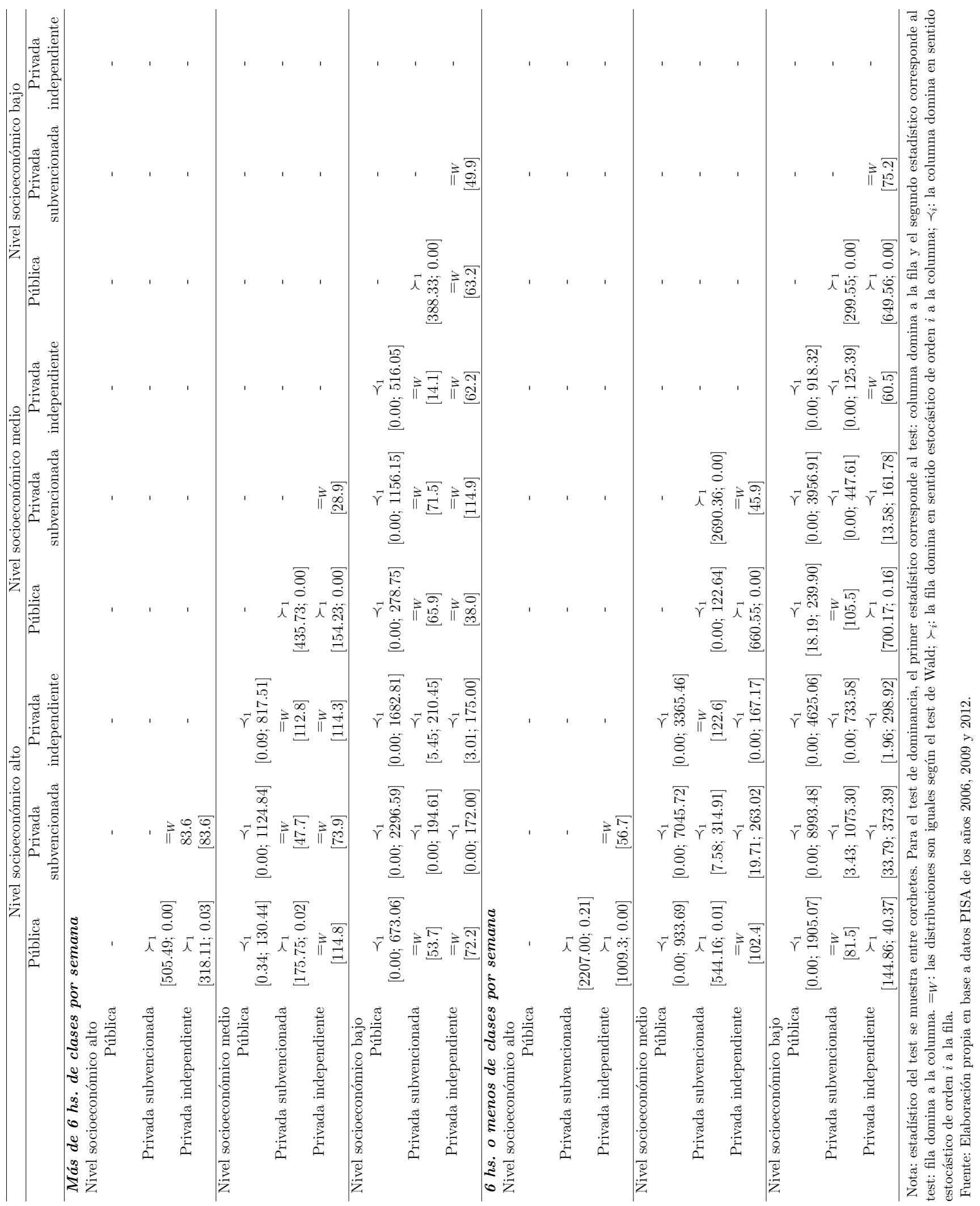


Tabla A3.5: Test de igualdad y de dominancia estocástica de distribuciones de puntajes de ciencia según tipo de establecimiento, esfuerzo y nivel socioeconómico familiar.

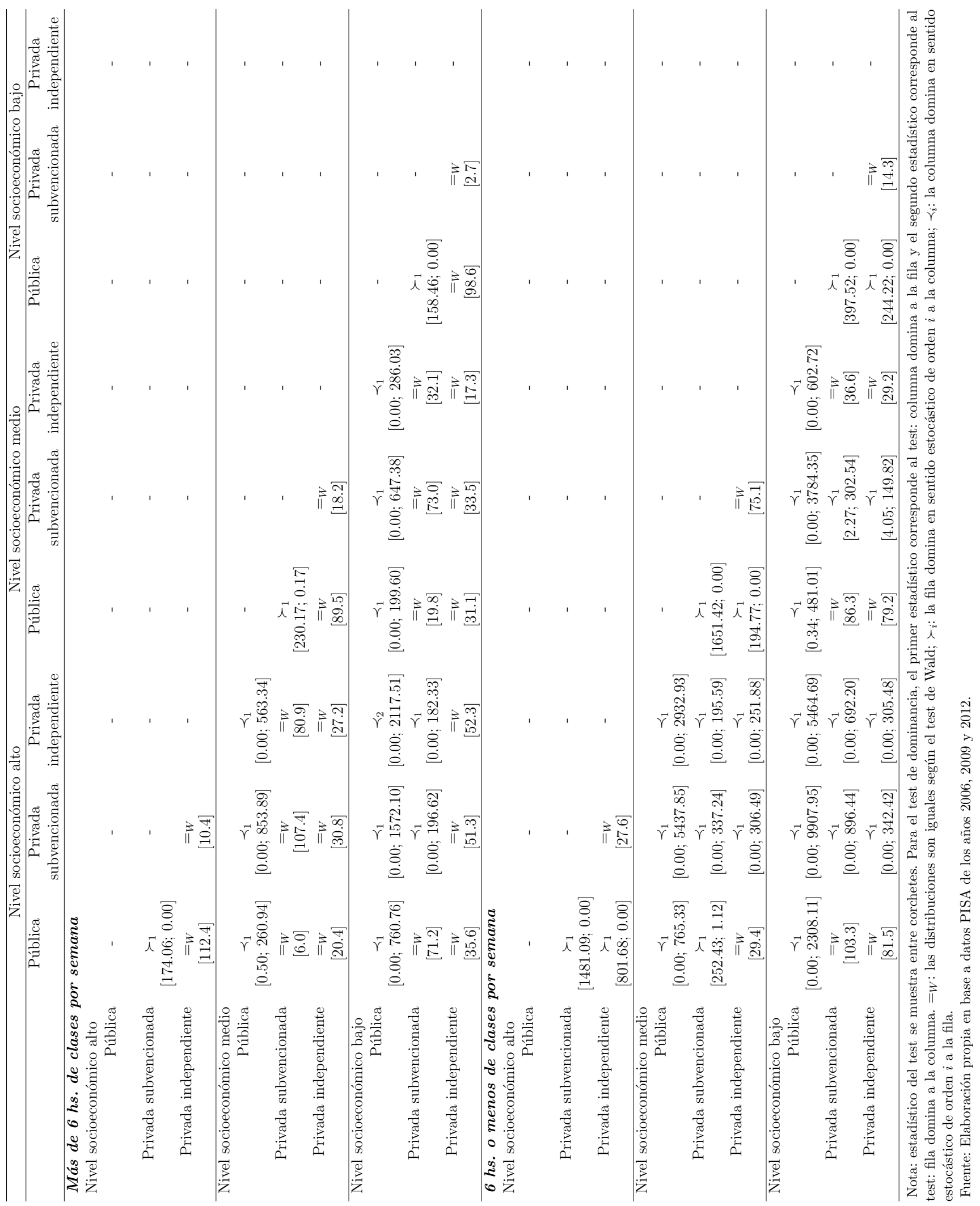


Tabla A3.6: Test de igualdad y de dominancia estocástica de distribuciones de puntajes de lectura según tipo de establecimiento, esfuerzo y clima educativo.

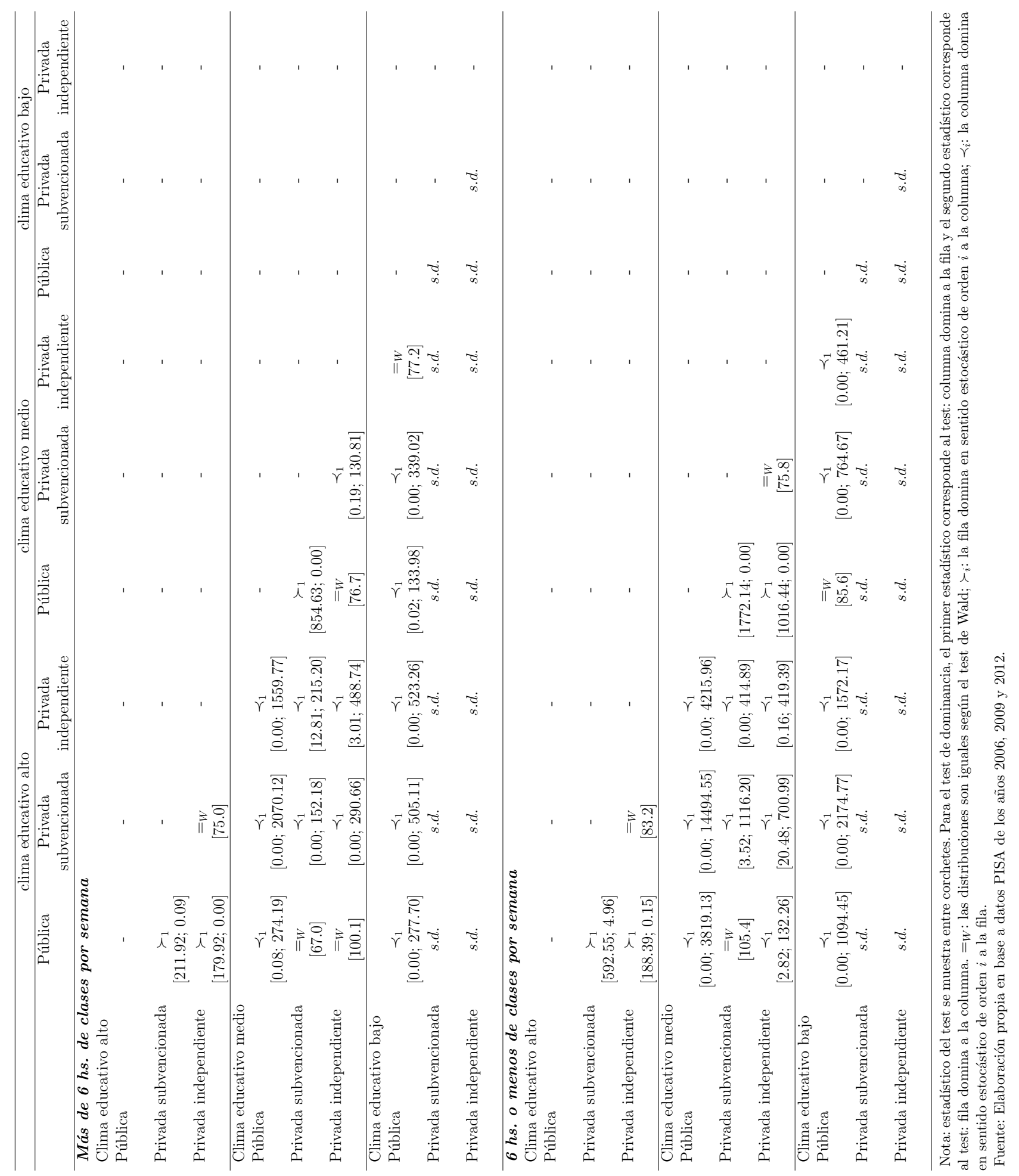


Tabla A3.7: Test de igualdad y de dominancia estocástica de distribuciones de puntajes de ciencia según tipo de establecimiento, esfuerzo y clima educativo.

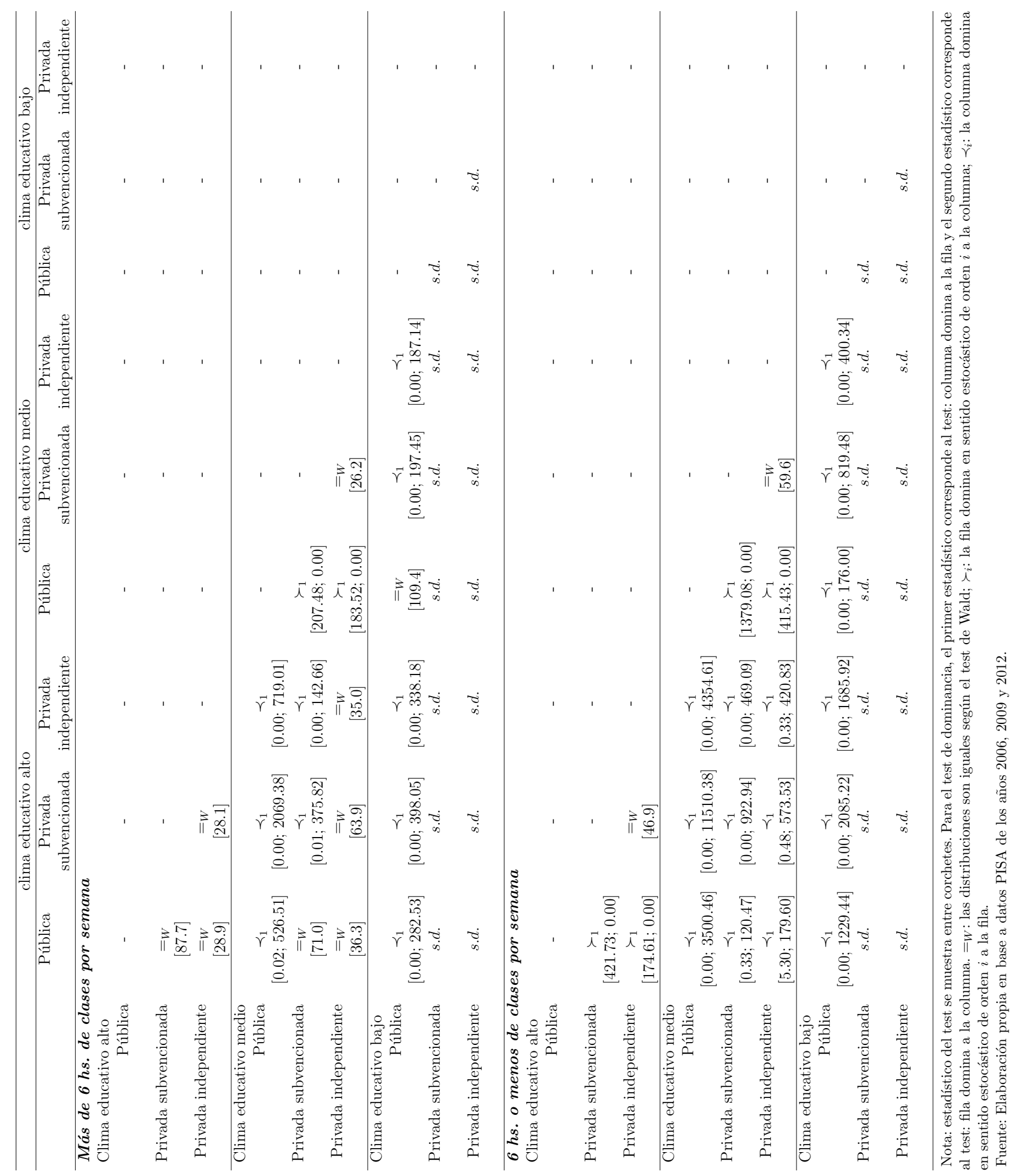


Tabla A3.8: Test de igualdad y de dominancia estocástica de distribuciones de puntajes de lectura según tipo de establecimiento, esfuerzo y calidad de recursos educativos.

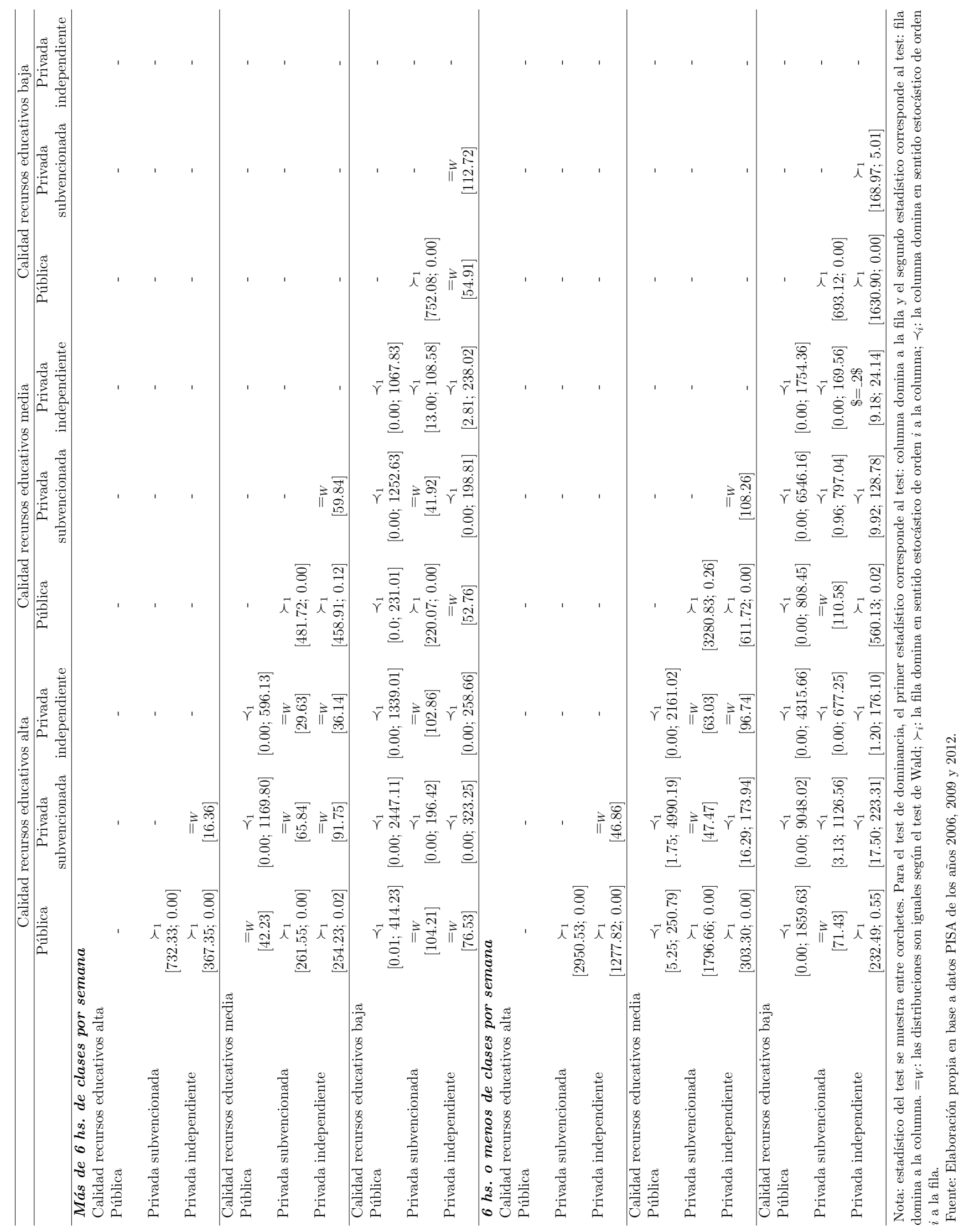


Tabla A3.9: Test de igualdad y de dominancia estocástica de distribuciones de puntajes de ciencia según tipo de establecimiento, esfuerzo y calidad de recursos educativos.

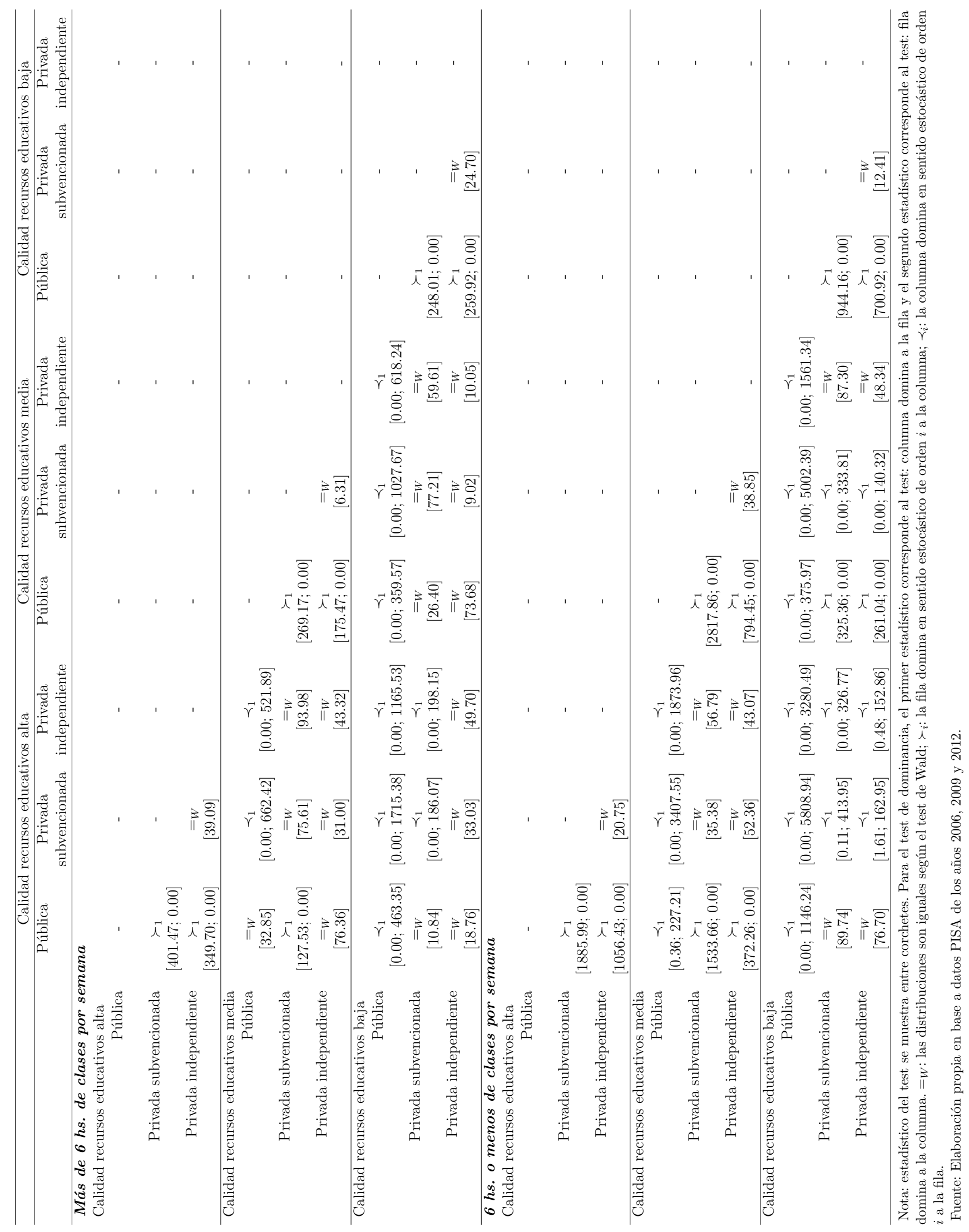


Tabla A3.10: Test de igualdad y de dominancia estocástica de distribuciones de puntajes de lectura según tipo de establecimiento, esfuerzo y calidad de los profesores del colegio.

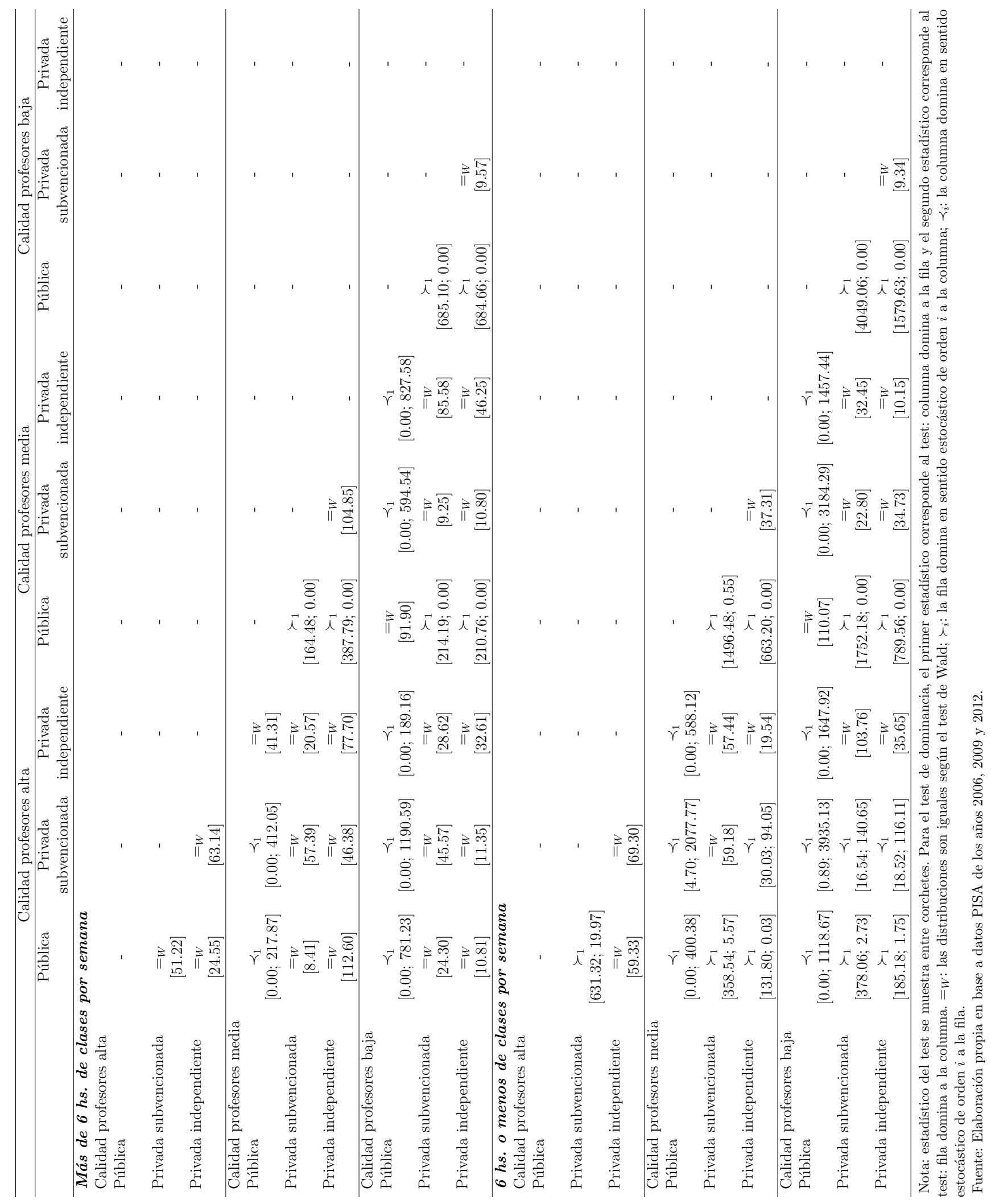


Tabla A3.11: Test de igualdad y de dominancia estocástica de distribuciones de puntajes de ciencia según tipo de establecimiento, esfuerzo y calidad de los profesores del colegio.

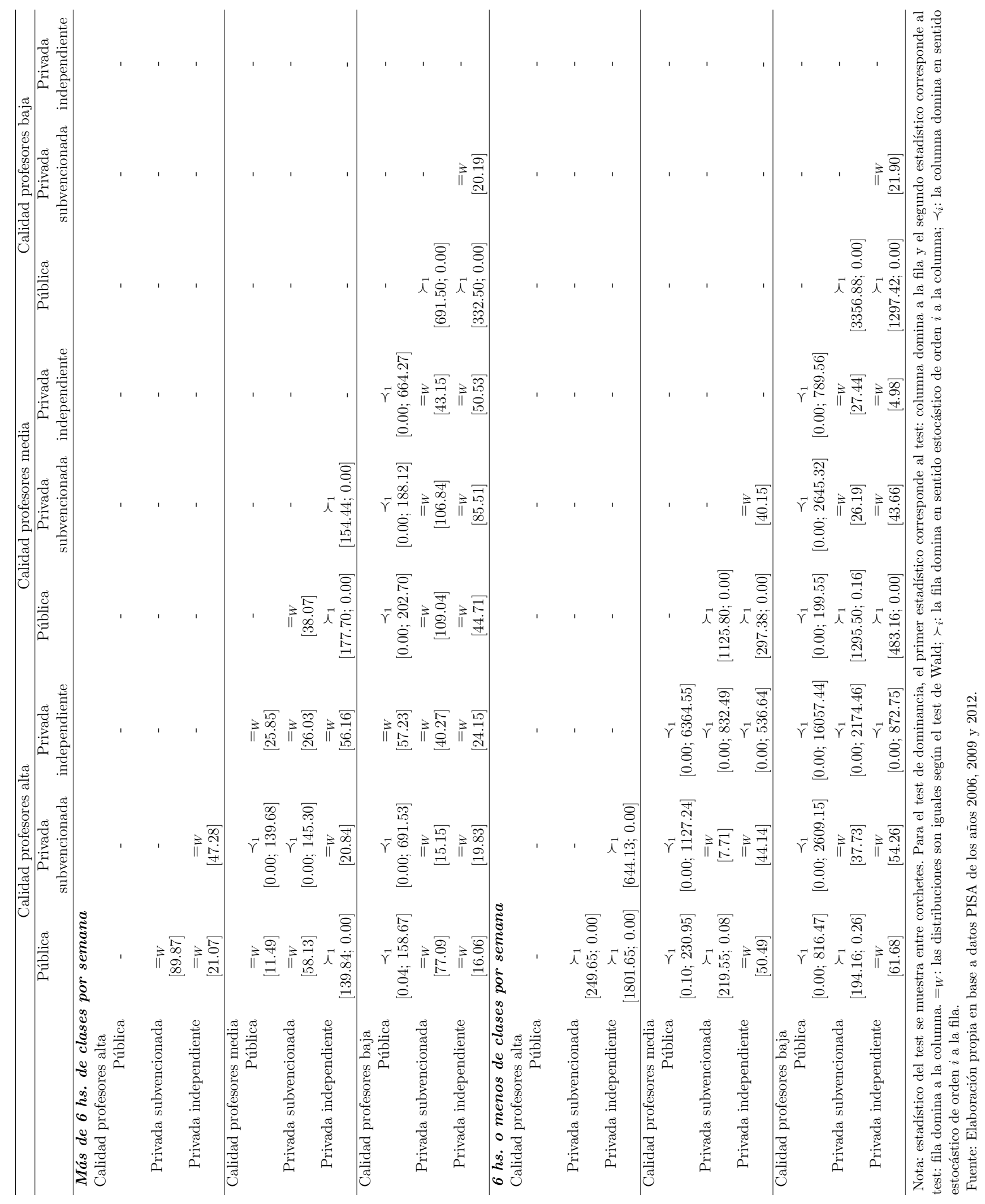

